Europäische Hochschulschriften

Rechtswissenschaft

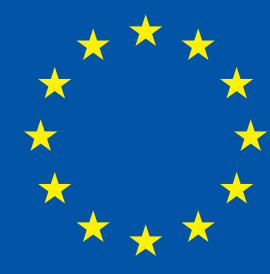

Xiaopeng Zhao

\title{
Die rechtliche Regulierung der Menschenfleischsuche im Internet
}

Eine rechtsvergleichende Untersuchung zwischen Deutschland und China 


\section{PETER LANG · Academic Research}

\section{II / 5754}

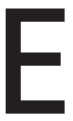

rstmalig thematisiert das Buch das in China weit verbreitete Phänomen Ren-rou-sou-suo (Menschenfleischsuche) auch in Deutschland. Als Hexenjagd im Internet ist dieses weltweite Phänomen auch in Deutschland in den nächsten Jahren zu erwarten. Aufgrund potenzieller Persönlichkeitsrechtsverletzungen und Datenschutzprobleme ist es hoch regulierungsbedürftig. Der Autor analysiert die Hintergründe der Suche in beiden Ländern unter technischen, kulturellen, sozialen, politischen und psychologischen Aspekten. Er untersucht und beantwortet rechtsvergleichend und detailliert alle diesbezüglichen Rechtsfragen einschließlich Providerhaftung, Anonymität und Pseudonymität, Auskunftsanspruch auf Anmeldedaten von Nutzern, Notice and take down sowie Störerhaftung.

Xiaopeng Zhao ist promovierter Jurist in Göttingen und Volljurist in China. Derzeit arbeitet er als Legal Consultant in München. Seine Arbeitsschwerpunkte sind IP-Recht und Internetrecht. 
Die rechtliche Regulierung der

Menschenfleischsuche im Internet 


\section{Europäische Hochschulschriften}

European University Studies

Publications Universitaires Européennes

Reihe II Rechtswissenschaft

Series II Law

Série II Droit

Band/Volume 5754 
Xiaopeng Zhao

\section{Die rechtliche Regulierung der Menschenfleischsuche im Internet}

Eine rechtsvergleichende Untersuchung zwischen Deutschland und China

\section{PETER LANG}


Bibliografische Information der Deutschen Nationalbibliothek Die Deutsche Nationalbibliothek verzeichnet diese Publikation in der Deutschen Nationalbibliografie; detaillierte bibliografische Daten sind im Internet über http://dnb.d-nb.de abrufbar.

Zugl.: Göttingen, Univ., Diss., 2015

Gedruckt auf alterungsbeständigem, säurefreiem Papier.

D 7

ISSN $0531-7312$

ISBN 978-3-631-66365-3 (Print)

E-ISBN 978-3-653-05811-6 (E-Book)

DOI 10.3726/978-3-653-05811-6

\section{PETER LANG

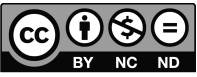

Open Access: Dieses Werk ist lizensiert unter der Creative Commons Lizenz Namensnennung - Nicht kommerziell - Keine Bearbeitungen 4.0 International (CC BY-NC-ND 4.0). Den vollständigen Lizenztext finden Sie unter: https://creativecommons.org/licenses/by-nc-nd/4.0/deed.de

(C) Xiaopeng Zhao, 2015

Peter Lang $\mathrm{GmbH}$

Internationaler Verlag der Wissenschaften

Berlin

Peter Lang - Berlin · Bern · Bruxelles · New York · Oxford · Warszawa - Wien Diese Publikation wurde begutachtet.

www.peterlang.com 


\section{Inhaltsverzeichnis}

Abkürzungsverzeichnis

XIX

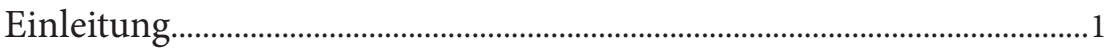

$\$ 1$. Menschenfleischsuche als ein soziales Phänomen .............................................

I. Die originale Bedeutung der Menschenfleischsuche in China .................3

II. Die geänderte Bedeutung der Menschenfleischsuche in China ..............4

III. Die in dieser Arbeit zu diskutierende Menschenfleischsuche ..................5

1. Die Motivationen der Menschenfleischsuche und die Beispiele in China .5

a) Um Korruption oder anderes rechtswidriges Verhalten von Beamten oder anderen Prominenten aufzudecken........................ 5

b) Um rechtswidriges Verhalten normaler Bürger aufzudecken......................................................................6

c) Um gegen unmoralisches Verhalten zu kämpfen...........................6

d) Um die Person in schwieriger Situation zu helfen ........................8

e) Um Rache auszuüben....................................................................8

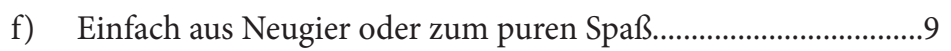

g) Zum Zweck der Werbung..............................................................9

2. Der Vorgang der in dieser Arbeit zu diskutierende Menschenfleischsuche ......................................................................... 11

IV. Der Unterschied zwischen Menschenfleischsuche und anderen ähnlichen Phänomen ............................................................. 12

1. Der Unterschied mit Suchmeldung ....................................................... 12

2. Der Unterschied mit der Öffentlichkeitsfahndung

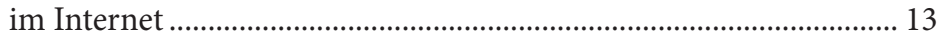

3. Der Unterschied mit dem Klatsch im Alltagsleben............................. 13

4. Der Unterschied mit der Personensuchmaschine ............................... 14

V. Die Hintergründe der Entstehung und schnellen Entwicklung der Menschenfleischsuche in China................................... 15

1. Technischer Hintergrund....................................................................... 15

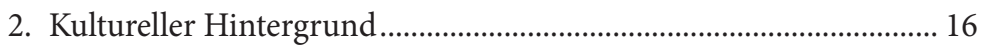


a) Schwache Gewichtung vom Schutz der Privatsphäre................... 16

b) Schwaches Rechtsbewusstsein des Volkes ..................................... 18

3. Politische und soziale Hintergründe ........................................................ 19

a) Die Explosion der gebannten Meinungsfreiheit im Internet

b) Das Misstrauen der Bürger gegen die Hauptmedien

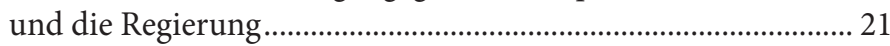

4. Psychologischer Hintergrund.................................................................. 22

VI. Die Bewertung der Menschenfleischsuche in China .............................. 23

1. Die negativen Seiten der Menschenfleischsuche .................................. 23

a) Menschenfleischsuche hat den Charakter

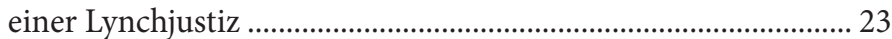

b) Irrationalität und Einseitigkeit ....................................................... 24

c) Die Gefahr falscher Verdächtigung ................................................. 25

d) Die Gefahr der Informant-Kultur ................................................. 25

2. Die positiven Seiten der Menschenfleischsuche .................................. 25

a) Um Korruption oder anderes rechtswidriges Verhalten von Beamten aufzudecken ................................................................ 25

b) Um rechtswidriges Verhalten, insbesondere das Verbrechen der normalen Bürger aufzudecken ............................ 26

c) Zur Entwicklung der demokratischen Kultur in China............... 26

d) Die Funktion als soziales Sicherheitsventil in China.................... 26

3. Die Bewertung der Menschenfleischsuche .......................................... 27

VII. Menschenfleischsuche als ein soziales Phänomen weltweit................... 28

1. Ein paar Beispiele der Menschenfleischsuche weltweit ...................... 28

2. Menschenfleischsuche und ähnliches Phänomen in Deutschland

a) Private Facebook-Jagd nach einem unbekannten Sextäter-Menschenfleischsuche in Deutschland ......................... 30

b) Aufruf zum Lynchmord gegen einen 17-jährigen Verdächtigten auf Facebook - Menschenfleischsuche ähnliches Phänomen

c) Die anderen Menschenfleischsuche ähnlichen Ereignisse in Deutschland. 
$\$ 2$ Menschenfleischsuche als ein rechtliches Phänomen..................................33

I. Die Rechtsprobleme während Menschenfleischsuche ............................ 33

1. Die Rechtsprobleme allgemein............................................................ 33

2. Die Rechtsprobleme bezüglich der Veranlasser.................................. 33

3. Die Rechtsprobleme bezüglich der Informationssammler................ 33

4. Die Rechtsprobleme bezüglich der Informationsberichter ............... 34

5. Die Rechtsprobleme bezüglich der ICP und ISP ................................ 34

6. Die Rechtsprobleme bezüglich der Zielperson ..................................... 35

7. Die Rechtsprobleme bezüglich der Kommentatoren ........................... 35

II. Die Wichtigkeit der wissenschaftlichen Diskussion über

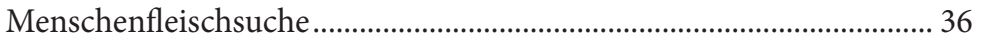

1. Geographisch unkontrollierbare Verbreitung der

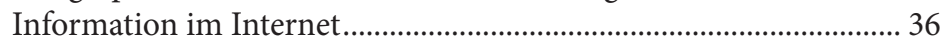

2. Zeitlich unkontrollierbare Verbreitung der Information

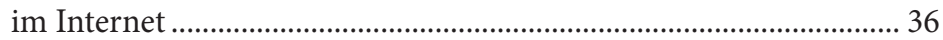

3. Unkontrollierbare Vermehrung der Information im Internet.......... 37

4. Bildung persönlicher Profile und die Prangerwirkung durch Menschenfleischsuche ....................................................................... 37

5. Menschenfleischsuche bietet ein typisches Beispiel der Rechtsverletzung durch den Internetnutzer........................................ 38

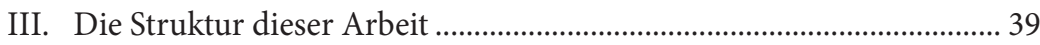

1. Kapitel. Die zu schützenden Rechte der Zielperson während der Menschenfleischsuche

$\S 3$ Der Schutz des Rechts am eigenen Bild.......................................................43

I Der Schutz des Rechts am eigenen Bild in Deutschland .......................... 43

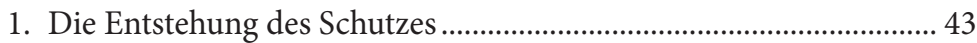

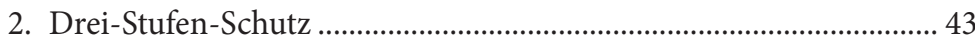

3. Das Recht am eigenen Bild als ein besonderes Persönlichkeitsrecht................................................................................. 44

4. Ansprüche wegen Verletzung des Rechts am eigenen Bild ............... 45

II Der Schutz des Rechts am eigenen Bild in China..................................... 45 
1. Die Diskussion über das gewerbliche Ziel im $₫ 100$

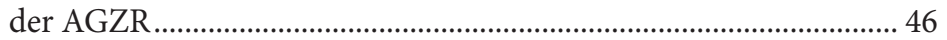

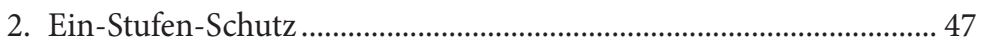

3. Schutz des Rechts am eigenen Bild durch den Schutz der

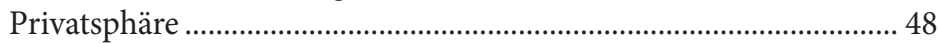

$\$ 4$ Der Schutz des Rechts auf informationelle Selbstbestimmung ....................49

I Der Schutz des Rechts auf informationelle Selbstbestimmung in Deutschland

1. Das Recht auf informationelle Selbstbestimmung und BDSG

2. Die Anwendbarkeit des BDSG auf die Fälle in dieser

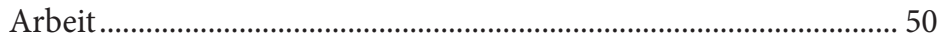

a) Die Eingrenzung personenbezogener Daten .............................. 50

b) Die Anwendbarkeit des BDSG auf die von den Internetnutzern begangenen Rechtsverletzungen...................... 51

c) Die Anwendbarkeit des BDSG auf der von den ICP begangenen Rechtsverletzung. 52

aa) Erhebung, Verarbeitung und Nutzung personenbezogener Daten.............................................................. 53

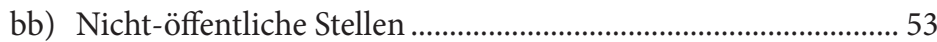

cc) Einsatz von Datenverarbeitungsanlagen ...................................... 53

dd) Kein persönlicher oder familiärer Zweck.................................... 54

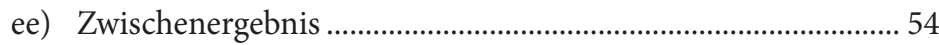

ff) Die Anwendbarkeit des $₫ 28$ BDSG auf der vom ICP begangenen Rechtsverletzung während Menschenfleischsuche

gg) Die Anwendbarkeit des $₫ 29$ BDSG auf die vom ICP begangene Rechtsverletzung während

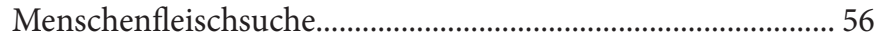

d) Die Anwendbarkeit des BDSG auf das Verhalten des ISP während Menschenfleischsuche .................................... 57

3. Die Anwendbarkeit der anderen Gesetze ………………………...... 58

II Der persönliche Datenschutz in China..................................................... 59

1. Die Geschichte und der gegenwärtige Stand der Legislative vom persönlichen Datenschutz in China 
2. Die Anwendbarkeit der „Informationsschutz-Entscheidung“ auf Menschenfleischsuche.

3. Die Eingrenzung der zu schützenden persönlichen Daten. 61

$\$ 5$ Der Schutz des Rechts auf Privatsphäre. 63

I. Der Schutz des Rechts auf Privatsphäre in Deutschland 63

1. Schutz der Privatsphäre durch Schutz allgemeiner Persönlichkeitsrechte 63

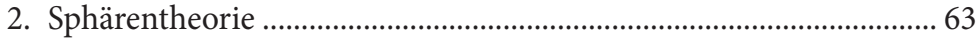

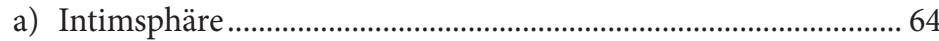

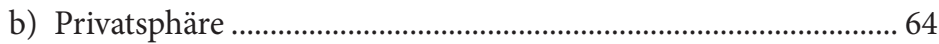

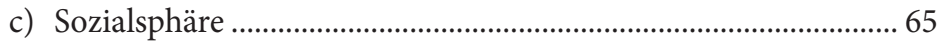

II. Der Schutz des Rechts auf Privatsphäre in China .................................. 66

1. Die Rechtsentwicklung über das Recht auf Privatsphäre

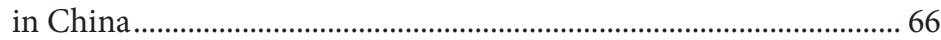

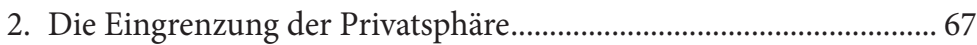

a) Die Unbestimmbarkeit des Inhalts von Privatsphäre.................... 67

b) Die Grenze der Privatsphäre.............................................................. 68

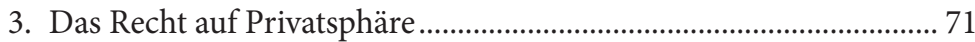

$\$ 6$ Der Schutz des Rechts der persönlichen Ehre ……………………………........73

I. Der Schutz des Rechts der persönlichen Ehre in Deutschland ........................................................................................... 73

II. Der Schutz des Rechts der persönlichen Ehre in China .......................... 74

$\$ 7$ Die Verhältnisse zwischen Schutz am eigenen Bild, Datenschutz, Schutz der Privatsphäre und Ehrenschutz....................................................77

I. Das Verhältnis zwischen dem Schutz am eigenen Bild

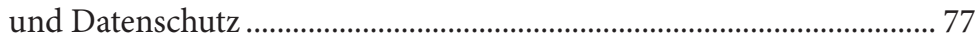

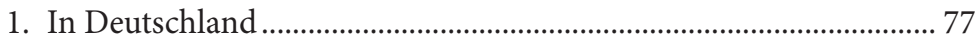

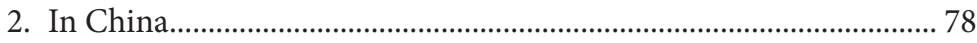

II. Das Verhältnis zwischen Datenschutz und Schutz

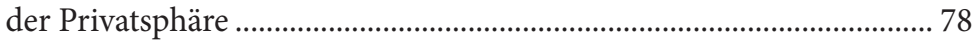

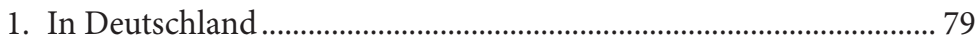

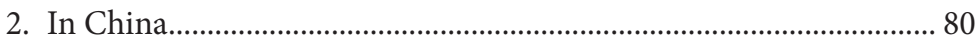


III. Schutz der allgemeinen Persönlichkeitsrechte ......................................... 80

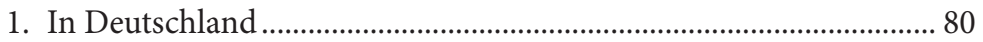

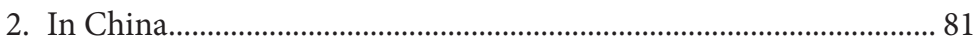

2. Kapitel. Die Identifizierung der Rechtsverletzungen während Menschenfleischsuche

$\$ 8$ Die von den Internetnutzern begangenen

Rechtsverletzungen .85

I. Die von den Informationsberichtern begangenen

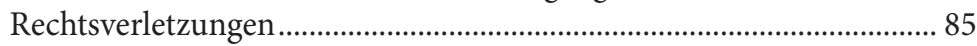

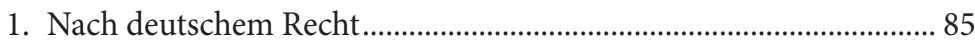

a) Verletzung des Rechts auf Privatsphäre ......................................... 85

b) Verletzung des Rechts am eigenen Bild....................................... 87

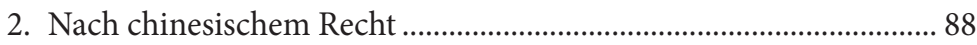

a) Verletzung des Rechts auf Privatsphäre .......................................... 88

b) Verletzung des Rechts am eigenen Bild........................................ 90

II. Die von den Informationssammlern begangenen Rechtsverletzungen................................................................ 90

1. Die Verwendung der ohne Zustimmung veröffentlichten Informationen vom Informationssammler ......................................... 90

a) In Deutschland ............................................................................ 91

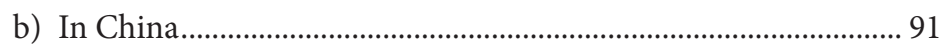

2. Die Verwendung der frei zugänglichen Informationen zum beliebigen Zweck ..................................................................... 92

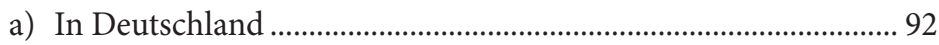

b) In China............................................................................. 92

3. Zwischenergebnis.................................................................................. 93

III. Die von den Veranlassern begangenen Rechtsverletzungen.................. 94

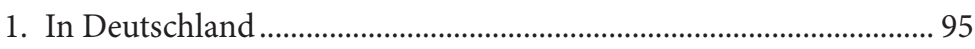

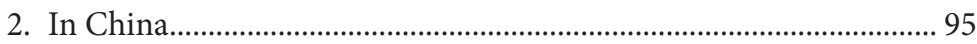

3. Zwischenergebnis.................................................................................. 98

IV. Die von den Kommentator begangenen Rechtsverletzungen................ 98

1. Nach deutschem Recht...................................................................... 98 

a) Werturteil und meinungsbezogene
Tatsachenbehauptung sind grundsätzlich geschützt.
b) Pure Tatsachenbehauptung wird nicht durch Meinungsfreiheit geschützt
c) Unwahre meinungsbezogene Tatsachenbehauptung wird nicht geschützt..... 100
d) Schmähkritik ist gesetzlich verboten ........................................ 100

2. Nach chinesischem Recht ..................................................................... 101

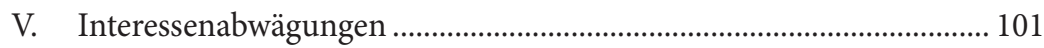

1. Ein Überblick auf die zu diskutierenden Fallgruppen ..................... 102

2. Die Interessenabwägung im Fall der Verletzung des Persönlichkeitsrechts der Prominenten ............................................ 103

a) Nach deutschem Recht .............................................................. 103

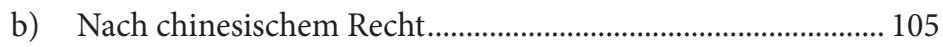

3. Die Interessenabwägung im Fall der Verletzung des Persönlichkeitsrechts der Person aus einem Ereignis ...................... 106

a) Nach deutschem Recht ............................................................ 106

b) Nach chinesischem Recht............................................................ 108

4. Die Interessenabwägung im Fall der Verletzung des Persönlichkeitsrechts der Person mit besonderen Beruf................. 109

a) Nach deutschem Recht .................................................................. 109

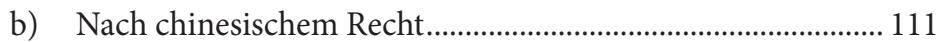

5. Die Interessenabwägung im Fall der Verletzung des Persönlichkeitsrechts des Straftäters.

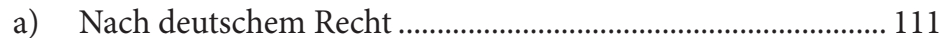

aa) Die Außergewöhnlichkeit.............................................................. 112

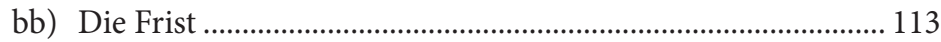

b) Nach chinesischem Recht.......................................................... 114

6. Die Interessenabwägung im Fall der Verletzung des Persönlichkeitsrechts der Parteien des Gerichtsprozesses

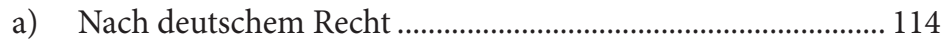

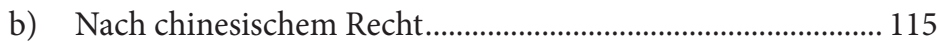

aa) Die Entwicklung der Meinungen in der Entscheidungspraxis 
bb) Die Entwicklung der Rechtsprechungen des Obersten Volksgerichtshofs.

cc) Die Meinungen in der Literatur über die Anonymisierung der zu veröffentlichen Urteile. 118

$\$ 9$ Die von dem ICP begangenen Rechtsverletzungen

I. Nach deutschem Recht.

1. Zueigenmachen fremder Informationen 120

2. Die Daten aus allgemein zugänglichen Quellen 122

3. Das Medienprivileg. 123

a) Rechtmäßiges Subjekt 123

b) Zielsetzung der Datenbearbeitung 124

II. Nach chinesischem Recht 125

$\$ 10$ Die von dem ISP begangenen Rechtsverletzungen

I. Nach deutschem Recht.

1. Die Möglichkeit des ISP als unmittelbarer Verletzer zu haften

a) Der ISP hat keine allgemeine Prüfungspflicht......................... 128

b) Der ISP haftet normalerweise nicht als Täter oder Mittäter

c) Unter bestimmten Umständen haftet der ISP als Anstifter. 129

d) Unter bestimmten Umstände haftet der ISP als Gehilfe 130

2. Die Situation, der ISP haftet als Störer

a) Unmittelbare Rechtsverletzung durch eine Drittperson ......... 132

b) Die Beurteilung des ISP als Störer.

c) Zumutbarkeit 133

d) Kenntnis. 133

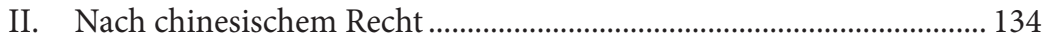

1. Der ISP hat prinzipiell keine allgemeine Prüfungspflicht ............... 134

2. Unter bestimmten Umstände haftet der ISP gesamtschuldnerisch mit seinen Nutzern.

a) Gesetzliche Grundlage ................................................................ 134

b) Der Grund der gesamtschuldnerischen Haftung des ISP ...... 135 
aa) Gesamtschuldnerische Haftung aufgrund

Gehilfenhaftung

bb) Gesamtschuldnerische Haftung aufgrund Mittäterhaftung.

cc) Gesamtschuldnerische Haftung wegen öffentlicher Politik

dd) Gesamtschuldnerische Haftung wegen Gefährdungshaftung

c) Die Auslegung der Kenntnisnahme i.S.v. $\$ 36$

Delikthaftungsgesetz

a) Die Sorgfaltspflicht des ISP als eine normale rationale Person für alle Informationen

bb) Spezielle Sorgfaltspflicht des ISP für die in besonderem Bereich der Webseite erscheinenden Informationen

cc) Die Qualifikation des ISP entscheidet über seine Kompetenz, Rechtsverletzungen zu entdecken.

dd) Die ISP in China haben wegen der Verwaltungsregelungen größere Möglichkeiten, um Rechtsverletzungen zu entdecken

ee) Die Größe der Sorgfaltspflicht des ISP kommt auch auf das dem Inhalt bezüglichen wirtschaftlichen Interesse an

ff) Volle Sorgfaltspflicht des ISP wegen ICP ähnlichem Verhalten ...

gg) Höhere Sorgfaltspflicht des ISP für die von dem gleichen Internetnutzer begangene wiederholte Rechtsverletzung...... 143

3. Unter bestimmten Umstände haftet der ISP als Gehilfe oder Anstifter.

3. Kapitel. Schutz des Rechtsverletzten 145

$\$ 11$ Maßnahmen zum Schutz des Rechtsverletzten gegen den rechtsverletzenden Internetnutzer

I Rechtsansprüche gegen den rechtsverletzenden Internetnutzer

1. Nach deutschem Recht 
a) Beseitigung- und Unterlassungsanspruch.................................. 147

b) Schmerzensgeldanspruch .......................................................... 147

c) Schadensersatzanspruch............................................................ 148

d) Antrag auf einstweilige Verfügung........................................... 148

2. Nach chinesischem Recht .............................................................. 149

a) Allgemeine Regelungen über die Ansprüche........................... 149

b) Schmerzensgeldanspruch .............................................................. 149

c) Antrag auf einstweilige Verfügung........................................... 150

II. Identifizierung des rechtsverletzenden Internetnutzers

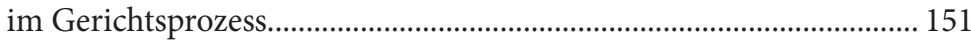

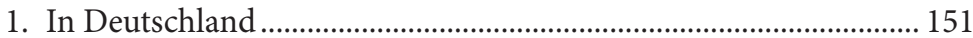

a) Für den Fall der Verletzung des Rechts am eigenen Bild .................................................................................. 151

aa) Identifizierung durch Anmeldedaten ......................................... 152

bb) Identifizierung durch IP-Adresse ................................................. 153

b) Für den Fall der Verletzung des Persönlichkeitsrechts

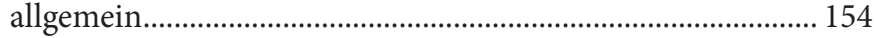

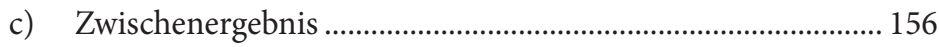

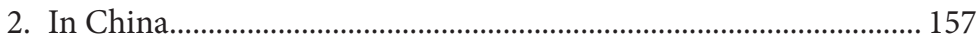

3. Ein kurzer Vergleich zwischen Deutschland und China ................. 158

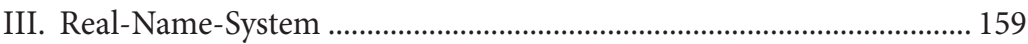

1. Real-Name-System in China .......................................................... 159

a) Die Entwicklung des Real-Name-System in China ................ 159

b) Die Verhältnismäßigkeit des Real-Name-System .................... 161

aa) Der legitime Zweck des Real-Name-System ............................ 161

bb) Die Geeignetheit des Real-Name-System................................... 161

cc) Die Erforderlichkeit des Real-Name-System .............................. 162

dd) Die Angemessenheit des Real-Name-System ............................. 163

(1) Bewertung über die Abschreckungsfunktion

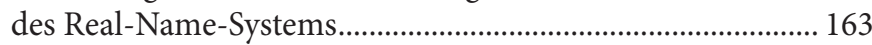

(2) Real-Name-System erhöht das Risiko vom Durchsickern der persönlichen Informationen ....................... 164

(3) Real-Name-System ermöglicht den Missbrauch der persönlichen Daten durch den Staat............................................ 165 
(4) Real-Name-System ist schädlich für die Erziehung des demokratischen Gedankens und die soziale Sicherheit ......... 166

(5) Das Vorbild von Südkorea ist als ein Fehler erwiesen............. 166

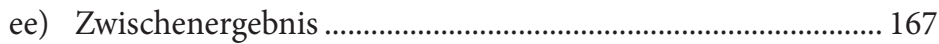

2. Real-Name-System in Deutschland.................................................. 167

a) Kein Grundrecht auf Anonymität ................................................ 167

b) Die Einschränkung der Anonymität im Internet...................... 168

3. Ein Vergleich zwischen Deutschland und China ............................ 170

$\$ 12$ Rechtsansprüche gegen den ISP als Störer ................................................. 171

I. Nach deutschem Recht...................................................................... 171

1. Die Reihenfolge der Ansprüche gegen den Internetnutzer als unmittelbarer Verletzer und den ISP als Störer........................... 171

2. Die Störerhaftung des ISP .................................................................... 172

a) Die Beseitigungs- und Unterlassungsansprüche fallen nicht unter die Haftungsprivilegierung

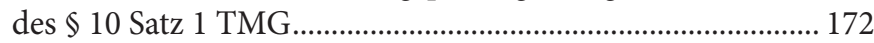

b) Beseitigungsanspruch ............................................................. 173

aa) Der Vorgang des Kommunikationsprozesses ............................. 174

bb) Die Kommentare über den Kommunikationsprozess ......................................................... 176

(1) Die Gefahr der extra entstehenden Pflichten und Kosten des ISP. 176

(2) Die Gefahr einer haftungsrechtlichen Zwickmühle

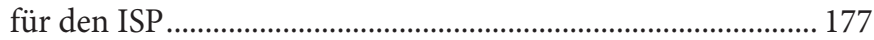

(3) Die Gefahr der Beschränkung der Meinungsfreiheit .............. 178

(4) Die Gefahr des ungenügenden Schutzes im Fall offensichtlicher Rechtsverletzung und der besonders schweren Persönlichkeitsverletzung.

(5) Der Kommunikationsprozess erfordert den Verzicht der Anonymität.................................................... 181

(6) Zusammenfassung über den Kommunikationsprozess .......... 181

c) Unterlassungsanspruch................................................................ 182

aa) Die Voraussetzungen eines Unterlassungsanspruchs gegen den ISP im Fall der Persönlichkeitsverletzung.............. 183 
(1) Wiederholungsgefahr und Erstbegehungsgefahr ..................... 183

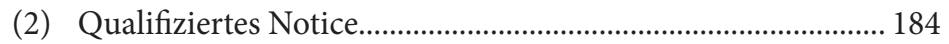

(3) Zumutbarkeit .......................................................................... 185

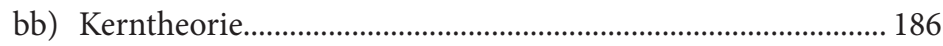

cc) Selbstaufspürung der Rechtsverletzung durch den Betroffenen .............................................................. 186

d) Schadensersatz- und Schmerzensgeldansprüche gegen den ISP wegen Pflichtverletzung ..................................... 187

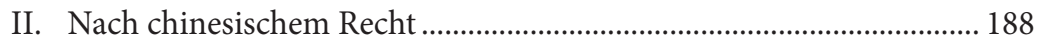

1. Die Reihenfolge der Ansprüche gegen den unmittelbaren rechtsverletzenden Internetnutzer und den ISP .............................. 188

2. Der Beseitigungsanspruch gegen den ISP ..................................... 190

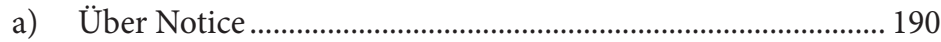

b) Über „Anti-Notice“.......................................................................... 191

c) Die Kommentare über das chinesische „Notice-and-Take-Down“-Verfahren ........................................... 193

aa) Die Gefahr einer haftungsrechtlichen Zwickmühle für den ISP

bb) Die Unfähigkeit des ISP bei der Beurteilung komplexer Persönlichkeitsrechtsverletzung ................................................. 194

cc) Die Möglichkeit, von der Quelle an die Rechtsverletzungen zu vermindern .......................................... 195

3. Der Unterlassungsanspruch gegen den ISP....................................... 196

4. Der Schadensersatzanspruch und der Schmerzensgeldanspruch gegen den ISP .......................................... 197

III. Eine Rechtsvergleichende Zusammenfassung ....................................... 198

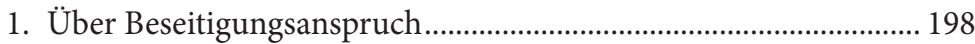
2. Über Schadensersatz- und Schmerzensgeldansprüche................... 198

3. Über Unterlassungsanspruch ............................................................... 199

$\$ 13$ Die anderen Maßnahmen zum Schutz des Rechtsverletzten während der Menschenfleischsuche .............................. 201

I. Ansprüche gegen den ICP und ISP als Täter oder Mittäter ................. 201

II. Selbstregulierung durch Internetnutzer............................................. 201 


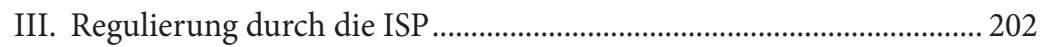

IV. Einleitung der Menschenfleischsuche von Journalisten oder zuständigen Behörden........................................................................ 204

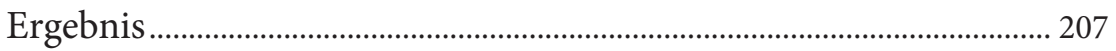

$\$ 14$ Zusammenfassung und Schlussfolgerungen............................................... 209

I. Zusammenfassung ................................................................................... 209

II. Die Zukunft der Menschenfleischsuche............................................. 213

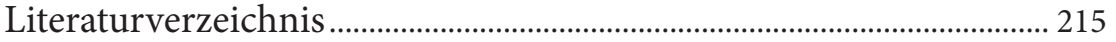





\section{Abkürzungsverzeichnis}

a.F.

Abs.

AfP

AGZR

Anm.

Art.

Aufl.

$\mathrm{BB}$

BBS

BDSG

bearb.

Beschl. v.

BfDI

BGB

$\mathrm{BGH}$

BGHSt

BGHZ

BVerfG

BVerfGE

bzw.

CNNIC

CR

CSDN

DMCA

DuD alte Fassung

Absatz

Archiv für Presserecht - Zeitschrift für Medien- und Kommunikationsrecht

Allgemeine Grundsätze des Zivilrechts

Anmerkung

Artikel

Auflage

Betriebsberater

Bulletin Board System

Bundesdatenschutzgesetz

bearbeitet

Beschluss von

Bundesbeauftragte für den Datenschutz und die Informationsfreiheit

Bürgerliches Gesetzbuch

Bundesgerichtshof

Entscheidungen des Bundesgerichtshofs in Strafsachen

Entscheidungen des Bundesgerichtshofs in Zivilsachen

Bundesverfassungsgericht

Entscheidungen des Bundesverfassungsgerichts

beziehungsweise

China Internet Network Information Center

Computer und Recht

Chinese Software Developer Network

Digital Millenium Copyright Act

Datenschutz und Datensicherheit 
EG

EGMR Europäischer Gerichtshof für Menschenrechte

EU

$\mathrm{EuGH}$

GG

ggf.

GRUR

GRURInt

GRUR-RR

i.S.v.

i.V.m.

ICP

ID

IP

ISP

jurisPR-ITR

$\mathrm{JuS}$

$\mathrm{K} \& \mathrm{R}$

KG

KunstUrhG

LAN

LMK

MMR

n.F.

NJOZ

Europäische Union

Europäischer Gerichtshof

Grundgesetz

gegebenenfalls

Gewerblicher Rechtsschutz und Urheberrecht

Gewerblicher Rechtsschutz und Urheberrecht, Internationaler Teil

Gewerblicher Rechtsschutz und Urheberrecht, Rechtsprechungs-Report

im Sinne von

in Verbindung mit

Internet Content Provider

Identification

Internet Protocol

Internet Service Provider

Juris PraxisReport IT-Recht

Juristische Schulung

Kommunikation \& Recht

Kammergericht

(Gesetz betreffend das Urheberrecht an Werken

der bildenden Künste und der Photographie)

Kunsturheberrechtsgesetz

Local Area Network

Lindenmaier-Möhring - Kommentierte

BGH-Rechtsprechung

Multimedia und Recht

neue Fassung

Neue Juristische Online Zeitschrift 
NJW

NJW-Beil.

NJW-RR

No.

$\mathrm{Nr}$.

NStZ

NVwZ

NZV

OLG

OVG

PRC

RL

Rn.

RStV

S.

StGB

TDG

TKG

TMG

UrhG

Urt. v.

USA

VeRI

vgl.

vol.

VuR

WRP

z.B.

ZD
Neue juristische Wochenschrift

Neue Juristische Wochenschrift-Beilage

NJW-Rechtsprechungs-Report

Number

Nummer

Neue Zeitschrift für Strafrecht

Neue Zeitschrift für Verwaltungsrecht

Neue Zeitschrift für Verkehrsrecht

Oberlandesgericht

Oberstes Volksgericht (der V. R. China)

People‘s Republic of China

Richtlinie

Randnummer

Staatsvertrag für Rundfunk und Telemedien

Seite

Strafgesetzbuch

Teledienstegesetz

Telekommunikationsgesetz

Telemediengesetz

Urheberrechtsgesetz

Urteil von

United States of America

Verifizierte Rechteinhaber

vergleiche

volume

Verbraucher und Recht

Wettbewerb in Recht und Praxis

zum Beispiel

Zeitschrift für Datenschutz 
$\mathrm{ZPO}$

ZUM

ZUM-RD
Zivilprozessordnung

Zeitschrift für Urheber-und Medienrecht

Zeitschrift für Urheber-und

Medienrecht-Rechtsprechungsdienst 


\section{Einleitung}





\section{$\$ 1$ Menschenfleischsuche als ein soziales Phänomen}

\section{Die originale Bedeutung der Menschenfleischsuche in China}

Google und Bing sind die üblichen Suchmaschinen im Internet. Die Suche durch solche Suchmaschine erfolgt durch Eingabe von Schlagwörtern. Hinter solcher Suche stehen komplizierte Algorithmen, Computersprachen und andere ITMethoden. Die Suchergebnisse sind ein Ergebnis der Dialoge zwischen Personen und Computer.

Menschenfleischsuche wird durch „Menschenfleischsuchmaschine“ verwirklicht. Der Begriff von Menschenfleischsuchmaschine hebt erst die Wörter "Menschen“ und „Suchmaschine“ hervor. Eine Menschenfleischsuchmaschine funktioniert prinzipiell wie die oben genannten Suchmaschinen im Internet. Aber diese Suche wird nicht durch Algorithmen oder IT-Methoden sondern durch Menschen verwirklicht. Wenn ein Internetnutzer ein Problem hat, stellt er zur Lösung dieses Problems eine Frage ins Internet. Diese Frage wird von anderen Internetnutzern beantwortet. Das bedeutet, dass die anderen Internetnutzer die Rolle der Suchmaschine spielen. Die gefundenen Suchergebnisse sind dann ein Ergebnis der Dialoge zwischen einzelnen Personen und mehreren Personen. Das Internet selbst stellt nur die Plattform für diese Dialoge dar. Der Suchprozess durch Menschenfleischsuchmaschinen wird als Menschenfleischsuche bezeichnet.

Menschenfleischsuchmaschine bedeutet tatsächlich „Menschen als Suchmaschine“. Das Wort „Menschenfleisch“ zeigt den mit Fleisch und Blut gebauten Körper eines Menschen. Das Wort benutzt man, um mit der Suche durch aus Metall gebaute Maschine zu unterscheiden. Übrigens zeigt das Wort „Menschenfleisch“ auch die blutige Seite der Menschenfleischsuche. Dies wird nachher erklärt.

Man kann sagen, dass Menschenfleischsuche nicht anders als ein FragenAntworten-Dialog im Alltagsleben ist. Der Unterschied ist nur, dass dieser Dialog im Internet durchgeführt wird und unzählig viele Personen daran teilnehmen können.

Die Menschenfleischsuche in China stammt aus einem Internetforum mit dem Namen „Mop“. Die zurzeit bekanntesten Menschenfleischsuche-Webseiten sind „Baidu Knows“, „iask von Sina“, „Tianya Answers“ usw. Um die Internetnutzer an der Teilnahme der Menschenfleischsuche zu motivieren, benutzen die oben genannten Webseiten häufig ein virtuelles Belohnungssystem. Im Mop z.B. trägt der Fragesteller eine Menge von „virtuellem Geld“ als Belohnung für 
die beste Antwort bei. Mit diesem virtuellen Geld können die Internetnutzer im Mop einige Privilegien genießen. Im Fall der hinreichenden Teilnahme der anderen Internetnutzer werden die genauen, komplexen Informationen als Antwort dem Fragesteller eingerichtet. ${ }^{1}$

Der Schwerpunkt der Menschenfleischsuche ist es, alle Kräfte der Internetnutzer zu bündeln, um ein Problem zu lösen. ${ }^{2}$ Wegen der Interaktion der Internetnutzer und der multiplen Quellen der gesammelten Informationen wird durch Menschenfleischsuche schneller und genauer ein Suchergebnis erzielt. Die Suchfähigkeit ist besser als jeder anderen von Technik unterstützten Suchmaschinen. ${ }^{3}$

Übrigens braucht es für die Fragestellung keine Schlagwörter wie für den Fall der Suche durch Suchmaschine. Die Fragen können durch Sätze und Texte gestellt werden. Im Vergleich mit der Suche durch Suchmaschinen werden die Fragen besser verstanden.

\section{Die geänderte Bedeutung der Menschenfleischsuche in China}

Seit dem Fall von „Chen Ziyao aus Microsoft" ist Menschenfleischsuche von Kenntnissuche zu Personensuche geändert. Im Jahr 2001 hat ein Internetnutzer im Mop ein Bild von einer bildhübschen Frau hochgeladen und gesagt, dass die Frau seine Freundin ist. In kurzer Zeit nach der Veröffentlichung dieses Bildes haben einige andere Internetnutzer die Frau erkannt und gesagt, dass die Frau tatsächlich die Markenbotschafterin vom Unternehmen Microsoft ist, die Chen Ziyao heißt und auf keinen Fall seine Freundin sei. Um ihre Behauptung zu beweisen, haben die anderen Internetnutzer nach und nach die persönlichen Informationen und andere Bilder von Chen Ziyao im Mop offengelegt.

Das Ereignis, das die Entwicklungsrichtung der Menschenfleischsuche völlig geändert hat, ist die Menschenfleischsuche nach einer Frau, die eine Katze misshandelt und umgebracht hat. Das Ereignis lief wie Folgt:

Internetnutzer A hat am 28.02.2006 eine Bilderserie, die aus einem Video geschnitten wurde, im Internet veröffentlicht. Die Bilder zeigen eine gut gekleidete Frau, die eine Katze gewaltsam misshandelt und zum Schluss brutal getötet hat. Diese Bilderserie hat sofort den Zorn der anderen Internetnutzer entzündet. In kurzer Zeit hat Internetnutzer B diese Bilder an Mop weitergeleitet. Ein anderer

1 Wang Chengwei, Journal of Public Management 2011, No. 4, 21, 23.

2 Zhang Bing, Journal of Heilongjiang Administrative Cadre Institute of Politics And Law 2010, No. 1, 51.

3 Su Zhe, Hebei Law Science 2012, No. 2, 26, 29. 
Internutzer $\mathrm{C}$ hat die Bilder weiterhin auf einer der bekanntesten Blogwebseite in China "Tianya" veröffentlicht, und dazu noch einen "Steckbrief“ ausgelöst, um die tatsächliche Identität der Frau herauszufinden. Danach haben viele Internetnutzer freiwillig Kopfgeld beigetragen, um die Internetnutzer zu belohnen, die wertvolle Informationen über die Frau anbieten konnten. Am 02.03.2006 hat Internetnutzer D festgestellt, dass die Misshandlung der Katze in der kleinen Stadt X stattgefunden hat. Diese Information war der Wendepunkt der Suche. Zwei Tage später waren als Täter der Misshandlung die Frau und zwei andere Mittäter identifiziert. Alle ihrer persönlichen Informationen einschließlich Namen, Adresse, Telefonnummer, Arbeitgeber usw. waren im Internet veröffentlicht. Die Suche hat insgesamt 6 Tage gedauert, und war offensichtlich schneller als die Suche durch die Polizei. Nachdem die drei Personen identifiziert waren, bekamen sie ständig Anrufe von fremden Leuten, die sie aufgrund ihres Verhaltens beschimpften. Zum Schluss war auch die Polizei mit dem Fall beschäftigt. Die Betroffenen mussten nach Verlangen der Polizei einen Entschuldigungsbrief schreiben und veröffentlichen, um sich zu entschuldigen. Wegen dieses Ereignisses sind diese drei Personen von ihren Arbeitgebern entlassen worden.

Seit diesem Ereignis hat sich das Modell der Menschenfleischsuche geändert. Die Suche zielt nicht mehr darauf, normale Kenntnisse zu erlangen, sondern viel häufiger um eine Person zu identifizieren. In dieser Arbeit wird Menschenfleischsuche eher im Sinne von Personensuche diskutiert.

\section{Die in dieser Arbeit zu diskutierende Menschenfleischsuche}

\section{Die Motivationen der Menschenfleischsuche und die Beispiele in China}

Für die Regulierung der Menschenfleischsuche spielt die Motivation eine entscheidende Rolle. Zum Beispiel soll eine Suche um Korruption zu entdecken völlig anders als eine Suche um den Ex-Freund zu rächen behandelt werden. Deswegen ist es nötig, die Menschenfleischsuche nach Motivationen zu sortieren.

\section{a) Um Korruption oder anderes rechtswidriges Verhalten von Beamten oder anderen Prominenten aufzudecken}

Solche Menschenfleischsuche zielt darauf, das Verhalten der Beamten oder der Behörden zu überwachen. ${ }^{4}$ Durch diese Suche wird häufig Korruption ent-

4 Zheng Gencheng, Morality and Civilization 2010, No. 5, $121 \mathrm{ff}$. 
deckt. Die Motivation gilt auch für die Überwachung der Prominenten, um zu kontrollieren, ob sie unter Verwendung ihrer besonderen sozialen Stellungen rechtswidriges wirtschaftliches Interesse erwerben. ${ }^{5}$ Ein typisches Beispiel für solche Suche ist der Fall von "Jiu-wu-zhi-zun“ in Nanjing. ${ }^{6}$

"Jiu-wu-zhi-zun“ ist die teuerste Luxus-Zigaretten-Marke in Nanjing, die sich die normalen Bürger nicht leisten können. Ende 2008 hat Zhou Jiugeng, der Direktor der Immobilienbehörde von einem Bezirk in Nanjing, ungeeignete amtliche Entscheidung bezüglich des Immobilienmarktes getroffen, der wegen der hohen Preise der Immobilien seit langem im Fokus der Bürger steht. Wegen dieser ungeeigneten Entscheidung war Zhou Jiugeng die Zielperson der Menschenfleischsuche geworden. Ein Bild von ihm bei einer Tagung war im Internet veröffentlicht. Auf dieser Bild lag eine Packung „Jiu-wu-zhi-zun“ auf dem Tisch, während er eine dicke Armbanduhr von „Vacheron Constantin“ trägt, die mehr als 12,000 Euro kostet. Es wurde auch von dem Internetnutzer ausgerechnet, ob er sich mit seinem normalen Entgelt dieses Luxusleben leisten kann. Das Ergebnis ließ Korruption vermutet. Wegen der Menschenfleischsuche wurde die Polizei eingesetzt. Zhou Jiugeng wurde wegen Korruption verhaftet, und für 11 Jahre Freiheitsstrafe verurteilt. $^{7}$

\section{b) Um rechtswidriges Verhalten normaler Bürger aufzudecken}

Solche Menschenfleischsuche hat zum Ziel, die Person, die rechtswidriges Verhalten begangen hat, zu identifizieren, und möglicherweise die zuständige Behörde darüber zu informieren, um die Person zu bestrafen. ${ }^{8}$ Dazu gehört das Beispiel der Misshandlung der Katze, das oben als Wendepunkt der Menschenfleischsuche genannt wurde.

\section{c) Um gegen unmoralisches Verhalten zu kämpfen}

Solche Menschenfleischsuche zielt auf den Schutz der traditionellen chinesischen moralischen Gerechtigkeit. Es geht besonders um die Angelegenheiten, die vom Gesetz nicht erfasst werden, die aber der chinesischen traditionellen Moral nicht genügen können.

5 Su Zhe, Hebei Law Science 2012, No. 2, 26, 28.

6 Liu Han, Peking University Law Journal 2011, 870, 871.

7 Wikipedia, http://zh.wikipedia.org/wiki/\%E5\%91\%A8\%E4\%B9\%85\%E8\%80\%95 (besucht am 04.04.2015)

8 Zheng Gencheng, Morality and Civilization 2010, No. 5, 121ff.; Liu Han, Peking University Law Journal 2011, 870, 871. 
Ein Beispiel ist aus dem August 2012 wegen Übergabe eines Sitzplatzes im Bus für eine Frau mit einem kleinen Kind im Arm. Die Internetnutzerin „ljktdd“ hat im Bus gesehen, dass ein im Bus sitzendes 20-jähriges Mädchen die direkt neben ihr stehende Frau, die ein Kind im Arm hat, völlig vernachlässigte, obwohl die Ticketverkäuferin mehrmals die Fahrgäste gebeten hat, für die Frau einen Sitzplatz freizumachen. Das Verhalten des Mädchens hat „ljktdd“ geärgert. Sie hat dann mit der Kamera ihres Mobiltelefons Lichtbilder von dem Mädchen mit direkter Sicht auf ihr Gesicht aufgenommen und sofort im Internet offengelegt, um eine Menschenfleischsuche gegen das Mädchen auszulösen. Diese Veröffentlichung wurde innerhalb eines Tages $33000 \mathrm{Mal}$ gelesen und weitergeleitet. Das Mädchen wurde von zahlreichen Internetnutzern beschimpft. ${ }^{9}$

Häufiger passiert die Menschenfleischsuche wegen sexueller oder familienbezüglicher Moral, wie z. B. Untreue zwischen Partnern, Misshandlung gegen ältere Familienmitglieder. Als Beispiel hierfür ist der Fall von „Wang Fei und Jiang Yan“, der zu der sogenannten ersten Entscheidung über Menschenfleischsuche geführt hat. ${ }^{10}$

Frau Jiang Yan ist die Ehefrau von Herrn Wang Fei. Seitdem sie wusste, dass er eine Mätresse hat, und seine Familie die Mätresse sogar akzeptiert hat, hat sie in ihrem MSN-Blog in der Form eines Tagebuches ihren Gedanken über die erfolgloses Ehe bis zu dem letzten Tag ihres Lebens erzählt, an dem sie Selbstmord begangen hat. In ihrem Blog hat sie den realen Name und die Adresse des Arbeitsgebers von Wang Fei erwähnt und auch ein Bild von Wang Fei und seiner Mätresse hochgeladen. Ihr Blog war jedoch mit Passwort gesichert. Erst kurz vor ihrem Selbstmord wurde das Passwort an einen Internetnutzer weitergegeben, der es dann der Schwester von Jiang Yan weiter gegeben hat.

Ein alter Freund von Jiang Yan, der Zhang Leyi heißt, hat nach dem Selbstmord von Jiang Yan eine Webseite registriert. Darauf haben er und die Schwester von Jiang Yan Texte zum Andenken an Jiang Yan geschrieben und die Inhalte des Tagesbuches von Jiang Yan veröffentlicht. Dazu gehören die persönlichen Informationen von Wang Fei einschließlich seines Namens und der Adresse seines Arbeitsgebers und das Bild von Wang Fei und seiner Mätresse. Zhang Leyi hat übrigens seine Webseite auch mit anderen bekannten Blogwebseite wie z.B. „Tianya“, „Sina“ verlinkt.

9 Wang Wei/Wang Luyao, Chongqing shang bao, 17.08.2012, http://news.sina.com. $\mathrm{cn} / \mathrm{s} / 2012-08-17 / 152924987638 . s h t m l$ (besucht am 04.04.2015).

10 Liu Han, Peking University Law Journal 2011, 870, 871. 
Die Geschichte ist im Internet sofort bekannt geworden. Das untreue Verhalten von Wang Fei und das schlechte Verhalten seiner Familien gegen Jiang Yan haben den Zorn der Online-Community zugezogen. Auf „Tianya“ war eine Menschenfleischsuche gegen Wang Fei gestartet worden. Durch die Suche wurden noch mehr persönliche Informationen von Wang Fei veröffentlicht. Dazu gehört seine Familienadresse. Die aufgeregten Internetnutzer waren zu seiner Familien gegangen, haben Plakate mit Schimpfwörtern auf die Tür und auf die Wände geklebt. Der Arbeitgeber von Wang Fei musste seine Arbeit auch vorläufig einstellen.

Wang Fei hat wegen Ehrverletzung ${ }^{11}$ gegen Zhang Leyi bzw. seine Webseite, den ISP von „Tianya“ und den ISP von „Daqi“, der für diese Geschichte eine Sonderthema-Webseite eingerichtet hat und Kommentare offen zusammengestellt hat, vor Gericht geklagt. Die beide Instanzen haben entschieden, dass die Ehrverletzung bzw. die Verletzung der Privatsphäre von Zhang Leyi und „Daqi“ begründet sind, während die Rechtsverletzung von „Tianya“ nicht begründet ist, weil er das Notice-Take-Down-Verfahren verfolgt hat. ${ }^{12}$

\section{d) Um die Person in schwieriger Situation zu helfen}

Solche Menschenfleischsuche ist meistens von einem warmherzigen Internetnutzer initiiert, um Leute mit schwierigem Leben oder in schwieriger Situation zu helfen oder zu unterstützen. ${ }^{13}$

Ein Beispiel wäre die Menschenfleischsuche nach dem Erdbeben in Sichuan von 2008. Wegen des Erdbebens haben viele Personen den Kontakt mit ihren Familien verloren. Die Menschenfleischsuche hat damals dazu geführt, vermisste Personen wieder zu finden. ${ }^{14}$

\section{e) Um Rache auszü̈ben}

Eine andere Menschenfleischsuche kann erfolgen, um eine Person zu rächen, die mit dem Veranlasser der Menschenfleischsuche persönlich in Streit oder in

11 Damals wird der Schutz der Privatsphäre noch vom Ehrenschutz einbezogen.

12 Chaoyang Unteres Volksgericht, Urt. v. 16.12.2008 - (2008) chao min chu zi di 10930 hao; Peking Zweites Mitteleres Volksgericht, Urt. v. 23.12.2009 - (2009) er zhong min zhong zi di 5603 hao.

13 Zheng Gencheng, Morality and Civilization 2010, No. 5, 121ff.; Su Zhe, Hebei Law Science 2012, No. 2, 26, 28.

14 Li Fang, Shi chang bao, 17.06.2008, http://news.xinhuanet.com/focus/2008-06/17/ content_8384135.htm (besucht am 04.04.2015). 
Kollision geraten ist, weil durch die Veröffentlichung der persönlichen Daten der Zielperson ihr Leben gestört wird. ${ }^{15}$

Im August 2007 hat ein Manager im Name der Firma eine Menschenfleischsuche nach ihrem ehemaligen Arbeitnehmer veranstaltet. Es wurde bei der Motivation der Suche gesagt, dass der ehemalige Arbeitnehmer Vermögen von der Firma entwendet haben sollte. Diese Behauptung war von diesem Manager wider besseren Wissens erhoben worden, der mit dem Arbeitnehmer in Streit geraten war. Der Manager wurde von dem Gericht wegen Ehrverletzung gegen den ehrmaligen Arbeitnehmer verurteilt.

\section{f) Einfach aus Neugier oder zum puren Spaß}

Eine weitere Variante der Menschenfleischsuche ist möglich, um die Neugier des Veranlassers auf die Zielperson zu befriedigen ${ }^{16}$, oder auch nur um Spaß zu haben oder andere Internetnutzer zu amüsieren. ${ }^{17}$

Am 16.07.2009 hat eine Nachricht in einem Forum über das Online-Spiel „World of Warcraft" die Aufmerksamkeit der Internetnutzer gewonnen. Die Nachricht lautet „Jia Junpeng, deine Mutter ruft dich, zum Essen nach Hause zu gehen“. In 6 Stunden wurde diese Nachrichte 390,617 Mal gelesen und 17,000 Mal darauf geantwortet. In den kommenden Tagen bekam diese Nachrichten zusammen 7,818,740 Leser und 302,259 Antworten. Das Ereignis wurde als „Internet-Wunder“ bezeichnet. Zur Neugier wurde Jia Junpeng durch Menschenfleischsuche von den Internetnutzern gesucht. Die Suche hatte multiple Ergebnisse gehabt.

Aufgrund des durch das Ereignis entstehenden potenzialen wirtschaftlichen Wertes haben sich Unternehmen nach und nach eingesetzt, zu behaupten, dass das Ereignis eine geplante Werbung war. Die Wahrheit kann man leider nicht feststellen. Jedoch war der Ausdruck „Zum Essen nach Hause gehen“ einer der bekanntesten Internetausdrücke geworden. ${ }^{18}$

\section{g) Zum Zweck der Werbung}

Solche Menschenfleischsuche ist tatsächlich versteckte Werbung in Form von Menschenfleischsuche. Die ganze Suche wurde von kommerziellen Instituten

15 Zheng Gencheng, Morality and Civilization 2010, No. 5, $121 \mathrm{ff}$.

16 Tian Feilong, Internet Law Review 2009, 80, 83.

$17 \mathrm{Su}$ Zhe, Hebei Law Science 2012, No. 2, 26, 28; Zheng Gencheng, Morality and Civilization 2010, No. 5, $121 \mathrm{ff}$.

18 Wikipedia, http://en.wikipedia.org/wiki/Jia_Junpeng (besucht am 04.04.2015). 
geplant. Der Ablauf der Suche wurde auch von den Instituten kontrolliert, damit ein gezieltes Ergebnis gewonnen werden könnte. Es ist eine Strategie des Marketings. ${ }^{19}$

Ein typisches Beispiel wäre der Fall von „Lan Dong jie jie“. ${ }^{20}$ Seit April 2008 erscheint im Internet eine Frau Lan Dong, die gerne die Begrenzung der Toleranz der Internetnutzer herausfordert. Sie protzte mit ihrem Reichtum, teures Auto, Top-Marken-Tasche, Villa usw. Gleichzeitig erniedrigt sie als eine in den 70er Jahren geborene die in 80er und 90er Jahren geborene Generation. Ihre übertreibende Gestik und Mimik in den von sich hochgeladene Bildern haben sie sehr leicht zur Zielperson der Menschenfleischsuche gemacht. Aber nach der Suche haben die Internetnutzer festgestellt, dass sie nur eine erfundene Figur von der Firma WOYO ist. Die Informationen über Lan Dong kommen alle original von der Webseite von WOYO. Die Suche war eine geplante Werbung für die Webseite. ${ }^{21}$

Ähnliches gibt es auch bei Personen, die sich durch Menschenfleischsuche bekannt machen wollen. Solche Personen sind meistens aus der Vergnügungsbranche oder wollen in der Zukunft in der Vergnügungsbranche arbeiten. Häufig wird eine Firma beauftragt diese Suche vorzubereiten und durchzuführen.

Ein Beispiel wäre der Fall von „der hübschesten Putzfrau“, die tatsächlich eine Musikstudentin ist, die schon mal eine unbedeutende Rolle im Film gespielt hatte. Sie hat als Putzfrau in einer Automobilausstellung gearbeitet und wurde von einem Kameramann während der Arbeit „heimlich“ fotografiert, weil sie als Putzfrau sogar hübscher als die Models war. Durch die Menschenfleischsuche wurde sie schnell bekannt. Mit der Vertiefung der Suche ist es deutlich geworden, dass der Kameramann tatsächlich ein bekannter „Internet-Star-Maker“ war und die Menschenfleischsuche eine geplante Werbung war. ${ }^{22}$

In dieser Arbeit wird nur die Menschenfleischsuche, die ohne Einwilligung der Zielperson angeschaltet ist, diskutiert. Die Menschenfleischsuche zum Ziel der Werbung gehört nicht dazu.

19 Zheng Gencheng, Morality and Civilization 2010, No. 5, 121ff.; Su Zhe, Hebei Law Science 2012, No. 2, 26, 28.

20 Zheng Gencheng, Morality and Civilization 2010, No. 5, $121 \mathrm{ff}$.

21 Baike,http://www.baike.com/wiki/\%E5\%85\%B0\%E8\%91\%A3\%E5\%A7\%90\%E5 $\%$ A7\%90 (besucht am 04.04.2015).

22 Sou hu she qu bian ji bu, http://hot.club.sohu.com/s2008/chezhanzuimeiqingjiegong/ (besucht am 04.04.2015). 


\section{Der Vorgang der in dieser Arbeit zu diskutierende Menschenfleischsuche}

Aufgrund der oben genannten Beispiele wird im Folgenden versucht, den Vorgang einer Menschenfleischsuche im Sinne dieser Arbeit zu generalisieren.

Für eine Menschenfleischsuche ist die Motivation der erste Schritt. Wenn eine der oben genannten Motivationen besteht, würde einer oder mehrerer der Motivierten herausspringen, um eine Suche gegen die dem Ereignis bezogene Person einzuleiten. Der Motivierte wird im Folgenden als Veranlasser der Menschenfleischsuche oder „Veranlasser“ genannt. Die gesuchte Person wird Zielperson der Menschenfleischsuche oder „Zielperson“ genannt.

Der Veranlasser würde normalerweise eine Geschichte über die Zielperson und ihres Verhalten erzählen, um die Notwendigkeit der Menschenfleischsuche gegen die Zielperson zu begründen, bzw. den anderen Internetnutzer zur Teilnahme der Suche zu motivieren. Dazu würde der Veranlasser alle Informationen, die er über die Zielperson weiß, veröffentlichen, um den anderen Internetnutzern schon einige Hinweise für die weitere Suche zu geben. Die Hinweise sind meistens in Form von Bildnis oder Video der Zielperson. Ausnahmsweise können die Hinweise auch ein Name oder eine Beschreibung über die Zielperson sein. Es wird von dem Veranlasser vorgeschlagen, die Informationen über die Zielperson so viel wie möglich im Internet einzutragen, um die Zielperson im realen Leben zu identifizieren.

Für die Menschenfleischsuche wird eine Internetplattform benötigt. Die größten bzw. bekannten Internetportale, Blogs, Internetforen, oder sozialen Netzwerke sind wegen der hohen Zugriffshäufigkeit von dem Veranlasser besonders beliebt. Der Betreiber solcher Internetplattform sind „Internet Service Provider" (Internetdienstanbieter). Im Folgenden werden sie als ISP genannt. Der ISP bietet nur Dienst im technischen Sinne. Die auf seiner Webseite stehenden Inhalte sind von den Internetnutzern eingetragen.

Für den Fall, dass die Webseite die Inhalte selbst einträgt, wird der Dienstanbieter als „Internet Content Provider“ (ICP) genannt. Die von Zhang Leyi be-

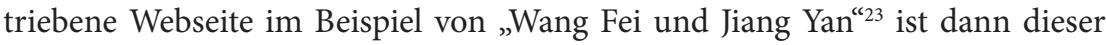
Fall. Der genaue Unterschied zwischen ICP und ISP im Sinne dieser Arbeit wird unten im $\$ 2$, I, 5 erklärt.

Nachdem der Veranlasser die Suche eingeleitet hat, erwartet er viele Eintragungen von anderen Internetnutzern über die Zielperson. Die Internetnutzer, die die Informationen über die Zielperson eingetragen haben, werden Informationseintragende genannt.

23 Siehe oben unter $\$ 1$ III $1 \mathrm{c})$. 
Das Erlangen der Informationen über die Zielperson ist durch zwei Wege ermöglicht. Der erste Weg ist mit Benutzung der normalen Suchmaschine (wie z.B. Google) und anderer Internetwebseite (wie z.B. soziales Netzwerk wie Facebook) die im Internet schon veröffentlichten Informationen zu sammeln, zu sortieren, zu analysieren, zusammenzufassen und die zusammengefassten Ergebnis in dem Forum einzutragen, wo die Menschenfleischsuche stattfindet. Solcher Informationseintragender wird "Informationssammler" genannt.

Der zweite Weg zur Erlangung der Informationen über die Zielperson erfolgt durch die Internetnutzer, die mit der Zielperson bekannt, befreundet oder sogar verwandt sind. Die von ihnen berichteten bzw. eingetragenen Informationen der Zielperson waren noch nie im Internet veröffentlicht. Solcher Informationseintragender wird "Informationsberichter" genannt.

Außer den Informationseintragenden gibt es auch eine Menge von Internetnutzer, die nur die Zielperson und ihr Verhalten kommentieren. Solcher Internetnutzer wird „Kommentator" genannt.

Die personenbezogenen Informationen der Zielperson werden nach und nach veröffentlicht und zusammengefasst, bis zum Schluss - bis seine Identität im normalen Leben bekannt ist. Das ist das Ende der "Suchphase“ der Menschenfleischsuche. Durch diese Phase wird das offline Leben der Zielperson online offengelegt.

Danach kommt die zweite Phase der Menschenfleischsuche, nämlich „die Phase nach der Suche“. ${ }^{24}$ In dieser Phase wird die Zielperson mit Namensnennung Person spezifisch kommentiert. Wegen der Bekanntmachung der Telefonnummern und der Anschriften ist es in dieser Phase möglich, dass die Internetnutzer die Zielperson persönlich kontaktieren. Das Kontaktieren erscheint jedoch meistens als Stören des normalen Lebens der Zielperson. ${ }^{25}$

\section{Der Unterschied zwischen Menschenfleischsuche und anderen ähnlichen Phänomen}

\section{Der Unterschied mit Suchmeldung}

Menschenfleischsuche und die Suchmeldung im traditionellen Sinne haben einige Gemeinsamkeiten aber hauptsächlich Unterschiede. Die Unterschiede

24 Yu Xiaohai, Journal of Dezhou University 2009, No. 3, 90, 92.

25 Vgl. Yu Xiaohai, Journal of Dezhou University 2009, No. 3, 90, 92; Deng Xiaoxia/Wang Shuhuai, Renmin Ribao 10.08.2007; und Wu Bo, S. 14. 
ergeben sich aus der Motivation und dem Ziel der Suchmeldung. Der Veranlasser der Suchmeldung ist meistens Familienmitglied oder Verwandter der zu suchenden Person. Die zu suchende Person zu finden ist das einzige Ziel der Suche. Und das Ziel ist für die Zielperson meistens positiv. Für die Menschenfleischsuche gibt es, wie oben aufgelistet wurde, unterschiedliche Motivationen. Nicht alle dieser Motivationen sind zugunsten der Zielperson. Im Gegensatz zur Suchmeldung führen die meisten Menschenfleischsuchen zur Rechtsverletzung der Zielperson.

Ein essentieller Unterschied zwischen Menschenfleischsuche und Suchmeldung ist es jedoch, dass der Veranlasser der Suchmeldung die Zielperson kennt, und die hauptsächlichen persönlichen Informationen der Zielperson hat. Im Gegensatz kennt der Veranlasser der Menschenfleischsuche die Zielperson normalerweise nicht. Die Zielperson der Menschenfleischsuche soll identifiziert werden. Deswegen ist auch die im Internet gestellte Suchmeldung nicht die Menschenfleischsuche im Sinne dieser Arbeit.

\section{Der Unterschied mit der Öffentlichkeitsfahndung im Internet}

Menschenfleischsuche ist auch anders als die Öffentlichkeitsfahndung mit Belohnung im Internet. Die Öffentlichkeitsfahndung wird von der zuständigen Behörde der öffentlichen Sicherheit oder Staatsanwaltschaft zugestimmt und durchgeführt. Obwohl der Verdächtigte manchmal auch die Zielperson der Menschenfleischsuche ist, ist die Feststellung der Verdächtigten für online Öffentlichkeitsfahndung viel strenger, weil sie im weitesten Sinne zum Strafrechtsprozess gehört. Das prozessbezügliche Recht der Verdächtigten muss bei der online Öffentlichkeitsfahndung berücksichtigt werden. Im Gegensatz dazu wird Menschenfleischsuche von normalen Bürgern veranstaltet. Das Recht der Zielperson wird häufig vernachlässigt.

Übrigens werden die Informationen über den Verdächtigten bei der Öffentlichkeitsfahndung direkt der Behörde der öffentlichen Sicherheit mitgeteilt, während die Informationen über die Zielperson bei der Menschenfleischsuche im Internet offengelegt werden.

\section{Der Unterschied mit dem Klatsch im Alltagsleben}

Menschenfleischsuche ist auch nicht völlig identisch mit dem Klatsch im offline Leben. Klatsch ist regional und zeitlich begrenzt. Erstens erfolgt die Verbreitung der Informationen mittels Klatsch durch die Erzählung von Mund zu Mund. Dies hat zur Folge, dass sich der Verbreitungsbereich häufig auf kleine 
Gruppen und Bekannte beschränkt. ${ }^{26}$ Zweitens kommt es bei der Verbreitung der Informationen durch Klatsch auf die Erinnerung an. Deswegen wird der Inhalt des Klatsches nach einiger Zeit unvermeidlich von den Menschen vergessen.

Im Fall von Menschenfleischsuche können die Interessierten die Informationen der Zielperson, die im Internet veröffentlicht sind, weltweit jeder Zeit unbegrenztes Mal abrufen, kopieren und verbreiten. Die Informationen werden nie wieder wirklich permanent vom Internet gelöscht. Im Zeitalter des Internet ist die Situation des menschlichen Gedächtnisses geändert. Vergessen wird zur Ausnahme und permanentes Erinnern wird dagegen normal. ${ }^{27}$

\section{Der Unterschied mit der Personensuchmaschine}

Die Menschenfleischsuchmaschine ist auch anders als eine Personensuchmaschine. Die Personensuchmaschine ist nichts anders als die normale Suchmaschine im Internet. Der Unterschied liegt nur darin, dass die Personensuchmaschine nur auf der Suche nach Personen bzw. die persönlichen Informationen fokussiert. Das Geschäftsmodell der Personensuchmaschinen basiert darauf, so viel wie möglich die im Internet „frei“ verfügbaren persönlichen Informationen zu sammeln, und für die Suche durch den Kunden nach bestimmten Personen bereitzustellen. ${ }^{28}$ Der Betreiber der Personensuchmaschine hat die personenbezogenen Informationen selbst gesammelt. Der normale Internetnutzer spielt bei der Bereitstellung der persönlichen Informationen der zu suchende Person keine Rolle. Das ist der essentielle Unterschied zwischen Personensuchmaschine und Menschenfleischsuche. Die Rechtssituation über Personensuchmaschine wird in dieser Arbeit nicht diskutiert.

Nach der Erklärung oben wird die Dimension der Menschenfleischsuche im Sinne dieser Arbeit festgestellt. Bevor der rechtliche Aspekt der Menschenfleischsuche analysiert wird, werden im Folgenden erst die Hintergründe für die Entstehung und Entwicklung der Menschenfleischsuche in China diskutiert. Der folgende Teil ist für die Nicht-Chinesen besonders wichtig, um die schnelle Entwicklung der Menschenfleischsuche in China besser zu verstehen, weil dieses Phänomen in vielen Ländern als außergewöhnlich bezeichnet würde. Die Untersuchung nach den Hintergründen ist auch notwendig, um später die Hauptfrage dieser Arbeit zu beantworten, nämlich wie man Menschenfleischsuche regulieren soll.

26 Liu Han, Peking University Law Journal 2011, 870, 872.

27 Mayer-Schönberger, S. 2.

28 Spindler/Nink in Spindler/Schuster, $₫ 28$ BDSG, Rn. 1. 


\section{Die Hintergründe der Entstehung und schnellen Entwicklung der Menschenfleischsuche in China}

Menschenfleischsuche ist ein Phänomen weltweit. Einige Beispiel werden später in der Arbeit erwähnt. Jedoch ist Menschenfleischsuche in China besonders populär. Es werden immer neue Fälle von Menschenfleischsuche in China passieren. Im Januar 2014 wurde eine Reiseführerin wegen Beleidigung eines Touristen von den Internetnutzern durch Menschenfleischsuche gesucht. ${ }^{29}$ Die hohe Häufigkeit der Menschenfleischsuche in China hat ihre Hintergründe.

\section{Technischer Hintergrund}

Die schnelle Entwicklung der Menschenfleischsuche in China liegt erstens daran, dass das Internet in China sehr schnell popularisiert ist, und die Menge der Internetnutzer stark gestiegen ist.Gemäß dem zweiunddreißigsten „statistischen Bericht über die Entwicklungsumstände des Internets in China“, der von CNNIC (China Internet Network Information Center) am 17.07.2013 veröffentlicht wurde, hatte China bis Ende Juni 2013591 Millionen Internetnutzer. ${ }^{30}$ Die Rate der Popularität ist 44.1\%. ${ }^{31}$ Davon benutzen 464 Millionen Internetnutzer ihren Handys, um im Internet zu surfen. Das sind 78.5\% aller Internetnutzer. ${ }^{32}$ Besonders die Popularität des Handys macht es den Internetnutzern möglich, zu jeder Zeit an jedem Ort Nachrichten zu lesen, und an den öffentlichen Diskussionen im Internet teilzunehmen.

Die Internetnutzer von Blogs, soziale Netzwerke und Mikro-Blogs, wo die meisten Menschenfleischsuchen stattfinden, sind je 401 Millionen $^{33}$, 288 Millionen $^{34}$ und 331 Millionen $^{35}$, und sind je $68.0 \%{ }^{36}, 48.8 \%^{37}, 56.0 \%{ }^{38}$ der gesamten Internetnutzer. Die Menge der Internetnutzer garantiert vom Aspekt der Bevölkerungsbasis die Entwicklung der Menschenfleischsuche.

29 He Yucong, Kun ming xin xi gang 07.01.2014, http://news.qq.com/a/20140107/013683. htm (besucht am 04.04.2015)

30 CNNIC, S. 11.

31 CNNIC, S. 11.

32 CNNIC, S. 12.

33 CNNIC, S. 35.

34 CNNIC, S. 37.

35 CNNIC, S. 36.

36 CNNIC, S. 35.

37 CNNIC, S. 37.

38 CNNIC, S. 36. 
Zu betonen ist die Einwirkung des Mikro-Blogs in China. Mikro-Blog funktioniert einfach, hat niedrige Einstiegsvoraussetzungen und ist gleichzeitig hoch interaktiv zwischen den Benutzern. Diese Merkmale des Mikro-Blogs ermöglichen jedem Internetnutzer die gleiche Chance zu haben, ihre Meinung auszudrücken und an einer öffentlichen Diskussion teilzunehmen. Auch die unbedeutende Person kann durch die einzigartige Meinungsäußerung die Aufmerksamkeit der Internetnutzer gewinnen. ${ }^{39}$

Die neue Kommunikationsmöglichkeit wie Mikro-Blog hat neue Wege für die öffentliche Meinungsäußerung geschaffen. Die Atmosphäre der Kommunikation ist gelockert; die Geschwindigkeit der Verbreitung von Informationen ist beschleunigt; das Bewusstsein des normalen Volkes, sich um öffentliche Angelegenheiten zu kümmern, ist verstärkt. ${ }^{40}$ Das Volk ist mutiger geworden, an öffentlichen Diskussionen teilzunehmen. Das ist ein wichtiger Grund der schnellen Entwicklung der Menschenfleischsuche in China.

Nicht zu vernachlässigen ist es aber, dass $36.3 \%$ der Internetnutzer den Bildungshintergrund nur bis Ende der Schule haben, während $11.2 \%$ der Internetnutzer keinen Schulabschluss haben. ${ }^{41}$ Das heißt, fast die Hälfte der Internetvölker hat nur den Bildungshintergrund höchstens bis zur Schule. Deswegen ist es nicht zu erwarten, dass alle Internetnutzer niveauvoll an den Angelegenheiten im Internet teilnehmen können.

\section{Kultureller Hintergrund}

Die online Gesellschaft ist eine Erweiterung der offline Gesellschaft. ${ }^{42}$ Um den Grund der Häufigkeit der Menschenfleischsuche in China zu ermitteln, ist eine Untersuchung der chinesischen offline Kultur notwendig.

\section{a) Schwache Gewichtung vom Schutz der Privatsphäre}

In China ist die kulturelle Tradition, die Privatsphäre der anderen zu respektieren oder zu schützen, schwach ausgeprägt. ${ }^{43}$ Dementsprechend wird im chinesischen Gesellschaftsleben nicht der Begriff "Individuum“ sondern der Begriff „Kollektiv“ betont. Die Nachbarschaft in China als ein Beispiel genommen, ist

39 Xiao Rong, Legal Science Monthly 2012, No. 5, 75, 76.

40 Zhang Zicheng, Academic Exchange 2009, No. 4, 62, 63.

41 CNNIC, S. 16.

42 Vgl. Liang Chen, Internet Law Watch 2008, No. 1, 7ff.

43 Chen Shuyan, Theory Monthly 2011, No. 5, 105, 107; Mei Shaozu, Academic Journal of Suzhou University (Philosophy and Social Science) 2005, 25; Wu Bo, S. 12. 
es die Tradition, dass die Nachbarn einander helfen und sich um einander sorgen. ${ }^{44}$ Die Nachbarschaft ist die sogenannte „Bekannten-Gesellschaft“. Um so eine Gesellschaftsbeziehung zu halten, verzichten die Nachbarn auf Teile ihrer Privatsphäre. Die Verweigerung, die eigene Privatsphäre mit anderen Nachbarn zu teilen, bedeutet auch die Verweigerung, sich in die Nachbargesellschaft zu integrieren. Solche Personen bekamen auch keine Hilfe von den Anderen.

Diese kulturelle Tradition kann man immer noch von den alltäglichen Grußwörtern zwischen den Nachbarn erkennen. Wenn sich die Nachbarn in China begegnen, wird häufig gefragt, ob man gegessen hat, wo kommt man gerade her und wo geht man gleich hin, was eher zur privaten Angelegenheit gehört. Es ist zu sehen, dass es in China eine Tradition ist, um das Leben der anderen zu sorgen.

Mit der Modernisierung des Wohnstils in der Stadt ist die Tradition zwischen den Nachbarn einigermaßen zerstört. Der traditionelle Gedanke, sich um den Mitmensch zu kümmern, ist jedoch in die online-community verlagert, und spiegelt sich in Form von Menschenfleischsuche wieder.

Der kollektive Gedanke besteht auch darin, dass eine Person in China zuerst zu dem Kollektiv, dann zu der Familie und erst zum Schluss zu sich selbst gehört. ${ }^{45}$ Jemand, der die kollektive Moral gebrochen hat, ist unwürdig, Privatsphäre zu haben. Er soll von den Mitmenschen beschimpft oder sogar von dem Kollektiv verbannt werden. ${ }^{46}$ Übrigens soll die Familie als Mittelstufe zwischen dem Kollektiv und dem Individuum für das rechts- und moralwidrige Verhalten ihres Familienangehörigen verantwortlich sein. Dazu gibt es in China ein Sprichwort: „Für die von dem Sohn begangenen Fehler soll der Vater schuld sein." Nach der Logik der chinesischen Philosophie ist der Charakter des Individuums das Ergebnis der Familienerziehung. ${ }^{47}$ Das unschickliche Verhalten eines Familienangehörigen ist der Beweis der gescheiterten Familienerziehung. Das ist der Grund, warum die Informationen der Verwandten der Zielperson während der Menschenfleischsuche auch oft veröffentlicht werden.

Die schwache Gewichtung vom Schutz der Privatsphäre als eine Tradition kann man auch von der Gesetzgebung über Privatsphäre in China erkennen. Vor Inkrafttreten des Delikthaftungsgesetzes am 26.12.2009, gab es im chinesischen Recht kein Begriff über „das Recht auf Privatsphäre“. Die Privatsphäre wurde damals nur im Rahmen von Ehrenschutz geschützt.

44 Xiao Qunzhong, Confucius Studies 2009, No. 4, 17.

45 Vgl. Liu Han, Peking University Law Journal 2011, 870, 878.

46 Liu Han, Peking University Law Journal 2011, 870, 878.

47 Liu Han, Peking University Law Journal 2011, 870, 877f. 


\section{b) Schwaches Rechtsbewusstsein des Volkes}

Für mehrere tausend Jahre hat China immer „rule of man“48 als rechtliche Struktur für den Staat geschaffen. Die Gesetze damals waren eher Straf- und Verwaltungsgesetze. Bei den zwischenmenschlichen Beziehungen spielt die moralische Gerechtigkeit eine größere Rolle. Auf der anderen Seite ist das Rechtsbewusstsein des Volkes relativ schwach ausgeprägt. ${ }^{49}$ Das hat zur Folge, dass bei einem Streit zwischen den Bürgern versucht wird, das Problem „mit Gewalt“ $\mathrm{zu}$ lösen. ${ }^{50}$

Die Menschenfleischsuche wird von den Chinesen häufig benutzt, um die Privatsphäre und das Privatleben der Zielperson, die sich rechts- oder moralwidrig verhalten hat, zu erforschen und offenzulegen, um sie zu bestrafen. ${ }^{51}$ Dahinter steht der Gedanke, dass die Bürger statt durch rechtlichen Weg lieber durch eigene Kraft „Gewalt gegen Gewalt" das rechts- oder moralwidrige Verhalten der Zielperson bestrafen möchten. In diesem Sinne ist Menschenfleischsuche eine Form von Lynchjustiz. ${ }^{52}$ Das lässt auch den Gedanken erkennen, dass die chinesischen Bürger statt Prozessgerechtigkeit eher nach Materialgerechtigkeit streben.

Solche Menschenfleischsuche zeigt weiterhin das schwache Rechtsbewusstsein des chinesischen Volkes in dem Sinne, dass die Rechte der Zielperson völlig vernachlässigt sind. Solche Menschenfleischsuche ist ziemlich ähnlich wie eine chinesische traditionelle beleidigte moralistische Strafe „shame parade“, also "An den Pranger stellen und die Straße entlang Parade machen ${ }^{\text {“ }} .^{53}$ Nicht nur im alten China, sondern auch während der Kulturrevolution war „shame parade“ praktiziert worden, und hatte in dieser Zeit ihren Höhepunkt. Sogar im November 2006 hat die Polizei in Shenzhen für 100 Prostituierten und andere bezüglichen Verdächtigten eine „shame parade“ gemacht. ${ }^{54}$ Über die Rechtswidrigkeit des Verwaltungshandelns der Polizei gab es damals intensive Diskussionen. An diesen Beispielen ist erkennbar, wie groß der Einfluss der chinesischen traditionellen Kultur auf das heutige Verhalten chinesischer Bürgern und sogar Beamten noch ist.

48 Wikipedia: http://en.wikipedia.org/wiki/Rule_of_man (besucht am 04.04.2015).

49 Yin Yijun, S. 17.

50 Vgl. Zhu Juan, Science of Law 2009, No. 1, 47, 53.

51 Yin Yijun, S. 17.

52 Yin Yijun, S. 17.

53 Liu Han, Peking University Law Journal 2011, 870, 878.

54 http://society.people.com.cn/GB/8217/75496/ (besucht am 04.04.2015). 


\section{Politische und soziale Hintergründe}

\section{a) Die Explosion der gebannten Meinungsfreiheit im Internet}

Die Struktur der Politik spielt eine Rolle für die Gestaltung der Internetwelt. ${ }^{55}$ Aufgrund der Unterschiede in der Politik ist die Internetwelt in China und in westlichen Ländern unterschiedlich gestaltet und spielt deswegen in der Gesellschaft auch unterschiedliche Rollen. In westlichen Ländern, spielen traditionelle Medien wie Fernsehen und Zeitung für die Meinungsbildung und Sozialaufsicht die Hauptrolle, obwohl das Internet gut entwickelt ist. Das Internet hat eher die Funktion der Vergnügung.Die traditionellen Grundrechte wie Meinungsfreiheit, Vereinigungs- und Versammlungsfreiheit sowie Demonstrationsfreiheit erfahren durch die chinesischen Gesetze keine ausreichende Durchsetzungsmöglichkeit. In der Praxis ist es auch schwierig, solche Grundrechte tatsächlich zu bewahren. So wird in China z.B., bezüglich der Meinungsfreiheit eine strenge Kontrolle auf Bücher, Zeitungen, Funk, Fernsehen und andere traditionellen Medien ausgeübt. ${ }^{56}$ Ein Überblick auf diesen Kontrollen läuft wie Folgt:

Die zuständige Abteilung im Staatsrat entscheidet über die Menge und Struktur der Publikationsunternehmen, Funkstationen, Fernsehstationen im ganzen Staat. ${ }^{57}$ Nur die juristische Person, die eine für sie verantwortliche (dem Staat zugehörige) Organisation hat, ist qualifiziert, ein Publikationsunternehmen zu gründen. Der Antrag auf Gründung des Publikationsunternehmens muss von der zuständigen Behörde der Provinz und der zuständigen Abteilung des Staatsrates genehmigt werden. ${ }^{58}$ Die für die Publikationsunternehmen verantwortliche Organisation ist verpflichtet, die wichtigen Arbeitspläne zu Themasuchen und Berichten zu überprüfen, die Veröffentlichung wichtiger Berichte, Kommentare, Aufsätze zu überprüfen, bevor eine Publikation durchgeführt wird. Die verantwortliche Organisation soll unmittelbar für die schlimmen Fehler und andere schlimmen Probleme in Bezug auf die Inhalte der Publikation haften. ${ }^{59}$ Für die Publikationsunternehmen wird das Redakteur-Haftung-System verwendet. Der Redakteur muss garantieren, dass

55 Tian Feilong, Internet Law Review 2009, 80, 83.

56 Xiao Rong, Legal Science Monthly 2012, No. 5, 75, 79-80.

$57 \$ 10$ „Verordnung auf der Verwaltung der Publikation“, $\$ 8$ „Verordnung auf Verwaltung des Radios und des Fernsehens“, $\$ 8$ „Verordnung auf Verwaltung der Audio- und Video-Produkte“, $\$ 9$ „Verordnung auf Verwaltung der Veröffentlichung der Zeitschrift“.

$58 \$ 13$ „Verordnung auf Verwaltung der Publikation“.

$59 \$ 8$ "Vorläufige Regelung über die Pflichte der ausrichtenden und verantwortlichen Organisationen der Publikationsunternehmen". 
die Inhalte der veröffentlichten Publikation rechtmäßig sind ${ }^{60}$ Es ist auch die Aufgabe der zuständigen Behörde, die Inhalte und Qualität der Publikation zu überwachen. ${ }^{61}$ Die Publikationsunternehmen haben die Pflicht, das Exemplar der Publikation der zuständigen Behörde vorzuzeigen, um eine Überprüfen zu erleichtern. Sonst besteht für das Unternehmen die Gefahr, dass die Publikationserlaubnis von der Behörde entzogen wird. ${ }^{62}$

Die Rundfunkstationen und die Fernsehstationen können nur von den Behörden der Regierung gegründet werden. Die anderen Unternehmen und natürlichen Personen dürfen keine Rundfunk- oder Fernsehstation gründen. ${ }^{63} \mathrm{Die}$ Rundfunkstationen und die Fernsehstationen sollen ihre Programme vor Ausstrahlung überprüfen lassen. Wiederholt gesendete Programme sollen ebenfalls wiederholt überprüft werden. ${ }^{64}$

Für die Zeitungen und Zeitschriften hat China ein nachträgliches „prüfendLesen-System" entwickelt. ${ }^{65}$ Die Behörde der Publikation und die verantwortliche Organisation für die Zeitungen oder Zeitschriften sollen nach ihrer Publikation die Inhalte und die Qualität überprüfen und bewerten. ${ }^{66}$ Beim prüfenden Lesen gibt es insgesamt 10 Kategorien zu bewerten. 7 Kategorien beziehen sich auf den Inhalt und 3 Kategorie auf die Qualität. ${ }^{67}$ Die gefundenen Ergebnisse des prüfenden Lesens sind ausschlaggebend dafür, ob die Publikationsunternehmen die Jahresprüfung bestehen bzw. ob das Unternehmen weiterhin die Erlaubnis zur Publikation erhält.

Die Meinungsfreiheit des chinesischen Volkes war so lange unter Druck gesetzt, dass das Internet wegen der Popularität, der Interaktivität, des offenen und volksnahen Charakters sofort großes Interesse und Aufmerksamkeit geweckt hat, als es in das Blickfeld des Volkes trat. ${ }^{68}$ Das Volk hat es aufgegeben, die Meinung durch traditionelle Medien auszudrücken und hat sich dem neuen

$60 \$ 24$ „Verordnung auf Verwaltung der Publikation“, $\$ 18$ „Administrative Regelung auf Verwaltung der elektronischen Publikation“, $\$ 24$ „Administrative Regelung auf Verwaltung der Veröffentlichung der Zeitung“, $\$ 24$ „Verordnung auf Verwaltung der Veröffentlichung der Zeitschrift“.

$61 \$ 50$ „Verordnung auf Verwaltung der Publikation“.

$62 \$ \$ 34,67$ „Verordnung auf Verwaltung der Publikation“.

$63 \$ 10$ „Verordnung auf Verwaltung des Radios und des Fernsehens“.

$64 \$ 33$ „Verordnung auf Verwaltung des Radios und des Fernsehens“.

$65 \$ 47$ „Administrative Regelung auf Verwaltung der Veröffentlichung der Zeitung”, $\$ 46$ “Verordnung auf Verwaltung der Veröffentlichung der Zeitschrift".

$66 \$ 2$ „Vorläufige Methode zum „Prüfend-Lesen“ der Zeitung und Zeitschrift”.

$67 \$ 9$ „Vorläufige Methode zum „Prüfend-Lesen“ der Zeitung und Zeitschrift”.

68 Xiao Rong, Legal Science Monthly 2012, No. 5, 75, 80. 
Feld des Internets zugewendet. ${ }^{69}$ Die traditionellen Medien in China verlieren langsam ihren Markt während sich die Internetmedien schnell entwickeln. ${ }^{70} \mathrm{Als}$ neues Feld für Meinungsäußerung füllt das Internet die Lücke der mangelnden Durchsetzbarkeit der Grundrechte. ${ }^{71}$ Menschenfleischsuche ist eben unter diesem Hintergrund entstanden und hat sich schnell verbreitet. ${ }^{72}$ Ich würde sagen, Menschenfleischsuche ist das Ergebnis der Explosion der gebannten Meinungsfreiheit des chinesischen Volkes durch das Internet.

Explosion bedeutet auch nicht oder schlecht kontrollierbar. Genau weil das Volk wegen der strengen Kontrolle der traditionellen Medien für zu lange Zeit unterdrückt war, hat es bei der Meinungsäußerung im relativ freien Internet die Schwierigkeit, sich zu kontrollieren. ${ }^{73}$ Dafür ist die bösartige Seite der Menschenfleischsuche ein guter Beweis.

\section{b) Das Misstrauen der Bürger gegen die Hauptmedien und die Regierung}

Das Misstrauen gegen die Hauptmedien und die Regierung ist ein weiterer Grund der schnellen Entwicklung der Menschenfleischsuche. Es ist nicht schwierig zu verstehen, dass die Hauptmedien wegen der strengen Kontrolle die Stimme der Regierung vertreten. Die niedrige Transparenz des Verhaltens der Regierung führt zu den Misstrauen der Bürger gegen die Regierung. Das Misstrauen gegen die Hauptmedien und der Regierung führt dazu, dass es die Bürger bevorzugen, die Wahrheiten eines Ereignisses eher von den anderen Internetnutzern als von der Regierung zu bekommen. ${ }^{74}$ Die Abhängigkeit der Justiz von der Regierung begründet auch das Misstrauen der Bürger an den Gerichtsverfahren. Dies führt weiter dazu, dass die Internetvölker es vorziehen, ihre eigenen Kräfte einzusetzen, um rechts- bzw. moralwidriges Verhalten zu bestrafen (Menschenfleischsuche als Lynchjustiz), als ein Gerichtsverfahren durchzuführen.

69 Zhang Zuoguo, Western Law Review 2009, No. 6, 7, 9.

70 Xiao Rong, Legal Science Monthly 2012, No. 5, 75, 80.

71 Qi Le, Journal of Inner Mongolia University for Nationalities (Social Sciences) 2009, 104, 105.

72 Qi Le, Journal of Inner Mongolia University for Nationalities (Social Sciences) 2009, 104, 105.

73 Vgl. Zhang Zuoguo, Western Law Review 2009, No. 6, 7, 9.

74 Vgl. Hu Qingfang, Du shu 2008, No. 11, 148ff. 


\section{Psychologischer Hintergrund}

Es ist von den Chinesen allgemein anerkannt und auch seit langem von dem chinesischen Schriftstellern beschrieben oder kritisiert, dass die Chinesen „zha dui“ und „kann re nao“ mögen, welche „sich wegen Geschehnisse spontan sammeln“ und „die Geschehnisse als Show zuschauen“ bedeutet. Für diesen Charakter spielt Neugier der Hauptrolle. Das ist ein wichtiger Grund der zahlreiche Teilnahme an der Menschenfleischsuche.

Das aktive Teilnehmen als Informationseintragender hat jedoch andere psychologische Motivationen Die Entwicklung des Internets ermöglicht jedem Herr seiner Meinung zu sein. Solange die Meinungsäußerung vernünftig ist, wird derjenige leicht von anderen Internetnutzern Zustimmung erhalten. Menschenfleischsuche ist ein Prozess der Gruppenteilnahme. Jeder Beiträger der wertvollen Information wird von den anderen bejaht und gepriesen. Die Anerkennung durch den anderen Internetnutzer ist die höchste Belohnung der Informationseintragenden. Das Gefühl des Erfolgs und dazu entstehende Eitelkeit sowie die wegen der Bejahung der anderen entstandene psychologische Zufriedenheit sind die Motivationen der Informationssammler, um Informationen der Zielperson zu entdecken. ${ }^{75} \mathrm{Zu}$ erwähnen ist es auch, dass es auf mancher Webseite virtuelles Geld als Belohnung für das wertvolle Eintragen gibt.

Für den Kommentator während der Menschenfleischsuche gibt es wieder andere Gedanken. Erstens, die Zielperson hat meistens rechtswidriges oder moralwidriges Verhalten vorgenommen. Der Kommentator kann durch das Kritisieren der Zielperson seinen moralischen Vorzug zeigen, weil er dagegen kein rechtswidriges Verhalten begangen hat. ${ }^{76}$ Zweitens, das anonyme Internet ist mit geringem Risiko der Strafe ein Paradies der Frechheit. ${ }^{77}$ Das alte chinesische Sprichwort „das Gesetz bestraft nicht die ganze Gruppe“ kann dieses Phänomen sehr gut erklären. Wenn eine Person in die Internetwelt eingetreten ist, entsteht bei ihm automatisch ein Gefühl der Sicherheit wegen der Zugehörigkeit in einer Gruppe. Aufgrund dieses Sicherheitsgefühls lässt diese Person ihrem Verhalten freien Lauf, ohne sich um die Gefahr strafbaren Verhaltens zu sorgen. ${ }^{78}$ Dies wird weiterhin zu irrationelles ${ }^{79}$, ungerechtes Missverhalten der Gruppe führen. ${ }^{80}$

75 Shi Bifan, Zhejiang Social Sciences 2011, 34, 35.

76 Shi Bifan, Zhejiang Social Sciences 2011, 34, 35.

77 Liu Peihe/Tian Yining, Contemporary Law Review 2009, No. 3, 127, 128.

78 Liu Peihe/Tian Yining, Contemporary Law Review 2009, No. 3, 127, 128.

79 Zhang Zuoguo, Western Law Review 2009, No. 6, 7, 8.

80 Liu Peihe/Tian Yining, Contemporary Law Review 2009, No. 3, 127, 128; Zhang Zuoguo, Western Law Review 2009, No. 6, 7, 8. 


\section{Die Bewertung der Menschenfleischsuche in China}

In China gibt es von offizieller Seite bisher noch keine deutliche Stellung gegenüber der Menschenfleischsuche, obwohl sie ganz oft und immer wieder geschieht. Um die Rechtsmäßig- oder Rechtswidrigkeit der Menschenfleischsuche zu beurteilen, ist eine genaue Untersuchung ihrer positiven und negativen Seite notwendig.

\section{Die negativen Seiten der Menschenfleischsuche}

\section{a) Menschenfleischsuche hat den Charakter einer Lynchjustiz}

Menschenfleischsuche ist ähnlich wie ein „moralisches Urteil“ von allen teilnehmenden Internetnutzern. Die Zielperson, die moralistisch oder rechtlich negativ bewertet wurde, wird mit der Kritik anderer Bürger konfrontiert. Aber bevor die Zielperson als Schuld beurteilt wird, hat sie keine Chance gehabt, sich zu verteidigen. ${ }^{81}$ Prozessgerechtigkeit wird hier vernachlässigt. ${ }^{82}$

Übrigens wird die Schuld der Zielperson ohne gerichtlichen Prozess beurteilt. Nach der Beurteilung gehen manche Internetnutzer persönlich zur Zielperson, um ihr Verhalten gegenwärtig zu kritisieren, um die Zielperson sogar körperlich zu verletzen, oder durch andere Weise die Zielperson zu bestrafen. Dies widerspricht zum einen der Unschuldsvermutung, wonach die Zielperson bis zum gesetzlichen Beweis ihrer Schuld als unschuldig gelten soll und zum anderen dem staatlichen Strafmonopol, wonach nur die Richter als rechtlich geschulte Fachleute eine Entscheidung über die Schuld der Zielperson treffen können.

Tatsächlich soll, auch wenn die Zielperson moralistisch und rechtlich negativ bewertet ist, die Kritik von den anderen Internetnutzern auf dem Niveau des Meinungsdruckes bleiben. Die Internetnutzer dürfen nicht darüber hinaus durch ihre eigene Kraft die Strafe der Zielperson durchsetzen. ${ }^{83}$

81 Zhang Zhuochen, Science \& Technology Information 2009, 770, 771; siehe auch Qi Le, Journal of Inner Mongolia University for Nationalities (Social Sciences) 2009, 104, 105.

82 Qi Le, Journal of Inner Mongolia University for Nationalities (Social Sciences) 2009, 104, 105; vgl. Zhang Shujun/Du Jin, Journal of Yunnan University Law Edition 2009, No. 4, 66, 68; siehe auch Zhang Zhuochen, Science \& Technology Information 2009, 770, 771.

83 Luo Kun, People’s Court Daily, 12.08.2008; Qi Le, Journal of Inner Mongolia University for Nationalities (Social Sciences) 2009, 104, 105. 


\section{b) Irrationalität und Einseitigkeit}

Menschenfleischsuche führt naturgemäß leicht $\mathrm{zu}$ irrationellem InternetMobbing. ${ }^{84}$

Laut der Theorie der Schweigespirale hängt die Bereitschaft vieler Personen, sich öffentlich zu ihrer Meinung zu bekennen, in bestimmten Fällen von der wahrgenommenen Mehrheitsmeinung ab. ${ }^{85}$ Die meisten Personen haben "Isolationsfurcht", wollen nicht sozial isoliert werden. ${ }^{86}$ Solch ein Gedanke der Internetnutzer führt leicht dazu, dass ihre Meinungen im Internet von der „herrschenden Meinung" abgelenkt und irregeleitet werden.$^{87}$

Obwohl durch die Menschenfleischsuche eine große Menge von Informationen über die Zielperson gesammelt wird, sind die Informationen trotzdem unvollständig. Darüber hinaus spiegeln nicht alle Informationen die wahre Seite der Zielperson wieder. Die unvollständigen Informationen führen leicht zu einem einseitigen Eindruck über die Zielperson, und zwar meistens zu einem schlechten Eindruck. ${ }^{88}$

Außerdem tendiert die Person laut "Selective Exposure Theory“ dazu mit demjenigen zu korrespondieren, der eine ähnliche oder gleiche Meinung hat, um die Gruppenidentifizierung zu verstärken. ${ }^{89}$ Die negative Bewertung zu dem rechts- oder moralwidrigen Verhalten der Zielperson führt leichter zu Gruppenidentifizierung. Dies hat zur Folge, dass die positive Information der Zielperson häufig absichtlich vernachlässigt wird, um die von den meisten Internetnutzern gewollte Bewertung über die Zielperson zu erreichen. ${ }^{90}$

Übrigens ist Menschenfleischsuche schlecht kontrollierbar. Wenn der Veranlasser einmal die Suche eingeleitet hat, kann er die Entwicklung der Suche nicht mehr kontrollieren. Deswegen passiert es häufig, dass die Suche von dem originalen Ziel abweicht, und das Mittel geworden ist, mit dem die Internetnutzer die Privatsphäre unschuldiger Personen aufdecken, um Rache auszuüben,

84 Shi Bifan, Zhejiang Social Sciences 2011, 34, 35; siehe auch Zhang Zuoguo, Western Law Review 2009, No. 6, 7, 8.

85 Wikipedia, http://de.wikipedia.org/wiki/Schweigespirale (besucht am 04.04.2015).

86 Wikipedia, http://de.wikipedia.org/wiki/Schweigespirale (besucht am 04.04.2015).

87 Zhang Shujun/Du Jin, Journal of Yunnan University Law Edition 2009, No. 4, 66, 68; vgl. Qi Le, Journal of Inner Mongolia University for Nationalities (Social Sciences) 2009, 104, 105.

88 Zheng Gencheng, Morality and Civilization 2010, No. 5, $121 \mathrm{ff}$.

89 Zheng Gencheng, Morality and Civilization 2010, No. 5, $121 \mathrm{ff}$.

90 Ma Xudong, Journal of Tianzhong 2011, 38, 39. 
oder andere Art Internetgewalt durchzusetzen oder einfach schlechte Laue abzulassen. ${ }^{91}$

Dies ist in China besonders Problematisch, weil die Meinung herrscht, dass die niedrige Klasse in China zurzeit eine "Halluzination über ihre Macht“ erfährt, ${ }^{92}$ die sie niemals gehabt hat. In dieser Halluzination versinkt der Gedanke über die zu schützenden Rechte der anderen bis zum niedrigsten Niveau. Das Gefühl der Internetnutzer, ihre Macht, die andere Person negativ zu bewerten, auszuüben, übersteigt ihre Rationalität. Die mögliche Verletzung der Rechte der Zielperson wird in der Halluzination nicht mehr berücksichtigt. ${ }^{93}$

\section{c) Die Gefahr falscher Verdächtigung}

Unvermeidlich führt eine Menschenfleischsuche manchmal zum falschen Ergebnis. Statt der gewollten Zielperson kann eine irrelevante schuldlose Person Opfer der Suche werden.

\section{d) Die Gefahr der Informant-Kultur}

Menschenfleischsuche ermutigt die Internetnutzer, die Informationen der Mitmenschen zu verraten. Dies führt weiterhin zur Informant-Kultur, die in Deutschland während der Zeit des Nationalsozialismus und in China während der Zeit der kulturellen Revolution verbreitet war, was in beiden Ländern zu einer Katastrophe geführt hatte. Die Völker mussten in einer unsicheren und Privatsphäre unfreundlichen Gesellschaft leben. ${ }^{94}$

\section{Die positiven Seiten der Menschenfleischsuche}

a) Um Korruption oder anderes rechtswidriges Verhalten von Beamten aufzudecken

Einige positive Wirkungen der Menschenfleischsuche sind mit ihren Motivationen korrespondiert. Zum Beispiel hat die Menschenfleischsuche die Funktion, Korruption oder anderes rechtswidriges Verhalten von Beamten aufzudecken.

91 Li Guoying, Jinling Law Review 2010, 70.

92 Wang Yijun, China Youth Daily, 24.9.2008, http://www.cq.xinhuanet.com/2008-09/24/ content_14479879.htm (besucht am 04.04.2015).

93 Zhou Yuanqing, Law Science Magazine 2011, No. S1, 199, 200.

94 Qi Le, Journal of Inner Mongolia University for Nationalities (Social Sciences) 2009, $104,106$. 
Menschenfleischsuche ist zurzeit ein wichtiges Mittel des chinesischen Volkes geworden, sein demokratisches Aufsichtsrecht wahrzunehmen. ${ }^{95}$

\section{b) Um rechtswidriges Verhalten, insbesondere das Verbrechen der normalen Bürger aufzudecken}

Bei der Funktion, rechtswidriges Verhalten, insbesondere das Verbrechen aufzudecken, ist Menschenfleischsuche wegen der hohen Interaktivität und zahlreicher Teilnehmer ${ }^{96}$ besonders effizient. Häufig sind sie effizienter als die Arbeit der Polizei. Das ist schon durch zahlreiche Fälle bewiesen. Diesbezüglich hat die „Los Angeles Times“ die Menschenfleischsuche sogar als effizienter als die „Internationale kriminalpolizeiliche Organisation-Interpol " bewertet. ${ }^{97}$

Übrigens hat Menschenfleischsuche besonders in China noch die folgenden positiven Seiten.

\section{c) Zur Entwicklung der demokratischen Kultur in China}

Es herrscht die Meinung, dass die Menschenfleischsuche für die Übergangszeit zum demokratischen Staat ein alternativer Mechanismus für die Ausübung der Demokratie des Volks ist. ${ }^{98}$ Die Menschenfleischsuche, besonders die das öffentliche Interesse als Ziel hat, ermöglicht eine rege Teilnahme an den öffentlichen Diskussionen von den Bürgern. Das ist ein wichtiger und notwendiger Schritt für die Entwicklung der demokratischen Kultur. ${ }^{99}$

\section{d) Die Funktion als soziales Sicherheitsventil in China}

Menschenfleischsuche funktioniert als soziales Sicherheitsventil. ${ }^{100}$ Aufgrund der Konflikttheorie von Lewis Coser, ein Soziologe aus den USA, existieren in einer Gesellschaft verschiedene Konflikte. Könnte eine Gesellschaft die Konflikte nicht mehr wirksam behandeln, besonders wenn die Konflikte die Spannungsfähigkeit der Gesellschaftsstruktur überschreiten sollte, stünde die Gesellschaft unter

95 Liu Yijun/Liu Haidong, Journal of Law Application 2010, No. 7, 81, 82.

96 Liu Yijun/Liu Haidong, Journal of Law Application 2010, No. 7, 81, 82.

97 Magnie, Los Angeles Times, 23.11.2008, http://articles.latimes.com/2008/nov/23/ world/fg-scandal23 (besucht am 04.04.2015).

98 Qi Le, Journal of Inner Mongolia University for Nationalities (Social Sciences) 2009, 104, 106; Tian Feilong, Internet Law Review 2009, 80, 82.

99 Qi Le, Journal of Inner Mongolia University for Nationalities (Social Sciences) 2009, 104, 106; siehe auch Tian Feilong, Internet Law Review 2009, 80, 89.

100 Bai Xue, Lan zhou xue kan 2010, No. 9, 111. 
der Gefahr von Zersetzung. Deswegen muss in einer gesunden Gesellschaft ein System bestehen, das wie ein "Sicherheitsventil“ funktioniert, damit der spannende Konflikt zu einer Auslassungsmöglichkeit führen kann, um die Sicherheit des Gesellschaftssystems zu garantieren. ${ }^{101}$

Menschenfleischsuche hat den Völkern einen Weg zur Meinungsäußerung gegeben. Durch diesen Weg können die Bürger die täglich gesammelte Unzufriedenheit gegenüber der Regierung, der Gesellschaft oder anderen Bürgern ausdrücken und ihre schlechte Laune auslassen. ${ }^{102}$ Für die chinesischen Bürger, deren Meinungsfreiheit ziemlich eingeschränkt ist, ist die Menschenfleischsuche als Sicherheitsventil besonders praktisch.

\section{Die Bewertung der Menschenfleischsuche}

Menschenfleischsuche hat ihre negative und positive Seite. Dies führt dazu, dass Menschenfleischsuche nicht allgemein verneint oder bejaht werden kann. ${ }^{103} \mathrm{Als}$ Erstes müssen die Motivationen der Menschenfleischsuche unterschieden werden. Offensichtlich rechtswidrig ist die Menschenfleischsuche mit der Motivation, um Rache auszuüben. ${ }^{104}$

Hinter der Menschenfleischsuche mit anderen Motivationen stehen mehr oder weniger das öffentliches Interesse oder die schutzwürdigen Rechte und Freiheiten des Äußernden. Obwohl oben genannte negative Seiten bestehen, sind alle Nachteile Gefahren, die nicht unbedingt passieren würden. Man kann Menschenfleischsuche nicht wegen Gefährlichkeit völlig verbieten, wenn es Möglichkeit gibt, die Gefahr zu vermeiden. Bestünde diese Möglichkeit, wäre ein völliges Verbot der Menschenfleischsuche unverhältnismäßig. In dieser Arbeit wird genau diese Möglichkeit untersucht. Nach meiner Meinung ist es möglich, durch Regulierung die Rechtmäßigkeit der Menschenfleischsuche zu garantieren oder mindestens deren Rechtswidrigkeit zu verringern.

Übrigens dürfen die Bürger ihre Rechte und Freiheiten ausüben, solange sie nicht vom Gesetz verboten sind. Menschenfleischsuche besteht aus Meinungsäußerungen der Internetnutzer. Ob diese Äußerungen zu Rechtsverletzungen führen, muss in jedem einzelnen Fall geprüft werden. Eine Interessenabwägung ist für viele Fälle häufig notwendig. Das heißt, Menschenfleischsuche bringt

101 Bai Xue, Lan zhou xue kan 2010, No. 9, 111.

102 Xue Xia, China Youth Study 2009, No. 1, 92, 93.

103 Vgl. Su Zhe, Hebei Law Science 2012, No. 2, 26, 27.

104 Siehe oben unter $\$ 1$ III 1 e). 
nicht unbedingt Rechtsverletzungen mit sich. Ein generelles Verbot der Menschenfleischsuche ist daher in China nicht vorgesehen.

\section{Menschenfleischsuche als ein soziales Phänomen weltweit}

Im Sinne der umfangreichen Teilnahme und der Häufigkeit ist die Menschenfleischsuche eine chinesische Besonderheit. ${ }^{105}$ Jedoch passiert Menschenfleischsuche als ein soziales Phänomen überall auf der Welt. Dazu gehört natürlich auch Deutschland.

\section{Ein paar Beispiele der Menschenfleischsuche weltweit}

Im März 2008 wurde es in einem Video aufgenommen, dass ein in Irak stationierter USA-Marine-Soldat einen Welpen von einem Kliff hinuntergeworfen hat. Nach der Veröffentlichung dieses Videos wurde das Verhalten des Soldaten viel kritisiert. ${ }^{106}$ Durch Menschenfleischsuche haben die Internetnutzer zum Schluss festgestellt, dass der Soldat Lance Corporal David Motari ist. Die persönlichen Informationen von Motari wurden dann veröffentlicht. Dazu gehörten seine Telefonnummer und sogar ein Bild von seinem Auto. ${ }^{107}$ Motari wurde zum Schluss von der Marine entlassen und hatte eine „non-judicial“ Strafe bekommen. ${ }^{108}$

Ein anderes Beispiel stammt von Patrick Moberg aus New York City, der eine Webseite erstellt hat. Er wollte eine Frau, mit der er in der U-Bahn netten Augenkontakt aber keine Chance zum Gespräch gehabt hatte, wiederfinden. Er hat auf der Webseite ein selbst gezeichnetes Bild von der Frau publiziert. Mit der Hilfe der Internetnutzer wurde die Frau gefunden. Die beide waren in Kontakt geblieben und haben zum Schluss eine Beziehung begründet. ${ }^{109}$

Ein sehr bekanntes Beispiel passierte im Juni 2005 in Seoul. Ein Mädchen war mit seinem Hund in der U-Bahn unterwegs. Der Hund hat seine Notdurft

105 Zhang Shujun/Du Jin, Journal of Yunnan University Law Edition 2009, No. 4, 66, 67.

106 Bild, http://www.bild.de/news/vermischtes/hund/harte-strafen-fuer-us-soldaten4821120.bild.html (besucht am 04.04.2015).

107 Vgl. Wu Zheng, Xin hua wang 06.03.2008, http://news.xinhuanet.com/news center/2008-03/06/content_7720461.htm (besucht am 04.04.2015); Zhang Shujun/ Du Jin, Journal of Yunnan University Law Edition 2009, No. 4, 66, 67.

108 Wikipedia, http://en.wikipedia.org/wiki/List_of_Internet_phenomena (besucht am 04.04.2015).

109 Vgl. Leonard, http://www.telegraph.co.uk/news/uknews/1568941/Love-at-first-siteon-the-New-York-subway.html (besucht am 04.04.2015). 
verrichtet. Ein alter Mann hatte das Mädchen aufgefordert, den Kot zu beseitigen. Sie hat es nicht gemacht sondern den alten Mann beschimpft. Die Situation wurde aufgenommen und im Internet veröffentlicht. Dieses Video hat schnell Aufmerksamkeit der anderen Internetnutzer geweckt. Das Mädchen wurde im Internet als „Hund-Scheiße-Mädchen“ bezeichnet. Die persönlichen Informationen von ihr und ihren Freunden und ihrer Familie wurden veröffentlicht. Die Bilder von ihr und ihrem Hund wurden auf den Portal-Webseiten in Korea schnell verbreitet. Die nationalen Medien haben danach auch über den Skandal berichtet. Das Mädchen ist wegen dieses Skandals und der Berichte über sie zwingend von ihrer Universität weggezogen. ${ }^{110}$ Nach diesem Ereignis gab es intensive Diskussion über Internet-Mobbing in Südkorea. Um dieses Mobbing zu beschränken hat es Südkorea im Jahr 2009 durch das Gesetz „Real-NameSystem“ im Internet erlassen. ${ }^{111}$ Die Diskussion über „Real-Name-System“ wird später in der Arbeit durchgeführt.

Die Beispiele von Menschenfleischsuche weltweit sind unzählbar. Unter der Kategorie „Internet vigilantism“ hat Wikipedia zahlreiche Beispiele über Menschenfleischsuche gelistet. Technisch gesagt, solange es Internet gibt, kann Menschenfleischsuche passieren. Außerdem gibt es für das Passieren der Menschenfleischsuche allgemein psychologische Gründe.

\section{Menschenfleischsuche und ähnliches Phänomen in Deutschland}

Das Internetforum als die technische Voraussetzung der Menschenfleischsuche hat sich nach der Erfindung des Usenet im Jahr $1979^{112}$ erst in den 1980er-Jahren langsam entwickelt. ${ }^{113}$ Die erste Menschenfleischsuche passierte im Jahr 2005. Aber im Jahr 1970 hat das Land Hessen schon ein Landesdatenschutzgesetz, das erste allgemeine Datenschutzgesetz der Welt, erlassen. ${ }^{114}$ Das bedeutet, schon 35 Jahre vor der tatsächlichen Menschenfleischsuche und sogar mindestens 10 Jahre vor der Möglichkeit der Menschenfleischsuche gab es in Deutschland die Gedanken und die Gesetze, persönliche Daten zu schützen. Dies hat zur Folge, dass die Deutschen mit dem Phänomen der Menschenfleischsuche vorsichtiger sind, weil sie Datenschutzprobleme befürchten.

110 Wikipedia, http://en.wikipedia.org/wiki/Internet_vigilantism (besucht am 04.04.2015).

111 Wikipedia, http://en.wikipedia.org/wiki/Real-name_system (besucht am 04.04.2015).

112 Wikipedia, http://de.wikipedia.org/wiki/Usenet (besucht am 04.04.2015).

113 Wikipedia, http://de.wikipedia.org/wiki/Internetforum (besucht am 04.04.2015).

114 Gola/Schomerus, in Gola/Schomerus, Einleitung, Rn. 1. 
Trotzdem gibt es ein paar Beispiele in Deutschland, die gleich oder ähnlich wie die Menschenfleischsuche in China sind.

\section{a) Private Facebook-Jagd nach einem unbekannten Sextäter-Menschenfleischsuche in Deutschland}

Im Oktober 2013 hatte die Polizei von Frankfurt am Main eine öffentliche Fahndung nach einem Sexualstraftäter durchgeführt. Dabei war ein Phantombild des Verdächtigten publiziert. ${ }^{115}$ Die Fahndung wurde aber von einem Internetnutzer auf Facebook weitergeleitet, und zwar nicht im Name der Polizei, sondern in seinem eigenen Namen. Dieser private Internetnutzer hat mit dem Namen „Auf der Jagt ${ }^{116}$ nach dem Vergewaltiger" ein Facebook-Profil mit der Fahndung und dem Phantombild zusammengestellt und die anderen Internetnutzer gebeten, Hinweise auf den Verdächtigten einzutragen. Dieses Profil hatte in kurzer Zeit mehr als 12,000 "Gefällt mir“ bekommen. Außer den Schimpfwörtern gegen den Straftäter gab es auch viele Hinweise auf den vermutlich Verdächtigten. Dazu gehörten ein von einem Internetnutzer fotografiertes Foto eines Fußgängers, der wie der Verdächtigte aussah, die vermutliche Adresse der Verdächtigten, die Merkmale des Autos der Verdächtigten usw. ${ }^{117}$ Diese Suche wurde als moderne Hexenjagd bezeichnet. ${ }^{118}$

\section{b) Aufruf zum Lynchmord gegen einen 17-jährigen Verdächtigten auf Facebook - Menschenfleischsuche ähnliches Phänomen}

Eben auf Facebook gab es ein anderes Ereignis, das vom Sachverhalt aus ähnlich aussieht aber rechtlich völlig anders berücksichtigt werden soll.

Im März 2012 wurde ein 18-Jähriger wegen Lynchjustiz auf Facebook für zwei Wochen Jugendarrest verurteilt. Nach dem Mord an einem elfährigen Mädchen hatte der 18-Jähriger im März 2012 auf Facebook zum Lynchmord an

115 Phsc/jaar, hr-online.de, 30.10.2013, http://www.hr-online.de/website/rubriken/nachrichten/indexhessen34938.jsp?rubrik=36082\&key=standard_document_49977267 (besucht am 04.04.2015).

116 Originaler Fehler der Rechtschreibung von den Facebook-Profilen.

117 Wotu: hr-online.de, 06.11.2013, http://www.hr-online.de/website/rubriken/nachrichten/indexhessen34938.jsp?rubrik=36082\&key=standard_document_50022359 (besucht am 04.04.2015).

118 http://www.hr-online.de/website/rubriken/nachrichten/indexhessen34938. jsp?key=standard_document_50022359\&jmpage $=1$ \&type $=v \&$ rubrik $=36082 \& j \mathrm{~m}=$ 2\&mediakey=fs/hessenschau/2013_11/131105193846_hs_facebook_16180 (besucht am 04.04.2015). 
einem 17-Jährigen aufgerufen, der irrtümlich in Verdacht geraten war. ${ }^{119}$ Dies hatte zur Folge, dass ständige Proteste und Demonstrationen vor der Haustür des 17-jährigen Verdächtigen stattfanden.Wiedereinführung der Todesstrafe gegen ihn und Erstürmung seiner Wohnung war bei der Demonstration gefordert. ${ }^{120}$

Dieses Ereignis ist anders als die typische Menschenfleischsuche in dieser Arbeit. In diesem Ereignis hat keine Suche stattgefunden. Der 18-jährige Veranlasser hat am Anfang die Zielperson schon mit Namensnennung festgestellt. Der weitere Unterschied liegt darin, dass der Veranlasser beim Erstellen der Facebook-Seite ein klares Ziel gehabt hat, nämlich mit der Kraft des Volkes den 17-Jährigen zu töten. Dieser Unterschied hat zur Folge, dass dieser Fall als Anstiftung zum Totschlag oder Mord strafrechtlich berücksichtigt werden könnte. ${ }^{121}$

\section{c) Die anderen Menschenfleischsuche ähnlichen Ereignisse in Deutschland}

Es gibt in Deutschland häufig Ereignisse, bei denen man die bezügliche Person identifizieren möchte, wie z.B. der unbekannte Fußgänger, der beim Raubüberfall einem alten Mann geholfen hat; ${ }^{122}$ oder ein Bundeswehr-Soldat, der in einem im Internet hochgeladenem Video beim Training auf Befehl des Vorgesetzten ständig "Motherfucker" geschrien hat; ${ }^{123}$ oder die Bundeswehr-Soldaten, die sich in Afghanistan mit einem Totenschädel fotografiert haben; ${ }^{124}$ oder der in einem Autounfall

119 Hessenschau, 05.11.2013, http://www.hr-online.de/website/rubriken/nachrichten/indexhessen34938.jsp? rubrik=36082\&xtmc=Facebook-Jagd\%20auf\%20 Sex-T\%FDter\&type $=$ d\&xtcr=1\&key=standard_document_50022359 (besucht am 04.04.2015).

120 Wiegand, sueddeutsche.de, 07.03.2012, http://www.sueddeutsche.de/leben/protest-ge gen-jugendlichen-sexualstraftaeter-menschenauflauf-fordert-todesstrafe-1.1302098 (besucht am 04.04.2015).

121 Ostendorf/Frahm/Doege, NStZ 2012, 529, 533.

122 Augsburger Allgemeine, 27.09.2013, http://www.augsburger-allgemeine.de/bayern/ Ueberfall-verhindert-Wer-ist-der-unbekannte-Helfer-id27168107.html (besucht am 04.04.2015).

123 Arnsperger, Stern.de, 13.04.2007, http://www.stern.de/politik/deutschland/bundes wehr-feuern-und-motherfucker-bruellen-586855.html (besucht am 04.04.2015).

124 Stern.de, 26.10.2006, http://www.stern.de/politik/deutschland/afghanistan-neueskandalfotos-aufgetaucht-574968.html (besucht am 04.04.2015), Ler/dpa/ddp, Spiegel online, 31.10.2006, http://www.spiegel.de/politik/deutschland/bundeswehrerste-gestaendnisse-im-skandal-um-totenschaedel-fotos-a-445561.html (besucht am 04.04.2015). 
verletzte und in Koma geratene Unbekannte, deren Familie von der Polizei durch das Internet mit Hilfe der anderen Internetnutzer gefunden werden soll. ${ }^{125}$

Diese Suchen sind aber entweder von den Medien oder von der Polizei, nicht von privaten Personen veranlasst. Der Vorgang der Suche ist meistens von der zuständigen Behörde oder von den Medien offline durchgeführt. Die Teilnahme der Internetnutzer wird nicht öffentlich gezeigt, sondern durch einseitigen Kontakt zwischen dem Internetnutzer und den Medien oder der Polizei oder der zuständigen Behörde verwirklicht.

Solche Suche ist nicht die Menschenfleischsuche in dieser Arbeit, kann aber als Vorbild der zukünftigen Menschenfleischsuche berücksichtigt werden.

125 Tismer, TLZ, 26.11.2013, http://eichsfeld.tlz.de/web/lokal/leben/blaulicht/deta il/-/specific/Leinefelde-Unfallopfer-durch-Engagement-und-Internetidentifiziert-577877368 (besucht am 04.04.2015). 


\section{$\$ 2$ Menschenfleischsuche als ein rechtliches Phänomen}

\section{Die Rechtsprobleme während Menschenfleischsuche}

\section{Die Rechtsprobleme allgemein}

Die erst zu diskutierende Frage ist, ob Menschenfleischsuche allgemein rechtswidrig ist. Die Einstellung des chinesischen und deutschen Rechts zur Menschenfleischsuche ist für die weitere Diskussion entscheidend. Um diese Frage zu beantworten, werden die zu schützenden Rechte der Zielperson und die Freiheit und zu schützenden Rechte der Internetnutzer abgewogen.

Menschenfleischsuche als ein Geschöpf des Internet-Zeitalters ist eine Herausforderung für alle Länder der Welt. Durch den Rechtsvergleich zwischen China und Deutschland versucht diese Arbeit einen Gedankengang zur Regulierung der Menschenfleischsuche allgemein bereitzustellen.

\section{Die Rechtsprobleme bezüglich der Veranlasser}

An den zahlreichen Beispielen von Menschenfleischsuche in China, Deutschland und anderen Ländern kann man sehen, dass Menschenfleischsuche zurzeit nicht hundert Prozent „schick“ laufen kann. Das bedeutet, wegen verschiedenen Niveaus und verschiedener Denkweise der Internetnutzer tauchen während der Menschenfleischsuche unvermeidlich böswillige Beiträge auf, die die Rechte der Zielperson verletzen könnten. Bei dem Einleiten der Menschenfleischsuche hat der Veranlasser die Zielperson tatsächlich in eine gefährliche Situation gebracht. $\mathrm{Ob}$ und wie er für die dadurch entstehende Rechtsverletzung haften soll, ist eine Frage dieser Arbeit.

Bei dem Anschalten der Menschenfleischsuche veröffentlicht der Veranlasser häufig Bild, Video oder persönliche Informationen von der Zielperson. Ob und wann eine Rechtsverletzung gegen die Zielperson begründet ist, wird in dieser Arbeit diskutiert.

\section{Die Rechtsprobleme bezüglich der Informationssammler}

Der Informationssammler hat die schon veröffentlichten Informationen der Zielperson gesammelt und für die weitere Menschenfleischsuche zusammengestellt. Ob man die einmal veröffentlichte persönliche Information für beliebige Zwecke verwenden kann, ist eine Frage dieser Arbeit. 
Während der Menschenfleischsuche passiert es auch häufig, dass der Internetnutzer die nur im begrenzten Kreis veröffentlichte Information auf einem größeren Kreis verbreitet. Diese Information wird als nicht veröffentlicht angesehen. Solche Internetnutzer werden als Informationsberichter behandelt.

\section{Die Rechtsprobleme bezüglich der Informationsberichter}

Der Informationsberichter hat die persönlichen Informationen der Zielperson ohne ihre Einwilligung zum ersten Mal im Internet veröffentlicht oder die begrenzt veröffentlichten Informationen einem breiteren Kreis zugänglich gemacht. Für solche Veröffentlichung oder Verbreiterung besteht die Gefahr, in die Privatsphäre der Zielperson einzubrechen oder ihr Recht auf informationelle Selbstbestimmung zu verletzen. Für den Fall der Veröffentlichung bzw. der Verbreitung des Bildes der Zielperson besteht auch die Gefahr vom Verletzen ihres Rechts am eigenen Bild. Unter welchen Voraussetzungen diese Rechtsverletzungen begründet werden, wird in dieser Arbeit diskutiert.

\section{Die Rechtsprobleme bezüglich der ICP und ISP}

ISP ist der Oberbegriff für alle Dienstanbieter, die technische und inhaltliche Leistungen anbieten. ${ }^{126}$ In dieser Arbeit wird der Provider, der den Inhalt als Dienst anbietet, als „Internet Content Provider" (ICP) bezeichnet, um mit anderen ISP zu unterscheiden. Die Bedeutung des ISP wird sich in dieser Arbeit auch spezifisch auf die Provider richten, die durch ihren Dienst eine Kommunikationsplattform in Form von Internetforen, Blogs, Sozialnetzwerken für die Menschenfleischsuche anbieten. Bei Bedarf wird der ISP ausnahmsweise auch als Oberbegriff von allen Dienstanbietern verwendet.

Die Inhalte, die auf der Webseite des ICP stehen, sind entweder von ihm selbst editiert oder von den Internetnutzern eingetragen, aber erst nach seiner Überprüfung zur Veröffentlichung freigeschaltet. Der ICP spielt eine Rolle vergleichbar mit den traditionellen Medien. Er soll Kenntnis haben, welche Informationen auf ihrer Webseite stehen, und prinzipiell für die rechtsverletzenden Inhalte auf seiner Webseite verantwortlich sein. Problematisch ist es, dass ein Provider heutzutage häufig mehrere Funktionen hat. Das heißt, ein Provider kann gleichzeitig ICP und ISP sein. Es ist die Aufgabe dieser Arbeit, den ICP durch sein Verhalten zu identifizieren.

Der ISP in dieser Arbeit bietet den Internetnutzern eine Plattform für Menschenfleischsuche, um ihr Eintragen bzw. Diskussion im Internet zu ermöglichen.

126 Wikipedia,http://de.wikipedia.org/wiki/Internetdienstanbieter (besucht am 04.04.2015). 
Die Eintragungen werden gespeichert und auf Bedarf des Eintragenden völlig oder teilweise veröffentlicht und für den anderen Internetnutzer abrufbar gemacht. ${ }^{127}$ Der Dienst des ISP ist eine Voraussetzung, dass Menschenfleischsuche überhaupt passieren kann. Dies könnte weiterhin dazu führen, dass der ISP wegen des Anbietens der Plattform für die während Menschenfleischsuche passierten Rechtsverletzungen haften sollte. Ob eine Haftung begründet wird und was für eine Haftung es sein soll, wird in dieser Arbeit diskutiert.

\section{Die Rechtsprobleme bezüglich der Zielperson}

Während der Menschenfleischsuche werden die persönlichen Informationen und die Bilder der Zielperson ohne ihre Einwilligung veröffentlicht. Es besteht die Gefahr, dadurch ihr Recht am eigenen Bild, das Recht auf informationelle Selbstbestimmung und das Recht auf Privatsphäre zu verletzen.

Im Fall der begründeten Rechtsverletzung werden die Schutzmöglichkeiten in dieser Arbeit diskutiert. Die Internetnutzer, der ICP und der ISP sind die möglichen Rechtsverletzer. Wie kann die Zielperson am schnellsten die laufenden Rechtsverletzungen aufhalten, welche Ansprüche hat die Zielperson jeweils gegen die Rechtsverletzer, wie kann die Zielperson am besten vor weiteren Rechtsverletzungen geschützt werden - dies sind die zu beantwortenden Fragen. Besonders zu betonen ist es, dass es wegen der Anonymität und Pseudonymität im Internet für die Zielperson häufig schwierig ist, den Internetnutzer als unmittelbaren Rechtsverletzer zu verfolgen ${ }^{128}$, während ein Anspruch gegen den ISP viel einfacher wäre. Das Verhältnis zwischen ISP und Internetnutzer als mögliche Rechtsverletzer einschließlich einer etwaigen Regulierung des Verhältnisses soll eine weitere Aufgabe dieser Arbeit sein.

Nicht jede Veröffentlichung persönlicher Informationen der Zielperson ist rechtswidrig. In manchen Fällen muss die Zielperson die Veröffentlichung ihrer Information dulden. Dies ist besonders der Fall, wenn die Zielperson prominent ist oder einen besonderen Beruf hat. Diese Sonderfälle werden auch in der Arbeit diskutiert.

\section{Die Rechtsprobleme bezüglich der Kommentatoren}

Der Kommentator hat während der Menschenfleischsuche die Zielperson und ihr Verhalten kommentiert. Als Meinungsäußerung gehört das Kommentieren zu der Meinungsfreiheit der Internetnutzer, während die Ehre der Zielperson

127 Vgl. Wilmer, NJW 2008, 1845.

128 Gräbig, MMR 2011, 504. 
durch ungeeignete Kommentare verletzt werden kann. Die Grenze zwischen Meinungsfreiheit und Ehrverletzung wird erklärt.

\section{Die Wichtigkeit der wissenschaftlichen Diskussion über Menschenfleischsuche}

Das Hauptverhalten während einer Menschenfleischsuche ist, die persönlichen Informationen der Zielperson ohne ihre Einwilligung zu veröffentlichen. Dementsprechend besteht die Notwendigkeit, um Menschenfleischsuche zu regulieren, da die Verbreitung der Informationen im Internet-Zeitalter einige Besonderheiten aufweist, wodurch die Rechte der betroffenen Person schwerer verletzt werden könnten. Solche Besonderheiten sind z.B.:

\section{Geographisch unkontrollierbare Verbreitung ${ }^{129} \mathrm{der}$ Information im Internet}

Anders als Druckerzeugnis mit begrenzter Auflage werden die online gestellten Inhalte, die ohne Abruf-Beschränkungen veröffentlicht sind, sofort weltweit abrufbar sein. ${ }^{130}$ Durch einfaches Kopieren und Verlinken werden die Inhalte schnell verbreitet. ${ }^{131}$ Dies ermöglicht im Vergleich mit Offline-Publikation einen größeren potenziellen Adressatenkreis. ${ }^{132}$

Falls die veröffentlichten Inhalte rechtswidrig oder das Recht des Rechtsinhabers schutzwürdiger wäre, entsteht dementsprechend auch größerer Schaden für den Rechtsinhaber.

\section{Zeitlich unkontrollierbare Verbreitung ${ }^{133}$ der Information im Internet}

Die zeitlich unkontrollierbare Verbreitung der Online-Publikation stellt sich in drei Aspekte dar. Erstens können die Internetnutzer die Inhalte, die einmal im Internet veröffentlicht sind, anders als die live Fernsehen- und Radiosendung, jederzeit unbeschränkt wiederholt abrufen und lesen. Zweitens können die im Internet eingestellten Informationen „für einen langen oder

129 Härting, CR 2009, 21, 23; Ballhausen/Roggenkamp, K\&R 2008, 403, 404.

130 Gounalakis/Klein, NJW 2010, 566, 567.

131 Vgl. Heckmann, NJW 2012, 2631, 2634.

132 Gounalakis/Klein, NJW 2010, 566, 567.

133 Härting, CR 2009, 21, 23; Ballhausen/Roggenkamp, K\&R 2008, 403, 404. 
gar unbegrenzten Zeitraum bereitgehalten werden“. ${ }^{134}$ Das ist die sogenannte Dauerhaftigkeit der Internet-Publikation („Zeitfaktor“) ${ }^{135}$ Drittens können die original veröffentlichten Inhalte über Online-Archive immer noch eingesehen werden, obwohl sie gelöscht sind. ${ }^{136}$ Im Fall einer Rechtsverletzung hat dieses Merkmal die gleiche Wirkung wie die geographisch unkontrollierbare Verbreitung.

\section{Unkontrollierbare Vermehrung der Information im Internet}

Nachdem die Information von dem Internetnutzer im Internet eingetragen ist, kann er nicht mehr so über sie verfügen, als ob sie auf seinem eigenen Computer gespeichert wären. ${ }^{137}$ Diese Information wird weiterhin von den Interessierten auf andere Webseiten übertragen oder durch Verlinken weitergeleitet. Die Änderung der originalen Information wäre unter bestimmte Voraussetzung noch möglich. Aber die entsprechende Aktualisierung der kopierten und tief verlinkten Information wird nicht mehr garantiert. Vernachlässigen die Interessierten die Aktualisierung der kopierten oder verlinkten Information, könnten neue Rechtsverletzungen dadurch begründet werden.

Übrigens ist es auch schwierig, die im Internet unkontrollierbar vermehrte Information vollständig zu löschen. Besonders für die Information, die zu einer erheblichen Kontroverse führt und dadurch das Interesse der Internetnutzer weckt, ${ }^{138}$ muss man damit rechnen, obwohl die originale Information durch einen Gerichtsprozess erfolgreich gelöscht ist, dass in irgendeiner Ecke im Internet immer noch eine Kopie existiert, die den Rechtsschutz erschwert oder gar unmöglich macht. ${ }^{139}$

\section{Bildung persönlicher Profile und die Prangerwirkung durch Menschenfleischsuche}

Die Diskussion über Menschenfleischsuche ist auch deswegen wichtig, weil die während der Menschenfleischsuche offengelegten Informationen zu einem persönlichen Profil führen, das potenziell Prangerwirkung hat.

\footnotetext{
134 Grevel/Schärdel, MMR 2008, 644, 648.

135 Härting, CR 2009, 21, 23; Ballhausen/Roggenkamp, K\&R 2008, 403, 404.

136 Gounalakis/Klein, NJW 2010, 566, 567.

137 Wilmer, NJW 2008, 1845.

138 Ladeur/Gostomzyk, NJW 2012, 710, 713.

139 Ladeur/Gostomzyk, NJW 2012, 710, 713.
} 
Durch die Zusammenstellung der persönlichen Informationen der Zielperson während der Menschenfleischsuche kann ein persönliches Profil über die Zielperson leicht erstellt werden. ${ }^{140}$ Die zusammengestellten Informationen werden von irgendeinem Internetnutzer, der daran Interesse hat ${ }^{141}$, leicht aufgefunden. ${ }^{142}$ Dies ermöglicht dem Internetnutzer „deutlich konzentrierter und dadurch auch schneller, Informationen über eine bestimmte Person zu erhalten "143. Wenn die Informationen über eine Person im Internet durch einfache Eingabe des Namens in einer Suchmaschine gefunden werden können, könnte eine Vertiefung des Eingriffs in das Persönlichkeitsrecht des Betroffenen begründet werden. ${ }^{144}$

Die oben genannten Merkmale haben im Jahre 2001 schon die Aufmerksamkeit des BVerfG gewonnen ${ }^{145}$ und werden als Prangerwirkung bezeichnet. Nach der Auffassung des BVerfG lag die „Prangerwirkung“ darin, dass die im Internet veröffentlichten Informationen „für eine unbestimmte und grundsätzlich unbegrenzte Öffentlichkeit verfügbar" waren, und die Nutzbarkeit dieser Informationen durch Suchdienst erleichtert wurde. ${ }^{146}$ Im Fall der Menschenfleischsuche wurde das Auffinden der Information der Zielperson aus großen Datenmassen erleichtert bzw. beschleunigt ${ }^{147}$; und es erlaubt auch demjenigen die Information in kurzer Zeit zu finden, der daran kein unmittelbares oder gar kein Informationsinteresse hat, sondern nur wegen reiner Neugier recherchiert. ${ }^{148}$

\section{Menschenfleischsuche bietet ein typisches Beispiel der Rechtsverletzung durch den Internetnutzer}

Die Wichtigkeit der Diskussion über Menschenfleischsuche liegt weiterhin darin, dass sie ein typisches Beispiel von durch den Internetnutzer begangener Rechtsverletzung zeigt.

140 Vgl. BVerfG, MMR 2002, 89, 90.

141 Härting, CR 2009, 21, 23.

142 Härting, CR 2009, 21, 23; Ballhausen/Roggenkamp, K\&R 2008, 403, 404; Vgl. BVerfG, MMR 2002, 89, 90.

143 Seidel/Nink, CR 2009, 666.

144 Härting, CR 2009, 21, 23.

145 BVerfG, MMR 2002, 89.

146 BVerfG, MMR 2002, 89, 90.

147 BVerfG, MMR 2002, 89, 90.

148 Härting, CR 2009, 21, 23; siehe auch Gounalakis/Klein, NJW 2010, 566, 567. 
Im Zeitalter von „Web 2.0“ ist der Internetnutzer die wichtigste Kraft der Online-Publikation geworden. Die abrufbaren Inhalte im Internet stammen nicht mehr nur von zentralen Content-Anbietern, sondern vorrangig von den Nutzern des Internet. ${ }^{149}$ Dies erhöht das Risiko von Rechtsverletzungen durch den Internetnutzer. Übrigens sind die Äußerungen von den Internetnutzern im Internet durch ihre Anonymität geprägt. ${ }^{150}$ Es ist häufig unklar, wer tatsächlich die Rechtsverletzung begangen hat. ${ }^{151}$ Die Haftung der Internetnutzer und die Durchsetzung der Haftung sind deswegen wichtige Themen.

Durch die Diskussion in dieser Arbeit wird auch versucht, die Frage zu beantworten, wie die durch Internetnutzer begangenen Rechtsverletzungen allgemein behandelt werden sollen.

\section{Die Struktur dieser Arbeit}

Erstens werden die zu schützenden Rechte der Zielperson während der Menschenfleischsuche nach chinesischem und deutschem Recht erforscht. Der Schutzbereich dieser Rechte in den beiden Ländern wird erklärt.

Danach werden die möglichen Rechtsverletzungen von Internetnutzern, ICP und ISP während der Menschenfleischsuche je nach chinesischem und deutschem Rechtssystem diskutiert. Die unterschiedlichen rechtlichen Stellungen gegen das Verhalten von Internetnutzern, ICP und ISP bei der Menschenfleischsuche in beiden Ländern werden erläutert.

Nachdem einige Rechtsverletzungen begründet sind, werden die Möglichkeiten zum Schutz der Rechte der Zielperson diskutiert. Dazu gehören die Ansprüche gegen den rechtsverletzenden Internetnutzer, den ICP und den ISP sowie andere Maßnahmen, die zur Regulierung der Menschenfleischsuche dienen.

Zwischendurch werden die Vergleiche zwischen China und Deutschland durchgeführt.

149 Wilmer, NJW 2008, 1845.

150 Gounalakis/Klein, NJW 2010, 566, 567.

151 Heckmann, NJW 2012, 2631, 2634. 



\section{Kapitel. Die zu schützenden Rechte der Zielperson während der Menschenfleischsuche}

Während der Menschenfleischsuche besteht die Gefahr der Verletzung von vier Rechten der Zielperson. Im Fall der Veröffentlichung ihres Bildes ohne ihre Einwilligung könnte ihr Recht am eigenen Bild das Schutzobjekt sein. Im Fall der Veröffentlichung ihrer persönlichen Information ohne ihre Einwilligung könnte ihr Recht auf informationelle Selbstbestimmung oder ihr Recht auf Privatsphäre das Schutzobjekt sein. Im Fall des ungeeigneten Kommentars über die Zielperson könnte ihre Ehre das Schutzobjekt sein. 



\section{$\$ 3$ Der Schutz des Rechts am eigenen Bild}

\section{Der Schutz des Rechts am eigenen Bild in Deutschland}

\section{Die Entstehung des Schutzes}

Die gesetzlichen Bestimmungen zum Schutz des Rechts am eigenen Bild in Deutschland bestehen hauptsächlich im „Gesetz betreffend das Urheberrecht an Werken der bildenden Künste und der Photographie (Kunsturheberrechtsgesetz, kurz: KunstUrhG)“ vom 9. Januar 1907. Der Auslöser des KunstUrhG war der erster „Paparazzi“-Fall, in dem die Fotografen in das Sterbezimmer Otto von Bismarcks eingedrungen und den Leichnam fotografiert hatten. ${ }^{152}$ Damals konnte das RG die Verbreitung der Fotos nur wegen Hausfriedensbruch untersagen. ${ }^{153}$ Die Regelungslücke wurde durch das KunstUrhG geschlossen, in dem der Achtungsanspruch der Persönlichkeit und das Informationsinteresse der Allgemeinheit einen Ausgleich finden. ${ }^{154}$ Dieser Ausgleich stellt sich in $\$ \$ 22$ und 23 des KunstUrhG als ein Drei-Stufen-Schutz des Rechts am eigenen Bild dar.

\section{Drei-Stufen-Schutz}

Zum Schutz des Rechts am eigenen Bild sind in den $\$ \$ 22$ und 23 des KunstUrhG drei Stufen geregelt. Auf Stufe 1 dürfen Bildnisse prinzipiell nur mit Einwilligung des Abgebildeten bzw. des Rechtsinhabers verbreitet oder öffentlich zur Schau gestellt werden ( $\$ 22$ Satz 1 KunstUrhG). Die Einwilligung kann auch in Form von Abbilden-Lassen sein ( $\$ 22$ Satz 2 KunstUrhG). Auf Stufe 2 dürfen manche Bildnisse ausnahmsweise ohne Einwilligung verbreitet und zur Schau gestellt werden ( $\$ 23$ Abs. 1, Satz 1 KunstUrhG). Dazu gehören erstens die Bildnisse aus dem Bereiche der Zeitgeschichte; zweitens die Bilder, auf denen die Personen nur als Beiwerk neben einer Landschaft oder sonstigen Örtlichkeit erscheinen; drittens die Bilder von Versammlungen, Aufzügen und ähnlichen Vorgängen, an denen die dargestellten Personen teilgenommen haben; viertens die Bildnisse, die nicht auf Bestellung angefertigt sind, sofern die Verbreitung oder Schaustellung einem höheren Interesse der Kunst dient ( $\$ 23$ Abs. 1, Nr. 1-4 KunstUrhG). Auf Stufe 3 dürfen die Verbreitung und Schaustellung der Bildnisse aufgrund

152 Wandtke/Bullinger, KunstUrhG $\$ 22$ Rn. 1.

153 RGZ 45, 170.

154 Vgl. BVerfG GRUR 2000, 446, 451 und Wandtke/Bullinger, KunstUrhG $\$ 22$ Rn. 1. 
von Stufe 2 das berechtigte Interesse des Abgebildeten und des anderen Rechtsinhabers nicht verletzen ( $\$ 23$ Abs. 2 KunstUrhG).

Für Stufe 2 besteht auch die andere Möglichkeit, die Bildnisse ohne Einwilligung zu verbreiten oder öffentlich zur Schau zu stellen, nämlich für Zwecke der Rechtspflege und der öffentlichen Sicherheit ( $\$ 24$ KunstUrhG). Aber $\$ 24$ privilegiert nur die Tätigkeit von Behörden, nicht die von Privatpersonen. ${ }^{155}$ Für Menschenfleischsuche spielt dieser Paragraph keine Rolle.

Durch die drei Stufen kann man sehen, dass die Ausnahmen, ohne Einwilligung die Bildnisse zu verwenden, auf vier Fälle beschränkt sind. Auch für die vier Ausnahmen ist eine Abwägung der gegenläufigen Interessen im Einzelfall in Stufe 3 erforderlich. ${ }^{156}$ Das Interesse des Abgebildeten und des anderen Berechtigten wurde in Deutschland durch die $\$ \$ 22 \mathrm{ff}$. KunstUrhG ausreichend geschützt.

\section{Das Recht am eigenen Bild als ein besonderes Persönlichkeitsrecht}

\$\$ 22 ff. KunstUrhG hat zwei Schutzzwecke. ${ }^{157}$ Zum einen wird das vermögensrechtliche Interesse des Abgebildeten vor der willkürlichen kommerziellen Verwendung seines Bildes geschützt. ${ }^{158} \mathrm{Zum}$ anderen schützt es das persönlichkeitsrechtliche Interesse des Abgebildeten ${ }^{159}$, nämlich das Selbstbestimmungsrecht über seine Darstellung. ${ }^{160}$ Das Selbstbestimmungsrecht bedeutet weiterhin, dass der Abgebildete ausschließlich befugt ist, darüber zu entscheiden, ob und in welcher Weise er der Öffentlichkeit im Bild vorgestellt wird. ${ }^{161}$ Dies bedeutet allerdings nicht, dass der Abgebildete den Anspruch darauf hat, nur so dargestellt zu werden, wie er sich selbst sieht oder gesehen werden will. ${ }^{162}$

Weil das Recht am eigenen Bild vor der Erfindung des allgemeinen Persönlichkeitsrechts schon im Gesetz geregelt war, ist es als besonderes Persönlichkeitsrecht bezeichnet. ${ }^{163}$

155 Dreier/Specht in Dreier/Schulze, KunstUrhG $\$ 24$, Rn. 6.

156 Kaiser in Erbs/Kohlhaas, KunstUrhG \$33, Rn. 82.

157 Engels in Ahlberg/Götting, KunstUrhG \$22 Rn. 5; Vgl. BGH ZUM 2000, 589, 590 und BGH, GRUR 2000, 709, 712.

158 BGH, GRUR 2000, 709, 712; Engels in Ahlberg/Götting, KunstUrhG $\$ 22$ Rn. 5.

159 Engels in Ahlberg/Götting, KunstUrhG \$22 Rn. 2.

160 Engels in Ahlberg/Götting, KunstUrhG $\$ 22$ Rn. 5.

161 BGH, NJW 2007, 1977, 1977; Engels in Ahlberg/Götting, KunstUrhG \$ 22 Rn. 2.

162 BVerfG, NJW 2000, 1021, 1022.

163 BGH, GRUR 1955, 197; Nink in Spindler/Schuster, BGB \$ 823, Rn. 6. 
Menschenfleischsuche bezüglich ist das Recht am eigenen Bild nur als Persönlichkeitsrecht zu betrachten. Der vermögensrechtliche Teil wird in dieser Arbeit nicht diskutiert. Wegen der engen Beziehung mit dem allgemeinen Persönlichkeitsrecht werden die weiteren rechtlichen Probleme des Rechts am eigenen Bild später im Rahmen des Schutzes des allgemeinen Persönlichkeitsrechts diskutiert.

Wie oben gesagt, wurde das KunstUrhG ursprünglich wegen des Schutzes der Prominenten ausgelöst. In der Praxis waren die Prinzipien zum Schutz des Rechts am eigenen Bild auch sehr oft in den Prominenten betroffenen Urteilen entwickelt.Dies bezüglich wurde unter dem Teil $\S 8, \mathrm{~V}, 2$, a) diskutiert.

\section{Ansprüche wegen Verletzung des Rechts am eigenen Bild}

Als strafrechtliches Ergebnis der Rechtsverletzung im Sinne von $\$ \$ 22,23$ KunstUrhG wird der Verletzer auf Antrag des Berechtigten mit Freiheitsstrafe bis zu einem Jahr oder mit Geldstrafe bestraft ( $\$ 33$ KunstUrhG). Zivilrechtlich ist das Recht am eigenen Bild gemäß $\$ 823$ Abs. 2 BGB i.V.m. $\$ \$ 22,33$ KunstUrhG geschützt. ${ }^{164}$ Übrigens ist es für das Recht am eigenen Bild als besonderes Persönlichkeitsrecht stets möglich, auf das allgemeine Persönlichkeitsrecht zurückzugreifen. ${ }^{165}$ Deswegen wird das Recht am eigenen Bild auch als sonstiges Recht i.S.v. $\$ 823$ Abs. 1 BGB geschützt. ${ }^{166}$ Ferner sind Unterlassungs- und Beseitigungsansprüche in analoger Anwendung von $\$ 1004$ BGB und ein Schmerzensgeldanspruch i.V.m. $\$ 253$ Abs. 2 BGB möglich. ${ }^{167}$

\section{Der Schutz des Rechts am eigenen Bild in China}

Die Hauptregelung über das Recht am eigenen Bild ist in China in $\$ 100$ und $\$ 120$ der „Allgemeinen Grundsätze des Zivilrechts“ (AGZR) geregelt. Ähnlich wie der allgemeine Teil des Bürgerlichen Gesetzbuchs in Deutschland ist das AGZR das wichtigste zivilrechtliche Gesetz in China. Gemäß $\$ 100$ der AGZR hat der Bürger das Recht an seinem eigenen Bild; ohne seine Einwilligung darf das Bild eines Bürgers nicht zu einem gewerblichen Ziel verwendet werden. Wenn das Recht am eigenen Bild des Bürgers verletzt ist, kann er gemäß $\$ 120$

164 Dreier/Specht in Dreier/Schulze, KunstUrhG $\$ 50$, Rn. 6; Fricke in Wandtke/ Bullinger, KunstUrhG $\$ 22$, Rn. 28.

165 Nink in Spindler/Schuster, BGB $\$ 823$, Rn. 6.

166 Dreier/Specht in Dreier/Schulze, KunstUrhG $\$ 50$, Rn. 6; Fricke in Wandtke/ Bullinger, KunstUrhG $\$ 22$, Rn. 28.

167 Dreier/Specht in Dreier/Schulze, KunstUrhG $\$ 50$, Rn. 2. 
AGZR Ansprüche auf Unterlassung, Wiedergutmachung der Ehre, Beseitigung der Wirkung, offizielle Entschuldigung und Schadensersatz verlangen.

\section{Die Diskussion über das gewerbliche Ziel im $\$ \mathbf{1 0 0}$ der AGZR}

Wegen der Beschränkung durch gewerblichen Verwendungszweck im $\$ 100$ der AGZR wurde der Schutz des Rechts am eigenen Bild in der Praxis verringert. Das Recht würde vor der willkürlichen Verwendung von normalen Bürgern ohne gewerblichen Zweck nicht geschützt. Dies ist für Menschenfleischsuche besonders der Fall, weil bei der Verwendung des Bilds der Zielperson von dem Internetnutzer meistens kein gewerblicher Zweck besteht. Wörtlich ausgelegt wäre das Recht am eigenen Bild der Zielperson während Menschenfleischsuche nicht vom chinesischen Zivilrecht geschützt. ${ }^{168}$

Jedoch hat diese Lücke seit langem schon die Aufmerksamkeit der Juristen gewonnen. Im Jahre 1988 gab es schon die Meinung, dass die Benutzung des Bildes eines anderen ohne seine Einwilligung das Recht am eigenen Bild verletzen kann, auch wenn kein gewerblicher Zweck besteht. Nach dieser Meinung sollte der gewerbliche Zweck keine Voraussetzung der Rechtsverletzung am eigenen Bild sein. ${ }^{169}$

Aber die in dem gleichen Jahr hatten die „Ansichten des Obersten Volksgerichts zu Problemen bei der Durchsetzung und Anwendung der AGZR der V. R. China" (OVG-AGZR-Ansichten) im $\$ 139$ noch mal den gewerblichen Zweck betont. ${ }^{170}$ Diese Ansichten wurden zwar nur versuchsweise durchgeführt, spielten aber eine Rolle wie die offizielle Rechtsprechung über AGZR. In der Praxis hatten diese Ansichten eine Rechtswirkung wie ein Gesetz.

In der Justizpraxis in den folgenden Jahren hatten einige regionalen Gerichte versucht, $\$ 100$ der AGZR anders als der Gesetztext auszulegen. Ihr begegneten aber starke Hinderungen. Die meisten Gerichte hatten in der Praxis den gewerblichen Zweck als eine Voraussetzung der Rechtsverletzung am eigenen Bild akzeptiert. ${ }^{171}$

Die Diskussion in der Literatur hatte aber nicht aufgehört. Zum Beispiel hatte ein Jurist den Vorschlag gemacht, durch systematische Auslegung das Anwendungsproblem vom $₫ 100$ der AGZR auf der Verwendung des Bildes

168 Zhang Jianwen, Journal of Henan University of Economics and Law 2012, No. 2, 95, 98.

169 Lu Yixiao, Journal of Ningbo Radio \& TV Universtity 2009, No. 4, 4, 5.

170 Hou Li/Wang Xiaomi, Legal Science Monthly 1992, No. 2, 15.

171 Yang Lixin, Ren shen quan fa lun, S. 523. 
ohne gewerblichen Zweck zu lösen. Nach seiner Meinung steht $₫ 100$ AGZR unter dem Kapitel 5 Abschnitt 4, in dem die Regelungen über Persönlichkeitsrechte vom zivilrechtlichen Aspekt stehen. Dieser Abschnitt zielt darauf, durch die Beschreibung der unterschiedlichen Persönlichkeitsrechte diese zu unterscheiden und zu sortieren. Durch die Regelungen in diesem Abschnitt sind die Rechte der Bürger positiv verteilt. Die Regelungen über Rechtsverletzungen und Haftungen stehen in Kapitel 6. Deswegen ist ein gewerblicher Zweck keine strenge Voraussetzung für jede Rechtsverletzung am eigenen Bild, ${ }^{172}$ sondern nur ein Beispiel von den Fällen der Rechtsverletzung. Diese Meinung wurde langsam von der Justizpraxis akzeptiert und ist die herrschende Meinung geworden. ${ }^{173}$

Zwischenzeitlich herrscht in der Literatur auch die Meinung, dass das Recht am eigenen Bild in zwei Teile einsortiert werden soll: der Teil vom Persönlichkeitsrecht und der Teil vom Vermögensrecht. ${ }^{174}$ Nach dieser Meinung kann das Anwendungsproblem des $₫ 100$ der AGZR auch erfolgreich gelöst werden. Die gewerbliche Verwendung des Bildes eines Anderen ohne seine Einwilligung hat offensichtlich den Teil vom Vermögensrecht verletzt. Wenn die Verwendung des Bildes ohne gewerblichen Zweck zu einem Schaden führt, soll es als eine Verletzung von dem Teil des Persönlichkeitsrechts behandelt werden. Bei Menschenfleischsuche ist der Schutz des Rechts am eigenen Bild auch vorrangig vom Aspekt des Persönlichkeitsrechts diskutiert.

Zusammengefasst wird der gewerbliche Zweck als eine allgemeine Voraussetzung der Rechtsverletzung des Rechts am eigenen Bild von der Literatur und von der Justizpraxis verneint.

\section{Ein-Stufen-Schutz}

Aus $₫ 100$ der AGZR kann man entnehmen, dass das chinesische Recht nur eine Stufe zum Schutz des Rechts am eigenen Bild gesetzlich geregelt hat. Die Fälle von angemessener Verwendung des Bildes ohne die Einwilligung des Rechtsinhabers sind nur in der Literatur diskutiert und wurden durch Entscheidungspraxis entwickelt.

$\mathrm{Zu}$ berücksichtigen ist ein „Handbuch über die Eröffnung des Gerichtsverfahrens", das als interne Richtlinie für die Arbeit der chinesischen Richter bereitgestellt ist. Das Handbuch hat 30 Kriterien zur Eröffnung eines

172 Vgl. Peng Juan, Journal of Political Science and Law 2004, No. 3, $23 \mathrm{f}$.

173 Vgl. Liu, Haitao/Zheng, Jinxiong/Shen, Rong, S. 673.

174 Liu Deliang, S. 309. 
Gerichtsverfahrens gelistet. $\$ 24$ davon sind Kriterien über die Verwendung der Bildnisse ohne die Einwilligung des Abgebildeten. Die Verwendung des Bildes eines Bürgers ohne seine Einwilligung begründet gemäß $\$ 24$ keine Verletzung des Rechts am eigenen Bild, wenn die Verwendung für Zwecke des öffentlichen Interesses, des eigenen Interesses des Bürgers, der Rechtspflege, der Berichterstattung, der wissenschaftliche Forschung oder der Bildung ist. Jedoch bietet das Handbuch nur einen Vorschlag, der keine rechtliche Wirkung hat. In der Entscheidungspraxis entscheiden die Richter nach ihren Kenntnissen und Erfahrungen, ob eine angemessene Verwendung der Bildnisse begründet ist.

Wenn die zweite Stufe schon im Gesetz fehlt, sollte man selbstverständlich nicht erwarten, dass die dritte Stufe besteht. Zum Schutz des Rechts am eigenen Bild in China bedarf es dringend gesetzliche Regelungen.

\section{Schutz des Rechts am eigenen Bild durch den Schutz der Privatsphäre}

Zu erwähnen ist, dass die Rechte der Zielperson um ihre Bilder nicht nur durch den Schutz des Rechts am eigenen Bild verwirklicht werden können, sondern es besteht auch eine andere Schutzmöglichkeit. Die Verwendung des Bildes des Rechtsinhabers ohne seine Einwilligung kann auch als eine Verletzung des Rechts auf Privatsphäre behandelt werden, ${ }^{175}$ besonders wenn sein Recht auf Gelassenheit des Lebens durch die Verwendung des Bildes verletzt ist.

175 Zhang Jianwen, Journal of Henan University of Economics and Law 2012, No. 2, 95, 98. 


\section{$\$ 4$ Der Schutz des Rechts auf informationelle Selbstbestimmung}

\section{Der Schutz des Rechts auf informationelle Selbstbestimmung in Deutschland}

Während der Menschenfleischsuche werden die persönlichen Informationen der Zielperson von den Internetnutzern oder dem ICP auf die Webseite des ISP oder des ICP gestellt. Gehörten diese Informationen zu den personenbezogenen Daten, würden sie durch das Recht auf informationelle Selbstbestimmung geschützt werden.

\section{Das Recht auf informationelle Selbstbestimmung und BDSG}

Das Recht auf informationelle Selbstbestimmung, das aus dem allgemeinen Persönlichkeitsrecht ${ }^{176}$ stammt, ist ein erfundener Begriff des Bundesverfassungsgerichts in dem so genannten Volkszählungsurteil ${ }^{177}$ aus dem Jahr 1983.

Das Recht findet seine Grundlage in Art. 2 Abs. 1 i.V.m. Art. 1 Abs. 1 des GG. Geschützt wird das Recht „grundsätzlich selbst über die Preisgabe und Verwendung seiner persönlichen Daten zu bestimmen und zu wissen, wer was, wann und bei welcher Gelegenheit über ihn weiß“. ${ }^{178}$ „Das Recht auf informationelle Selbstbestimmung macht den Einzelnen grundsätzlich zum Herrn der ihn betreffenden Daten “179. Es ermöglicht dem Einzelnen weitgehende Rechte, „über die Verwendung seine personenbezogenen Daten zu entscheiden“180.

Das Recht auf informationelle Selbstbestimmung sollte ursprünglich vor der willkürlichen Benutzung der persönlichen Informationen durch die Regierung schützen. Nach und nach wird die gewerbliche Stelle, die aufgrund eigenen wirtschaftlichen Interesses die persönlichen Informationen missbraucht, auch Adressat des Rechts. ${ }^{181}$ Als das Hauptgesetz zum Schutz des Rechts auf informationelle Selbstbestimmung richtet BDSG hauptsächlich auf die zwei Adressaten.

176 Art. 2 Abs. 1 GG i.V.m. Art. 1 Abs. 1 GG.

177 BVerfGE 65, 1 = NJW 1984, 419.

178 BVerfGE 65, 1, 43 = NJW 1984, 419, 422; Vgl. Seidel/Nink, CR 2009, 666, 669.

179 Gola/Schomerus in Gola/Schomerus, BDSG $\$ 1, \mathrm{Rn} .10$.

180 Gola/Schomerus in Gola/Schomerus, BDSG § 1, Rn. 10.

181 Vgl. Gola/Schomerus in Gola/Schomerus, BDSG \$1, Rn. $12 f$. 


\section{Die Anwendbarkeit des BDSG auf die Fälle in dieser Arbeit}

Breits im Jahre 2003 hat der EuGH schon bejaht, dass das Datenschutzrecht auf die Veröffentlichungen im Internet anwendbar ist. ${ }^{182}$ Im Folgenden wird die Anwendbarkeit des BDSG auf das Verhalten der Internetnutzer, des ICP und des ISP während Menschenfleischsuche geprüft. Aber zuerst wird erklärt, welche persönliche Informationen bzw. personenbezogene Daten zu dem Schutzgegenstand des BDSG gehören.

\section{a) Die Eingrenzung personenbezogener Daten}

Personenbezogene Daten sind gemäß $₫ 3$ Abs. 1 BDSG Einzelangaben über persönliche oder sachliche Verhältnisse einer bestimmten oder bestimmbaren natürlichen Person ${ }^{183}$. In der Praxis ist der Begriff von personenbezogenen Daten sehr weit ausgelegt. Dazu gehören nicht nur der Name, die Kontaktmöglichkeit, die Anschrift, die Tätigkeit des Betroffenen ${ }^{184}$ sondern auch die Bewertung über den Betroffenen und seine Tätigkeit ${ }^{185}$.

Aber die Bewertung bzw. das Werturteil über eine natürliche Person muss sich mindestens auf Tatsachenbehauptungen beziehen oder nach der herrschenden Meinung der Darstellung persönlicher und sachlicher Verhältnisse einer Person dienen ${ }^{186}$. Wenn sich die Meinungsäußerungen ausschließlich um Werturteile und nicht um Tatsachenbehauptungen handelt, enthalten sie keine personenbezogenen Daten i.S.v. $\$ 3$ Abs. 1 BDSG, ${ }^{187}$ weil es in diesem Fall an Informationswert über eine Person vollständig mangelt. ${ }^{188}$

Die Unterscheidung zwischen der Tatsachenbehauptung und des Werturteiles in einer Äußerung ist für die weitere Prüfung entscheidend. Im Fall der Einbeziehung von Werturteilen über eine Person in den Anwendungsbereich des Datenschutzrechts besteht die Gefahr der Einschränkung der Meinungsfreiheit i.S.v. Art. 5 Abs. 1 GG. ${ }^{189}$

182 EuGH, EuR 2004, 291, $297 f$.

183 Ballhausen/Roggenkamp, K\&R 2008, 403, 406.

184 Legaler Begriff siehe: $₫ 3$ Abs. 1 BDSG.

185 BGH, BGHZ 181, 328, Rn. 17 = NJW 2009, 2888, $2890=$ MMR 2009, 608, 609.

186 Härting, CR 2009, 21, 26; Gola/Schomerus, BDSG, \$ 3 Rn. 6 f.

187 Härting, CR 2009, 21, 28.

188 Härting, CR 2009, 21, 26; Gola/Schomerus, BDSG, § 3 Rn. 6.

189 Härting, CR 2009, 21, 26. 
b) Die Anwendbarkeit des BDSG auf die von den Internetnutzern begangenen Rechtsverletzungen

Gemäß $₫ 1$ Abs. 2 Nr. 3 gilt BDSG „für die Erhebung, Verarbeitung und Nutzung personenbezogener Daten durch nicht-öffentlichen Stellen,... es sei denn, die Erhebung, Verarbeitung oder Nutzung der Daten erfolgt ausschließlich für persönliche oder familiäre Tätigkeiten“. Nicht-öffentliche Stellen sind gemäß $§ 2$ Abs. 4 BDSG natürliche und juristische Personen. Die Internetnutzer als natürliche Personen zählen als nicht-öffentliche Stellen i.S.v. BDSG. Zur Anwendbarkeit des BDSG im Fall der beliebigen Veröffentlichung personenbezogener Daten durch den Internetnutzer kommt es zum Schluss auf die Frage an: Gehört das Verhalten der Internetnutzer während Menschenfleischsuche zu den „persönlichen oder familiären Tätigkeiten"?

Persönliche oder familiäre Tätigkeiten als eine Ausnahme von dem Anwendungsbereich des BDSG hat der Gesetzgeber in Deutschland aus der EURichtlinie übernommen. ${ }^{190}$ Der Hintergedanke des Gesetzgebers liegt darin, dass derjenigen, der wegen persönlicher oder familiärer Tätigkeiten personenbezogene Daten erhebt oder verwendet, ebenso schutzbedürftig wie die Betroffenen ist ${ }^{191}$. Im Gegensatz dazu sind die Betroffenen im Vergleich mit den zwei Adressaten des BDSG, nämlich der öffentlichen Stelle und der nicht-öffentlichen Stelle (persönliche oder familiäre Tätigkeiten ausgenommen), schutzwürdiger.

Dies kann man aus der Entwicklung der Gesetztexte deutlicher ersehen. Vor 2001 war diese Ausnahme als „geschäftsmäßig oder für berufliche oder gewerbliche Zwecke" positiv formuliert. ${ }^{192}$ Das heißt, vom BDSG geregelt ist nur die Datenbearbeitung der nicht-öffentlichen Stelle für geschäftsmäßige, berufliche oder gewerbliche Zwecke. Die nicht-öffentliche Stelle in diesem Sinne hat mit der öffentliche Stelle eine offensichtliche Gemeinsamkeit: ihr Verhältnis mit dem Betroffenen ist hierarchisch.

Die öffentliche Stelle wie z.B. die Regierung ist offensichtlich mächtiger als der normale Bürger. Der Betroffene ist die schwächere Seite. Die gleiche Situation besteht in dem Verhältnis zwischen dem Betroffenen und der nicht-öffentlichen Stelle, wenn sie mit dem Betroffenen auf Grund eines geschäftlichen oder beruflichen oder ähnlichen Verhältnisses verbunden ist. Die Verhältnisse zeigen sich als Unternehmer-Verbraucher-Verhältnis, Arbeitgeber-ArbeitnehmerVerhältnis oder anderes Verhältnis mit ähnlicher Situation. Entweder als

190 Vgl. Dammann/Simitis, EG-Datenschutzrichtlinie, Art. 3 Rn. 7 f.

191 Simitis in Simitis, BDSG § 1, Rn. 149.

192 Simitis in Simitis, BDSG $₫ 1$, Rn. 147. 
Verbraucher oder als Arbeitnehmer steht der Betroffene in dem Verhältnis auf der schwächeren Position.

Im Gegensatz dazu besteht innerhalb eines persönlichen oder familiären Verhältnisses keine Hierarchie. Die Teilnehmer an den persönlichen oder familiären Tätigkeiten verdienen datenrechtlich gleiches Schutzniveau.

Mit der neuen negativen Formulierung im $₫ 1$ Abs. 2 Nr. 3 ist der Anwendungsbereich des BDSG klarer geworden. ${ }^{193}$ Dies berührt aber nicht den Grundgedanken über das Ziel dieser Vorschrift, den Bereich persönlicher Lebensführung von der beruflichen und geschäftlichen Sphäre abzugrenzen ${ }^{194}$, was in der alten Fassung deutlicher dargestellt wird. Der Datenumgang wegen persönlicher oder familiärer Tätigkeiten wird nicht vom BDSG, sondern von den allgemeinen Vorschriften geregelt. ${ }^{195}$

Die Beurteilung der persönlichen oder familiären Tätigkeiten richtet sich nach der Verkehrsanschauung. ${ }^{196}$ Dafür ist eine persönliche oder familiäre Beziehung zwischen dem Täter und den Betroffenen nicht notwendig. ${ }^{197}$ Die Internetnutzer während Menschenfleischsuche haben kein gewerbliches, geschäftliches oder berufliches Interesse an den persönlichen Daten der Zielperson. Die Erhebung oder Verwendung der Daten ist, wie vorher gesagt wurde, aus Ärger oder wegen Neugier oder zum Spaß. Dies gehört eher zum Bereich der persönlichen Lebensführung. BDSG findet auf das Verhalten der Internetnutzer während Menschenfleischsuche keine Anwendung.

\section{c) Die Anwendbarkeit des BDSG auf der von den ICP begangenen Rechtsverletzung}

Während der Menschenfleischsuche werden die persönliche Informationen der Zielperson auf der Webseite des ICP erscheinen. Die Offenlegung dieser Informationen kann entweder von dem ICP selbst oder mit seiner nachherigen Überprüfung von seinen Internetnutzern durchgeführt werden.

Anders als bei Internetnutzern ist BDSG auf das Verhalten des ICP anwendbar. Gemäß $\$ 1$ Abs. 2 Nr. 3 gilt BDSG für „die Erhebung, Verarbeitung und Nutzung personenbezogener Daten durch nicht-öffentlichen Stellen, soweit sie die Daten unter Einsatz von Datenverarbeitungsanlagen verarbeiten, nutzen oder

193 Gola/Schomerus in Gola/Schomerus, BDSG $₫ 1$, Rn. 21.

194 Simitis in Simitis, BDSG $\$ 1$, Rn. 149.

195 Simitis in Simitis, BDSG \$ 1, Rn. 149.

196 Simitis in Simitis, BDSG $₫ 1$, Rn. 151.

197 Simitis in Simitis, BDSG § 1. Rn. 152. 
dafür erheben oder die Daten in oder aus nicht automatisierten Dateien verarbeiten, nutzen oder dafür erheben“.

\section{aa) Erhebung, Verarbeitung und Nutzung personenbezogener Daten}

Solange die personenbezogenen Daten auf der Webseite des ICP erscheinen, ist die Erhebung oder Verarbeitung oder mindestens Nutzung personenbezogener Daten von dem ICP begründet. Es ist nicht notwendig, um den wirkliche Willen des ICP zu forschen, ob er die Absicht hat, bezügliche personenbezogene Daten zu erheben, zu verarbeiten oder zu nutzen. Hier soll die Erklärungstheorie angewandt werden, weil der ICP Experte für den Datenumgang ist, während es dem normalen Bürger an Fachkenntnissen mangelt, was beim Datenumgang auf der Seite des ICP mit dem Daten passiert.

\section{bb) Nicht-öffentliche Stellen}

Nach $\$ 2$ Abs. 4 BDSG sind nicht-öffentliche Stellen natürliche und juristische Personen, Gesellschaften und andere Personenvereinigungen des privaten Rechts. Egal ob die Webseite von einer natürlichen Person oder von einer Gesellschaft betrieben ist, gehört der Betreiber zweifellos zu den nicht-öffentlichen Stellen.

\section{cc) Einsatz von Datenverarbeitungsanlagen}

Eine Datenverarbeitungsanlage ist eine Anlage zum automatischen Datenumgang. ${ }^{198}$ Der Computer ist z.B. eine typische Form einer Datenbearbeitungsanlage. Es ist schon deutlich, dass der Betrieb einer Webseite durch Computer ermöglicht ist. Die Leistungsfähigkeit der Computer spielt für die Identifizierung der Datenverarbeitungsanlage keine Rolle, solange er zur Datenverarbeitung aufgebaut ist. ${ }^{199}$ Übrigens ist der Begriff Datenverarbeitungsanlage wegen der vielfaltigen Geräte heutzutage außerordentlich weit ausgelegt. ${ }^{200}$ Zum Betreiben einer Webseite ist eine Datenverarbeitungsanlage zwingend nötig.

$\mathrm{Zu}$ diskutieren ist, ob die manuelle Erhebung personenbezogenen Daten vor dem Einsatz der Datenverarbeitungsanlage auch zu dem Anwendungsbereich des BDSG gehört. Dies ist für Menschenfleischsuche besonders der Fall, weil die Offenlegung der Information der Zielperson häufig in der Form von Nachrichten, Blog-Texten oder Beiträgen der Internetnutzer im Internet verwirklicht

198 Simitis in Simitis, BDSG $\$ 1$, Rn. 140.

199 Ernestus in Simitis, BDSG $\$ 9$, Rn. 72.

200 Simitis in Simitis, BDSG $\$ 1$, Rn. 140. 
ist, welche häufig manuell verfasst sind. Nach der herrschenden Meinung gehört manuelle Erhebung personenbezogenen Daten auch zu dem Anwendungsbereich des BDSG, wenn ein Zweck um den Zeitpunkt der Erhebung besteht, eine spätere automatische Behandlung durchzuführen. ${ }^{201}$ Nach dem manuellen Verfassen des Artikels, der die personenbezogenen Daten enthält, wird er durch Datenverarbeitungsanlagen gespeichert, publiziert oder durch andere Weise verarbeitet. Der Einsatz von Datenverarbeitungsanlagen ist auch in diesem Fall begründet.

\section{dd) Kein persönlicher oder familiärer Zweck}

Wie oben gesagt, spielt ein geschäftlicher, gewerblicher oder beruflicher Zweck der Datenerhebung oder Datenverarbeitung seit 2001 für die Anwendbarkeit des BDSG keine Rolle mehr. Egal ob ein ICP durch den Betrieb der Webseite Geld verdienen möchte, solange er dadurch keinen persönlichen oder familiäreren Zweck nachgeht, muss BDSG auf seinen Betrieb angewandt werden.

Wie oben schon erwähnt, liegt der Hintergedanke über persönliche oder familiäre Tätigkeiten darin, dass derjenigen, der wegen persönlicher oder familiärer Tätigkeiten personenbezogene Daten erhebt oder verwendet, ebenso schutzbedürftig wie die Betroffenen ist ${ }^{202}$. Durch die Regelung möchte der Gesetzgeber den Bereich persönlicher Lebensführung von der beruflichen und geschäftlichen Sphäre abgrenzen ${ }^{203}$. Es ist selten, dass ein ICP nur für persönlichen oder familiären Zweck eine Webseite betreibt. Jedoch müssen die Sonderfälle berücksichtigt werden.

\section{ee) Zwischenergebnis}

Nach der Analyse ist festzustellen, dass das BDSG auf das Verhalten des ICP während Menschenfleischsuche anwendbar ist, solange das Verhalten nicht zu den persönlichen oder familiären Tätigkeiten gehört.

Die Vorschriften, die auf die Situation von ICP anzuwenden sind, befinden sich in den $\$ \$ 27$ ff. BDSG. Davon sind zwei Vorschriften für die Zulässigkeit des Datenumgangs von ICP entscheidend. Eine ist $\$ 28$ BDSG, nämlich Datenerhebung und -speicherung für eigene Geschäftszwecke. Die andere ist $\$ 29$ BDSG, nämlich geschäftsmäßige Datenerhebung und -speicherung zum Zweck der

201 Simitis in Simitis, BDSG $\$ 1$, Rn. 141.

202 Simitis in Simitis, BDSG $₫ 1$, Rn. 149.

203 Simitis in Simitis, BDSG $₫ 1$, Rn. 149. 
Übermittlung. Welche der beiden Vorschriften auf die Situation von ICP während Menschenfleischsuche angewendet werden kann, soll jetzt geprüft werden.

\section{ff) Die Anwendbarkeit des $\$ 28$ BDSG auf der vom ICP begangenen Rechtsverletzung während Menschenfleischsuche}

$\$ 28$ BDSG regelt die Fälle der Verwendung personenbezogener Daten für eigene Geschäftszwecke. ${ }^{204}$ Zur Erfüllung eigener Geschäftszwecke ist es erforderlich, dass die Datenverarbeitungen als Hilfsmittel zur Erfüllung bestimmter anderer, eigener Zwecke der datenverarbeitenden Stelle erfolgt, ohne selbst das geschäftliche Interesse zu bilden. ${ }^{205}$

Wie oben gesagt, sind die auf der Webseite von ICP erscheinenden personenbezogenen Daten, wie die anderen Inhalte auf der Webseite, für die Benutzung der Internetnutzer bereitgestellt. Der ICP möchte nicht die Daten nutzen, um sich mit dem Betroffenen in Verbindung zu setzen oder um sie als Kunden zu gewinnen ${ }^{206}$. Die Veröffentlichung der Daten zielt nur darauf, sie an den Internetnutzer weiterzuleiten und zu verbreiten ${ }^{207}$. Das abgezielte Verhältnis besteht nur zwischen dem ICP und den Nutzern seines Dienstes. Bevor eine Rechtsverletzung vorgenommen ist, haben der ICP und der Betroffene der personenbezogenen Daten keine gesetzliche Verbindung.

Deswegen ist die Datenerhebung und -speicherung in unserem Fall nur zum Ziel der Übermittelung an die Internetnutzer. Ein weiterer Zweck existiert nicht. Die Daten haben sich in Ware verwandelt und sind selbst Geschäftsgegenstand geworden. ${ }^{208}$ Genau diese Daten bilden das geschäftliche Interesse des ICP.

Es könnte sein, dass der ICP durch Datenverarbeitung bzw. Datenübermittelung Geld verdienen (Mehr Informationen auf der Webseite einzutragen um mehr Leser zu gewinnen, und darum einen besserer Preis von Werbungen zu bekommen) möchte, welcher ein allgemeines Ziel des Betriebs einer Webseite und gleichzeitig ein anderer Geschäftszweck i.S.v. \$28 BDSG wäre. Aber der Geschäftszweck im Sinne des BDSG bezieht sich nur auf den Betriebs-Modus der Webseite. Geldverdienen ist kein Geschäftszweck im Sinne des BDSG. ${ }^{209}$ Zudem

204 OLG Frankfurt, CR 2012, 399; Simitis in Simitis, BDSG § 28, Rn. 22.

205 Gola/Schomerus in Gola/Schomerus, BDSG $₫ 28$, Rn. 4; siehe auch Wedde in: Däubler, $₫ 28$ BDSG, Rn. 10; Ballhausen/Roggenkamp, K\&R 2008, 403, 407.

206 Ballhausen/Roggenkamp, K\&R 2008, 403, 407; OLG Frankfurt, CR 2012, 399.

207 OLG Frankfurt, CR 2012, 399.

208 OLG Frankfurt, CR 2012, 399; siehe auch Simitis in Simitis, BDSG § 28, Rn. 22.

209 BGH, BGHZ 181, 328, Rn. 24 = NJW 2009, 2888, 2891 = MMR 2009, 608, 610. 
hat der BGH in seiner „spickmich-Entscheidung “210 festgestellt, dass - trotz Verbreitung von Werbeanzeigen - der Anwendungsbereich des $₫ 28$ BDSG nicht eröffnet ist. ${ }^{211}$

Die von dem ICP begangene Rechtsverletzung während Menschenfleischsuche wird nicht von $\$ 28$ BDSG reguliert.

gg) Die Anwendbarkeit des $\$ 29$ BDSG auf die vom ICP begangene Rechtsverletzung während Menschenfleischsuche

$\$ 29$ BDSG regelt den Fall der Datenverarbeitung zum Zweck der Übermittlung. Das Übermitteln personenbezogener Daten ist gemäß $₫ 3$ Abs. 4 Nr. 3b BDSG das Bekanntgeben gespeicherter oder durch Datenverarbeitung gewonnener personenbezogener Daten an einen Dritten u.a. in der Weise, dass der Dritte zur Einsicht oder zum Abruf bereitgehaltene Daten einsieht oder abruft. ${ }^{212}$

Wie in den letzten Abschnitten erklärt, zielt die Veröffentlichung der personenbezogenen Daten der Betroffenen auf der Webseite des ICP nicht darauf, um mit den Betroffenen in Kontakt zu stehen oder zu treten. Der ICP erhebt und speichert die Daten, damit die Nutzer die Daten einsehen oder abrufen können. Das ist die Datenübermittelung i.S.v. $\$ 29$ BDSG. ${ }^{213}$

Hier ist ein Vergleich mit der "Spick-mich“-Entscheidung des BGH angebracht. Obwohl dieses "Spick-mich“-Lehrerbewertungsportal eine andere Betriebsweise als der ICP hat, haben sie Gemeinsamkeiten bei der Datenübermittelung. Sie haben beide die personenbezogenen Daten erhoben und gespeichert, weil sie diese „der interessierten Allgemeinheit zur Information und zum Meinungsaustausch zur Verfügung stellen wollen. ${ }^{214}$ Ohne Übermittlungen der Informationen kann der Geschäftszweck solcher Webseite überhaupt nicht erreicht werden. ${ }^{215}$ Der BGH ist in der Entscheidung über die Zulässigkeit des „Spick-mich“-Lehrerbewertungsportals deutlich davon ausgegangen, dass der Anwendungsbereich des $₫ 29$ BDSG eröffnet ist. ${ }^{216}$

$\$ 29$ BDSG ist auf der vom ICP begangenen Rechtsverletzung während Menschenfleischsuche anwendbar.

210 BGH, BGHZ 181, 328 = NJW 2009, $2888=$ MMR 2009, 608.

211 OLG Frankfurt, CR 2012, 399, 400.

212 OLG Hamburg, CR 2012, 188, 189.

213 OLG Frankfurt, CR 2012, 399; siehe auch Simitis in Simitis, BDSG § 28, Rn. 22.

214 OLG Frankfurt, CR 2012, 399.

215 Ehmann in Simitis, BDSG $\$ 29$, Rn. 93.

216 BGH, BGHZ 181, 328, Rn. 23ff. = NJW 2009, 2888, 2891 = MMR 2009, 608, 610. 


\section{d) Die Anwendbarkeit des BDSG auf das Verhalten des ISP}

während Menschenfleischsuche

Während Menschenfleischsuche werden die personenbezogenen Daten der Zielperson auf der Webseite vom ISP auftauchen. Sie sind nicht vom ISP selbst, sondern von seinen Internetnutzern erhoben. Aber auf Grund von $₫ 3$ Abs. 7 BDSG soll eine verantwortliche Stelle diejenige sein, die die personenbezogenen Daten für sich selbst erhebt. Der Internetnutzer hat offensichtlich die von ihm offengelegten Daten für sich selbst erhoben, während sein Verhalten jedoch nicht zum Anwendungsbereich des BDSG gehört. Die Anwendbarkeit des BDSG auf das Verhalten des ISP kommt auf die Frage an, ob ISP eine rechtmäßige verantwortliche Stelle ist, die die personenbezogenen Daten für sich selbst erhebt.

Das OLG Hamburg hat in seiner Entscheidung von 2011 festgestellt, dass der ISP die rechtmäßige Verantwortliche Stelle ist, wenn die streitigen Daten von einem außenstehenden Dritten in eine vom ISP betriebene Webseite eingestellt worden sind. ${ }^{217}$ Das Gericht meinte, das Betreiben der Webseite erfolgte in erster Linie im eigenen unternehmerischen Interesse. ${ }^{218}$ Die Datenbearbeitung auf der Webseite ist für das unternehmerische Interesse des ISP, auch wenn sie von dem Internetnutzer begangen ist. „Für eigenes unternehmerisches Interesse “ wird als „für sich selbst“ i.S.v. $\$ 3$ Abs. 7 BDSG angesehen. ${ }^{219}$

Ferner hat das Gericht festgestellt, dass der Begriff von verantwortlicher Stelle nach der Konzeption des Datenschutzrechts weit zu fassen ist. ${ }^{220}$ "Jedes Unternehmen für Verarbeitungstätigkeiten, die in seinem Tätigkeits- und Haftungsbereich stattfinden, soll rechtlich verantwortlich sein und diese Verantwortung erst dann verlieren, wenn es in tatsächlicher Hinsicht keine Möglichkeit mehr hat, auf den Verarbeitungsvorgang einzuwirken, etwa weil ein isolierter physischer Datenträger sich nicht mehr in dem Besitz von Personen befindet, auf die das Unternehmen Einfluss nehmen könnte". 221 Die Datenverarbeitung der Internetnutzer liegt offensichtlich innerhalb dem Tätigkeitsbereich des ISP, der die Fähigkeit hat, auf den Verarbeitungsvorgang jeder Zeit einzuwirken. Der ISP ist die qualifizierte verantwortliche Stelle i.S.v. $\$ 3$ Abs. 7 BDSG, und soll für die DatenVerarbeitungstätigkeiten auf seiner Webseite verantwortlich sein.

217 OLG Hamburg, CR 2012, 188, 190.

218 Vgl. OLG Hamburg, CR 2012, 188, 190.

219 Vgl. OLG Hamburg, CR 2012, 188, 190.

220 OLG Hamburg, CR 2012, 188, 189.

221 OLG Hamburg, CR 2012, 188, 189; Vgl. Dammann in Simitis, BDSG § 3, Rn. 225. 
Analogisiert von der Diskussion über ICP kann man feststellen, dass $\$ 29$ BDSG auf die Situation des ISP während Menschenfleischsuche anwendbar ist, weil die Datenerhebung und -bearbeitung von ihm durchaus zum Zweck der Übermittlung an seine Internetnutzer sind.

\section{Die Anwendbarkeit der anderen Gesetze}

Das BDSG ist gemäß $\$ 1$ Abs. 3 BDSG sogenanntes „Auffanggesetz“ ${ }^{222}$ Das heißt, dass das BDSG erst anzuwenden ist, wenn keine spezifischen Vorschriften existieren, die auf den Sachverhalt anwendbar sind, der auch Gegenstand der Regelung des BDSG ist. ${ }^{223}$ Die möglichen spezifischen Vorschriften bestehen im Telekommunikationsgesetz (TKG), Telemediengesetz (TMG), Staatsvertrag für Rundfunk und Telemedien (RStV).

$\mathrm{Zu}$ erwähnen ist auch, dass das BDSG keine abschließende und ausschließliche Regelung ist. ${ }^{224}$ Der Zugriff zu den allgemeinen Vorschriften ist unter bestimmten Voraussetzungen möglich. Dies wäre besonders der Fall, wenn es um immateriellen Schadensersatz geht.

Das deutsche Recht erkennt bislang kein absolutes „Recht am eigenen Datum“ als sonstiges Recht im Sinne des $\$ 823$ Abs. 1 BGB an. ${ }^{225}$ Das Recht auf informationelle Selbstbestimmung wird aber als eine Ausprägung des allgemeinen Persönlichkeitsrechts angesehen, welches nach der herrschenden Meinung als „sonstiges Recht“ den Schutz des $₫ 823$ Abs. 1 BGB genießt. ${ }^{226}$ Aber $₫ 823$ Abs. 1 BGB kann nicht sofort auf alle deliktische Fälle angewendet werden, weil das BDSG eigentlich eine eigene Anspruchsgrundlage für Schadensersatz in $\$ 7$ hat. Nach der Rechtsprechung des BGH scheidet ein deliktischer Schadensersatzanspruch aus $\$ 823$ I BGB aus, soweit das BDSG die Rechte des Betroffenen aus unzulässiger Datenverarbeitung abschließend regelt. ${ }^{227}$ Im Gegenteil bleibt immer dann Raum für Schadensersatzansprüche aus $₫ 823$ Abs. 1 BGB wegen Verletzung des allgemeinen Persönlichkeitsrechts, wenn das BDSG eine

222 Gola/Schomerus in Gola/Schomerus, BDSG $₫ 1$, Rn. $23 \mathrm{ff}$.

223 Gola/Schomerus in Gola/Schomerus, BDSG $\$ 1$, Rn. 24.

224 Gola/Schomerus in Gola/Schomerus, BDSG $\$ 7, \mathrm{Rn} .16$.

225 Hager in Staudinger, BGB, 1999, § 823 Rn. C 173; Mertens in MünchKomm, BGB, Bd. 5, 1997, § 823 Rn. 155; Neunhoeffer, 201.

226 BGH, BGHZ 91, 233, 238f.; Sprau in Palandt, BGB, 2015, §823 Rn. 19; Wagner in MünchKomm, BGB, Bd. 5, 2013, §823 Rn. 242; Simitis in Simitis, BDSG, § 7 Rn. 59; Neunhoeffer, 201.

227 BGH, BGHZ 80, 311, 319; BGH, BGHZ 89, 218, 226; BGH, BGHZ 91, 233, 237f.; BGH, NJW 1986, 2505, 2506f.; Neunhoeffer, 201. 
bestimmte Konstellation nicht abschließend geregelt hat. ${ }^{228}$ Der Ersatz immaterieller Schäden ist nach der überwiegenden Auffassung genau der Fall. ${ }^{229}$ Ein Anspruch auf immateriellen Schadensersatz wegen Verletzung des allgemeinen Persönlichkeitsrechts kann nach $\$ 823$ Abs. 1 BGB in Verbindung mit dem Verfassungsrechtlichen Schutzauftrag aus Art. 1 und 2 Abs. 1 GG verlangt werden. ${ }^{230}$

\section{Der persönliche Datenschutz in China}

\section{Die Geschichte und der gegenwärtige Stand der Legislative vom persönlichen Datenschutz in China}

Die Legislative vom persönlichen Datenschutz hat keine lange Geschichte in China. Es gibt im chinesischen Verfassungsgesetz keine direkte Regelung über das Recht auf informationelle Selbstbestimmung oder persönlichen Datenschutz. Nach der herrschende Meinung liegt der Grundgedanke über persönlichen Datenschutz in $\$ \$ 38,39$ und $\$ 40$ chinesisches Verfassungsgesetzes.

$\$ 38$ Verfassungsgesetz ist eine allgemeine Regelung über den Schutz der Menschenwürde vor Rechtsverletzung. \$39 Verfassungsgesetz schützt das Wohnhaus vor rechtswidrigem Eintritt, rechtswidriger Durchsuchung oder anderer Art von Eingriffen. $\$ 40$ Verfassungsgesetz ist eine allgemeine Regelung über den Schutz der Kommunikationsfreiheit und des Kommunikationsgeheimnisses. Aber weil es in China kein funktionierendes Auslegungs- und Durchsetzungssystem vom Verfassungsrecht gibt, blieb die Diskussion über das Recht auf informationelle Selbstbestimmung und den persönlichen Datenschutz für eine lange Zeit nur auf theoretischem Niveau. ${ }^{231}$

Mit der schnellen Entwicklung der Informationstechnik wird eine Gesetzgebung über persönlichen Datenschutz immer notwendiger. Das Informationsbüro des chinesischen Staatsrates hat im Jahr 2003 entschieden, mit dem Gesetzgebungsprozess über persönlichen Datenschutz anzufangen. Im Jahr 2005 hat die Forschungsgruppe über persönlichen Datenschutz, die von Experten aus dem juristische Forschungsinstitute der Chinesische Akademie der Sozialwissenschaften gegründet wurde, die nach Auftrag entworfene Version „Persönliches

228 Hager in Staudinger, BGB, 1999, $\$ 823$ Rn. C 173; Simitis in Simitis, BDSG, § 7 Rn. 60; Neunhoeffer, 202.

229 Gola/Schomerus in Gola/Schomerus, BDSG $₫ 7$, Rn. 19; Simitis in Simitis, BDSG, $\S 7$ Rn. 64; Neunhoeffer, 202.

230 Gola/Schomerus in Gola/Schomerus, BDSG $₫ 7$, Rn. 19; Simitis in Simitis, BDSG, $\S 7$ Rn. 60; Neunhoeffer, 203.

231 Yao Yuerong, Political Science and Law 2012, No. 4, 72, 82. 
Datenschutzgesetz - Vorschlagsversion von den Experten“ und den legislativen Forschungsbericht dem Staatsrat abgegeben. Im Jahr 2008 wurde ein Entwurf vom „Persönlichen Datenschutzgesetz“ dem Staatsrat abgegeben. ${ }^{232}$ Aber seitdem ist der Gesetzgebungsprozess stehengeblieben. Die Interaktion zwischen den juristischen Wissenschaftlern und den Gesetzgebern ist gestoppt. Über die Vorschläge von den Juristen auf die Beschleunigung des Gesetzgebungsprozesses hatte der Gesetzgeber nicht aktiv respondiert. ${ }^{233}$

Es herrscht die Meinung, dass der Grund des langsamen Gesetzgebungsprozesses daran liegt, dass ein persönliches Datenschutzgesetz zu viele Verwaltungsabteilungen betrifft, ${ }^{234}$ wie z.B. die Abteilung von Telekommunikation, Industrie und Kommerz, Bankwesen, Justiz, öffentliche Sicherheit, Staatsadministration. ${ }^{235}$ Die Schwierigkeit der Zusammenarbeit und Kooperation zwischen den Abteilungen ist der Hauptgrund für den langsamen Gesetzgebungsprozess. ${ }^{236}$

Ab Ende des Jahres 2011 sind einige einzelne Regelungen über bestimmte Themen aus dem Bereich des persönlichen Datenschutzes veröffentlicht worden. Dazu gehörten „Einige Regelungen zur Regulierung des Markts von Informationsdienst im Internet", die von dem Ministerium von Industrie und Informationstechnik am 7.12.2011 erlassen wurde und ab 15.3.2012 in Kraft getreten ist. Diese Regelung behandelt die Rechte und Pflichten des ISP im Prozess der Erhebung persönlicher Daten von ihren Kunden. Eine andere Regelung ist die „Entscheidung zur Verstärkung des Informationsschutzes im Internet“ (im folgenden „Informationsschutz-Entscheidung“), die von dem Ständigen Komitee des Nationalen Volkskongresses am 28. Dez. 2012 erlassen wurde und in Kraft getreten ist. Aufgrund dieser Entscheidung wird die digitale Information, mit der man eine Person identifizieren kann oder die der persönlichen Privatsphäre zuzurechnen ist, vom Staat geschützt. Aufgrund $₫ 8$ „Informationsschutz-Entscheidung“ hat der Rechtsinhaber das Recht gegen

232 China Youth Daily, http://dailynews.sina.com/gb/chn/chnpolitics/sinacn/2012 0522/13033411440.html (besucht am 04.04.2015).

233 China Youth Daily, http://dailynews.sina.com/gb/chn/chnpolitics/sinacn/2012 0522/13033411440.html (besucht am 04.04.2015).

234 China Youth Daily, http://dailynews.sina.com/gb/chn/chnpolitics/sinacn/2012 0522/13033411440.html (besucht am 04.04.2015).

235 China Youth Daily, http://dailynews.sina.com/gb/chn/chnpolitics/sinacn/2012 0522/13033411440.html (besucht am 04.04.2015).

236 China Youth Daily, http://dailynews.sina.com/gb/chn/chnpolitics/sinacn/2012 0522/13033411440.html (besucht am 04.04.2015). 
den ISP, die Löschung der oben genannten Information zu fordern, oder die Vornahme anderer Maßnahmen zu verlangen, um ein rechtswidriges Verhalten gegen ihn zu stoppen.

Aber die oben genannten Regelungen stehen ganz unten in der Normenpyramide. Die Anzahl der Paragraphen ist gering. Die Inhalte sind meistens Prinzipien und schlecht durchsetzbar. Deswegen ist es immer noch eine nötige Aufgabe, ein persönliches Datenschutzgesetz zu verabschieden.

Übrigens ist das Recht auf informationelle Selbstbestimmung in China noch nicht anerkannt. Es gibt bisher noch keinen passenden Begriff, um das Recht des Betroffenen auf seine persönlichen Daten zu beschreiben. In der "Informationsschutz-Entscheidung“ wird das Recht auf informationelle Selbstbestimmung als „ein rechtmäßiges Rechtsgut des Betroffenen“ genannt. In der Praxis ist es häufig, dass das Recht des Betroffenen im Fall von Verletzung des Rechts auf informationelle Selbstbestimmung durch den Schutz des Rechts auf Privatsphäre verwirklicht wird. ${ }^{237}$

\section{Die Anwendbarkeit der "Informationsschutz-Entscheidung“ auf Menschenfleischsuche}

Als die wichtigste Regelung über persönlichen Datenschutz im Internet hat „Informationsschutz-Entscheidung “ die Tür auch für den persönlichen Datenschutz während Menschenfleischsuche geöffnet. Auf Grund $\$ 1$ „InformationsschutzEntscheidung" ist das Recht des Betroffenen auf seine persönlichen Daten nicht nur vor der rechtswidrigen Erhebung und Übermittlung von juristischen Personen und Organisationen, sondern auch vor der von natürlichen Personen geschützt. Anders als in Deutschland ist der Verwendungszweck der persönlichen Daten in der „Informationsschutz-Entscheidung“ nicht betont. Die persönliche oder familiäre Benutzung persönlicher Daten wie im Fall von Menschenfleischsuche wird auch von der „Informationsschutz-Entscheidung“ geregelt.

\section{Die Eingrenzung der zu schützenden persönlichen Daten}

Gemäß $₫ 1$ der „Informationsschutz-Entscheidung“ ist der Schutzgegenstand der Entscheidung nur die digitale Information, mit der man eine Person identifizieren kann oder die der persönlichen Privatsphäre zuzuordnen ist. Aus diesem Paragraph kann man zwei Schlussfolgerungen treffen.

237 Nantong Mitteleres Volksgericht, Urt. v. 15.08.2011 - (2011) tong zhong min zhong zi di 0952 hao. 
Erstens sind nur die persönlichen Daten geschützt, die Identifikationsfunktion haben. Es herrscht die gleiche Meinung in der Literatur. ${ }^{238}$ Das ist ähnlich der Bestimmbarkeit des Betroffenen i.S.v. $\$ 3$ Abs. 1 BDSG. Meiner Meinung nach soll die Identifizierbarkeit breit ausgelegt werden. Der Grund liegt darin, dass jede im Internet veröffentlichte persönliche Information als Hinweis benutzt werden könnte, um weitere Informationen des Betroffenen zu bekommen. Und jede einzelne persönliche Information im Internet kann durch Informationstechnik kombiniert werden und zum Identifizieren einer Person hilfreich sein. ${ }^{239}$ Jede personenbezogene Information hat mehr oder weniger Identifikationsfunktion. Sie verdient deswegen den gleichen Schutz. ${ }^{240}$

Zweitens sind die die persönlichen Privatsphären betreffenden Daten auch Schutzgegenstand der „Informationsschutz-Entscheidung“. Für solche Daten ist eine Identifikationsfunktion nicht vorausgesetzt. Durch diese Regelung hat die „Informationsschutz-Entscheidung“ das Verhältnis zwischen Datenschutz und Schutz der Privatsphäre deutlich geklärt. Digitalisierte Privatsphäre kann in China auch durch Datenschutz geschützt werden.

238 Wang Liming, Science of Law 2013, 166, 172.

239 Qi Aimin, Saving the personality from the information society, S. 28-30.

240 Zhang Zhuochen, Science \& Technology Information 2009, 770. 


\section{$\S 5$ Der Schutz des Rechts auf Privatsphäre}

\section{Der Schutz des Rechts auf Privatsphäre in Deutschland}

\section{Schutz der Privatsphäre durch Schutz allgemeiner Persönlichkeitsrechte}

Es gibt in deutscher Sprache keinen genau passenden Begriff wie „the right to privacy" auf English, der im Jahr 1890 von Samuel Warren und Louis Brandeis erfunden wurde. ${ }^{241}$ Es gibt allenfalls einen ähnlichen Begriff mit „das Recht auf Privatsphäre“. Das Wort Privatsphäre ist jedoch nach der unten genannten Sphärentheorie mehrdeutig. Das Wort „Privacy“ bedeutet eigentlich Intimsphäre, Privatsphäre in engeren Sinn und manchmal einen Teil von der Sozialsphäre. In dieser Arbeit wird das Wort Privatsphäre meistens als Privacy verwendet. Auf Bedarf wird es auch i.S.v. Sphärentheorie, also im engen Sinne benutzt.

Das Recht auf Privatsphäre ist in Deutschland nicht vom Gesetz unmittelbar geregelt. Dessen Schutz wird durch den Schutz des allgemeinen Persönlichkeitsrechts (Art 1, Art 2 GG) verwirklicht. Das allgemeine Persönlichkeitsrecht beinhaltet nach einer Rechtsprechung das Recht, „in gewählter Anonymität zu bleiben und die eigene Person nicht in der Öffentlichkeit dargestellt zu sehen "242, was genau der Fall vom Recht der Zielperson während Menschenfleischsuche ist. Diskretionsschutz ist ein wichtiger Teil vom Persönlichkeitsschutz. ${ }^{243}$

Im Bereich vom allgemeinen Persönlichkeitsrecht hat das BVerfG im Jahr 1957 die Sphärentheorie entwickelt, ${ }^{244}$ die zwar mehrfach auf Kritik gestoßen ist, ${ }^{245}$ aber für den Schutz der Privatsphäre immer eine Rolle spielt und deswegen zu erwähnen ist.

\section{Sphärentheorie}

Aufgrund der Sphärentheorie ist der persönliche Lebensbereich in drei Sphären gegliedert, nämlich Intimsphäre, Privatsphäre und Sozialsphäre.

241 Warren/Brandeis, Harvard Law Review 1890, S. $193 \mathrm{ff}$.

242 KG, GRUR-RR 2007, 247.

243 Nink in Spindler/Schuster, BGB $\$ 823$, Rn. 21.

244 BVerfG, BVerfGE 6, 32, 41 f. = NJW 1957, 297, 298; BVerfG, BVerfGE 38, 312, $320=$ NJW 1975, 588 .

245 Kannowski in Staudinger, BGB Vorbemerkungen zu $₫ 1$, Rn. 25; Nink in Spindler/ Schuster, BGB $\$ 823$, Rn. 22. 


\section{a) Intimsphäre}

„Die Intimsphäre umfasst den letzten unantastbaren Bereich menschlicher Freiheit. “246 Als Intimsphäre zählen insbesondere Vorgänge aus dem Sexualbereich $^{247}$, Tagebüchern ${ }^{248}$, Krankheit und Tod ${ }^{249}$, persönliche Vorlieben und Gewohnheiten ${ }^{250}$, religiöse Anschauungen ${ }^{251}$ etc.

Die Intimsphäre wird absolut geschützt ${ }^{252}$. Das heißt, eine Offenlegung der Intimsphäre ohne bezügliche Einwilligung ist stets unzulässig. ${ }^{253}$ Ein gerechtfertigtes gegenseitiges allgemeines Interesse ist grundsätzlich nicht $\mathrm{zu}$ begründen. ${ }^{254}$ Eine Interessenabwägung in diesem Fall ist daher nicht möglich. ${ }^{255}$

\section{b) Privatsphäre}

Die Privatsphäre umfasst den engen Bereich der höchstpersönlichen Lebensführung. ${ }^{256}$ Dazu gehört hauptsächlich häuslicher und familiärer Bereich ${ }^{257}$, aber nicht beschränkt. ${ }^{258}$ Die Offenlegung der persönlichen Information aus diesem Bereich würde als „unschicklich" bezeichnet und als peinlich empfunden werden, oder nachteilige Reaktionen in der Öffentlichkeit auslösen. ${ }^{259}$ Deswegen würde eine öffentliche Erörterung über Privatsphäre grundsätzlich als unzulässig angesehen. ${ }^{260}$

Aber anders als Intimsphäre verdient Privatsphäre keinen absoluten Schutz. Eine Interessenabwägung zwischen dem Interesse des Betroffenen am

246 Nink in Spindler/Schuster, BGB $\$ 823$, Rn. 23.

247 Vgl. z. B. OLG Karlsruhe, NJW 2006, 617; BVerfGE 96, 56, 61.

248 Nink in Spindler/Schuster, BGB $\$ 823$, Rn. 23; ist jedoch umstritten, vgl. Hager in Staudinger, Eckpfeiler des Zivilrechts, T. Das Recht der unerlaubten Handlungen, Rn. 344; siehe auch BVerfG, BVerfGE 80, 374 = NJW 1990, 563 = NStZ 1990, 397.

249 Gounalakis/Rhode, Rn. 196; Nink in Spindler/Schuster, BGB $₫ 823$, Rn. 23.

250 Ballhausen/Roggenkamp, K\&R 2008, 403, 405.

251 Ballhausen/Roggenkamp, K\&R 2008, 403, 405.

252 Vgl. BVerfG, BVerfGE 96, 56, 61 = NJW 1997, 1769; OLG Karlsruhe, NJW 2006, 617.

253 Ballhausen/Roggenkamp, K\&R 2008, 403, 405.

254 BVerfGE 34, 238, 244; Nink in Spindler/Schuster, BGB \$ 823, Rn. 23.

255 BVerfG, NJW 1990, 563, 565; BGH, NJW 1985, 1617, 1618; Nink in Spindler/ Schuster, BGB $\$ 823$, Rn. 23.

256 BVerfGE 65, 1, 14 f.; Nink in Spindler/Schuster, BGB $\$ 823$, Rn. 24.

257 Ballhausen/Roggenkamp, K\&R 2008, 403, 405.

258 Nink in Spindler/Schuster, BGB $\$ 823$, Rn. 24.

259 Ballhausen/Roggenkamp, K\&R 2008, 403, 405.

260 Ballhausen/Roggenkamp, K\&R 2008, 403, 405. 
Persönlichkeitsschutz und den Informationsinteressen der Öffentlichkeit, sowie anderen gegenüberstehenden grundrechtlichen Interessen ist stets möglich. ${ }^{261}$

$\mathrm{Zu}$ der Privatsphäre gehört nach den Rechtsprechungen z.B. die Privatanschrift ${ }^{262}$ und der Gesundheitszustand eines Menschen ${ }^{263}$, Bewertungen der „Attraktivität" oder des allgemeinen „Charakters“ einer Person ${ }^{264}$.

\section{c) Sozialsphäre}

Intimsphäre und Privatsphäre ausgeschlossen, gehören die restlichen persönlichen Bereiche zur Sozialsphäre. Darunter fallen insbesondere berufliche, gewerbliche, sowie politische Betätigungen. ${ }^{265}$ Anders als Privatsphäre ist die Veröffentlichung der Informationen aus diesem Bereich regelmäßig erlaubt, weil hier das Informationsinteresse der Öffentlichkeit häufig von großer Bedeutung ist. ${ }^{266}$ Ein Verbot der Offenlegung persönlicher Information aus der Sozialsphäre ist dementsprechend ein Sonderfall.

Der Grundgedanke liegt darin, dass der Betroffene durch den Eintritt in die Gemeinschaft bzw. durch die Kommunikation und Interaktion mit anderen Bürgern die persönliche Sphäre der Mitmenschen und die Belange der Gemeinschaft berührt hat. ${ }^{267}$ Das Informationsinteresse der Mitmenschen bzw. der Gemeinschaft an dem Betroffenen ist daher begründet. Ein Zugriff zur Sozialsphäre ist zulässig, solange sich daraus keine schwerwiegende Auswirkung auf das Persönlichkeitsrecht des Betroffenen entwickelt, „so etwa dann, wenn eine Stigmatisierung, soziale Ausgrenzung oder Prangerwirkung zu besorgen sind““ ${ }^{268}$

Jedoch ist die Unterscheidung der drei Sphären nicht unveränderlich. Die Situation in jedem einzelnen Fall spielt immer eine Rolle. Die „Spick-mich“Entscheidung vom BGH hat ein Beispiel gezeigt. Hier ging es um die Bewertungskategorien gegen die Lehrer und Lehrerinnen wie „cool und witzig“, „beliebt“ oder „menschlich“, die grundsätzlich zur Privatsphäre zu zählen sind. ${ }^{269}$ Aber der BGH meinte, dass die Lehrer und Lehrerinnen Rollenvorbilder für

261 EGMR, NJW 1999, 1315, 1316; BVerfGE 35, 202, 221; BVerfG, NJW 2000, 2189; BVerfG, AfP 2001, 212, 214 f.; Nink in Spindler/Schuster, BGB $\$ 823$, Rn. 24.

262 OLG Hamburg, ZUM 2008, 66.

263 BGH, NJW 1996, 984, 985; vgl. BVerfGE 32, 373, 379f.= NJW 1972, 1123.

264 Ballhausen/Roggenkamp, K\&R 2008, 403, 405; Vgl. OLG Köln, K\&R 2008, 40, 43.

265 Nink in Spindler/Schuster, BGB \$ 823, Rn. 25.

266 BGH, AfP 1995, 404, 407; Nink in Spindler/Schuster, BGB $\$ 823$, Rn. 25.

267 Vgl. BGH, NJW-RR 2007, 619, 620.

268 Vgl. BGH, NJW-RR 2007, 619, 620.

269 Gounalakis/Klein, NJW 2010, 566, 569. 
ihre Schüler sind, und im Schultag bewusst über ihr Verhalten auch persönliche Eigenschaften preisgeben. ${ }^{270}$ Damit werden die persönlichen Eigenschaften der Lehrer und Lehrerinnen wegen der Besonderheiten im schulischen Wirkungskreis zur Sozialsphäre gezählt. ${ }^{271}$

Die Anwendung der Sphärentheorie muss nach einzelnen Konfliktsituationen näher Konkretisiert werden. ${ }^{272}$ Die Grenzen der drei Sphären könnten in einzelnen Fällen abweichend sein. ${ }^{273}$ Genau deswegen hat die Sphärentheorie viel Kritik bekommen. ${ }^{274}$ Umfangreiche Rechtsprechung und Literatur sind entwickelt worden, um die Interesseabwägung zwischen Persönlichkeitsrecht und anderen Rechten in unterschiedlichen Situationen zu konkretisieren. ${ }^{275}$ Es gibt sogar den Vorschlag, auch für den Fall, der der Intimsphäre bezüglich ist, eine Interesseabwägung durchzuführen. ${ }^{276}$ Eine Forschung nach charakteristischen Konflikten und Entscheidungstendenzen ist die einzige Möglichkeit, um das Problem vom Schutz der Persönlichkeitsrechte bzw. des Rechts auf Privatsphäre zu erläutern.

\section{Der Schutz des Rechts auf Privatsphäre in China}

\section{Die Rechtsentwicklung über das Recht auf Privatsphäre in China}

Vor dem Erlass des chinesischen „Delikthaftungsgesetzes“ im Dez. 2009 gab es im chinesischen zivilrechtlichen System keine direkte Regelung über das Recht auf Privatsphäre. Der Schutz des Rechts auf Privatsphäre war ein Unterthema vom Ehrenschutz.

Die Hauptregelung über Ehrenschutz im zivilrechtlichen Bereich besteht in $\$ 101$ AGZR vom 01.01.1987. Aufgrund dieses Paragraphen haben die natürliche Person und die juristische Person das Recht auf ihre Ehre; ihre Ehre darf nicht durch Beleidigung, Verleumdung oder andere Weise verletzt werden. Wenn die

270 Gounalakis/Klein, NJW 2010, 566, 569.

271 Gounalakis/Klein, NJW 2010, 566, 569.

272 Kannowski in Staudinger, BGB Vorbemerkungen zu $₫ 1$, Rn. 25.

273 Vgl. Hager in Staudinger, Eckpfeiler des Zivilrechts, T. Das Recht der unerlaubten Handlungen, Rn. 344 und Nink in Spindler/Schuster, BGB \$ 823, Rn. 23; vgl. auch BVerfG, NStZ 1990, 397.

274 Nink in Spindler/Schuster, BGB $\$ 823$, Rn. 22; Rixecker in Münchener Kommentar zum BGB, Anhang zu $\$ 12$, Rn. 9 f.; Wöfl, NVwZ 2002, 49, 50 f.; Degenhart, JuS 1992, 364.

275 Kannowski in Staudinger, BGB Vorbemerkungen zu $\$ 1$, Rn. 25.

276 Hager in Staudinger, Eckpfeiler des Zivilrechts, T. Das Recht der unerlaubten Handlungen, Rn. 344. 
Ehre verletzt ist, hat der Rechtsinhaber aufgrund \$120 AGZR den Anspruch auf Unterlassung des rechtswidrigen Verhaltens, Wiedergutmachung der Ehre, Beseitigung der durch die Rechtsverletzung entstehenden Einwirkung sowie offizielle Entschuldigung und Schadenersatz.

Als Unterthema des Ehrenschutzes wurde der Schutz des Rechts auf Privatsphäre vom chinesischen Obersten Volksgerichtshof im Jahr 1988 durch die OVG-AGZR-Ansichten festgestellt. Im $\$ 140$ Abs. 1 OVG-AGZR-Ansichten ist das Wort „Privatsphäre“ das erste Mal genannt: wird die Privatsphäre einer Person schriftlich, mündlich oder in anderer Form verbreitet, und ist dadurch eine gewisse Auswirkung entstanden, soll die Verbreitung der Privatsphäre als Ehrverletzung identifiziert werden. Für die Ehrverletzung kann der Verletzte gemäß dem Standard des $\$ 150$ OVG-AGZR-Ansichten Schadenersatz verlangen.

Im Jahr 1993 hat der chinesische Oberste Volksgerichtshof im $\$ 7$ seiner „Antwort zu einigen Fragen hinsichtlich der Beurteilung der Fälle über Ehrverletzung" eine deutlichere Erklärung über das Verhältnis zwischen dem Schutz der Privatsphäre und dem Ehrenschutz gegeben: das Verhalten, die der Privatsphäre zuzuordnenden Unterlagen des anderen ohne dessen Einwilligung eigenmächtig zu veröffentlichen, oder die Privatsphäre des anderen in schriftlicher oder mündlicher Form zu verbreiten, soll als Ehrverletzung behandelt werden, wenn die Ehre des anderen durch das Verhalten beschädigt ist. Auch hier ist die Privatsphäre nur als ein Rechtsgut unter dem Thema Ehrenschutz geschützt. Das Recht auf Privatsphäre blieb von den chinesischen Rechtsnormen ungeregelt.

Das Recht auf Privatsphäre ist erst ab 2009 durch das Delikthaftungsgesetz als ein Recht offiziell anerkannt. Aufgrund $\$ 2$ Abs. 1 Delikthaftungsgesetz soll derjenige, der ein zivilrechtliches Interesse verletzt hat, nach diesem Gesetz haften. Gemäß $₫ 2$ Abs. 2 Delikthaftungsgesetz gehören das Recht auf Privatsphäre mit dem Recht am eigenen Bild, dem Recht der persönlichen Ehre und den anderen Rechten zusammen zu den geschützten zivilrechtlichen Interessen.

\section{Die Eingrenzung der Privatsphäre}

\section{a) Die Unbestimmbarkeit des Inhalts von Privatsphäre}

Die Unbestimmbarkeit des Inhalts von Privatsphäre liegt erstens daran, dass sich der Inhalt der Privatsphäre zwischen unterschiedlichen Personen unterscheidet. Genau wie die Kritik über die Sphärentheorie in Deutschland, dass die Sphären für verschiedene Menschen von unterschiedlichem Inhalt sein können, ${ }^{277}$

277 Nink in Spindler/Schuster, BGB $\$ 823$, Rn. 22. 
besteht in der chinesischen Literatur auch die Diskussion, dass der Inhalt der Privatsphäre für unterschiedlichen Menschen unterschiedlich ist.

Der Grund, warum sich der Inhalt der Privatsphäre zwischen unterschiedlichen Personen unterscheidet, liegt nach der chinesischen Literatur darin, dass der eigene Wille einer Person auf den Inhalt der Privatsphäre eine Wirkung hat. ${ }^{278}$ Die von einer Person als Privatsphäre angesehene Information könnte von einem anderen nicht als Privatsphäre angesehen werden, weil er mit Absicht solche Information veröffentlicht hat. Wegen der unterschiedlichen Menge und Arten von privaten Informationen, die eine Person absichtlich veröffentlicht hat, ist der Inhalt der Privatsphäre für jede Person unterschiedlich.

Die Unbestimmbarkeit des Inhalts von Privatsphäre liegt weiterhin daran, dass der Inhalt der Privatsphäre sich mit der Zeit, der Sozialentwicklung und dem Moralhintergrund ändert. ${ }^{279}$ Der Inhalt, der in einem Zeitalter oder in einem Land nicht zur Privatsphäre gehört, könnte in einem anderen Land oder in einem anderen Zeitalter zur Privatsphäre gehören.

\section{b) Die Grenze der Privatsphäre}

Obwohl der genaue Inhalt der Privatsphäre in jedem einzelnen Fall unterschiedlich ist, ist eine allgemeine Eingrenzung von Privatsphäre für die weitere Forschung nötig.

Über die Grenze der Privatsphäre gibt es in China unterschiedliche Meinungen, ${ }^{280}$ Einige Juristen versuchen durch Aufzählung der zur Privatsphäre gehörenden Informationen die Grenze der Privatsphäre darzustellen. ${ }^{281}$ Gemäß der Entscheidungspraxis und den herrschenden Meinungen sind die folgenden Informationen als zur Privatsphäre zugehörig festgelegt: Kommunikationshistorie von $\mathrm{MSN}^{282}$, Telefonnummer ${ }^{283}$, Anschriften ${ }^{284}$, Informationen über ärztliche

278 Li Xiufen, Contemporary Law Review 2004, 98, 99.

279 Li Jun/Zhang Jun, People's Judicature (case) 2008, 24, 27.

280 Wang Liming, Science of Law 2013, 166, 172; Zhang Xinbao, Legal protection of the right to privacy, S. 17; Wang Liming/Yang Lixin, S. 415.

281 Zhang Xinbao, Hu lian wang shang de qin quan wen ti yan jiu, S. 189; Li Jun/Zhang Jun, People's Judicature (case) 2008, 24, 27; Dai Yingjie, Journal of Zhejiang Institute of Media and Communications 2011, 37, 39.

282 Vgl. Shanghai Erstes Mitteleres Volksgericht, Urt. v. 2009 - (2009) hu yi zhong min yi (min) zhong zi di 1431 hao.

283 Vgl. Kunming Mitteleres Volksgericht, Urt. v. 2004 - (2004) kun min er zhong zi di 785 hao.

284 Vgl. Baoan Unteres Volksgericht, Urt. v. 2010 - (2010) shen bao fa min yi chu zi di 1034 hao. 
Behandlung, ${ }^{285}$ physiologische Behinderungen ${ }^{286}$, Nacktbilder ${ }^{287}$, sexuelles Leben und sexuelle Beziehung ${ }^{288}$, Antrag der DNA-Identifikation zwischen Eltern und Kindern ${ }^{289}$, das Erlebnis eines Vergewaltigungsopfers ${ }^{290}$, die Namen und Alter der Opfer eines Verbrechens ${ }^{291}$. Außerdem gehören persönliche DNAInformation, Information der gesundheitlichen Überwachung und persönliche Vorstrafen gemäß $\$ 12$ der aktuellste „Regelung vom Obersten Volksgericht über einigen Fragen hinsichtlich der anzuwendenden Gesetzen bei der Beurteilung von zivilrechtlichen Streitigkeiten wegen Verletzung der persönlichen Rechte und Interesse durch Internet ${ }^{\text {"292 }}$ auch zur Privatsphäre.

Außer den persönlichen Informationen gehören zur Privatsphäre nach der herrschenden Meinung noch die privaten Aktivitäten, der private Raum und die Gelassenheit des Privatlebens, die man für sich behalten will. ${ }^{293} 294$ Diese Eingrenzung der Privatsphäre ist von der Justizpraxis akzeptiert und in den Entscheidungen zitiert. ${ }^{295}$

Unter dem Thema der Eingrenzung der Privatsphäre ist der Begriff ,illegale Privatsphäre" von einigen chinesischen Juristen eingebracht worden. Illegale Privatsphäre ist ihren Meinungen nach die Privatsphäre, deren Inhalt rechtsoder moralwidrig ist, ${ }^{296}$ wie z.B. eine Vorliebe, eigene lebende Haustiere zu töten

285 Vgl. Qianjiang Unteres Volksgericht, Urt. v. 2008 - (2008) qian fa min chu zi di 284 hao.

286 Vgl. Lixia Unteres Volksgericht, Urt. v. 1999 - (1999) li min chu zi di 276 hao.

287 Vgl. Shanghai Zweites Mitteleres Volksgericht, Urt. v. 2009 - (2009) hu er zhong min yi (min) zhong zi di 451 hao.

288 Vgl. Qitai Unteres Volksgericht, Urt. v. 2006 - (2006) qi min yi chu zi di 524 hao; Changji Mitteleres Volksgericht, Urt. v. 2006 - (2006) chang zhong min yi zhong zi di 826 hao.

289 Vgl. Weidong Unteres Volksgericht, Urt. v. 2010 - (2010) wei min zai chu zi di 1 hao.

290 Vgl. Tongzhou Unteres Volksgericht, Urt. v. 2002 - (2002) tong min chu zi di 1113 hao; Tongzhou Mitteleres Volksgericht, Urt. v. 2002 - (2002) tong zhong min yi zhong zi di 1470 hao.

291 Vgl. Qitai Unteres Volksgericht, Urt. v. 2006 - (2006) qi min yi chu zi di 524 hao; Changji Mitteleres Volksgericht, Urt. v. 2006 - (2006) chang zhong min yi zhong zi di 826 hao.

292 Erlassen am 23.6.2014, gültig ab 10.10.2014.

293 Yang Lixin, A propositional version with explaination for tort liability law draft of China, S. 137.

294 Dai Yingjie, Journal of Zhejiang Institute of Media and Communications 2011, 37, 39.

295 Zhang Jianwen, Journal of Henan University of Economics and Law 2012, No. 2, 95, 96-97.

296 Liang Huixing/Liao Xinzhong, People’s Judicature 2003, 42, $43 f$. 
oder tief in der Nacht auf einer leeren Straße ohne Rücksicht auf Ampeln zu fahren. Sie glauben, dass die illegale Privatsphäre prinzipiell nicht als Privatsphäre angesehen werden soll, und deswegen nicht schutzwürdig ist. ${ }^{297}$ Das bedeutet im Fall der Menschenfleischsuche, dass jeder Internetnutzer die illegale Privatsphäre im Internet offenlegen darf.

Meiner Meinung nach soll auch die illegale Privatsphäre als normale Privatsphäre behandelt werden. Erstens ist nicht jedes illegale Verhalten strafbar. Zweitens müssen für strafbares Verhalten bestimmte Verfahren befolgt werden. Drittens ist es häufig undeutlich, ob eine Privatsphäre illegal ist; dem Internetnutzer sollte letztlich nicht die Entscheidung zustehen, eine Entscheidung darüber zu treffen, was legal bzw. illegal ist. Es ist immer notwendig, durch eine Abwägung in jedem einzelnen Fall zu untersuchen, ob gegenüber der Privatsphäre ein überwiegendes öffentliches Interesse steht. ${ }^{298}$ Die Beurteilung, ob eine Privatsphäre illegal ist, ist in der Tat schon Teil der Interessenabwägung. Für die illegale Privatsphäre ist das öffentliche Interesse häufig überwiegend. Aber es kann auch sein, dass der Rechtsinhaber ein überwiegend geschütztes Interesse an seinem rechtswidrigen Verhalten hat. Das ist z.B. der Fall, wenn ein Minderjähriger ein rechtswidriges Verhalten begangen hat.

Im Mai 2013 hat ein chinesischer Tourist während seines Besuchs eines 3000 Jahre alten Tempels in Ägypten auf einem Relief gesehen, dass ein Chinese darauf auf Chinesisch „Ding Jinhao war mal hier“ eingraviert hatte. Der Tourist hat dann eine Menschenfleischsuche nach Ding Jinhao ausgelöst, der, wie rausgefunden wurde, ein minderjähriger Schüler ist. Dieses Ereignis wurde von vielen Medien berichtet. ${ }^{299}$ Das Leben von Ding Jinhao hat sich seit der Bekanntmachung seines Verhaltens großartig verändert. Er wurde in vielen Interviews von verschiedenen Medien damit konfrontiert und musste unter den Vorwurf vom Internet und von seinen Kommilitonen leiden. Für sein Verhalten als Minderjährige ist die „Strafe“ meiner Meinung nach viel zu übertrieben. Gemäß $₫ 58$ des Gesetzes zum Schutz der Minderjährigen darf der Name, die Adresse, die Bilder sowie die andere persönliche Information der Minderjährigen nicht veröffentlicht werden, wenn ein von ihm begangenes Verbrechen in den Nachrichten, im Internet, durch Fernseh- und Filmprogramm oder öffentlichen Publikationen berichtet wird. Das öffentliche Interesse an einem kriminellen Verhalten des Minderjährigen steht sogar unter dem Interesse des Jugendschutzes, ganz zu

297 Liang Huixing/Liao Xinzhong, People's Judicature 2003, 42, 43f.

298 Vgl. Zhou Youjun, Netinfo Security 2009, 49, 50.

299 Siehe: http://en.wikipedia.org/wiki/Ding_Jinhao_engraving_scandal (besucht am 04.04.2015). 
schweigen von dem was Ding Jinhao gemacht hat. Das Interesse, die Privatsphäre der Schüler zu schützen, ist offensichtlich überwiegend, obwohl er rechtswidriges Verhalten begangen hat.

Das Interesse am Jugendschutz ist nur ein Beispiel. An einem rechtswidrigen Verhalten könnte noch anderes Interesse liegen, das man nicht so leicht einfach unter der Priorität des öffentlichen Interesses stellen kann. Die Schutzwürdigkeit illegaler Privatsphäre soll deswegen ebenso wie die allgemeine Privatsphäre über eine Interessenabwägung beurteilt und gewertet werden. Eine besondere Handlung an der illegalen Privatsphäre ist meiner Meinung nach nicht nötig.

\section{Das Recht auf Privatsphäre}

Für die Definition des Rechts auf Privatsphäre gibt es in der chinesischen Literatur unterschiedliche Meinungen. Die meisten Juristen definieren das Recht auf Privatsphäre als ein Persönlichkeitsrecht. ${ }^{300}$ Es gibt auch den Versuch, das Recht auf Privatsphäre als ein Grundrecht zu definieren. ${ }^{301}$

Die Kommission über legislative Angelegenheiten vom ständigen Ausschuss des Nationalen Volkskongresses und das legislative Büro des Staatsrates der Volksrepublik China haben bei der inoffiziellen Erklärung des Delikthaftungsgesetzes eine Definition vom Recht auf Privatsphäre gegeben: das Recht auf Privatsphäre ist ein Persönlichkeitsrecht der natürlichen Person, auf Grund dessen sie über ihre öffentlichen Interessen bzw. Gruppeinteressen irrelevante persönliche Informationen, ihre privaten Aktivitäten, ihre privaten Sphären frei verfügen kann. ${ }^{302}$ Diese Definition wurde vor dem Inkrafttreten des Delikthaftungsgesetzes schon in der Entscheidungspraxis umfangreich verwendet; ${ }^{303}$ sie repräsentiert auch die herrschende Meinung in der Literatur. ${ }^{304}$

Aufgrund dieser Definition kann man zwei Schlussfolgerungen nachvollziehen:

Erstens ist das Recht auf informationelle Selbstbestimmung im Recht auf Privatsphäre eingeschlossen. Wie oben gesagt wurde, ist das Recht auf informationelle Selbstbestimmung in China noch nicht anerkannt. Die persönlichen Daten

300 Yang Lixin, Monograph on personal right, S. 336; Zhang Xinbao, Legal protection of the right to privacy, S. 17.

301 Wang Xiuzhe, S. 35.

302 http://www.civillaw.com.cn/article/default.asp?id=55617 (besucht am 04.04.2015).

303 Zhongshan Mitteleres Volksgericht, Urt. v. 25.11.2005 - (2005) zhong zhong fa min yi zhong zi di 1003 hao; vgl. Zhang Jianwen, Journal of Henan University of Economics and Law 2012, No. 2, 95, 96-97.

304 Yang Lixin, Monograph on personal right, S. 336; Zhang Xinbao, Legal protection of the right to privacy, S. 17. 
werden als Privatsphäre geschützt. Ob die Veröffentlichung, Verwendung der persönlichen Informationen eine Verletzung des Rechts auf Privatsphäre begründet, soll der Richter unter Berücksichtigung der Tatsache, wie die Informationen erhoben wurden, bzw. wie sie veröffentlicht wurden, des Umfangs, des Zieles und des Ergebnisses der Veröffentlichung und anderer Elemente entscheiden. ${ }^{305}$

Zweitens ist das Recht auf Privatsphäre kein absolut geschütztes Recht. Steht überwiegendes öffentliches Interesse oder Gruppeninteresse dem Recht auf Privatsphäre gegenüber, muss das Recht auf Privatsphäre zurücktreten. Nach chinesischem Recht ist eine Interessenabwägung bei der Beurteilung der Rechtsverletzung auf Privatsphäre immer notwendig. ${ }^{306}$ Die Arten der Privatsphäre begründen anders als in Deutschland keine Ausnahme.

$\mathrm{Zu}$ bemerken ist es noch, dass früher die Meinung herrschte, dass eine Rechtsverletzung auf Privatsphäre erst begründet ist, wenn die Veröffentlichung der Privatsphäre eine Ehrverletzung herbeiführt, also wenn die Veröffentlichung zu einer Senkung der sozialen Bewertung des Betroffenen führt oder wenn die Veröffentlichung dazu führt, dass der Betroffenen Schwierigkeiten bekommt, von der Gesellschaft weiter akzeptiert zu werden, und weiter dazu führt, dass der Betroffene psychisch unter Druck steht. ${ }^{307}$ Der Grund dieser Meinung liegt darin, dass der Schutz der Privatsphäre in China vor dem Inkrafttreten des Delikthaftungsgesetzes durch Ehrenschutz verwirklicht wurde. Auf diese Meinung soll jedoch verzichtet werden. Als Rechtsfolge der Verletzung des Rechts auf Privatsphäre reicht es, wenn die Privatsphäre ohne Einwilligung zu einem unbestimmten öffentlichen Kreis offengelegt wird. ${ }^{308}$

305 Peking Zweites Mitteleres Volksgericht, Urt. v. 23.12.2009 - (2009) er zhong min zhong zi di 5603 hao.

306 Shi Bifan, Zhejiang Social Sciences 2011, 34, 36.

307 Li Jun/Zhang Jun, People's Judicature (case) 2008, 24, 27.

308 Shi Bifan, Zhejiang Social Sciences 2011, 34, 36. 


\section{$\$ 6$ Der Schutz des Rechts der persönlichen Ehre}

\section{Der Schutz des Rechts der persönlichen Ehre in Deutschland}

Das Recht der persönlichen Ehre wird in Deutschland im Rahmen des allgemeinen Persönlichkeitsrechts (Art 2 Abs. 1 iVm Art 1 Abs. 1 GG) geschützt. ${ }^{309}$ Nach der herrschenden Meinung genießt das allgemeine Persönlichkeitsrecht als „sonstiges Recht“ den Schutz des $\$ 823$ Abs. 1 BGB. ${ }^{310}$ Deswegen besteht für Ehrverletzung die Möglichkeit, einen Anspruch nach $₫ 823$ Abs. 1 BGB in Verbindung mit Art. 1 und 2 Abs. 1 GG zu verlangen. ${ }^{311}$ Außerdem wird die Ehre auch durch $₫ 823$ Abs. 2 i.V.m. $\$ \$ 185$ ff StGB geschützt. ${ }^{312}$

Zivilrechtlich geschützt ist nur die äußere Ehre, nämlich der Ruf, das Ansehen einer Person in den Augen der anderen. ${ }^{313}$ Das subjektive Ehrgefühl einer Person ist hingegen nicht Schutzgegenstand des zivilrechtlichen Ehrenschutzes. ${ }^{314}$ Man kann nicht verlangen, in der Öffentlichkeit so dargestellt zu werden, wie man es selbst will. ${ }^{315}$

Dem Recht der persönlichen Ehre gegenüber steht am häufigsten die Meinungsfreiheit. Die Verfassungsgrundlage dafür steht in Art. 5 Abs. 2 GG. Nach der Rechtsprechung begründet die Meinungsäußerung die Verletzung des Rechts der persönlichen Ehre, wenn es sich um reine Schmähkritik handelt. ${ }^{316}$ Geht es um Tatsachenbehauptung, ist der Wahrheitsgehalt für die Rechtsverletzung

309 Kannowski in Staudinger, BGB Vorbemerkungen zu $₫ 1$, Rn. 28; Rixecker in Münchener Kommentar zum BGB, Anhang zu $\$ 12$, Rn. 78; Fink in Spindler/ Schuster, C. Verfassungsrecht, Rn. 60.

310 Siehe Teil $\$ 4$, I, 3.

311 Siehe Teil $\$$, I, 3.

312 Grabenwarter in Maunz/Dürig, GG Art. 5, Rn. 205.

313 Rixecker in Münchener Kommentar zum BGB, Anhang zu $\$ 12$, Rn. 79.

314 Grabenwarter in Maunz/Dürig, GG Art. 5, Rn. 209; Rixecker in Münchener Kommentar zum BGB, Anhang zu $\$ 12$, Rn. 79.

315 Vgl. BVerfG, GRUR 2011, 255, 257; BVerfGE 82, 236, 269; 101, 361, 380; 120, 180, 198.

316 BVerfGE 82, 272, 283 f.; 85, 1, 16; 93, 266, 294; Bamberger in Bamberger/Roth, BGB $\S 12$, Rn. 139. 
entscheidend. ${ }^{317}$ Die Offenlegung nur wahrer Tatsachen einer Person wird nicht im Rahmen des Ehrenschutzes diskutiert.

Die Beurteilung der Schmähkritik muss in jedem einzelnen Fall diskutiert werden. In Deutschland hat sich eine Reihe von Rechtsprechungen entwickelt, die auf unterschiedliche Situationen angewendet werden kann. ${ }^{318}$ Eigentlich besteht kaum ein Unterschied zwischen der traditionellen Ehrverletzung und der Ehrverletzung durch die Kommentare während der Menschenfleischsuche. Deswegen werden die Kriterien der Schmähkritik nicht besonders diskutiert.

\section{Der Schutz des Rechts der persönlichen Ehre in China}

Der Ehrschutz in China ist, wie oben bei dem Schutz der Privatsphäre schon erwähnt, in $\$ 101$ AGZR geregelt. Aufgrund dieses Paragraphen hat die natürliche Person das Recht auf Ehre; ihre Ehre darf nicht durch Beleidigung, Verleumdung oder andere Weise verletzt werden.

Für die Beurteilung einer Ehrverletzung muss nach $₫ 7$ „Antwort zu einigen

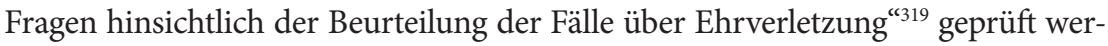
den, ob die Ehre des Betroffenen beschädigt ist, ob das Verhalten des Täters rechtswidrig ist, ob eine Kausalität zwischen dem rechtswidrigen Verhalten und dem Schaden besteht und, ob der Täter subjektiv schuld ist. Nach $₫ 8$ dieser Antwort sollen die Richter bei der Beurteilung der Ehrverletzung noch folgende Kriterien mitberücksichtigen: wenn die Äußerung über den Betroffenen hauptsächlich wahr ist und keinen beleidigten Inhalt enthält, soll keine Ehrverletzung vorliegen; wenn die Äußerung über den Betroffenen hauptsächlich wahr ist aber beleidigenden Inhalt enthält, und somit die Ehre tatsächlich beschädigt ist, soll eine Ehrverletzung vorliegen; wenn die Äußerung über den Betroffenen hauptsächlich unwahr ist, und damit die Ehre tatsächlich beschädigt ist, soll eine Ehrverletzung gegeben sein.

Die Tatsache, dass die Ehre tatsächlich beschädigt ist, ist eine entscheidende Voraussetzung einer Ehrverletzung. ${ }^{320}$ In der chinesischen Entscheidungspraxis ist es allgemein anerkannt, dass die Minderung der sozialen Bewertung der einzige Standard der tatsächlichen Beschädigung der Ehre ist. ${ }^{321} \mathrm{Ob}$ eine

317 Vgl. Bamberger in Bamberger/Roth, BGB $\$ 12$, Rn. 139; vgl. auch Schemmer in Epping/Hillgruber, GG Art. 5, Rn. 121.

318 Rixecker in Münchener Kommentar zum BGB, Anhang zu § 12, Rn. 79.

319 Vom chinesischen Obersten Volksgerichtshof.

320 Xiang Pin, The Rule of Law Forum 2011, 86, 87.

321 Zhongshan Mitteleres Volksgericht, Urt. v. 25.11.2005 - (2005) zhong zhong fa min yi zhong zi di 1003 hao; Kaifeng Mitteleres Volksgericht, Urt. v. 19.12.2011 - (2011) bian 
soziale Bewertung einer Person gemindert ist, soll nach der Meinung der Durchschnittsperson entschieden werden. ${ }^{322}$ Soziale Bewertung ist, wie schon der Name besagt, die Bewertung der Mitmenschen. Genau wie in Deutschland spielt das subjektive Gefühl des Betroffenen bei der Beurteilung der Ehrverletzung keine Rolle. ${ }^{323}$

Bei der Beurteilung der Ehrverletzung muss auch die Meinungsfreiheit berücksichtigt werden. Geht es um ein Werturteil, soll die intensive oder negative Kritik und die Beleidigung unterschieden werden. ${ }^{324}$ Nach der Rechtsprechung ist die intensive oder negative Kritik gegen den Betroffenen durch die Meinungsfreiheit geschützt, solange sie keinen beleidigenden Inhalt hat. ${ }^{325}$

Geht es um Tatsachenbehauptung, ist es nach $₫ 8$ „Antwort zu einigen Fragen hinsichtlich der Beurteilung der Fälle über Ehrverletzung "326 wichtig für eine Beurteilung, ob es sich bei der Äußerung um wahre Tatsachen handelt. Für die Beurteilung der Wahrheit der Tatsachenbehauptung wird nicht nur die tatsächliche Wahrheit geprüft, sondern auch, ob der Äußernde an die Wahrheit seiner Äußerung glaubt. ${ }^{327}$ Das ist für die Äußerung im Internet besonders wichtig, weil der Äußernde häufig nur aufgrund der im Internet zur Verfügung stehenden Information eine Schlussfolgerung erschließt, obwohl die Wahrheit solcher Information häufig nicht garantiert werden kann. Nach der Rechtsprechung begründet die unwahre Tatsachenbehauptung keine Ehrverletzung, solange der Äußernde seiner Fähigkeit entsprechend die Pflicht zur Prüfung der Wahrheit der Information erfüllt hat. ${ }^{328}$ Die Fähigkeit, um die Wahrheit der Information $\mathrm{zu}$ prüfen, unterscheidet sich zwischen unterschiedlichen Personen. Ein

min zhong zi di 1165 hao; Xi' an Mitteleres Volksgericht, Urt. v. 30.11.2012 - (2012) xi min er zhong zi di 02249 hao; Pukou Unteres Volksgericht, Urt. v. 12.12.2012 - (2012) pu min chu zi di 2125 hao; Jinhua Mitteleres Volksgericht, Urt. v. 26.03.2012 - (2012) zhe jin min zhong zi di 324 hao; Peking Oberes Volksgericht, Urt. v. 29.11.2007 (2007) gao min zhong zi di 1146 hao.

322 Zhongshan Mitteleres Volksgericht, Urt. v. 25.11.2005 - (2005) zhong zhong fa min yi zhong zi di 1003 hao.

323 Peking Oberes Volksgericht, Urt. v. 29.11.2007 - (2007) gao min zhong zi di 1146 hao.

324 Shanghai Mitteleres Volksgericht, Urt. v. 17.05.2012 - (2012) hu yi zhong min yi (min) zhong zi di 1086 hao; vgl. Xiang Pin, The Rule of Law Forum 2011, 86, 87.

325 Peking Oberes Volksgericht, Urt. v. 29.11.2007 - (2007) gao min zhong zi di 1146 hao.

326 Vom chinesischen Obersten Volksgerichtshof.

327 Han Gong, http://www.npc.gov.cn/huiyi/lfzt/qqzrfca/2008-12/21/content_1462861. htm (besucht am 04.04.2015).

328 Peking Oberes Volksgericht, Urt. v. 29.11.2007 - (2007) gao min zhong zi di 1146 hao. 
Journalist besitzt z.B. eine höhere Fähigkeit, die Wahrheit zu erkennen, als ein normaler Internetnutzer. ${ }^{329}$

Wenn eine Ehrverletzung begründet ist, hat der Rechtsinhaber aufgrund $\$ 120$ AGZR einen Anspruch auf Unterlassung des rechtswidrigen Verhaltens, Wiedergutmachung der Ehre, Beseitigung der durch die Rechtsverletzung entstehenden Einwirkung, offizielle Entschuldigung und Schadenersatz.

329 Zhongshan Mitteleres Volksgericht, Urt. v. 25.11.2005 - (2005) zhong zhong fa min yi zhong zi di 1003 hao. 


\section{$\$ 7$ Die Verhältnisse zwischen Schutz am eigenen Bild, Datenschutz, Schutz der Privatsphäre und Ehrenschutz}

\section{Das Verhältnis zwischen dem Schutz am eigenen Bild und Datenschutz}

\section{In Deutschland}

Wie oben schon diskutiert wurde, gilt BDSG nur auf die von dem ICP und dem ISP begangenen Rechtsverletzungen während der Menschenfleischsuche. Deswegen wird hier nur der Konflikt der Anwendbarkeit des KunstUrhG und BDSG bezüglich der Situation diskutiert, wenn das Bild der Zielperson ohne ihre Einwilligung auf der von dem ICP oder dem ISP betriebenen Webseite erscheint. Die Situation ist problematisch, weil das Bild der Zielperson als personenbezogenes Datum angesehen werden und Schutzgegenstand des BDSG sein könnte, während das Bild einer Person gleichzeitig der Schutzgegenstand des KunstUrhG ist.

Vom KunstUrhG geschützt ist das Bildnis mit erkennbarer Darstellung der Zielperson. ${ }^{330}$ Um Schutzgegenstand des BDSG zu werden, muss das Bildnis nach $₫ 3$ Abs. 1 BDSG Einzelangaben über persönliche oder sachliche Verhältnisse einer bestimmten oder bestimmbaren natürlichen Person enthalten. Aus einem Bildnis kann man z.B. Hautfarbe, persönliche Umstände, Hobbys oder mindestens die Gesichtszüge des Abgebildeten erkennen. ${ }^{331}$ Diese erkennbaren Informationen sind Einzelangaben über persönliche Verhältnisse. Aufgrund der Erkennbarkeit des Bildnisses kann eine natürliche Person bestimmt werden. ${ }^{332}$ Deswegen sind die vom KunstUrhG geschützten Bildnisse auch Schutzgegenstand des BDSG.

Über den Anwendungskonflikt zwischen BDSG und KunstUrhG wurde im Jahre 2008 ein Aufsatz geschrieben. ${ }^{333}$ Das Hauptargument zur Unterscheidung zwischen BDSG und KunstUrhG liegt darin, dass die datenschutzrechtliche Einwilligung und die kunsturheberrechtliche Einwilligung unterschiedliche

330 Fricke in Wandtke/Bullinger, KunstUrhG $\$ 22$, Rn. 5.

331 Schnabel, ZUM 2008, 657, 661.

332 Vgl. Schnabel, ZUM 2008, 657, 661.

333 Schnabel, ZUM 2008, 657. 
Formerfordernisse und Widerrufbarkeit haben. ${ }^{334}$ Dieser Unterschied spielt bei der Menschenfleischsuche jedoch keine Rolle, weil die Bildnisse der Zielperson ohne Einwilligung veröffentlicht sind. BDSG und KunstUrhG können gleichzeitig auf unsere Situation angewendet werden.

\section{In China}

Gemäß $₫ 1$ „Informationsschutz-Entscheidung“, der wichtigsten Regelung über Datenschutz in China, wird die digitale Information geschützt, mit der man eine Person identifizieren kann oder die der persönlichen Privatsphäre zuzuordnen ist. Wegen der Erkennbarkeit des Bildnisses ist der Abgebildete identifizierbar. Das bedeutet, die Information, die ein digitales Bildnis zeigt, ist die persönliche Information i.S.v. $\$ 1$ der „Informationsschutz-Entscheidung“.

Außerdem wird die vom Internetnutzer begangene Rechtsverletzung, wie oben diskutiert wurde, auch von dieser Entscheidung umfasst.

Das Recht am eigenen Bild gegen die willkürliche Veröffentlichung oder Verbreitung im Internet von dem Internetnutzer, dem ICP und dem ISP kann deshalb in China auch durch Datenschutz geschützt werden.

\section{Das Verhältnis zwischen Datenschutz und Schutz der Privatsphäre}

Datenschutz und Schutz der Privatsphäre haben unterschiedlichen Schutzumfang. Als Schutzgegenstand des Datenschutzes ist der Umfang der personenbezogenen Daten relativ objektiv. ${ }^{335}$ Alle personenbezogenen Daten, die Identifizierungsfunktion haben, gehören zu dem Schutzbereich. Dieser Standard unterscheidet nicht zwischen verschiedenen Personen. Im Gegenteil haben die zur Privatsphäre gehörenden persönlichen Informationen subjektive Merkmale. ${ }^{336}$ Der Umfang der Privatsphäre kommt auf jede einzelne Person an. Falls eine Person mit Absicht seine Privatsphäre der Öffentlichkeit offenlegt ${ }^{337}$ oder eine Person ihre persönlichen Informationen nicht als zur Privatsphäre gehörend behandelt ${ }^{338}$, gehören die bezüglichen Informationen dann nicht zur Privatsphäre. ${ }^{339}$

334 Schnabel, ZUM 2008, 657, 659ff.

335 Shi Jiayou, Journal of the University of Suzhou 2012, 85, 87.

336 Shi Jiayou, Journal of the University of Suzhou 2012, 85, 87.

337 Shi Jiayou, Journal of the University of Suzhou 2012, 85, 87.

338 Shi Jiayou, Journal of the University of Suzhou 2012, 85, 87.

339 Vgl. Huang Ping, Journal of Shanghai Institute of Political Science \& Law 2009, $16,17$. 
Nicht alle zur Privatsphäre gehörenden Rechtsgüter stehen unter dem Schutzbereich des persönlichen Datenschutzes. Das Recht in Ruhe gelassen zu wer$\operatorname{den}^{340}$ z.B. kann nicht durch Datenverarbeitungsanlagen digitalisiert werden. Solche Rechtsgüter können nur durch den Schutz der Privatsphäre geschützt werden. ${ }^{341}$

Manche vom Datenschutzgesetz geschützten Informationen gehören nicht zur Privatsphäre. Falsche Informationen z.B. sind auch Schutzobjekt des Rechts auf informationelle Selbstbestimmung. Der Betroffene kann eine Korrektur auf die richtigen Informationen verlangen. Jedoch gehören die falschen Informationen überhaupt nicht zur Privatsphäre. ${ }^{342}$ Vor der Veröffentlichung falscher persönlicher Informationen wird der Betroffene durch Ehrenschutz geschützt.

Trotz Unterschiedes haben das Recht auf informationelle Selbstbestimmung und das Recht auf Privatsphäre nach meiner Meinung einen gemeinsamen Schutzbereich, nämlich die zur Privatsphäre gehörenden Informationen, die digitalisierbar sind und Identifikationsfunktion haben. ${ }^{343}$

\section{In Deutschland}

Spezifisch auf den Fall der Menschenfleischsuche haben das Recht auf informationelle Selbstbestimmung und das Recht auf Privatsphäre gemäß deutschem Recht unterschiedliche Verteidigungsgegner. Zum Schutz des Rechts auf informationelle Selbstbestimmung wird das BDSG hauptsächlich gegen ISP angewendet, während der Verteidigungsgegner des Rechts auf Privatsphäre häufig der Internetnutzer ist.

Für den oben genannten gemeinsamen Schutzbereich soll durch die zwei Schutzwege das gleiche Ergebnis erreicht werden. Das heißt z.B., wenn die Meinungsfreiheit durch ein schutzwürdiges öffentliches Informationsinteresse bei der Interesseabwägung zwischen Meinungsfreiheit und Schutz der Privatsphäre begründet ist, muss $₫ 28$ Abs. 1 Satz 1 BDSG bei der Interesseabwägung zwischen Meinungsfreiheit und Schutz des Rechts auf informationelle Selbstbestimmung ebenfalls zugunsten der Meinungsfreiheit ausgelegt werden. ${ }^{344}$ Das gleiche gilt

340 Bamberger in Bamberger/Roth, BGB $\$ 12$, Rn. 140.

341 Vgl. Jing Huibao, Journal of Law Application 2011, 90, 92; und Mei, Shaozu, Academic Journal of Suzhou University (Philosophy and Social Science) 2005, 25, 27; siehe auch Lian Xiao, Journal of Heilongjiang Administrative Cadre Institute of Politics and Law 2010, 58.

342 Vgl. Shi Jiayou, Journal of the University of Suzhou 2012, 85, 87.

343 Vgl. Yao Yuerong, Political Science and Law 2012, No. 4, 72, 63.

344 Härting, CR 2009, 21, 28. 
auch umgekehrt. Der Schutz des Rechts auf informationelle Selbstbestimmung und der Schutz der Privatsphäre dienen beide grundsätzlich dem Schutz der Persönlichkeitsrechte. Ein wesentlicher Konflikt zwischen den beiden Schutzwegen soll nicht bestehen. ${ }^{345}$

\section{In China}

Nach $₫ 1$ „Informationsschutz-Entscheidung“ sind alle digitalen Daten, die Privatsphäre betreffen, von der Entscheidung geschützt. Die zur Privatsphäre gehörenden Informationen, die in digitaler Form sind, sind in China gleichzeitig Gegenstand des Datenschutzes und Schutzes der Privatsphäre.

\section{Schutz der allgemeinen Persönlichkeitsrechte}

Außer den oben genannten zwei Verhältnissen bestehen zwischen dem Schutz am eigenen Bild, dem Datenschutz, dem Schutz der Privatsphäre und dem Ehrenschutz noch zahlreiche Verbindungen und Unterschiede. Besonders für den Fall der Menschenfleischsuche passiert es häufig, dass der Verletzte durch mehrere Wege geschützt werden kann.

\section{In Deutschland}

In Deutschland gehören diese vier Schutzwege zum Schutz der Persönlichkeitsrechte. Um eine Verletzung des Persönlichkeitsrechts während der Menschenfleischsuche zu begründen, reicht allein die Verwirklichung des Eingriffstatbestandes nicht. ${ }^{346}$ Eine Abwägung zwischen dem schutzwürdigen Interesse des Betroffenen und der Meinungsfreiheit oder anderen Interessen des verdächtigen Verletzers und dem Auskunftsrecht der Öffentlichkeit ist in jedem einzelnen Fall notwendig. ${ }^{347}$

Eine Unterscheidung zwischen dem allgemeinen und besonderen Persönlichkeitsrecht ist hier nicht erforderlich, weil eine Interessenabwägung auch für das Recht am eigenen Bild von $\$ 23$ KunstUrhG ausdrücklich gefordert wird.

345 Vgl. Schnabel, ZUM 2008, 657, 658; und Bamberger in Bamberger/Roth, BGB $₫ 12$, Rn. 140.

346 Rixecker in Münchener Kommentar zum BGB, Anhang zu $₫ 12$, Rn. 132 ff; Nink in Spindler/Schuster, BGB $\$ 823$, Rn. 5.

347 Nink in Spindler/Schuster, BGB $\$ 823$, Rn. 5; BVerfG, NJW 1987, 2669, 2670; BGHZ 13, 338; BGH, NJW 1981, 1366. 
Außerdem ist allgemeines Persönlichkeitsrecht ein Auffangrecht. ${ }^{348}$ Zum Schutz des besonderen Persönlichkeitsrechts ist ein Rückgriff auf das Allgemeine Persönlichkeitsrecht stets möglich. ${ }^{349}$

Das allgemeine Persönlichkeitsrecht als Auffangrecht der oben genannten vier konkreten Rechte hat nach meiner Meinung weiterhin zur Folge, dass alle Interessenabwägungen, egal durch welchen in Konflikt geratenen Schutzweg, das gleiche Ergebnis erzielen sollen. ${ }^{350}$

Übrigens darf durch die Interessenabwägung keine gesonderte Beschränkung der gegenüberstehenden Rechtsgüter, wie z.B. Meinungsfreiheit, entwickelt werden, die über die Schranken durch traditionelles allgemeines Persönlichkeitsrecht hinausgehen. ${ }^{351}$

\section{In China}

In China gibt es im Gesetz bisher noch kein allgemeines Persönlichkeitsrecht. Es gibt aber bereits Diskussionen darüber ${ }^{352}$, ob China beim Entwerfen des neuen Bürgerlichen Gesetzbuchs das allgemeine Persönlichkeitsrecht als Auffangrecht ausdrücklich mit aufnehmen soll.

Zum Schutz des eigenen Bildes, der Privatsphäre, der persönlichen Daten und der persönlichen Ehre gibt es in China deutliche Regelungen. Diese Regelungen sind jedoch nur über die Eingriffstatbestände der Rechtsverletzungen dieser vier Rechtsgüter. Ein Bedarf auf Interessenabwägung im einzelnen Fall wird in diesen Regelungen nicht erwähnt. So wie der Schutz des Rechts am eigenen Bild genießen die vier Rechtsgüter in China laut des Gesetztexts häufig nur „Ein-Stufen-Schutz“.

Um die Rechte am besten zu schützen wird der Verletzte beim Konfliktfall in der Praxis Ansprüche wegen aller möglichen Rechtsverletzungen verlangen. ${ }^{353}$ In der Praxis ist es die Aufgabe der Richter, aufgrund ihrer Fachkenntnisse durch die Auslegung der Grenzen der vier Rechtsgüter eine Interessenabwägung tatsächlich durchzuführen.

348 Nink in Spindler/Schuster, BGB $\$ 823$, Rn. 6.

349 BGH, NJW 1996, 1128, 1129; NJW 1996, 985, 986; Nink in Spindler/Schuster, BGB $\$ 823$, Rn. 6 .

350 Vgl. mit dem Konfliktfall vom Datenschutz und Schutz der Privatsphäre unter $\$ 7$ II 1.

351 Härting, CR 2009, 21, 28.

352 Vgl. Wang Liming, Chinese Journal of Law 2003, 32.

353 Zhongshan Mitteleres Volksgericht, Urt. v. 25.11.2005 - (2005) zhong zhong fa min yi zhong zi di 1003 hao. 



\section{Kapitel. Die Identifizierung der Rechtsverletzungen während Menschenfleischsuche}





\section{$\$ 8$ Die von den Internetnutzern begangenen Rechtsverletzungen}

\section{Die von den Informationsberichtern begangenen Rechtsverletzungen}

Der Informationsberichter ist derjenige, der die persönlichen Informationen der Zielperson ohne dessen Einwilligung zum ersten Mal im Internet veröffentlicht hat.

\section{Nach deutschem Recht}

Das Verhalten des Informationsberichters könnte in Deutschland eine Verletzung des Rechts auf Privatsphäre begründen. Wären die veröffentlichten Informationen in der Form von Bildnissen der Zielperson, könnte auch eine Verletzung des Rechts am eigenen Bild begründet werden. Eine Rechtsverletzung der informationellen Selbstbestimmung wird damit nicht begründet. ${ }^{354}$

\section{a) Verletzung des Rechts auf Privatsphäre}

Zum Schutz des Rechts auf Privatsphäre ist die Sphärentheorie anwendbar. Zuerst soll untersucht werden, zu welcher Sphäre die vom Informationsberichter veröffentlichte persönliche Information gehört. Die Veröffentlichung der Information aus der Intimsphäre ohne ausdrückliche Einwilligung ist ständig verboten. ${ }^{355}$ Die Veröffentlichung der Information aus der Privatsphäre ist grundsätzlich unzulässig ${ }^{356}$; Ausnahmen sind allerdings möglich ${ }^{357}$. Die Veröffentlichung der Information aus der Sozialsphäre ist dagegen grundsätzlich erlaubt, solange der Betroffene daran kein überwiegendes Interesse hat. ${ }^{358}$ Zum Schutz der Informationen aus der Privatsphäre und der Sozialsphäre ist eine Interessenabwägung im einzelnen Fall notwendig, während eine Interessenabwägung im Fall vom

354 Siehe oben unter $\$ 4$ I 2 b).

355 Ballhausen/Roggenkamp, K\&R 2008, 403, 405.

356 Ballhausen/Roggenkamp, K\&R 2008, 403, 405.

357 EGMR, NJW 1999, 1315, 1316; BVerfGE 35, 202, 221; BVerfG, NJW 2000, 2189; BVerfG, AfP 2001, 212, 214f.; Nink in Spindler/Schuster, BGB $₫ 823$, Rn. 24.

358 BGH, AfP 1995, 404, 407; Nink in Spindler/Schuster, BGB \$ 823, Rn. 25. 
Schutz der Intimsphäre ausgeschlossen ist. ${ }^{359}$ Diese Unterscheidung macht aber nur Sinn, wenn man die drei Sphären, oder mindestens die Intimsphäre von den anderen zwei Sphären deutlich unterscheiden kann, was in der Praxis jedoch nicht immer der Fall ist. ${ }^{360}$

Bei einem Tagebuch, das normalerweise in der Intimsphäre einsortiert wird, ${ }^{361}$ gab es unterschiedliche höchstrichterliche Entscheidungen darüber, ob das Tagebuch zur Aufklärung einer schweren Straftat des Tagebuchführers verwendet werden darf. ${ }^{362}$ Der BGH hat in einer Entscheidung wegen Mordes vom 9.7.1987 die Verwertungsmöglichkeit des Tagesbuchs wegen eines überwiegenden öffentlichen Interesses bejaht, ${ }^{363}$ während das BVerfG die Auffassung vertreten hat, dass vor einer Interessenabwägung entschieden werden muss, ob der zu verwertende Inhalt des Tagebuchs zum „Kernbereich privater Lebensgestaltung“ gehört. $^{364}$

Das gleiche Problem ist auch beim Sexualleben vorhanden. Nach der aktuellsten Entscheidung des BGH fallen die sexuellen Vorlieben auch nicht per se unter die absolut geschützte Intimsphäre. ${ }^{365}$

In der Literatur vertritt die Meinung, dass bei den zur Intimsphäre zählenden Fällen ebenso eine Interessenabwägung durchgeführt werden soll. ${ }^{366} \mathrm{Zu}$ dieser Meinung stimme ich zu. Die Überlegung, zu welcher Sphäre die persönliche Information gehört, ist meiner Meinung nach schon eine Art von Interessenabwägung. Die Frage, ob ein Tagebuch oder die sexuellen Vorlieben zum Kernbereich privater Lebensgestaltung, also zur Intimsphäre, gehört, muss in jedem einzelnen Fall unter Berücksichtigung der konkreten Situation für die betroffene Person entschieden werden. Eine Interesseabwägung ist schon mit dieser Frage angefangen. Als Umstritten, zu welcher Sphäre die bezügliche Information gehört, ist es sinnvoller, dass man für jeden Fall von Anfang an eine

359 BVerfG, NJW 1990, 563, 565; BGH, NJW 1985, 1617, 1618; Nink in Spindler/ Schuster, BGB $\$ 823$, Rn. 23.

360 Vgl. Hager in Staudinger, Eckpfeiler des Zivilrechts, T. Das Recht der unerlaubten Handlungen, Rn. 344 und Nink in Spindler/Schuster, BGB \$ 823, Rn. 23; vgl. auch NStZ 1990, 397; und Ellbogen, NStZ 2001, 460, 462; sowie Amelung, NJW 1990, 1753, 1755.

361 Nink in Spindler/Schuster, BGB \$823, Rn. 23.

362 BGHSt 19, 325, 333 = NJW 1964, 1139; BGHSt 34, 397, 401 = NJW 1988, 1037; vgl. LG Saarbrücken, NStZ 1988, 424; Amelung, NJW 1990, 1753, 1754.

BGHSt 34, 397ff. = NJW 1988, 1037ff.

364 BVerfGE 80, 373, 375 = NJW 1990, 563; BVerfGE 6, 32, 41, 389, 433; 34, 238, 245.

365 BGH, NJW 2013, 1681 = AfP 2013, 250; Sajuntz, NJW 2014, 25, 26.

366 Hager in Staudinger, Eckpfeiler des Zivilrechts, T. Das Recht der unerlaubten Handlungen, Rn. 344. 
Interessenabwägung durchführt. Eine Unterscheidung der Sphären ist meiner Meinung nach unnötig. Auch für den Bereich der Intimsphäre ist eine Interessenabwägung notwendig.

Zusammengefasst ist in jedem einzelnen Fall zu prüfen, ob die vom Informationsberichter offengelegte Information über die Zielperson zur Verletzung des Rechts auf Privatsphäre führt. Dafür ist eine Interessenabwägung zwischen dem Persönlichkeitsrecht und anderen Rechten und Interessen für alle Fälle nötig. Umfangreiche Rechtsprechung und Literatur sind entwickelt worden, um die Interessenabwägung in unterschiedlichen Situationen zu konkretisieren. ${ }^{367}$ Dies wird unten in $₫ 8 \mathrm{~V}$ diskutiert.

\section{b) Verletzung des Rechts am eigenen Bild}

Gemäß $\$ 23$ Abs. 1 KunstUrhG dürfen ohne Einwilligung nur die Bildnisse verbreitet und zur Schau gestellt werden, die aus dem Bereich der Zeitgeschichte sind; oder auf denen die Personen nur als Beiwerk neben einer Landschaft oder sonstigen Örtlichkeit erscheinen; oder die von Versammlungen, Aufzügen und ähnlichen Vorgängen sind, an denen die dargestellten Personen teilgenommen haben; oder die nicht auf Bestellung angefertigt sind, sofern die Verbreitung oder Schaustellung einem höheren Interesse der Kunst dient. Die vom Informationsberichter veröffentlichten Bildnisse fokussieren sich immer auf die Zielperson. Deswegen ist nur die Kategorie „die Bildnisse aus dem Bereiche der Zeitgeschichte" in Betracht zu ziehen.

Unter dem Begriff der Zeitgeschichte ist das Informationsinteresse der Öffentlichkeit zu verstehen. ${ }^{368}$ In Verbindung mit $\$ 23$ Abs. 2 KunstUrhG ist dann eine Abwägung zwischen dem Informationsinteresse der Öffentlichkeit und den schutzwürdigen Interessen der Zielperson notwendig, um die Rechtsverletzung des Informationsberichters zu begründen.

Das Informationsinteresse der Öffentlichkeit an der Zielperson hängt von dem Ziel (bzw. die Motivation ${ }^{369}$ ) jeder Menschenfleischsuche ab, das durch die Veröffentlichung der Bildnisse verfolgt wird. Das heißt, solange das Informationsinteresse der Öffentlichkeit an der Zielperson ihr berechtigtes Interesse überwiegt, darf der Informationsberichter ohne ihre Einwilligung ihr Bildnis zum Ziel der Menschenfleischsuche veröffentlichen.

367 Kannowski in Staudinger, BGB Vorbemerkungen zu $\$ 1$, Rn. 25.

368 BVerfG, GRUR 2000, 446, 452; AfP 2001, 212, 215; Fricke in Wandtke/Bullinger, KunstUrhG $\$ 23$, Rn. 3.

369 Siehe oben unter $₫ 1$ III 1. 
Die Abwägung zwischen den schutzwürdigen Interessen der Zielperson und das Informationsinteresse der Öffentlichkeit während der Menschenfleischsuche wird im Teil $\$ 8 \mathrm{~V}$ diskutiert.

\section{Nach chinesischem Recht}

Durch das Verhalten des Informationsberichters kann eine Rechtsverletzung der Privatsphäre gemäß $\$ 2$ Delikthaftungsgesetz begründet werden, wenn die veröffentlichte Information zur Privatsphäre gehört. Die Veröffentlichung der Information, die nicht zur Privatsphäre gehört, begründet gemäß $₫ 1$ und $₫ 11$ "Informationsschutz-Entscheidung" auch eine Verletzung des zivilrechtlichen Interesses, solange die Information eine Identifikationsfunktion hat. Weil das Recht auf informationelle Selbstbestimmung im chinesischen Rechtssystem noch nicht besteht, kann es als zivilrechtliches Interesse wiederum nur als das Recht auf Privatsphäre geschützt werden, weil ein allgemeines Persönlichkeitsrecht in China ebenfalls fehlt.

Wenn die persönlichen Informationen in der Form von Bildnissen veröffentlicht sind, kann auch eine Verletzung des Rechts am eigenen Bild begründet werden.

\section{a) Verletzung des Rechts auf Privatsphäre}

Die Veröffentlichung der persönlichen Informationen der Zielperson ohne ihre Einwilligung durch den Informationsberichter während der Menschenfleischsuche könnte nach chinesischem Recht eine Verletzung des Rechts auf Privatsphäre begründen. Eine Ausnahme könnte jedoch bestehen, wenn das Recht auf Privatsphäre als Rahmenrecht in China behandelt würde. Das ist zwar nicht im Gesetz deutlich geschrieben, aber durchaus möglich. Die Beschränkung des Rechts auf Privatsphäre findet erstens ihre Grundlage im Art. 51 Verfassungsgesetz der Volksrepublik China: die Ausübung der Freiheiten und Rechte eines Bürgers darf das Interesse des Staats, der Gesellschaft, des Kollektivs und die rechtmäßigen Freiheiten und Rechte der anderen Bürger nicht beschädigen. Weiterhin sollen die zivilrechtlichen Aktivitäten gemäß $\$ 7$ AGZR die öffentliche Moral respektieren, und das öffentliche Interesse nicht beschädigen. Nach diesen Vorschriften stehen das Recht und die Freiheit des Informationsberichters und das öffentliche Interesse im Fall von Menschenfleischsuche dem Recht auf Privatsphäre eines Bürgers gegenüber. Auch nach $₫ 12$ der aktuellsten „Regelung vom Obersten Volksgericht über einigen Fragen hinsichtlich der anzuwendenden Gesetzen bei der Beurteilung von zivilrechtlichen Streitigkeiten wegen Verletzung der persönlichen Rechte und Interesse durch Internet“ dürfen die persönliche Information 
und die persönliche Privatsphäre im Internet offengelegt werden, wenn die Offenlegung zum öffentlichen Interesse dient und zweckmäßig ist.

Um die Rechtsverletzung des Informationsberichters zu begründen, ist eine Interessenabwägung nach chinesischem Recht durchaus möglich. Aber in der Entscheidungspraxis wird für diesen Fall keine Interessenabwägung durchgeführt. Die Gerichte in China bevorzugen es, durch die Subsumtion nach jedem Bestandteil des Eingriffs - der Tat, der Schuld, dem Ergebnis und der Kausalität um eine Rechtsverletzung zu prüfen. ${ }^{370}$ Bei der Prüfung der Eingriffstatbestände sind zwei Elemente für unseren Fall problematisch. Ein Element ist die Schuld des Informationsberichters. Das andere ist die Beurteilung, ob die bezügliche Information zur Privatsphäre gehört.

Gemäß $₫ 6$ OVG-AGZR-Ansichten und $₫ 8$,Informationsschutz-Entscheidung" ist die Veröffentlichung und Verbreitung der Privatsphäre ausdrücklich verboten. Die Schuld des Informationsberichters wäre gegeben, wenn er wüsste oder wissen sollte, dass die von ihm veröffentlichte Information zur Privatsphäre gehört.

Bei der Beurteilung einer Rechtsverletzung der Privatsphäre vom Informationsberichter kommt es zum Schluss nur noch auf die Frage an, ob die vom Informationsberichter veröffentlichten Informationen zur Privatsphäre gehören. Diese Frage wurde in dieser Arbeit unter $\$ 5$ II 2 bereits diskutiert. Über den Umfang der Privatsphäre gibt es keine feste Regelung. Dadurch ist ein Spielraum für die Beurteilung des Umfangs der Privatsphäre geschaffen worden. Diese Beurteilung hat die Funktion der Interessenabwägung übernommen. In der Entscheidungspraxis nutzen die Gerichte diese Chance, den Umfang der Privatsphäre enger oder breiter auszulegen, um tatsächlich eine Interessenabwägung durchzuführen. ${ }^{371}$

$\mathrm{Zu}$ ergänzen ist, dass die persönliche Information im Internet, die zwar nicht der Privatsphäre zuzuordnen ist, aber Identifikationsfunktion hat, gemäß $₫ 1$ und $₫ 8$ „Informationsschutz- Entscheidung“ ebenso als Privatsphäre geschützt werden könnte. Gemäß $\$ 1$ „Informationsschutz- Entscheidung“ werden nicht nur die Privatsphäre betroffene Information sondern auch die persönliche Information, die Identifikationsfunktion hat, vom Staat geschützt. Im Fall von willkürlicher Offenlegung der Information von den beiden Sorten hat die betroffene Person gemäß $\$ 8$ „Informationsschutz- Entscheidung“ den gleichen

370 Xiong Jingbo, Ecupl Journal 2011, 28, 33f; vgl. Zhongshan Mitteleres Volksgericht, Urt. v. 25.11.2005 - (2005) zhong zhong fa min yi zhong zi di 1003 hao.

371 Vgl. Zhongshan Mitteleres Volksgericht, Urt. v. 25.11.2005 - (2005) zhong zhong fa min yi zhong zi di 1003 hao und Peking Zweites Mitteleres Volksgericht, Urt. v. 23.12.2009 - (2009) er zhong min zhong zi di 5603 hao. 
Anspruch, um ihr Interesse zu schützen. Nach dieser Auslegung wird die persönliche Information im Internet geschützt, solange sie Identifikationsfunktion hat, egal ob sie als Privatsphäre einsortiert werden kann. Wegen der unklaren Grenze der Privatsphäre kann die persönliche Information im Internet hierdurch besser geschützt werden, weil die Begründung, dass die persönliche Information Identifikationsfunktion hat, viel leichter ist, als zu begründen, dass die persönliche Information zur Privatsphäre gehört. Dieser Gesichtspunkt ist der Literatur und der Entscheidungspraxis jedoch noch nicht aufgefallen.

\section{b) Verletzung des Rechts am eigenen Bild}

Anders als das Recht auf Privatsphäre ist eine Interessenabwägung bei der Beurteilung der Verletzung des Rechts am eigenen Bild in der Praxis anerkannt. ${ }^{372}$ Gemäß $\$ 24$ „Handbuch über Eröffnung des Gerichtsverfahrens“ kann ein Bild ohne die Einwilligung des Abgebildeten verwendet werden, wenn die Verwendung für Zwecke des öffentlichen Interesses, des eigenen Interesses des Bürgers, der Rechtspflege, der Berichterstattung, der wissenschaftliche Forschung oder der Bildung dient. Für den Fall der Menschenfleischsuche, die in dieser Arbeit diskutiert wird, kommt nur die Abwägung zwischen dem öffentlichen Interesse und dem Interesse des Abgebildeten in Frage. Auch diese Interessenabwägung wird unten im Teil $₫ 8 \mathrm{~V}$ diskutiert.

\section{Die von den Informationssammlern begangenen Rechtsverletzungen}

Der Informationssammler ist derjenige vom Veranlasser motiviert, der unter Benutzung einer normalen Suchmaschine (wie z.B. Google) und anderer Internetseiten (wie z.B. soziales Netzwerk wie Facebook) die im Internet schon veröffentlichten Informationen sammelt, sortiert, analysiert, zusammenfasst und die zusammengefassten Informationen in dem Forum, wo die Menschenfleischsuche stattfindet, einträgt. ${ }^{373}$

\section{Die Verwendung der ohne Zustimmung veröffentlichten Informationen vom Informationssammler}

Die schon veröffentlichten Informationen sind nicht unbedingt mit der Zustimmung des Rechtsinhabers offengelegt. Hier ist es zuerst zu prüfen, ob die ohne

372 Siehe oben unter $\$ 3$ II 2.

373 Zhou Xiaochen, Netinfo Security 2008, 13, 14. 
Zustimmung veröffentlichten persönlichen Informationen von den Internetnutzern frei verwendet werden dürfen.

\section{a) In Deutschland}

Es gibt im Internet eine unglaublich große Menge von Informationen. Es ist für einen normalen Internetnutzer schlecht möglich zu prüfen, ob im Internet stehende Informationen mit der Einwilligung oder Genehmigung des Rechtsinhabers veröffentlicht sind. ${ }^{374}$ Deswegen soll die Situation hier nach dem Empfängerhorizont behandelt werden, um eine Zensur der freien Meinungsäußerung zu vermeiden. Das heißt, die schon veröffentlichte persönliche Information kann normalerweise frei verwendet werden, wenn eine normale Person nach allgemeiner sorgfältiger Prüfung nicht erkennen kann, dass die Information ohne jegliche Zustimmung veröffentlicht wurde. Mit anderen Worten, der Informationssammler muss bezüglich der Verwendung solcher Informationen gutgläubig sein.

\section{b) In China}

Das Gleiche Prinzip soll auch für China gelten. ${ }^{375}$ Ein Beispiel hat das Obere Volksgericht in Jiangxi, eine Provinz in China, in seiner „Leitlinie einiger Fragen über die Gesetzesanwendung für die Beurteilung der Fälle über Rechtsverletzung im Internet (Probe-Version)“ genannt. Gemäß $\$ 22$ dieser Leitlinie soll der Internetnutzer für den wegen seines Verhaltens entstehenden weiteren Schaden als Gesamtschuldner haften, wenn er weiß, dass die Inhalte rechtswidrig sind und sie trotzdem mit gewerblichen Zweck weiterleitet und verbreitet; oder die rechtswidrigen Inhalte für andere Interessen organisiert weiterleitet oder verbreitet, und als Ergebnis zu einer Verbreitung der rechtswidrigen Inhalte führt. $\mathrm{Zu}$ den rechtswidrigen Inhalten gehören die persönlichen Informationen, die ohne Zustimmung des Rechtsinhabers veröffentlicht wurden.

Die ohne und mit Zustimmung veröffentlichen Informationen werden für die weitere Verwendung durch die Internetnutzer gleich behandelt, solange es nicht offensichtlich ist, dass die Information ohne Zustimmung des Betroffenen veröffentlicht wird. Ob diese Informationen zum beliebigen Zweck von den Internetnutzern verwendet werden dürfen, ist die weitere Frage. Besonders Problematisch wäre es, wenn die Internetnutzer die Informationen zum rechtswidrigen Zweck verwenden.

374 Seidel/Nink, CR 2009, 666, 670.

375 Topic Research Group of No.3 Criminal Division, People's Judicature Appliction 2012, 17, 20. 


\section{Die Verwendung der frei zugänglichen Informationen zum beliebigen Zweck}

\section{a) In Deutschland}

Die Frage ist, ob die zum anderen Zweck im Internet eingetragene Information weiterhin zum Zweck der Menschenfleischsuche verwendet werden darf. In Deutschland dürfen die persönliche Daten nach dem im BDSG geregelten Zweckbindungsgrundsatz grundsätzlich nur zu dem primären Zweck verarbeitet und genutzt werden, zu dem sie erhoben oder erfasst worden sind. ${ }^{376}$ Jedoch gilt BDSG nicht auf der vom Informationssammler begangenen Rechtsverletzung. Dies wurde bereits unter $\$ 4 \mathrm{I} 2 \mathrm{~b}$ ) diskutiert. Für die vom Informationssammler begangene Rechtsverletzung sind die allgemeinen Vorschriften anwendbar.

Aus dem Grundsatz der Privatautonomie gemäß Art. 2 des deutschen Grundgesetzes ist das Prinzip anzuerkennen, dass alles grundsätzlich erlaubt ist, was nicht in verfassungsmäßiger Weise verboten ist. ${ }^{377}$ Jedoch hören die Rechte von einem da auf, wo die Rechte anderer anfangen. In unserem Fall hören die Rechte des Informationssammlers auf, wenn er die persönliche Information anderer Person zum rechtswidrigen Zweck weiter verwendet. Solche Verwendung wird wegen der Rechtwidrigkeit eine Rechtsverletzung im Sinne von $\$ 823$ Abs. 1 BGB begründen. ${ }^{378}$

$\mathrm{Ob}$ die Informationssammler während Menschenfleischsuche die frei zugängliche Information zum rechtswidrigen Zweck verwendet, muss in jedem einzelnen Fall geprüft werden. Offensichtlich rechtswidrig ist zum Beispiel der Zweck, um Rache auszuüben. ${ }^{379}$

\section{b) In China}

Allein die Verbreitung der schon veröffentlichten Informationen ohne eine Änderung begründet in China normalerweise keine Rechtsverletzung des Persönlichkeitsrechts. ${ }^{380}$ Jedoch ist es fraglich, ob die mit einem bestimmen Zweck veröffentlichten Informationen für weiteren beliebigen Zwecke verwendet werden dürfen.

376 Dammann in Simitis, BDSG, \$ 14 Rn. 37 ff.

377 Schmidt in Dieterich/Müller-Glöge, GG Art. 2, Rn. 1.

378 Teichmann in Jauernig/Stürner/Berger, BGB $\$ 823$, Rn. 49.

379 Siehe oben unter $\$ 1$ III $1 \mathrm{f}$ ).

380 Topic Research Group of No.3 Criminal Division, People's Judicature Appliction 2012, 17, 20. 
$\mathrm{Zu}$ dieser Frage gibt es in der chinesischen Literatur unterschiedliche Meinungen. ${ }^{381}$ Nach der herrschenden Meinung bedeutet die tatsächliche Offenlegung der persönlichen Informationen einen Verzicht auf den Privatsphärenschutz auf diese Informationen; ${ }^{382}$ deren Verwendung ist ständig erlaubt, solange sie keinem rechtswidrigen Zweck dienen. ${ }^{383}$

Die herrschende Meinung wurde von der neuesten „Regelung vom Obersten Volksgericht über einigen Fragen hinsichtlich der anzuwendenden Gesetzen bei der Beurteilung von zivilrechtlichen Streitigkeiten wegen Verletzung der persönlichen Rechte und Interesse durch Internet ${ }^{\text {“384 }}$ bejaht. Aufgrund $\$ 12$ Abs. 1 Nr. 5 und 6 dürfen Internetnutzer die persönliche Information veröffentlichen bzw. weiterleiten, wenn die Information von der betroffenen Person selbst oder durch andere Weise legal veröffentlicht wurde, oder wenn die Information durch rechtmäßigen Weg erhoben wurde. Dieser Absatz ermöglicht dem Informationssammler, die persönliche Information der Zielperson während Menschenfleischsuche zu sammeln und weiterzuleiten. Aber wie in Deutschland hat diese Regelung auch Beschränkungen für die Verwendung persönlicher Information der Zielperson festgelegt. Aufgrund $\$ 12$ Abs. 2 der Regelung darf die oben genannte frei zugängliche persönliche Information nicht auf die Weise verwendet werden, die öffentliches Interesse oder öffentliche Moral widrig ist; solche Information darf weiterhin auch nicht verwendet werden, wenn die Zielperson daran überwiegend zu schützendes Interesse hat.

\section{Zwischenergebnis}

Es soll nach Empfängerhorizont geprüft, ob der Informationssammler weiß, dass die von ihm verwendete veröffentlichte Information bei der Veröffentlichung rechtswidrig war. Wenn er nach Ausübung der Sorgfaltspflicht des normalen Bürgers nicht erkennen kann, dass die Information ohne Zustimmung der Zielperson veröffentlicht war, darf er sie frei verwenden, solange die Verwendung keinem rechtswidrigen Zweck dient.

381 Topic Research Group of No.3 Criminal Division, People's Judicature Appliction 2012, 17, 20; vgl. Dai Yingjie, Journal of Zhejiang Institute of Media and Communications 2011, 37, 39.

382 Datenschutz in China ist durch Schutz der Privatsphäre verwirklicht. Siehe oben unter $\$ 4$ II.

383 Dai Yingiie, Journal of Zhejiang Institute of Media and Communications 2011, 37, 39.

384 Erlassen am 23.6.2014, gültig ab 10.10.2014. 
Die Verwendung der frei zugänglichen Information durch Informationssammler begründet nach der deutlichen Regelung des Obersten Volksgerichts in China keine Rechtsverletzung, solange die Verwendung nicht öffentliches Interesse oder öffentliche Moral widrig ist, und die betroffene Person daran kein überwiegend zu schützendes Interesse hat. Die ähnliche Regelung findet man auch im deutschen Recht. Gemäß Art. 2 Abs. 1 des deutschen Grundgesetzes endet die Handlungsfreiheit einer Person, wenn sie die Rechte anderer verletzt oder gegen die verfassungsmäßige Ordnung oder das Sittengesetz verstößt.

Zur Beurteilung der vom Informationssammler begangenen Rechtsverletzung gibt es in China und in Deutschland kaum ein Unterschied.

\section{Die von den Veranlassern begangenen Rechtsverletzungen}

Der Veranlasser ist der, der die Menschenfleischsuche eingeleitet hat. Er könnte während der Menschenfleischsuche zwei Hauptverhalten zeigen. Das erste Verhalten ist, dass er den anderen Internetnutzer motiviert bzw. erregt hat, an der Suche teilzunehmen. Das zweite ist, dass er die Bildnisse oder die persönliche Information der Zielperson ohne ihre Einwilligung im Internet veröffentlicht hat. Das zweite Verhalten gliche dem Verhalten des Informationsberichters, wenn die Bildnisse oder die persönliche Information der Zielperson zum ersten Mal veröffentlicht würden; wenn die Bildnisse oder die persönliche Information der Zielperson schon veröffentlicht und nur von dem Veranlasser gesammelt würden, gliche sein Verhalten dem des Informationssammlers. Deswegen wird hier nur das erste Verhalten diskutiert.

Der Veranlasser spielt bei der Menschenfleischsuche die wichtigste Rolle. Ohne ihn würde eine Menschenfleischsuche überhaupt nicht stattfinden. Internet als Medium ist stark fragmentiert. ${ }^{385}$ Das heißt, obwohl es im Internet umfangreiche Informationen gibt, sind sie nicht organisiert. Um die Informationen für ein bestimmtes Thema herauszufiltern, muss der Nutzer erst einmal ein "Schlagwort“ haben, um etwas zu suchen. ${ }^{386}$ Der Veranlasser ist derjenige, der das Schlagwort gegeben hat. Ohne seine Erregung ist eine intensive Neugier an einer bestimmten Person unmöglich. Dies ist besonders der Fall, wenn ein Verhalten gar nicht selten auftritt, aber genau das von der Zielperson von dem Veranlasser herausgehoben und ihr angeschuldigt wird. ${ }^{387}$

385 Gounalakis/Klein, NJW 2010, 566, 567.

386 Vgl. Gounalakis/Klein, NJW 2010, 566, 567.

387 Frenz, ZUM 2012, 282, 289. 


\section{In Deutschland}

Der Veranlasser soll als Anstifter haften, wenn er beim Veranlassen die Rechtsverletzung der Zielperson als das Ergebnis der Menschenfleischsuche gewollt hat. Hier spielt die Motivation der Menschenfleischsuche eine große Rolle. ${ }^{388}$ Jedoch ist es in der Praxis schwierig zu beweisen, ob der Veranlasser beim Veranlassen einen Vorsatz hat. Gegebenenfalls könnte aber die Motivation des Veranlassers durch sein Verhalten während der Menschenfleischsuche vermutet werden. Im Fall von „Aufruf zum Lynchmord“ unter $\$ 1$ VII 2 b) hat der Veranlasser durch sein Verhalten beim Veranlassen schon relativ deutlich gemacht, dass eine Rechtsverletzung der Zielperson von ihm gewollt ist.

In den meisten Fällen hat der Veranlasser die Menschenfleischsuche ohne einen bestimmten Vorsatz über die Rechtsverletzung der Zielperson eingeleitet. Von ihm wird es häufig nicht vorausgesehen, dass ein rechtsverletzendes Suchergebnis am Ende der Menschenfleischsuche entstehen würde. Trotz seiner Unschuld könnte der Veranlasser als Störer haften ${ }^{389}$, weil sein Veranlassen tatsächlich kausal zur Rechtsverletzung der Zielperson beitragen hat ${ }^{390}$, und er rechtlich die Möglichkeit zur Verhinderung der Rechtsverletzung hatte ${ }^{391}$, wenn er beim Veranlassen mehr Sorgfalt auf die mögliche Ergebnisse haben würde. Dies ist besonders der Fall, wenn Menschenfleischsuche schon häufig passiert, und auch eine durchschnittliche Person Bescheid weiß oder wissen soll, dass die Suche während des Ablaufs häufig schief geht.

\section{In China}

Weil der Veranlasser die Menschenfleischsuche eingeleitet hat, muss er nach chinesischen Gesetzen für die während Menschenfleischsuche entstehenden Rechtsverletzungen als Anstifter haften, wenn er dafür schuldig wäre. ${ }^{392}$ Die

388 Siehe Oben unter $\$ 1$ III 1.

389 Spindler/Anton in Spindler/Schuster, $\$ 1004$ BGB, Rn. 8; BGHZ 148, 13, 17 = NJW 2001, 3265, 3266; BGH, GRUR 2002, 618, 619; BGHZ 158, 236, 251 = MMR 2004, 668,671 .

390 BGH, GRUR 2004, 860, 864; vgl. BGHZ 148, 13, 17; BGH, GRUR 2002, 618, $619=$ WRP 2002, 532.

391 Spindler/Anton in Spindler/Schuster, $\$ 1004$ BGB, Rn. 8; BGHZ 148, 13, $17=$ NJW 2001, 3265, 3266; BGH, GRUR 2002, 618, 619; BGH, GRUR 1999, 418, 420 = NJW 1999, 1960, 1960; BGHZ 158, 236, 251 = MMR 2004, 668, 671.

392 Gemäß $\$ 148$ OVG-AGZR-Ansichten i.V. mit $\$ 130$ AGZR; vgl. Dai Yingjie, Journal of Zhejiang Institute of Media and Communications 2011, 37, 40; und Yang Lixin, 
Schuldform kann Vorsatz oder Fahrlässigkeit sein. Hätte der Veranlasser die Rechtsverletzung der Zielperson als Ergebnis der Menschenfleischsuche gewollt, muss er als Täter angesehen werden. Ob ein Anstifter auch wegen Fahrlässigkeit haften soll, dazu gibt es in der chinesischen Literatur unterschiedliche Meinungen. ${ }^{393}$ Diese Frage kann meiner Meinung nach durch die systematische Auslegung der bezüglichen Regelungen beantwortet werden.

Gemäß $\$ 148$ OVG-AGZR-Ansichten ist der Anstifter ein Mittäter und muss gesamtschuldnerisch haften. Gemäß $\$ 3$ der „Erklärung einiger Fragen über die Gesetzesanwendung für die Beurteilung der Fälle über den aufgrund von personenbezogenen (körperliche und geistliche) Rechtsverletzungen entstehenden Schadenersatzanspruch“ vom Obersten Volksgericht begründet Mittäterschaft auch wegen gemeinsamer Fahrlässigkeit, oder wenn keine gemeinsame Absicht oder Fahrlässigkeit besteht, aber die rechtsverletzenden Verhalten miteinander unmittelbar kombinieren und zusammen zu einem Schaden der Anderen führen. Aufgrund dieser Regelungen ist Vorsatz keine notwendige Voraussetzung einer Mittäterhaftung. Fahrlässige Anstiftung führt auch zu gesamtschuldnerischen Haftung im Sinne von Mittäterhaftung. Auch wenn man sagt, dass der Anstifter ein Vorsatz haben muss, ist die oben genannte Auslegung kein Widerspruch. Ein „fahrlässige Anstifter" muss nicht unbedingt als Anstifter benannt werden. Allein das fahrlässige anstiftende Verhalten führt schon zur Mittäterhaftung. Vorsätzliche und fahrlässige Anstiftung haben nach chinesischen Gesetzen das gleichen Rechtsfolge. ${ }^{394}$

Bezüglich der Fahrlässigkeit ist zu prüfen, ob der Veranlasser das Ergebnis voraussehen konnte, dass die Menschenfleischsuche zu einer Rechtsverletzung des Betroffenen führen würde. Es ist schon deutlich, dass die Menschenfleischsuche darauf zielt, die Informationen der Zielperson ohne ihre Einwilligung zu erheben und zu veröffentlichen. Das bedeutet, dass Menschenfleischsuche naturgemäß die hohe Gefahr hat, das Persönlichkeitsrecht der Zielperson zu verletzen. ${ }^{395}$ Vor der Einleitung einer Menschenfleischsuche sollte der Veranlasser

Netinfo Security 2008, No. 10, 7, 8; sowie Sun Xiaolin, Science-Technology and Law 2012, No. 3, 84, 86.

393 Wang, Liming, Qin quan xing wei fa yan jiu (Shang juan), Peking 2004, S. 710-712; Yang, Lixin, Qin quan fa lun, 3. Aufl., Peking 2005, S. 601; Shi, Shangkuan, Zhai fa zong lun, Peking 2000, S. 175; Wang, Zejian, Qin quan xing wei fa (Di 2 ce), Taibei 2006, S. 41.

394 Vgl. Wang, Zejian, Qin quan xing wei fa (Di 2 ce), Taibei 2006, S. 41.

395 Li Yifei, Journal of Northwest University (Philosophy and Social Sciences Edition) 2010, No. 5, 67, 70 . 
die Gefahr voraussehen können. ${ }^{396}$ Daraus resultiert die Sorgfaltspflicht des Veranlassers, während der Suche notwendige Maßnahme vorzunehmen, um die Rechtsverletzungen zu vermeiden.

Tatsächlich ist diese Sorgfaltspflicht ein hoher Anspruch an den Veranlasser, der eine Menschenfleischsuche einleitet, weil wie gesagt, die Suche, nachdem sie eingeleitet wurde, schlecht kontrollierbar ist. Jedoch kann eine Rechtsverletzung bei der Offenlegung persönlicher Informationen nicht aufgenommen werden, wenn ein überwiegend zu schützendes Interesse an der Suche bestanden hat. Darum muss der Veranlasser vor der Einleitung einer Menschenfleischsuche abwägen, ob die Suche wegen öffentlichen Interesses oder anderer Interessen gerechtfertigt ist oder nur zu puren Rechtsverletzungen führen würde. Diese Sorgfaltspflicht des Veranlassers ist für eine gesunde Entwicklung der Menschenfleischsuche nützlich, weil dadurch eine Kontrolle an der Quelle aufgebaut ist.

$\mathrm{Zu}$ erwähnen ist hier eine andere Unkontrollierbarkeit der Menschenfleischsuche, nämlich die, dass die Zielperson während der Suche wechselt. Die vom Veranlasser festgelegte Zielperson verliert in diesem Fall die Aufmerksamkeit der Internetnutzer, während ihr Platz von einer anderen mit dem Ereignis bezüglichen Person eingenommen wird. Ob der Veranlasser für die Rechtsverletzung der geänderten Person als Mittäter haftet, soll in jedem einzelnen Fall geprüft werden, ob er für die Änderung der Zielperson Schuld hat. ${ }^{397}$

Neulich hat das Oberste Volksgericht in seiner „Regelung über einigen Fragen hinsichtlich der anzuwendenden Gesetzen bei der Beurteilung von zivilrechtlichen Streitigkeiten wegen Verletzung der persönlichen Rechte und Interesse durch Internet" extra die Rechtsverletzung durch den Veranlasser geregelt. Nach $\$ 15$ dieser Regelung unterstützen die Volksgerichte den Anspruch des Verletzten auf Mittäterhaftung gegen denjenigen, der einen oder mehreren Dritten anstellt, organisiert, anstiftet oder hilft, die persönliche Information des Verletzten zu veröffentlichen oder verbreiten, die zur Verletzung der persönlichen Rechte und Interessen des Verletzten führt. Undeutlich ist aber in dieser Regelung, ob ein Vorsatz vom Anstifter zur Rechtsverletzung der Zielperson bestehen muss, um die Mittäterhaftung des Anstifters zu begründen; oder reicht lediglich eine Kausalität zwischen dem Veranlassen und rechtsverletzendem Ergebnis. Meiner Meinung nach soll $\$ 15$ dieser Regelung in Verbindung mit den

396 Li Yifei, Journal of Northwest University (Philosophy and Social Sciences Edition) 2010, No. 5, 67, 70; Zhou Xiaochen, Netinfo Security 2008, 13, 14.

397 Zhai Xiangjuan, Journal of North China Institute of Water Conservancy and Hydroelectric Power (Social Science) 2010, No. 5, 128, 129; siehe auch Yang Lixin, Netinfo Security 2008, No. 10, 7, 8. 
chinesischen allgemeinen Vorschriften angewendet werden. Das heißt, dass die Schuld des Anstifters bei der Beurteilung der Mittäterhaftung ständig geprüft werden muss. Vom Namen aus kann man sehen, dass die oben genannte Regelung nur zur Erklärung der anzuwendenden Gesetze für bestimmte Situationen dient. Die allgemeinen Prinzipien bleiben bei der Beurteilung der Mittäterhaftung unberührt.

\section{Zwischenergebnis}

In Deutschland wird das Verhalten des Veranlassers im vorsätzlichen Fall durch Mittäterhaftung wegen Anstiftung und im fahrlässigen Fall durch Störerhaftung reguliert. In China gibt es zwar keine Regelung über Störerhaftung, aber zur Begründung einer Mittäterhaftung ist eine sehr niedrige Schwelle gesetzt, sodass auch die gemeinsame Fahrlässigkeit zur Mittäterhaftung führen kann. Die fahrlässige Anstiftung kann in China ebenso eine Mittäterhaftung begründen. Dies hat die Lücke der Störerhaftung in China einigermaßen gefüllt.

\section{Die von den Kommentator begangenen Rechtsverletzungen}

Der Kommentator ist der Internetnutzer, der die Zielperson und ihr Verhalten kommentiert. Deren Kommentierung könnte nur eine Verletzung des Rechts der persönlichen Ehre begründen.

\section{Nach deutschem Recht}

Das Recht der persönlichen Ehre ist eine Ausprägung des allgemeinen Persönlichkeitsrechts, das ein Rahmenrecht ist und keine festgelegte Reichweite hat. ${ }^{398}$ Eine Verletzung des Rechts der persönlichen Ehre ist nur dann begründet, wenn das Interesse der Zielperson die schutzwürdigen Belange des Kommentators überwiegt. ${ }^{399}$ Das heißt, für jeden einzelnen Fall ist eine Interessenabwägung zwischen dem Persönlichkeitsrecht der Zielperson und der Meinungsfreiheit des Kommentators immer notwendig. Prinzipiell haben die beiden Interessen den gleichen Verfassungswert. ${ }^{400}$ Jedoch gibt es nach deutschem Recht schon einige Prinzipien während der Abwägung zu befolgen.

\footnotetext{
398 BGH, NJW 2010, 2728, Rn. 12.

399 BGH, NJW 2010, 2728, Rn. 12.

400 Ballhausen/Roggenkamp, K\&R 2008, 403, 405.
} 


\section{a) Werturteil und meinungsbezogene Tatsachenbehauptung sind grundsätzlich geschützt}

Werturteil und Tatsachenbehauptung sind zwei Bestandteile der Meinungsäußerung. Zur Ausübung der Meinungsfreiheit i.S.v. Art. 5 Abs. 1 Satz 1 GG wird prinzipiell nur das Werturteil geschützt. Dagegen verdient die Äußerung von Tatsachen prinzipiell keinen grundrechtlichen Schutz. Aber das BVerfG hat durch seine Entscheidungen die wörtliche Bedeutung des Gesetzestextes entwickelt. Da Tatsachen regelmäßig Voraussetzung zur Bildung von Meinungen $\operatorname{sind}^{401}$, werden sie auch von der Meinungsfreiheit geschützt, wenn sie meinungsbezogen sind und damit zur Meinungsbildung beitragen. ${ }^{402}$ Gleiches gilt, wenn sich Tatsachenbehauptung und Werturteil vermengen und insgesamt in Form von Stellungnahme, Dafürhalten oder Meinen dargestellt werden. ${ }^{403}$

Das Werturteil und die meinungsbezogene Tatsachenbehauptung werden nur untersagt, wenn die Meinungsäußerung einen besonders schwerwiegenden Eingriff in das Persönlichkeitsrecht bringt, und kein legitimierendes öffentliches Interesse besetzt. ${ }^{404}$ Ein leichter Eingriff in das Persönlichkeitsrecht muss von der Zielperson erduldet werden. ${ }^{405}$

Ein besonders schwerwiegender Eingriff in das Persönlichkeitsrecht liegt vor, wenn z.B. die Äußerung in die Intimsphäre der Zielperson reicht. ${ }^{406}$ Jedoch kann die Äußerung auch für diesen Sonderfall wegen öffentlichen Interesses legitimiert werden. ${ }^{407} \mathrm{Ob}$ das öffentliche Interesse hinreichend ist, muss zwischen dem Persönlichkeitsschaden und dem Interesse an der Äußerung gemäß dem Verhältnismäßigkeitsprinzip abgewägt werden. ${ }^{408}$ Außer Verhältnis steht z.B., wenn eine Äußerung geeignet ist, „eine erhebliche Breitenwirkung zu entfalten und eine besondere Stigmatisierung des Betroffenen nach sich zu ziehen, so dass sie zum Anknüpfungspunkt für eine soziale Ausgrenzung und Isolierung zu werden drohen ${ }^{\text {“409 }}$.

401 BVerfGE 85, 1 = NJW 1992, 1439.

402 OLG Hamburg, CR 2011, 667, 669; siehe auch Ballhausen/Roggenkamp, K\&R 2008, 403, 405; Härting, CR 2009, 21, 22; BVerfGE 54, 208, 219; 61, 1, 8; 65, 1, 41; 85, 1, 15; $90,1,15$.

403 Vgl. BGH, NJW 2008, 2110; BVerfG, NJW-RR 2010, 470.

404 Härting, CR 2009, 21, 22.

405 Vgl. BGH, NJW 2008, 2110; BVerfG, NJW-RR 2010, 470.

406 Härting, CR 2009, 21, 22.

407 BVerfG, NJW 2010, 1587, 1589.

408 BGH, NJW 2010, 2728, Rn. 14.

409 BGH, NJW 2010, 2728, Rn. 14; vgl. BVerfGE 97, 391, 404f. = NJW 1998, 2889; BVerfG, NJW 2009, 3357 = AfP 2009, 365; BGH, NJW-RR 2007, 619, 620. 
Die Beschränkung der Meinungsfreiheit verlangt in Deutschland strenge Voraussetzungen. Das Kommentieren über die Zielperson ist als Werturteil und meinungsbezogene Tatsachenbehauptung grundsätzlich erlaubt.

\section{b) Pure Tatsachenbehauptung wird nicht durch Meinungsfreiheit geschützt}

Werturteil und Tatsachenbehauptung sind nicht immer untrennbar. Die Äußerung, in der es nur um Tatsachenbehauptung geht, fällt nicht zum Schutzbereich der Meinungsfreiheit. Sondern sie wird als persönliche Information oder Privatsphäre geschützt. Die Äußerung purer Tatsachen über die Zielperson begründet auch keine Verletzung des Rechts der persönlichen Ehre, solange die Tatsachen wahr sind.

\section{c) Unwahre meinungsbezogene Tatsachenbehauptung wird nicht geschützt}

Bei Tatsachenbehauptungen gilt die Wahrheitspflicht. ${ }^{410}$ Unwahre Äußerungen sind kein schützenswertes Gut ${ }^{411}$. Dies gilt, wenn die Tatsache zum Zeitpunkt der Äußerung als unwahr erwiesen ist. ${ }^{412}$ Unwahre meinungsbezogene Tatsachenbehauptung könnte sich zur Diffamierung entwickeln, und weiterhin zur Verletzung des Rechts der persönlichen Ehre führen.

Als unwahre Tatsachenbehauptung gilt auch die Äußerung wahrer Tatsachen, die nur teilweise den Lesern mitgeteilt werden, aus denen nur bestimmte wertende Schlussfolgerungen über die Zielperson gezogen werden können, ${ }^{413}$ während die verschwiegenen Tatsachen zur Bildung eines völlig anderen Profils der Zielperson führen würde. ${ }^{414}$

\section{d) Schmähkritik ist gesetzlich verboten}

Die Schmähkritik ist nicht von der Meinungsfreiheit abgedeckt. ${ }^{415}$ Sie begründet regelmäßig eine Verletzung des Rechts der persönlichen Ehre. ${ }^{416}$ Dazu gehören

410 Härting, CR 2009, 21, 22.

411 BVerfGE 54, 208, $219=$ NJW 1980, 2072.

412 BVerfGE 99, 185, 196f. = NJW 1999, 1322; siehe auch BVerfG, NJW 2004, 592.

413 BVerfG, NJW 2010, 1587.

414 BVerfG, NJW 2010, 1587, 1589; vgl. BVerfGE 12, 113, 130f. = NJW 1961, 819; BVerfGE 114, 339, 353f. = NJW 2006, 207; BGH, NJW 2006, 601, 603.

415 Ballhausen/Roggenkamp, K\&R 2008, 403, 409; BVerfGE 61, 1, 8 = NJW 1983, 1415; BVerfGE 99, 185, 197 = NJW 1999, 1322; BGH, NJW-RR 2007, 619, 620.

416 BGH, NJW 2007, 2558. 
auch Formalbeleidigungen, Angriffe auf die Menschenwürde und andere Äußerungen, bei denen es primär um eine Verunglimpfung und nicht mehr um die Auseinandersetzung in der Sache geht. ${ }^{417}$

\section{Nach chinesischem Recht}

In China gelten einige ähnlich Prinzipien wie in Deutschland bezüglich der Beurteilung der Rechtsverletzung der persönlichen Ehre. Die Kommentare des Kommentators begründen gemäß $₫ 8$ „Antwort zu einigen Fragen hinsichtlich der Beurteilung der Fälle über Ehrverletzung “418 eine Rechtsverletzung der persönlichen Ehre, wenn der Kommentar beleidigten Inhalt enthält, oder wenn der Kommentar eine unwahre Tatsache über die Zielperson erzählt. ${ }^{419}$ Aber alle Rechtsverletzungen der persönlichen Ehre in China haben eine notwendige Voraussetzung, nämlich die Tatsache, dass die Ehre tatsächlich beschädigt ist. ${ }^{420}$

Die Ehre wäre tatsächlich beschädigt, wenn die soziale Bewertung über die Zielperson gemindert wäre. ${ }^{421}$ Die soziale Bewertung ist die Bewertung der Mitmenschen. Das subjektive Gefühl der Zielperson spielt dabei keine Rolle. ${ }^{422}$

Für die Beschränkung der Meinungsfreiheit haben China und Deutschland beide relativ strenge Voraussetzungen entwickelt. Kommentare über die Zielperson von dem Kommentator sind regelmäßig erlaubt, solange sie sich nicht um unwahre Tatsachen oder Schmähkritik handeln.

\section{Interessenabwägungen}

Durch die oben geführte Analyse ist es nicht schwierig zu erkennen, dass die Interessenabwägung für die Beurteilung einer Rechtsverletzung in allen Fällen wesentlich und entscheidend ist. Jedoch ist die Entwicklung einer universalen

417 Schulze-Fielitz in: Dreier, Art. 5 I, II Rn. 50.

418 Vom chinesischen Obersten Volksgerichtshof.

419 Ob die Tatsachenbehauptung um wahre Tatsache handelt, kommt darauf an, ob der Äußernde an der Wahrheit seiner Äußerung glaubt. Siehe oben unter $₫ 6$ II.

420 Siehe oben unter $\$ 6$ II.

421 Zhongshan Mitteleres Volksgericht, Urt. v. 25.11.2005 - (2005) zhong zhong fa min yi zhong zi di 1003 hao; Kaifeng Mitteleres Volksgericht, Urt. v. 19.12.2011 - (2011) bian min zhong zi di 1165 hao; Xi' an Mitteleres Volksgericht, Urt. v. 30.11.2012 - (2012) xi min er zhong zi di 02249 hao; Pukou Unteres Volksgericht, Urt. v. 12.12.2012 - (2012) pu min chu zi di 2125 hao; Jinhua Mitteleres Volksgericht, Urt. v. 26.03.2012 - (2012) zhe jin min zhong zi di 324 hao; Peking Oberes Volksgericht, Urt. v. 29.11.2007 (2007) gao min zhong zi di 1146 hao.

422 Siehe oben unter $\$ 6$ II. 
Regel über Interessenabwägung für alle Fälle unmöglich, weil die Abwägung in jedem einzelnen Fall unter Berücksichtigung der Konstellation durchgeführt werden muss. ${ }^{423}$ Aber es gibt doch einige Prinzipien, die man für ähnliche Sachverhalte verwenden kann. Im Folgenden wird versucht, durch die Untersuchung der Rechtsprechung von ähnlichen Fallgruppen in beiden Ländern, einige grundlegende Prinzipien für bestimmten Sachverhalt zu bestimmen.

Bei der Interessenabwägung müssen alle kollidierenden Rechte und Interessen berücksichtigt werden. Bei der Menschenfleischsuche auftretende Interessen sind vor allem die Meinungsfreiheit der Internetnutzer, die Persönlichkeitsrechte der Zielperson, und das Informationsinteresse der Öffentlichkeit an den Informationen über die Zielperson.

\section{Ein Überblick auf die zu diskutierenden Fallgruppen}

In dieser Arbeit sind fünf Fallgruppen zur Diskussion ausgewählt.

Die erste Fallgruppe betrifft die Prominenten. Bei den Prominenten besteht häufig überwiegendes öffentliches Interesse, das die Persönlichkeitsrechte der Prominenten beschränken könnte. Deswegen ist es häufig auch erlaubt, über die Prominenten zu diskutieren. Gleichzeitig entsteht dadurch die hohe Gefahr, die Persönlichkeitsrechte der Prominenten zu verletzen. Die Diskussion über sie, eine der häufigsten Zielperson der Menschenfleischsuche, ist notwendig und repräsentativ.

Die zweite Fallgruppe betrifft die Personen aus einem Ereignis. Ähnlich wie Prominente sind solche Personen auch irgendwie bekannt geworden. Der Unterschied liegt darin, dass solche Personen häufig passiv an dem Ereignis teilgenommen und unfreiwillig die Aufmerksamkeit der Öffentlichkeit gewonnen haben. Es besteht die Möglichkeit, dass das auf dem Ereignis liegende öffentliche Interesse sich auf die an dem Ereignis bezüglichen Personen erstreckt. Diese Fallgruppe bezieht sich auf den üblichsten Fall der Menschenfleischsuche, weil die Zielperson häufig eine normale unbekannte Person ist, und erst aufgrund einer Menschenfleischsuche bekannt wird.

Die nächste Fallgruppe betrifft die Personen mit besonderem Beruf. Solche Personen sind zwar nicht prominent. Aber an dem von ihnen ausgeübten Beruf besteht häufig überwiegendes öffentliches Interesse, damit eine öffentliche Diskussion über ihrer Berufsausübung bzw. über die Person selbst rechtfertigt. Aufgrund des besonderen Berufs muss eine solche Person wie ein Prominenter auf einen Teil seiner Privatsphäre verzichten. $\mathrm{Zu}$ diesen Berufen gehören z.B. Lehrer, Professor, Arzt usw.

423 Vgl. Ballhausen/Roggenkamp, K\&R 2008, 403, 405. 
Eine Diskussion könnte auch über Straftäter erfolgen. Wegen des rechtswidrigen Verhaltens ist der Straftäter ins Blickfeld der Öffentlichkeit getreten. Nicht nur bezüglich seines Verhaltens sondern auch bezüglich der Person des Straftäters besteht häufig ein überwiegendes öffentliches Interesse. Die Diskussion über diese Fallgruppe bietet weiterhin die Möglichkeit eines Vergleichs mit der Menschenfleischsuche wegen des rechts- und moralwidrigen Verhaltens der Zielperson, das noch nicht strafbar ist.

Ebenso wie der Straftäter werden die persönlichen Informationen der Parteien als Teilnehmer an dem Gerichtsprozess und als Adressaten des gerichtlichen Urteils durch die Entscheidung offengelegt. Wegen der Öffentlichkeit der Gerichtsverhandlung ist es möglich, dass die persönlichen Informationen der Parteien allgemein zugänglich werden. Wegen Neugier könnten die Parteien von einem umstrittenen Urteil Zielperson der Menschenfleischsuche werden. Der Schutz des Persönlichkeitsrechts der Parteien des Gerichtsprozesses wird hier diskutiert.

\section{Die Interessenabwägung im Fall der Verletzung des Persönlichkeitsrechts der Prominenten}

\section{a) Nach deutschem Recht}

Vor 2004 wurden die Prominenten „absolute Personen der Zeitgeschichte“ genannt. Als absolute Personen der Zeitgeschichte bezeichnete man die Personen, "die aufgrund ihrer hervorgehobenen Stellung in Staat und Gesellschaft oder durch außergewöhnliches Verhalten oder besondere Leistungen aus der Masse der Mitmenschen herausragen “424, und dauerhaft im Blickpunkt der Öffentlichkeit stehen. Zu den absoluten Personen der Zeitgeschichte gehörten nach der Rechtsprechung Angehörige fürstlicher Häuser, Staatsoberhäupter und Politiker, Vertreter von Wissenschaft und Forschung, Vertreter der Wirtschaft, Künstler insbesondere Schauspieler und Spitzensportler. ${ }^{425}$

Die „absoluten Personen der Zeitgeschichte“ wird mit den „relativen Personen der Zeitgeschichte" unterschieden, die durch ein spektakuläres Ereignis für begrenzte Zeit ins Blickfeld der Medien geraten sind. ${ }^{426}$ Verliert das Ereignis an Interesse, kehrt der Betroffene auch wieder in die Anonymität zurück. Die Interessenabwägung über „die relativen Personen der Zeitgeschichte“ wird in der nächsten Fallgruppe diskutiert.

424 Fricke in Wandtke/Bullinger, KunstUrhG $\$ 23$, Rn. 8.

425 Damm/Rehbock, 1. Teil, Rn. 211.

426 Damm/Rehbock, 1. Teil, Rn. 225. 
Die Rechtsfiguren der „absoluten und relativen Personen der Zeitgeschichte" sind wegen der ersten Carolinen-Entscheidung vom BVerfG im Jahr 2001 in Frage gestellt worden, ${ }^{427}$ und auf sie wurde unter dem Druck des EGMR ${ }^{428}$ vom BGH verzichtet. ${ }^{429}$ Der EGMR hat durch den Fall "Caroline von Hannover“ entgegen dem BVerfG ${ }^{430}$ ein Prinzip zum Schutz der Prominenten entwickelt, dass sie als Personen des öffentlichen Lebens auch eine vor der Öffentlichkeit geschützte Privatsphäre als Teil des allgemeinen Persönlichkeitsrechts benötigen. ${ }^{431}$ Aufgrund dieses Prinzips darf über rein private Aktivitäten der Prominenten prinzipiell nicht mehr berichtet werden. ${ }^{432}$ Ein Sonderfall wäre es nur, wenn die Veröffentlichung zu einer Debatte von allgemeinem Interesse beiträgt. ${ }^{433}$

Mit dem Verzicht des Begriffs von absoluten und relativen Personen der Zeitgeschichte werden die Prominenten besser vor den öffentlichen Diskussionen geschützt. Nach der aktuellsten Rechtsprechung rechtfertigt es nicht mehr, allein aus dem Faktum der Prominenz oder öffentlichen Bekanntheit der Zielperson sie unter Menschenfleischsuche zu stellen oder seine persönlichen Informationen willkürlich offenzulegen. ${ }^{434}$ Zur „bloßen Unterhaltung“ oder um die Neugier $\mathrm{zu}$ befriedigen ist demnach ebenso nicht ausreichend, um eine Suche oder eine Veröffentlichung zu begründen. ${ }^{435}$ Eine Menschenfleischsuche nach Prominenten kann nach deutschem Recht nur begründet werden, wenn sie mit einem Beitrag zu einer Diskussion mit allgemeinem Interesse verknüpft wird. ${ }^{436}$

„Beitrag zu einer Debatte von allgemeinem Interesse“ als ein entscheidender Maßstab hat nicht unbedingt zu Erleichterung in der Praxis geführt, ${ }^{437}$ weil das allgemeine Interesse als Begriff in Deutschland sehr abstrakt und umfassend ist. ${ }^{438}$ In der Praxis ist es relativ einfach, irgendeine Verknüpfung zwischen einem Thema über einen Prominenten und dem allgemeinen Interesse zu finden, obwohl

\footnotetext{
427 BVerfG, NJW 2001, 1921, 1922.

428 EMGR, NJW 2004, 2647.

429 Wanckel, NJW 2011, 726.

430 BVerfGE 101, 361 = NJW 2000, 1021.

431 Frenz, NJW 2012, 1039.

432 Frenz, NJW 2012, 1039.

433 Frenz, NJW 2012, 1039.

434 BVerfG, NZV 2006, 521; vgl. BVerfG, NJW 2001, 1921, 1922; vgl. auch Schertz in Loewenheim, \$18, Rn. 31.

435 Frenz, NJW 2012, 1039, 1040.

436 Frenz, NJW 2012, 1039, 1040.

437 BVerfG, NZV 2006, 521; vgl. BVerfG, NJW 2003, 3262; siehe auch Frenz, NJW 2012, 1039, 1040.

438 Frenz, NJW 2012, 1039, 1040.
} 
der Zusammenhang sehr locker sein könnte. ${ }^{439}$ Im Fall der Menschenfleischsuche besteht dann die Gefahr, dass die Teilnehmer der Menschenfleischsuche das allgemeine Interesse als Vorwand benutzen, um ihr Verhalten während der Menschenfleischsuche zu rechtfertigen. ${ }^{440}$

Der EGMR hat das Problem auch aufgespürt, dass man die Verknüpfung zwischen einem Thema und dem allgemeinen Interesse leicht umgehen kann. Als Lösung hat er auf die Möglichkeit verwiesen, solche Fälle im Verfahren vor geeignete nationale Gerichte zu bringen. ${ }^{441}$ In Deutschland kann das BVerfG für jeden Fall prüfen, ob die Verknüpfung als Beitrag zur Bildung der öffentlichen Meinung zu werten ist, so dass der Schutz des Persönlichkeitsrechts überwiegt ${ }^{442}$.

Klar ist jedoch, dass die bezügliche Äußerung über den Prominenten nicht zur Schaffung eines „Klimas ständiger Belästigung“ führen darf. ${ }^{443}$

\section{b) Nach chinesischem Recht}

In der Literatur in China ist nur ein einziges Merkmal zur Definition der Prominenten betont, nämlich die „hohe Publizität“. Alle Stars aus dem Kultur- und Sportbereich, die Leiter von Organisationen des öffentlichen Interesses, Wissenschaftler, Beamte, Präsidenten und Könige, Kriegsverbrecher, Drogenschmuggler und Leiter der terroristischen Organisationen gehören zu Prominenten, solange sie hohe Publizität haben. ${ }^{444}$

Der Prominente in China wird auch als „öffentliche Figur" bezeichnet. Der Begriff wurde im Jahr 2002 im Fall von „Fan Zhiyi \& Wenhui-xinmin United Press Group" von einem unteren Volksgericht in Shanghai das erste Mal verwendet. ${ }^{445}$ In diesem Fall hat das Gericht ein Kriterium für die Beurteilung der Verletzung des Persönlichkeitsrecht der Prominenten entwickelt: die Privatsphäre oder die persönliche Ehre der Prominenten würde als öffentlichem Interesse bezüglich angesehen und den Schutzbereich der privaten Angelegenheit ${ }^{446}$

\footnotetext{
439 Frenz, NJW 2012, 1039, 1040.

440 Frenz, NJW 2012, 1039, 1040.

441 Frenz, NJW 2012, 1039, 1040.

442 EGMR, NJW 2012, 1053, Rn. 119.

443 EGMR, NJW 2012, 1053, Rn. 117 f., 122 f.

444 Wang Liming/Ge Weibao, S. 9; siehe auch Zhang Xinbao, Legal protection of the right to privacy, S. 17.

445 Jingan Unteres Volksgericht, Urt. v. 18.09.2002 - (2002) jing min yi (min) chu zi di 1776 hao.

446 „Der Schutzbereich der privaten Angelegenheit“ ist eine direkte Übersetzung der Text der Entscheidung. Es bedeutet nämlich „der Schutzbereich des Persönlichkeitsrechts“.
} 
verlassen, wenn sie mit einem Ereignis verknüpft ist, auf dem die Öffentlichkeit fokussiert ist; dies ist besonders der Fall, wenn das Ereignis umfangreiches Interesse und allgemeine Aufmerksamkeit der Öffentlichkeit und der Medien aufweckt. ${ }^{447}$

Außer von dem öffentlichen Interesse kann das Persönlichkeitsrecht der Prominenten nach der Meinung in der Literatur auch aufgrund staatlicher Interessen und dem öffentlichen Auskunftsrecht zurücktreten. ${ }^{48}$ Ohne die oben genannten Interessen dürfen die persönlichen Informationen der Prominenten nicht während der Menschenfleischsuche verbreitet und ggf. diskutiert werden.

Im Jahr 2005 gab es in China ein Ereignis, dass die wahren privaten Telefonnummern von 600 chinesischen Prominenten im Internet veröffentlicht wurden. ${ }^{449}$ Die betroffenen Prominenten hatten ständig fremde Anrufe bekommen und mussten die Telefonnummer wechseln. Obwohl keiner von den Prominenten deswegen eine Klage eingereicht hatte, ist es in der Diskussion über dieses Ereignis allgemein akzeptiert, dass die Offenlegung ihrer privaten Telefonnummern offensichtlich eine Rechtsverletzung ihrer Privatsphäre begründet. ${ }^{450}$

Wie in Deutschland ist es in China auch allgemein anerkannt und von der Entscheidungspraxis festgelegt, dass die Grenze der öffentlichen Diskussion über Prominente an der Schmähkritik liegt. ${ }^{451}$ Beleidigung oder Diffamierung ist auch gegen Prominente nicht erlaubt.

\section{Die Interessenabwägung im Fall der Verletzung des Persönlichkeitsrechts der Person aus einem Ereignis}

\section{a) Nach deutschem Recht}

Wie bereits ausgeführt wurde, wurde die Person, die bisher allgemein überhaupt nicht bekannt ${ }^{452}$ und durch ein spektakuläres Ereignis ins Blickfeld der

447 Jingan Unteres Volksgericht, Urt. v. 18.09.2002 - (2002) jing min yi (min) chu zi di 1776 hao.

448 Li Yifei, Journal of Northwest University (Philosophy and Social Sciences Edition) 2010, No. 5, 67, 69.

449 Chen Zhong, Da yang wang, 06.06.2005, http://it.sohu.com/20050606/n240093336. shtml (besucht am 04.04.2015).

450 Zhang Xuejun/Wang Fei/Guo Zhixia, Bei jing yu le xin bao, 05.06.2005, http:// news.xinhuanet.com/newmedia/2005-06/05/content_3045908.htm (besucht am 04.04.2015).

451 Peking Oberes Volksgericht, Urt. v. 29.11.2007 - (2007) gao min zhong zi di 1146 hao.

452 Frenz, ZUM 2012, 282, 284. 
Öffentlichkeit getreten ist, früher als relative Person der Zeitgeschichte bezeichnet. Der Begriff von absoluter und relativer Person der Zeitgeschichte wird zwar nicht mehr verwendet; aber das Prinzip für relative Personen der Zeitgeschichte ist immer noch relevant, weil durch das Verzichten des Begriffs das Schutzniveau für die absolute Person der Zeitgeschichte auf das Schutzniveau der relativen Person angehoben wurde, während der Schutz der relative Person unverändert bleibt. ${ }^{453}$

Eine Beschränkung des Persönlichkeitsrechts der Person aus einem Ereignis ist möglich, wenn ein hinreichendes öffentliches Interesse an dem Ereignis gegeben ist. Die Person ist durch das Ereignis bekannt geworden. Deswegen sollen die Offenlegung ihrer persönlichen Informationen und die Diskussion über sie auch in unmittelbaren Zusammenhang mit dem Ereignis bleiben. Diese Meinung wurde durch die Rechtsprechung vom BVerfG veröffentlicht.

In einer Rechtsprechung über die Veröffentlichung der Lichtbilder einer Person in einem Geschehen hat das BVerfG festgestellt, dass die zu veröffentlichenden Lichtbilder einen unmittelbaren Zusammenhang mit der Rolle, die die Person in diesem Geschehen spielt, haben müssen. ${ }^{454}$ Die Veröffentlichung der Lichtbilder, die „einen über diese Thematik hinausreichenden Aufschluss über das Privatleben der Betroffenen eröffnen", ist rechtswidrig. ${ }^{455}$

Mit dieser Denkweise kann man auch den Persönlichkeitsrechtsschutz der Zielperson von der Menschenfleischsuche untersuchen, die wegen des rechtsoder moralwidrigen Verhaltens der Zielperson durchgeführt wird. In diesem Fall begründet das Verhalten der Zielperson das Ereignis. Bestände auf dem Ereignis umfangreiches Interesse und allgemeine Aufmerksamkeit der Öffentlichkeit, dürfen die persönlichen Informationen während der Menschenfleischsuche erörtert werden, die einen unmittelbaren Zusammenhang mit dem rechts- oder moralwidrigen Verhalten der Zielperson haben.

Mit der Person aus einem Ereignis zu unterscheiden ist die Person, die bewusst die „öffentliche Arena“ betreten hat. ${ }^{456}$ Dies wäre nach der Meinung des EGMR der Fall, wenn eine Person mit Absicht an einer öffentlichen Diskussion teilnimmt. ${ }^{457}$ Für diese Person gelten „die besonderen Maßstäbe eines gesteigerten Öffentlichkeitsbezugs und damit einer erhöhten Sozialbindung des

453 Vgl. Schertz in Loewenheim, $\$ 18$, Rn. 31.

454 BVerfG, NJW 2006, 3406, 3408.

455 BVerfG, NJW 2006, 3406, 3408.

456 Frenz, ZUM 2012, 282, 283.

457 Frenz, ZUM 2012, 282, 283. 
Individuums “ ${ }^{458}$ weil sie durch das Betreten in der öffentlichen Arena ihre Privatsphäre einigermaßen eröffnet hat.

\section{b) Nach chinesischem Recht}

Um die Person aus dem Ereignis zu beschreiben gibt es in China den Begriff des "relativen Prominenten“459 oder „unfreiwilligen Prominenten “460.Wie in Deutschland ist sie bekannt geworden, entweder weil sie einem Ereignis bezüglich ist, das das öffentliche Interesse aufgeweckt hat, ${ }^{461}$ oder weil ihr Verhalten selbst zu einem Ereignis führt. Ihre Bekanntschaft beschränkt sich jedoch auf eine bestimmte Zeit oder einen bestimmten Ort. ${ }^{462}$

Es herrscht in chinesischer Literatur auch die Meinung, dass die Persönlichkeitsrechte der „unfreiwilligen Prominenten“ einigermaßen beschränkt werden sollen, weil sie tatsächlich Verbindung mit dem öffentlichen sozialen Leben geschlossen haben und Objekt des öffentlichen Interesses geworden sind. ${ }^{463}$ Der wirkliche Wille der Personen, ob sie bewusst an dem Ereignis teilnehmen wollten, spielt hier keine Rolle.

In China ist es allgemein anerkannt, dass das Recht auf Privatsphäre für jede Person aufgrund des öffentlichen Interesses einigermaßen einschränkt werden kann. ${ }^{464}$ Aber öffentliches Interesse ist kein eindeutiger Begriff. ${ }^{465}$ Es ist zu vermeiden, das öffentliche Interesse als Vorwand zu benutzen, um die Persönlichkeitsrechte der Person unverhältnismäßig einzuschränken. Besonders zu vermeiden ist es, das öffentliche Interesse als das Interesse der Mehrheit auszulegen. Es ist unrichtig, das Recht einer bestimmen Person zu verletzen, um die

458 Frenz, ZUM 2012, 282, 283.

459 Xiao Han, South Reviews 2003, No. 4, 32, 34.

460 Weng, Guomin/Wang, Chenghong, Journal of Zhejiang Universtity (Humanities and Social Sciences) 2002, No. 2, 33, 37.

461 Weng, Guomin/Wang, Chenghong, Journal of Zhejiang Universtity (Humanities and Social Sciences) 2002, No. 2, 33, 37.

462 Li Yifei, Journal of Northwest University (Philosophy and Social Sciences Edition) 2010, No. 5, 67, 69.

463 Weng, Guomin/Wang, Chenghong, Journal of Zhejiang Universtity (Humanities and Social Sciences) 2002, No. 2, 33, 37.

464 Li Yifei, Journal of Northwest University (Philosophy and Social Sciences Edition) 2010, No. 5, 67, 69; vgl. Zhang Di, S. 24; Qin Qianhong/Li Gaoya, Study and Practice 2011, No. 11, 57, 58; Zhang Zuoguo, Western Law Review 2009, No. 6, 7, 12.

465 Hu Dan, S. 28. 
Interessen der Mehrheit zu verwirklichen. Das Leiden der Minderheit und die Zufriedenheit der Mehrheit kann nicht gegenseitig abgewogen werden. ${ }^{466}$

\section{Die Interessenabwägung im Fall der Verletzung des Persönlichkeitsrechts der Person mit besonderen Beruf}

\section{a) Nach deutschem Recht}

Berufsleben gehört häufig zur Sozialsphäre. Als Sozialsphäre kennzeichnet man den durch die Kommunikation und Interaktion des Einzelnen mit den anderen in der Gemeinschaft zusammen lebenden Bürger. ${ }^{467}$ Bei der Ausübung der Berufstätigkeit wirkt man durch sein Verhalten auf andere ein und berührt dadurch die persönliche Sphäre seiner Mitmenschen und Belange des Gemeinschaftslebens. ${ }^{468}$ Dieser Sozialbezug hat zur Folge, dass das Persönlichkeitsrecht eingeschränkt ist, wenn die bezügliche berufliche Leistung in wertender Art und Weise berichtet wird. ${ }^{469}$

Das bedeutet aber nicht, dass die Einschränkung des Persönlichkeitsrechts für jeden Beruf gleich ist. ${ }^{470} \mathrm{Um}$ eine rechtmäßige Einschränkung zu begründen, muss ein entsprechendes Informationsinteresse der Öffentlichkeit bestehen. ${ }^{471}$ Nicht jede berufliche Tätigkeit steht im Fokus der Öffentlichkeit ${ }^{472}$. Das Informationsinteresse der Öffentlichkeit kommt auf die Wirkung der beruflichen Tätigkeit für die Mitmenschen an. ${ }^{473}$ Je herausragender und öffentlicher die Position des Betroffenen ist, desto mehr hat er die Veröffentlichung seiner persönlichen Informationen hinzunehmen. ${ }^{474}$

Dies gilt auch für die wertende Laienberichterstattung im Rahmen von Bewertungsplattformen, ${ }^{475}$ auf der der Betroffene und seine berufliche Tätigkeit mit Namensnennung bewertet sind. Geht es um eine unmittelbare personenbezogene Bewertung, steht der Schutz der Meinungsfreiheit normalerweise hinter

$466 \mathrm{Wu}, \mathrm{Bo}, \mathrm{S} .8$.

467 Vgl. BGH, NJW-RR 2007, 619, 620.

468 Ballhausen/Roggenkamp, K\&R 2008, 403, 405.

469 Ballhausen/Roggenkamp, K\&R 2008, 403, 405; vgl. BVerfGE 35, 202, 220; BVerfGE 97, 391, 406; BVerfG, NJW 2000, 2413, 2414; BVerfG, NJW 2003, 1109; BGH, NJWRR 2007, 619, 620.

470 Ballhausen/Roggenkamp, K\&R 2008, 403, 405.

471 Ballhausen/Roggenkamp, K\&R 2008, 403, 405; vgl. BGH, NJW-RR 2007, 619, 620.

472 Ballhausen/Roggenkamp, K\&R 2008, 403, 405.

473 BGH, VersR 1981, 384, 385; BGH, NJW-RR 2007, 619, 620.

474 Vgl. BGH, NJW-RR 2007, 619, 620.

475 Ballhausen/Roggenkamp, K\&R 2008, 403, 405. 
dem Allgemeinen Persönlichkeitsrecht zurück. ${ }^{476}$ Aber wenn die Bewertung nur berufsbezüglich ist und sich auf die von der Person erbrachte Dienstleistung richtet, wird die Meinungsfreiheit regelmäßig mehr geschützt. ${ }^{477}$

$\mathrm{Zu}$ erwähnen ist, dass die Meinungsfreiheit nicht nur unter dem Vorbehalt des öffentlichen Interesses geschützt wäre. ${ }^{478}$ Art. 5 Abs. 1 GG gewährleistet die Meinungsfreiheit primär in Form von Selbstbestimmung des einzelnen Grundrechtsträgers über die Entfaltung seiner Persönlichkeit in der Kommunikation mit anderen. ${ }^{479}$ Solange der Äußernde überwiegendes Interesse an der Meinungsäußerung hat, wird er geschützt, auch wenn das Thema andere kaum interessiert.

Es ist zu erwarten, dass die „Online-Evaluation“ in der Zukunft auf mehr Berufsstände ausgeübt wird. ${ }^{480}$ Bisher gibt es nur beschränkte Berufe, deren Bewertung durch die Rechtsprechung zugesagt ist.

Das OLG Frankfurt ermöglichte die Bewertung vom Arzt im Internet aufgrund des Rechts auf freie Arztwahl und zwischen Ärzten bestehenden Wettbewerbs. ${ }^{481}$ Das Gericht meinte, die Ärzte arbeiten nicht in einem geschlossenen, abgrenzbaren Raum; es besteht an der Leistung der Ärzte ein geographisch umfassendes öffentliches Interesse. ${ }^{482}$

Der BGH hat in seiner "spickmich.de-Entscheidung" die Bewertung von Lehrern und Professoren auch erlaubt. Nicht nur Schüler, Studenten, aber auch Eltern und Vorgesetzte der Schule oder Universität haben ein überwiegendes Informationsinteresse an den Informationen darüber, wie Lernende die Leistung der Lehrenden einschätzen. ${ }^{483}$ Der Eingriff in die Persönlichkeitsrechte der betroffenen Lehrer und Professoren bezog sich im Wesentlichen auf die Sozialsphäre und in geringem Umfang auf die Privatsphäre. ${ }^{484}$

Aber selbst bei einem zulässigen Fall muss stets beachtet werden, ob die Veröffentlichung persönlicher Informationen und die öffentliche Bewertung „schwerwiegende Auswirkungen auf das Persönlichkeitsrecht z.B. in Form von

476 Ballhausen/Roggenkamp, K\&R 2008, 403, 405.

477 Ballhausen/Roggenkamp, K\&R 2008, 403, 405.

478 BVerfG, NJW 2010, 1587.

479 BVerfG, NJW 2010, 1587.

480 Gounalakis/Klein, NJW 2010, 566.

481 OLG Frankfurt, CR 2012, 399, 400.

482 OLG Frankfurt, CR 2012, 399, 400.

483 Härting, CR 2009, 21, 22.

484 Härting, CR 2009, 21, 22. 
Stigmatisierung, sozialer Ausgrenzung und ganz allgemein in Form einer Prangerwirkung" haben können. ${ }^{485}$

\section{b) Nach chinesischem Recht}

Die Diskussion über die Verletzung der Persönlichkeitsrechte der Person mit besonderem Beruf ist in China selten. Der Grund könnte daran liegen, dass der Begriff von Prominenten oder öffentlichen Figuren sehr breit ausgelegt ist. Alle Personen mit hoher Publizität können als Prominente behandelt werden. Durch die schnelle Verbreitung der Informationen im Internet ist es heutzutage sehr leicht, dass eine Person in kurzer Zeit hohe Publizität bekommt.

Es kann deswegen sein, dass die Person mit besonderem Beruf als Prominenter angesehen wird. In einer Entscheidung von einem unteren Volksgericht in Chongqing z.B. wurde ein Professor als Prominenter behandelt. ${ }^{486}$ Eine Diskussion über ihn und seine akademische Fähigkeit wurde dementsprechend unter Meinungsfreiheit geschützt. ${ }^{487}$

Jedoch sollte die Person mit besonderem Beruf strenger als ein Prominenter geschützt werden, weil das öffentliche Interesse meistens nur auf den Informationen der Person liegt, die mit der beruflichen Tätigkeit unmittelbar bezüglich sind, während alle persönliche Informationen eines Prominenten offengelegt werden dürfen, solange die Veröffentlichung zu einer Debatte von allgemeinem Interesse beiträgt.

Diskussionen über dieses Thema sind in China zu erwarten.

\section{Die Interessenabwägung im Fall der Verletzung des Persönlichkeitsrechts des Straftäters}

Der Straftäter hier bezieht sich ausschließlich auf den vom Gericht verurteilten Straftäter.

\section{a) Nach deutschem Recht}

Ohne Zweifel stellt eine Veröffentlichung der Informationen „über eine Straftat unter Namensnennung, Abbildung oder Darstellung des Täters regelmäßig eine erhebliche Beeinträchtigung des Persönlichkeitsrechts des Täters dar“, „weil sein

485 Vgl. BVerfGE 35, 202, 234 f.; BVerfGE 97, 391, 406 f.; BVerfG, NJW 2000, 2413, 2414; BGH, NJW-RR 2007, 619, 620.

486 Shapingba Unteres Volksgericht, Urt. v. 17.9.2009 - (2009) sha fa min chu zi di 1800 hao.

487 Shapingba Unteres Volksgericht, Urt. v. 17.9.2009 - (2009) sha fa min chu zi di 1800 hao 
Fehlverhalten öffentlich bekannt gemacht und seine Person in den Augen des Publikums negativ qualifiziert werde“ ${ }^{488}$

Aber die Berichterstattungen über Straftat und Straftäter werden trotzdem regelmäßig in den Medien gesehen. Der Grundgedanke liegt darin, dass derjenige, der durch eine Straftat den Rechtsfrieden bricht, sich nicht nur den hierfür verhängten strafrechtlichen Sanktionen beugen muss, ${ }^{489}$ sondern grundsätzlich auch dulden muss, dass „das von ihm selbst durch seine Tat erregte Informationsinteresse der Öffentlichkeit in einer nach dem Prinzip freier Kommunikation lebenden Gemeinschaft auf den dafür üblichen Wegen befriedigt wird“ ${ }^{490}$ „Die Verletzung der Rechtsordnung, die Beeinträchtigung individueller Rechtsgüter, die Sympathie mit den Opfern, die Furcht vor Wiederholungen solcher Straftaten und das Bestreben, dem vorzubeugen ${ }^{\text {"491, }}$, haben insgesamt das öffentliche Interesse begründet.

Einer Straftat steht naturgemäß ein starkes öffentliches Interesse an den Informationen über Tat und Täter gegenüber ${ }^{492}$ Das bedeutet aber nicht, dass über jede Straftat mit Namensnennung des Täters bedingungslos berichtet werden darf. Eine Interessenabwägung ist unter jeder einzelner Situation immer notwendig. Zwei Elemente müssen hier bei der Abwägung berücksichtigt werden.

\section{aa) Die Außergewöhnlichkeit}

Je schlimmer bzw. je außergewöhnlicher die Straftat ist, desto höher ist das öffentliche Interesse daran. ${ }^{493}$ Es liegt z.B. immer ein überwiegendes öffentliches Interesse bei schwerer Kriminalität. ${ }^{494}$ Bei solchen Verbrechen ist in der Regel eine bloße Neugier und Sensationslust schon ausreichend, um die Veröffentlichung näherer Informationen über die Tat und ihren Hergang, über die Person des Täters und seine Motive sowie über die Strafverfolgung zu rechtfertigen. ${ }^{495}$

\footnotetext{
488 BGH, NJW 2006, 599, Rn. 7.

489 BGH, NJW 2006, 599, 600, Rn. 14.

490 BGH, NJW 2006, 599, 600, Rn. 14.

491 BGH, NJW 2010, 2728, Rn. 15.

492 BGH, NJW 2010, 2728, Rn. 15.

493 BGH, NJW 2010, 2728, Rn. 15.

494 BGH, NJW 2006, 599, Rn. 13.

495 BGH, NJW 2010, 2728, Rn. 15; vgl. BVerfGE 35, 202, 231 = NJW 1973, 1226: BVerfG, NJW 2009, 3357 = AfP 2009, 365, Rn. 18; vgl. auch BGHZ 143, 199, $204=$ NJW 2000, 1036.
} 
Eine Einschränkung des Persönlichkeitsrechts des Straftäters kann nach der Auffassung des BGH auch in Fällen von kleiner oder mittlerer Kriminalität gerechtfertigt sein, wenn wegen der Person des Täters, der Stellung der Person des Beschuldigten und der Art der Straftat oder des Tathergangs ein besonderes Informationsinteresse besteht. ${ }^{496}$ Diese Auffassung ist auch vom BVerfG anerkannt. ${ }^{497}$

Außerdem ist eine identifizierende (mit Namensnennung) Berichterstattung und Diskussion über Straftaten (oder sogar nicht unerhebliche Ordnungswidrigkeiten) auch geeignet und zulässig, wenn sie darauf abzielt, Ideen und Informationen zu Fragen von allgemeinem Interesse zu vermitteln und eine Diskussion hierüber in der Gesellschaft auszulösen oder zu bereichern. ${ }^{498}$

Aber das öffentliche Interesse an Straftat und Straftäter steht nicht immer über dem Interesse vom Schutz des Persönlichkeitsrechts des Straftäters. Eine Veröffentlichung seiner persönlichen Informationen und eine identifizierende Bewertung über ihn sind jedenfalls unzulässig, wenn sie dazu führen, „eine erheblich neue oder zusätzliche Beeinträchtigung des Täters zu bewirken und insbesondere seine Wiedereingliederung in die zivilisierte Gesellschaft zu gefährden “499.

\section{bb) Die Frist}

Die Frist spielt für die Interessenabwägung auch eine große Rolle. Normalerweise hat das Interesse der Veröffentlichung von Information über eine aktuelle Straftat Vorrang vor dem Persönlichkeitsrechtsschutz des Täters. ${ }^{500}$ Aber mit zeitlicher Distanz zur Straftat erhöht sich das Gewicht des Interesses des Täters, um „vor einer Reaktualisierung seiner Verfehlung verschont zu bleiben" ${ }^{\text {501. }}$.

Das bedeutet eben nicht, dass Einzelheiten der Straftat, die nicht mehr aktuell sind, gelöscht werden müssen. „Ein anerkennenswertes Interesse der Öffentlichkeit besteht nicht nur an der Information über das aktuelle Zeitgeschehen, sondern auch an der Möglichkeit, vergangene zeitgeschichtliche Ereignisse zu recherchieren“. ${ }^{502}$ Ein generelles Verbot gegen die Bereithaltung der nicht mehr aktualisierten Straftäter identifizierenden Darstellungen im Internet würde

496 BGHZ 143, 199, 207 = NJW 2000, 103.

497 BVerfG, NZV 2006, 521, Rn. 11; vgl. OLG München, NJW-RR 2003, 111.

498 BGH, NJW 2006, 599, 601, Rn. 29.

499 BVerfGE 35, 202 ff. = NJW 1973, 1226 - Lebach-Fall; BVerfG, NJW 2000, 1859.

500 BGH, NJW 2010, 2728, Rn. 16; siehe auch BGH, NJW 2006, 599, 600, Rn. 14.

501 BGH, NJW 2010, 2728, Rn. 17.

502 BGH, NJW 2010, 2728, 2730, Rn. 21; vgl. BGH, NJW 2010, 757. 
dazu führen, dass „Geschichte getilgt und der Straftäter vollständig immunisiert würde“. 503

Jedoch hat der Betroffene bezüglich einzelner Situationen immer den Anspruch, die ihm belastenden Inhalte zu löschen, wenn er ein überwiegendes persönliches Interesse daran hat.

Es ist zu erwähnen, dass der Zeitfaktor nicht nur auf die Interessenabwägung über Straftäter sondern auch auf die Person aus dem Ereignis anwendbar ist. Mit dem zeitlichen Wandel wird das Informationsinteresse an einem Ereignis immer schwächer; während das Persönlichkeitsrecht der Betroffenen immer mehr Schutz verdient. ${ }^{504}$

\section{b) Nach chinesischem Recht}

Der Persönlichkeitsrechtsschutz des Straftäters wird in China nur gering diskutiert, weil eine Verletzung des Persönlichkeitsrechts des Straftäters in China häufiger nach der Straftat und vor dem Urteil geschieht. Die Menschenfleischsuche hat auch häufig den Verdächtigten als Zielperson gehabt.

Es ist vorstellbar, dass die oben diskutierten Grundprinzipien nach deutschem Recht auch in China eine vergleichbare Anwendung finden.

\section{Die Interessenabwägung im Fall der Verletzung des Persönlichkeitsrechts der Parteien des Gerichtsprozesses}

a) Nach deutschem Recht

Aufgrund $₫ 169$ GVG sind die mündliche Verhandlung sowie die Urteilsverkündung öffentlich. Die Öffentlichkeit der Gerichtsverhandlung rechtfertigt aber nicht automatisch die Veröffentlichung der persönlichen Informationen der beteiligten Parteien, ${ }^{505}$ weil der Grund für die Öffentlichkeit der Gerichtsverhandlung nicht darin liegt, dass alle dazu gehörigen Informationen frei verfügt werden können, sondern an dem Ausschluss von Geheimverfahren und der Kontrolle der Richter und des justizförmigen Verfahrens durch das Volk liegt ${ }^{506}$.

Die Beteiligung am Prozess bedeutet nicht, dass die Parteien eine willkürliche Veröffentlichung ihrer persönlichen Informationen hinnehmen müssen. ${ }^{507}$

503 BGH, NJW 2010, 2728, 2730, Rn. 21; vgl. BGH, NJW 2010, 757.

504 Härting, CR 2009, 21, 24.

505 KG Berlin, K\&R 2007, 535.

506 KG Berlin, K\&R 2007, 535.

507 KG Berlin, K\&R 2007, 535. 
Um einen Eingriff des Persönlichkeitsrechts der Partei zu rechtfertigen, z.B. eine Veröffentlichung des Urteils im Internet unter voller Namensnennung der Parteien, muss hinreichendes, für die Öffentlichkeit erhebliches Informationensinteresse bestehen. ${ }^{508}$ Dies wird wiederum in jedem einzelnen Fall unter Berücksichtigung der betroffenen Rechtsgüter entschieden. ${ }^{509}$ Stellt das Urteil nur den Konflikt der Parteien untereinander dar, wäre beispielsweise eine Veröffentlichung des Urteils mit voller Namensnennung der Parteien ein Verstoß gegen das Persönlichkeitsrecht. ${ }^{510}$

\section{b) Nach chinesischem Recht}

Über die Rechtmäßigkeit der Veröffentlichung persönlicher Informationen der Parteien im Gerichtsprozess in China gibt es in der Entscheidungspraxis, in der Rechtsprechung des Obersten Volksgerichtshofs und in der Literatur unterschiedliche Meinungen.

\section{aa) Die Entwicklung der Meinungen in der Entscheidungspraxis}

Zwischen 2001 und 2008 gab es in China drei ähnliche Klagen in drei verschiedenen regionalen Gerichten gegen die Veröffentlichung des Urteils mit Namensnennung der Partei. Die Verfahren betrafen Rechtsstreitigkeiten zwischen den Hausverwaltungen und den Bewohnern, die wegen irgendwelches Grunds die Hausverwaltungsgebühren nicht bezahlt haben sollten. In den Fällen hatten die Hausverwaltungen ohne Einwilligung der Bewohner den Volltext der gerichtlichen Entscheidungen innerhalb der Nachbarschaft veröffentlicht, um die anderen Bewohner zu warnen, dass jeder die Hausverwaltungsgebühren rechtzeitig bezahlen muss.

Im ersten Fall im Jahr 2002 hatte der Bewohner geklagt, dass das Verhalten der Hausverwaltung sein Recht auf Privatsphäre verletze, weil die in der gerichtlichen Entscheidung beinhaltenden persönlichen Informationen wie Name, Alter, Arbeitgeber veröffentlicht worden sind. Die erste und die zweite Instanz haben entschieden, dass die persönlichen Informationen wie Name, Alter, Arbeitgeber i.V.m. dem Sachverhalt nicht zur Privatsphäre gehören, und haben deswegen die Ansprüche der Bewohner zurückgewiesen. ${ }^{511}$

508 Vgl. OLG Hamburg, ZUM 200866.

509 KG Berlin, K\&R 2007, 535.

510 Vgl. OLG Hamburg, ZUM 200866.

511 Lu Donghong, Southern Metropolis Daily, 1.10.2002. 
Im zweiten Fall im Jahr 2005 hatte ein Bewohner gegen die Hausverwaltung geklagt, weil nach seiner Auffassung die Hausverwaltung eine Ehrverletzung begangen und seine Privatsphäre verletzt habe. Nach der Meinung der Gerichte begründete das Verhalten der Hausverwaltung keine Ehrverletzung, weil die veröffentlichten Informationen wahr waren. Die Verletzung des Rechts auf Privatsphäre war auch nicht begründet, weil kein Verletzungsergebnis entstanden war. Aber das Gericht hat in der Entscheidung festgelegt, dass das Verhalten der Hausverwaltung ungeeignet war. ${ }^{512}$

Im dritten Fall im Jahr 2008 hatte die Hausverwaltung bei der Ausstellung der gerichtlichen Entscheidung die persönlichen Informationen des Bewohners geschwärzt. Der Bewohner behauptete aber, dass die Nachbarn durch den Kontext der Entscheidung sofort erkennen konnten, dass es sich bei der Entscheidung um ihn handelt. Das Gericht hat die Hausverwaltung angewiesen, das Aushängen der gerichtlichen Entscheidung zu beenden. ${ }^{513}$

Die oben genannten drei Entscheidungen sind über die Rechtsstreite, die nicht im Internet passiert sind. Es gab im Jahr 2008 eine Klage wegen Verletzung des Rechts auf Privatsphäre gegen einen Rechtsanwalt, der ein von ihm erfolgreich erstrittenes Urteil mit Namensnennung der Parteien ins Internet gestellt hatte, um für sich zu werben. Nach der Meinung des Gerichts war das Verhalten des Rechtsanwalts fehlerhaft; er wurde als schuldig verurteilt. ${ }^{514}$

Aus den vier Entscheidungen kann man sehen, dass die Gerichte ihre Einstellung über die Veröffentlichung der gerichtlichen Entscheidungen mit Namennennung der Parteien geändert haben. Von „keine Rechtsverletzung“ am Anfang bis zu „ungeeignet“, Unterlassen, bis zum Schluss „fehlerhaft“ und „schuld“ waren die Gerichte mit dem Persönlichkeitsrechtsschutz immer vorsichtiger. ${ }^{515}$

\section{bb) Die Entwicklung der Rechtsprechungen des Obersten Volksgerichtshofs}

Der Oberste Volksgerichtshof hat zu der Frage, ob, wie und zu welchem Ziel die gerichtlichen Entscheidungen veröffentlicht werden sollen, unterschiedliche Auffassungen vertreten.

512 Yang Jianlin, Nan guo jin bao, 10.8.2005, http://www.zhengyanli.org/newshow. asp?id=1573 (besucht am 04.04.2015)

513 Chen Xinglan, Chutian Metropolis Daily 30.12.2008, S. 8, http://www.cnhubei.com/ ctdsb/ctdsbsgk/ctdsb08/200812/t540763.shtml (besucht am 04.04.2015).

$514 \mathrm{Xu}$ Yuxiang/Pan Ying, Jiangsu Legal News, 21.1.2010, S. 1, http://jsfzb.xhby.net/ html/2010-01/21/content_96900.htm (besucht am 04.04.2015).

515 Li Yougen, Legal Science Monthly 2010, No. 5, 126, 128. 
Im Jahr 2000 hat der Oberste Volksgerichtshof eine „Verwaltungsmethode der Veröffentlichung des Urteils oder anderer gerichtlichen Entscheidungen“ erlassen. Aufgrund dieser Methode wurden Urteile über bestimmte Themen ab dem Jahr 2000 veröffentlicht. Ein wichtiger Grund dafür war, die Gerechtigkeit im Gerichtsverfahren zu bewahren.

Im Januar 2007 hat der Oberste Volksgerichtshof „Einige Vorschläge über das Anbieten der Judikative-Unterstützung für die Aufbau harmonischer sozialistischen Gesellschaft" erlassen. Darin wird betont, dass die Urteile veröffentlicht werden sollen. Das Ziel dieser Vorschläge war, die Transparenz der Gerichtsverfahren zu verbessern.

Im Juni 2007 hat der Oberste Volksgerichtshof „Einige Vorschläge zur Verstärkung der Arbeit über das öffentliche Urteil des Gerichts“ erlassen. In den Vorschlägen wird betont, dass die Oberen Volksgerichte Maßnahmen vornehmen sollen, um in ihren zuständigen Bereichen die Veröffentlichung der gültigen Urteile durch Publikation, Internet, LAN zu fördern.

Im Jahr 2009 hat der Oberste Volksgerichtshof „Sechs Regelungen über die Öffentlichkeit der Judikative" erlassen. In diesen Regelungen wurde die Verbreitung der Öffentlichkeit der Judikativen verlangt, um das Auskunftsrecht, das Recht auf Teilnahme, das Recht zu kritisieren und das Aufsichtsrecht der Bürger zu sichern. Zum Ziel des Popularisierens des Rechts, der wissenschaftlichen Forschung, der Anleitung zu den nachträglichen Urteilen, der Standardisierung der Urteile verschiedener Gerichte können Entscheidungen und Urteile gemäß diesen sechs Regelungen publiziert bzw. veröffentlicht werden.

Übrigens haben die sechs Regelungen eine besondere Regel für die online Veröffentlichung der Urteile festgelegt. Die Urteile und gerichtlichen Entscheidungen können im Internet veröffentlicht werden, solange sie nicht einem Staatgeheimnis oder der Privatsphäre unterliegen, oder von einem Minderjährigen begangenes Verbrechen zum Inhalt haben, oder auch mit einer Schlichtung abgeschlossen worden sind und nicht ungeeignet zur Veröffentlichung sind - es sei denn, dass eine der Parteien einen Anspruch auf Veröffentlichung hat. Das Gericht kann zum Schutz der im Urteil genannten Personen notwendige technische Änderung an ihren persönlichen Informationen (wie z.B. Anonymisierung) vornehmen. Eine technische Änderung hier war nach den sechs Regelungen ein „können“ nicht ein „sollen“.

Jedoch hat die „Antwort des Vorschlags über die Löschung der ausführlichen Informationen der Parteien währen der Veröffentlichung der Urteile im Internet" vom Obersten Volksgerichtshof schon im Jahr 2011 festgestellt, dass die Informationen der Parteien und anderer Teilnehmer wie z.B. Anschrift, Kontaktdaten, ID-Nummer, Bankkontonummer durch technische Maßnahmen geändert werden „sollen“. 
Bis hierhin ist es erst offiziell festgelegt, dass die von den Gerichten veröffentlichten Urteile und gerichtlichen Entscheidungen anonymisiert werden müssen.

\section{cc) Die Meinungen in der Literatur über die Anonymisierung der zu veröffentlichen Urteile}

Über die Anonymisierung der zu veröffentlichen Urteile gab es in der Literatur gegensätzliche Meinungen. Dagegen war die Meinung wegen der Öffentlichkeit der Gerichtshandlungen, die ein grundsätzliches Prinzip des Gerichtsverfahrens in China ist. ${ }^{516}$ Dafür war die Meinung unter Berücksichtigung des Persönlichkeitsrechtsschutzes der Parteien. ${ }^{517}$ Es gab auch eine Meinung der Mitte, dass die Anonymisierung darauf ankommt, ob die Veröffentlichung der persönlichen Informationen der Parteien nötig ist, um den Leser zu überzeugen, dass das Urteil gerecht ist. ${ }^{518}$ Die ID-Nummer, die Anschrift der Parteien sind nach dieser Meinung normalerweise zur Veröffentlichung unnötig. Aber das Geschlecht, das Alter, der Arbeitgeber, der Beruf usw. sollen unter Umständen berücksichtigt werden. ${ }^{519}$

Meiner Meinung nach sollen die persönlichen Informationen der Parteien bei der Weiterleitung anonymisiert werden. Es stimmt, dass die Öffentlichkeit der Gerichtshandlungen die Kenntnisnahme der Informationen der Parteien ermöglicht. Aber der Grund der Öffentlichkeit der Gerichtshandlungen ist, die Gerechtigkeit und Transparenz im Gerichtsverfahren zu bewahren. Die Adressaten dieses Prinzips sind die Gerichte und das Gerichtsverfahren, nicht die Parteien.

Übrigens ist die Teilnahme an der Gerichtsverhandlung zeitlich und räumlich begrenzt. Die Menge der Teilnehmer ist begrenzt. Deswegen ist die Kenntnisnahme der Informationen der Parteien bei der Gerichthandlung auch stark begrenzt. Aber durch die Veröffentlichung der Urteile (besonders im Internet) würden die Informationen unbeschränkt weitergeleitet, falls sie nicht anonymisiert würden. Es ist unverhältnismäßig, dass die Parteien wegen eines vielleicht unvermeidlichen Rechtsstreites ihre persönlichen Informationen weltweit verbreiten müssen.

Jedoch soll die Veröffentlichung bestimmter persönlichen Informationen zur Erfüllung des Bedarfs der Öffentlichkeit der Gerichtshandlungen erlaubt werden, wenn sie nötig sind, um den Leser zu überzeugen, dass das Urteil gerecht ist.

516 Li Yougen, Legal Science Monthly 2010, No. 5, 126, 132.

517 Zhao Qi, Legal Forum 2012, No. 6, 115, $118 f$.

518 Li Yougen, Legal Science Monthly 2010, No. 5, 126, 132.

519 Li Yougen, Legal Science Monthly 2010, No. 5, 126, 132. 


\section{$\$ 9$ Die von dem ICP begangenen Rechtsverletzungen}

Eine Rechtsverletzung des ICP kommt in Frage, wenn rechtsverletzender Inhalt auf der von ihm betriebenen Webseite erscheint. Als unmittelbarer Rechtsverletzer hat der ICP bei den Rechtsverletzungen während der Menschenfleischsuche ähnliche Problemen wie der Internetnutzer. Wenn der ICP die persönlichen Informationen der Zielperson zum ersten Mal ins Internet stellt, wird er ähnlich wie der Informationsberichter behandelt. Wenn der ICP die im Internet schon bestehende persönlichen Informationen der Zielperson sammelt und zusammenstellt, wird er ähnlich wie der Informationssammler behandelt. Wenn der ICP selbst die Menschenfleischsuche eingeleitet hat, wird er ähnlich wie der Veranlasser behandelt. Wenn der ICP die Zielperson kommentiert, wird er ähnlich wie der Kommentator behandelt.

Außer den Ähnlichkeiten mit der unmittelbaren Rechtsverletzung von den Internetnutzern gibt es für die vom ICP begangenen Rechtsverletzungen doch einige Besonderheiten, die im Folgenden diskutiert werden.

\section{Nach deutschem Recht}

Um die Rechtsverletzung des ICP zu diskutieren, soll erstmals ein Unterschied zwischen dem ICP und ISP hervorgehoben werden. Ein hier benötigter wesentlicher Unterscheid zwischen dem ICP und dem ISP liegt darin, dass der auf der Webseite vom ICP erschienene Inhalt entweder von dem ICP selbst erstellt oder von seinen Internetnutzern eingetragen aber von ihm zu Eigen gemacht wurde ${ }^{520}$ während der auf der Webseite vom ISP erschienene Inhalt ausschließlich von seinen Internetnutzern eingetragen wurde. Die Beurteilung des Zueigenmachens ist ein wichtiger Schritt für die Diskussion der Providerhaftung, weil der ICP und der ISP dadurch unterschieden werden, während ein ICP und ein ISP bei der Rechtsverletzung völlig unterschiedlich haften. ${ }^{521}$

Für den Fall des selbst geschaffenen Inhalts vom ICP sind zwei Fragen zu diskutieren. Die erste betrifft die Verantwortlichkeit des ICP, wenn der bezügliche Inhalt aus allgemein zugänglichen Quellen kommt. Die zweite Frage betrifft die

520 BGH, CR 2010, 468.

521 Vgl. BGH, NJW 2012, 2345. 
Haftung des ICP, wenn er gleichzeitig als elektronische Presse im Internet darstellt, die das Medienprivileg besetzen könnte.

\section{Zueigenmachen fremder Informationen}

Die Regelungen über die Haftung des ICP folgt aus den $\$ \$ 7$ ff, TMG. Gemäß $₫ 7$ Abs. 1 ist der ICP für seine eigenen Informationen, die er zur Nutzung bereithält, nach den allgemeinen Gesetzen verantwortlich. Fremden Informationen bezüglich findet man die Regelungen in $\$ \$ 8-10$ TMG, die die Übermittelung, Zwischenspeicherung zur beschleunigten Übermittlung und Speicherung von fremden Informationen regeln. Das Verhalten des ICP während der Menschenfleischsuche bezieht sich hauptsächlich auf die Übermittelung der Informationen an die Internetnutzer. ${ }^{522}$ Deswegen ist $\$ 8 \mathrm{TMG}$ anwendbar.

Gemäß $\$ 8$ Abs. 1 TMG sind ICP für fremde Informationen, die er in seinem Kommunikationsnetz übermittelt oder zu denen er den Zugang zur Nutzung vermittelt, nicht verantwortlich, sofern er erstens die Übermittlung nicht veranlasst, zweitens den Adressaten der übermittelten Informationen nicht ausgewählt und drittens die übermittelten Informationen nicht ausgewählt oder verändert hat. Dies setzt gemäß $\$ 8$ Abs. 2 TMG voraus, dass der ICP nicht absichtlich mit seinem Internetnutzer zusammen gearbeitet hat, um rechtswidrige Handlungen zu begehen.

$\$ 8$ TMG ist eine der Haftungsprivilegierungen des ICP für fremden Inhalt. Das bedeutet aber nicht, dass alle Inhalte, die nicht von dem Provider erstellt worden sind, per se von den Haftungsprivilegierungen erfasst sind. Der fremde Inhalt wird als seine eigene Information angesehen, wenn der Provider sich den Inhalt zu Eigen gemacht hat ${ }^{523}$.

Ob ein Provider durch sein Verhalten bestimmten Inhalt zu Eigen gemacht hat, soll aus einer objektiven Sicht auf der Grundlage einer Gesamtbetrachtung aller relevanten Umstände beantwortet werden. ${ }^{524}$ Dieses Prinzip ist vom BGH in seiner "Marions-Kochbuch.de-Entscheidung" entwickelt und vom EuGH bestätigt bzw. als „Neutralitätsgebot-Prinzip“ in seiner Rechtsprechung festgestellt $^{525}$. Das Neutralitätsgebot-Prinzip lautet: Die Haftungsprivilegierungen von fremden Inhalt wurden nicht angewendet, wenn der Provider ,anstatt rein technischer und automatischer Verarbeitung der von seinen Kunden eingegebenen

522 Siehe oben unter $\$ 4$ I 2 c) ff) und gg).

523 BGH, CR 2010, 468.

524 BGH, CR 2010, 468; Nieland, NJW 2010, 1494, 1496.

525 Rössel, CR 2011, 589, 591; BGH, CR 2010, 468, Rn. 22. 
Daten neutral zu erbringen, eine aktive Rolle spielt, die ihm eine Kenntnis dieser Daten oder eine Kontrolle über sie verschaffen konnte“.526

Außerdem hat der BGH zur Beurteilung des Zueigenmachens zahlreiche Prinzipien durch seine Rechtsprechungen entwickelt. Ein Zueigenmachen liegt z.B. regelmäßig vor, wenn die fremde Äußerung so in den eigenen Gedankengang eingefügt wird, dass die gesamte Äußerung als eigene erscheint. ${ }^{527}$ Automatisierte neutrale Prozesse führen prinzipiell nicht zum Zueigenmachen. ${ }^{528}$ Im Gegensatz dazu ist der Inhalt zu Eigen gemacht, wenn er keineswegs ohne inhaltliche Kontrolle automatisch freigeschaltet wurde ${ }^{529}$. Die vor Freischaltungen geführten redaktionellen Kontrollen wie z. B. Überprüfen auf Vollständigkeit und Richtigkeit wird als Zueigenmachen anerkannt. ${ }^{530}$ Aber wenn die fremden Informationen auf der Webseite des ICP extra als fremd gekennzeichnet sind, zählen sie nicht zu den zu Eigen gemachten Informationen, obwohl die Kennzeichnung als eine Art Bearbeitung behandelt werden könnte. ${ }^{531}$

Das gleiche Prinzip hat das Landesgericht Hamburg in einer Entscheidung am 27.4.2007 befolgt. ${ }^{532}$ In dieser Entscheidung wurden die Beiträge der Internetnutzer gleichsam als eigene Informationen des Providers zugerechnet, weil der Provider seinen eigenen Internetauftritt den Internetnutzern zur Verbreitung ihre Beiträge zur Verfügung gestellt hat, während er keine Distanzierung zwischen seinen eigenen Informationen und den fremden Informationen gemacht hat. ${ }^{533}$ Nach der Entscheidung muss der Provider auf seiner Webseite die Inhalte mit unterschiedlichen Quellen nicht pauschal, sondern konkret und ausdrücklich trennen, um zu vermeiden, dass fremder Inhalt als sein eigener Inhalt angesehen wird. ${ }^{534}$

Wäre der fremde Inhalt als zu Eigen gemacht angesehen, muss der ICP für den Inhalt als eigenen Inhalt verantwortlich sein und für die daraus entstehenden Rechtsverletzungen nach den allgemeinen Vorschriften als unmittelbarer Rechtsverletzer haften. ${ }^{535}$

526 Rössel, CR 2011, 589, 591; EuGH, CR 2011, 597.

527 BGH, NJW 2012, 2345, Rn. 11.

528 Rössel, CR 2011, 589, 592.

529 BGH, CR 2010, 468.

530 BGH, CR 2010, 468.

531 Vgl. BGH, NJW 2012, 2345, Rn. 13.

532 LG Hamburg, MMR 2007, 450.

533 LG Hamburg, MMR 2007, 450, 451.

534 LG Hamburg, MMR 2007, 450, 451.

535 BGH, CR 2010, 468. 
Es ist vorzustellen, dass die Diskussion über die von den Internetnutzern begangene Rechtsverletzung auch auf die Rechtsverletzungen des ICP gilt, wenn es um die nach den allgemeinen Vorschriften zu haftende Rechtsverletzung geht. Der Grund liegt darin, dass der ICP und der Internetnutzer beide unmittelbare Verletzer und Adressaten der allgemeinen Vorschriften sind. Deswegen werden im Folgenden nur die Besonderheiten über die vom ICP begangenen Rechtsverletzungen diskutiert.

Der Unterschied zwischen dem ICP und dem Internetnutzer als unmittelbarer Verletzer liegt hauptsächlich darin, dass das BDSG auf der vom ICP begangenen Rechtsverletzung anwendbar ist. Die Besonderheiten über die vom ICP begangenen Rechtsverletzungen stammen deswegen meistens aus den Regelungen des BDSG.

\section{Die Daten aus allgemein zugänglichen Quellen}

Für die Situation, in der sich der ICP als ein Informationssammler verhält, nämlich die schon im Internet veröffentlichte Informationen der Zielperson sammelt, sortiert, analysiert, zusammenfasst und das dadurch entstehende Ergebnis auf seiner Webseite einträgt, ist $\$ 29$ BDSG anwendbar. Gemäß $\$ 29$ Abs. 1 und 2 BDSG ist das geschäftsmäßige Erheben, Speichern, Verändern oder Nutzen personenbezogener Daten zum Zweck der Übermittlung zulässig, wenn die Daten aus allgemein zugänglichen Quellen entnommen werden können oder die verantwortliche Stelle sie veröffentlichen dürfte, es sei denn, dass das schutzwürdige Interesse des Betroffenen an dem Ausschluss der Erhebung, Speicherung oder Veränderung offensichtlich überwiegt. Diese Regelung ist nach der herrschenden Meinung eine Darstellung der Informationsfreiheit i.S.v. Art. 5 Abs. 1 Satz 1 GG. ${ }^{536}$

$\mathrm{Zu}$ den allgemein zugänglichen Quellen zählen die Daten, die mit der $\mathrm{Zu}$ stimmung der Zielperson auf einer im Internet frei zugänglichen Webseite eingestellt werden. ${ }^{537}$ Dementsprechend sollen die Daten aus anderen allgemein zugänglichen Quellen auch in zulässiger Weise ins Internet eingestellt werden. ${ }^{538}$ Sonst besteht die Gefahr, dass der ICP oder andere Informationsberichter die

536 Gola/Schomerus in Gola/Schomerus, BDSG $\$ 28$, Rn. 31; siehe auch Spindler/Nink in Spindler/Schuster, BDSG $\$ 28, \mathrm{Rn} .7$.

537 Seidel/Nink, CR 2009, 666, 669; Peifer/Kamp, ZUM 2009, 185, 187; Oberwetter, BB 2008, 1562, 1564; Ballhausen/Roggenkamp, K\&R 2008, 403, 408; Moos, MMR 2006, $718,719$.

538 Spindler/Nink in Spindler/Schuster, BDSG $\$ 28$, Rn. 7; Weichert, DuD 2009, 7, 11 f. 
Daten erst auf rechtswidrige Weise irgendwo ins Internet stellt, um die spätere Erhebung aus allgemeinen zugänglichen Quellen zu rechtfertigen. ${ }^{539}$

Um den Urstand der Daten zu beachten ist eine Prüfung in jedem einzelnen Fall notwendig. Jedoch soll dies nicht zu einer intensiven Einzelfallprüfung führen, weil eine Interessenabwägung nach $₫ 29$ Abs. 2 BDSG erst nötig ist, wenn das schutzwürdige Interesse der Zielperson offensichtlich überwiegt. Die Offensichtlichkeit soll nach dem Standard eines verständigen Beobachters beurteilt werden. ${ }^{540}$

Trotz der Anwendung von BDSG wird das Verhalten des ICP als Informationssammler nicht anderes als ein normaler Informationssammler reguliert.

\section{Das Medienprivileg}

Die Presse im Internet zählt auch zu dem ICP, weil sie die Beiträge im Internet selbst editiert und offengelegt hat. Beteiligt sie sich an der Menschenfleischsuche, ist ihr Medienprivileg gemäß $₫ 41$ BDSG zu beachten. Das in $₫ 41$ BDSG angeordnete Medienprivileg ist eine Ausprägung der in Art. 5 I 2 GG verankerten Pressefreiheit. ${ }^{541}$ Der Grund des Privilegs liegt darin, dass die journalistische Arbeit unmöglich wäre, wenn die Presse für die Erhebung, Verarbeitung oder Nutzung personenbezogener Daten jedes Mal die Einwilligung der jeweils Betroffenen erhalten muss. ${ }^{542}$

Um das Medienprivileg zu genießen, müssen noch zwei Voraussetzungen erfüllt werden.

\section{a) Rechtmäßiges Subjekt}

Das Medienprivileg des $₫ 41$ BDSG gilt für die „elektronische Presse ${ }^{\text {“543 }}$. Privilegiert sind im Bereich des Internets Online-Verlage mit redaktionellen Strukturen. ${ }^{544}$ „Presseabteilungen von Unternehmen, Verbänden, Parteien und anderen Organisationen, die Onlineausgaben produzieren, fallen nur dann unter den Schutzbereich, wenn sie von der übrigen Struktur eine abgetrennte Organisationseinheit bilden“. ${ }^{545}$

539 Spindler/Nink in Spindler/Schuster, BDSG $\$ 28$, Rn. 7; Weichert, DuD 2009, 7, 11 f.

540 Gola/Schomerus in Gola/Schomerus, BDSG $\$ 28$, Rn. 31.

541 BGH, NJW 2010, 2728, 2731, Rn. 24.

542 BGH, NJW 2010, 2728, 2731, Rn. 24.

543 Herb in Hahn/Vesting, \$ 57 RStV, Rn. 10; BGH, NJW 2009, 2888, 2890.

544 Weichert, VuR 2009, 323, 324.

545 Weichert, VuR 2009, 323, 324. 
Typische Online-Verlage sind die Zeitungs-, Zeitschriften-, Buch- und Fachverlage mit Online-Publikation. ${ }^{546}$ Notwendig ist noch die Anstellung von professionellen Journalisten und anderen Fachleuten, die eine journalistische Redaktion durchführen können. Das bloße Auflisten von Beiträgen unterfällt nicht dem Medienprivileg nach $₫ 41$ BDSG. ${ }^{547}$

\section{b) Zielsetzung der Datenbearbeitung}

Aufgrund $₫ 41$ Abs. 1 muss die Erhebung, Verarbeitung und Nutzung personenbezogener Daten von der Presse ausschließlich zu eigenen journalistischredaktionellen oder literarischen Zwecken erfolgen. ${ }^{548}$ Die Absicht einer Berichterstattung, die Aufgaben einer funktional verstandenen Press zu erfüllen ${ }^{549}$, spielt hier eine entscheidende Rolle. ${ }^{550}$ Die Absicht besteht, wenn sich das Ziel der journalistischen Redaktion auf eine Veröffentlichung der Berichterstattung für einen unbestimmten Personenkreis richtet. ${ }^{551}$

Wenn die zwei Voraussetzungen erfüllt sind, fällt die Tätigkeit des ICP während der Menschenfleischsuche unter das Medienprivileg. Für seine Datenbearbeitung gilt dann nicht mehr das ganze BDSG, sondern nur $\$ 5$ (Datengeheimnis), $\$ 7$ (Schadensersatz), $\$ 9$ (Datensicherheit) und $₫ 38$ a (Verhaltensregeln). ${ }^{552}$ Die Schadensersatzregelung in $\$ 7$ BDSG wird dem entsprechend eingeschränkt, weil er nur für Schäden haften wird, die aus Verletzungen von $₫ 5$ oder $\$ 9$ BDSG resultieren, die mit der hier zu diskutierenden Menschenfleischsuche nicht einschlägig sind. Wegen Offenlegung persönlicher Daten ohne jegliche Einwilligung wird keine Rechtsverletzung bzw. Schadensersatzanspruch der Zielperson begründet.

Das Medienprivileg bedeutet eben nicht, dass solche ICP willkürlich personenbezogene Informationen in ihren Berichterstattungen veröffentlichen können. Als Online-Presse müssen sie die entsprechende journalistisch-ethische Grundregel nachvollziehen, wie z.B. der Pressekodex, der seit dem 1. Januar 2009 auch Verwendung für journalistische Beiträge in Onlinemedien findet ${ }^{553}$. Aufgrund

546 Herb in Hahn/Vesting, \$57 RStV, Rn. 10.

547 Herb in Hahn/Vesting, $\$ 57$ RStV, Rn. 13; BGH, NJW 2009, 2888, 2890.

548 Spindler/Nink in Spindler/Schuster, BDSG $\$ 41, \mathrm{Rn} .3$.

549 Mann/Smid in Spindler/Schuster, Siebter Teil, Rn. 137.

550 BGH, NJW 2010, 2728, 2731, Rn. 24.

551 BGH, NJW 2010, 757, 760.

552 Herb in Hahn/Vesting, $\$ 57$ RStV, Rn. 15.

553 http://www.presserat.info/en/inhalt/der-pressekodex/einfuehrung.html (besucht am 04.04.2015). 
des Kodexes muss die Presse bei der Online-Publikation das Privatleben, die Intimsphäre sowie das Recht auf informationelle Selbstbestimmung des Menschen achten.

\section{Nach chinesischem Recht}

Eine Unterscheidung zwischen ICP und ISP bei der Identifizierung der Providerhaftung ist auch in China von dem Gesetzgeber ${ }^{554}$, der Entscheidungspraxis und der Literatur allgemein anerkannt. ${ }^{555}$ Aber mit der Entwicklung des Internets ist es immer schwieriger geworden, ICP und ISP aufgrund ihrer Funktionen klar zu unterscheiden. ${ }^{56}$ Das höhere Volksgericht von der Provinz Zhejiang hat in $₫ 19$ seiner „Einige Vorschläge über die Beurteilung der Fälle über Urheberrechtsverletzung im Internet" diese Tendenz bereits erwähnt. Nach diesen Vorschlägen soll in jedem konkreten Fall unter Berücksichtigung des konkreten Verhaltens des Providers und des von ihm angebotenen Dienstes beurteilt werden, ob er für die auf seiner Webseite entstandene Rechtsverletzung haften soll; es ist nicht zu empfehlen, nur durch die amtlich angemeldete Funktion des Providers sein Verhalten automatisch in einer Kategorie (ICP oder ISP) zu sortieren. ${ }^{557}$

Wie in Deutschland muss der ICP auch in China die von ihm angebotenen Inhalte überprüfen und für die Rechtswidrigkeit dieser Inhalte verantwortlich sein. Die genauen Pflichten des ICP wurden in der „Vorläufigen Regelung über die Verwaltung der Internetkultur" geregelt, die vom chinesischen Ministerium für Kultur am 11. Feb. 2011 erlassen wurde und am 1. Apr. 2011 in Kraft getreten ist. In $₫ 16$ dieser Regelung ist deutlich festgelegt, was für Inhalte ein ICP anbieten darf. $\$ 18$ ist die Vorschrift über die Prüfungspflicht des ICP für die von ihm angebotenen Inhalte. Gemäß dieser Vorschrift soll der ICP ein „System der Selbstüberprüfung“ entwickeln. Dafür sollen spezielle Abteilungen und Fachleute angestellt werden, die für die Verwaltung des Verhaltens der Internetnutzer und die Überprüfung der Inhalte auf der Webseite verantwortlich sind, um die Rechtmäßigkeit der Inhalte auf der Webseite und des Verhaltens seiner Internetnutzer zu garantieren.

$554 \S 4$, „Regelung einiger Fragen über die Gesetzanwendung auf die Beurteilung der Fälle über den zivilrechtlichen Rechtsstreit wegen Verletzung des informationellen Verbreitungsrechts im Internet" vom chinesischen Obersten Volksgerichtshof.

555 Huang Liangyou, Hebei Law Science 2012, No. 10, 75, 81, 83; Zheng Renrong, Public Administration \& Law 2011, No. 12, 108, 112.

556 Kong Xiangjun, People's Judicature (application) 2012, No. 7, 59, 62.

557 Kong Xiangjun, People's Judicature (application) 2012, No. 7, 59, 62. 
„Zueigenmachen“ ist in chinesischem Recht nicht anerkannt. Die Informationen auf der Webseite sind entweder eigene Informationen vom Provider oder fremde Informationen vom Internetnutzer. Ob der Provider für fremde Informationen haften soll, wird im Rahme der Haftung des ISP diskutiert. ${ }^{558}$

Wie gesagt, in China fehlt ein Datenschutzgesetz. Die hier zu diskutierenden Frage bezüglich findet man nur eine Regelung in $\$ 2$ „InformationsschutzEntscheidung", nach dem die Erhebung und Verwendung persönlicher Informationen nicht ohne Einwilligung des Betroffenen durchgeführt werden darf. Über „allgemein zugängliche Quellen“ und „Medienprivileg“ ist die Entscheidung gar nicht erfasst. Deswegen wird auch nicht zwischen der diesbezüglichen Rechtsverletzung des ICP und der Rechtsverletzung des Internetnutzers unterschieden.

Für die auf seiner Webseite entstandene Rechtsverletzung ist der ICP unmittelbar verantwortlich. Der Rechtsinhaber kann entweder durch „Notice-TakeDown-Verfahren“ vom ICP verlangen, Maßnahmen vorzunehmen, um die Rechtsverletzung zu stoppen, oder direkt den ICP vor Gerichte beklagen. ${ }^{559}$

558 Siehe unten unter $\$ 10$ II 2 c) ff).

559 Xu Wei, Modern Law Science 2013, No. 1, 58, 66. 


\section{$\$ 10$ Die von dem ISP begangenen Rechtsverletzungen}

Im Vergleich mit dem ICP bietet der ISP keine eigenen (oder zu Eigen gemachten) Informationen an, sondern nur eine Plattform für die Internetnutzer, auf der sie Beiträge eintragen, speichern und veröffentlichen können. Mit der Entwicklung des „Web 2.0“ sind die Plattforen in enorm unterschiedlichen Arten gewachsen, wie z.B. Foto- und Videoportalen, sozialen Online-Netzwerken und einer Vielzahl von Foren, in denen Meinungen und Informationen ausgetauscht werden $^{560}$. Neben den unterschiedlichen Formen haben die ISP eine Gemeinsamkeit: objektiv gesehen haben sie die von ihren Nutzern eingetragenen Beiträge gespeichert und für den Abruf auch von den anderen Internetnutzern bereitgestellt. ${ }^{561}$

Über die Rechtsverletzung des ISP während der Menschenfleischsuche sind zwei Fragen zu beantworten: soll der ISP wegen der auf seiner Webseite entstandenen Rechtsverletzung als unmittelbarer Verletzer haften; ob und wie soll er für die von seinen Nutzer eingetragenen fremden Informationen auf seiner Webseite als mittelbarer Verletzer haften.

\section{Nach deutschem Recht}

\section{Die Möglichkeit des ISP als unmittelbarer Verletzer zu haften}

Die oben genannten unmittelbaren Rechtsverletzungen der Internetnutzer unterschiedlicher Art können nur mit Benutzung der vom ISP betriebenen Webseite verwirklicht werden. Das heißt, der ISP hat durch das Anbieten seines Dienstes bei den Rechtsverletzungen seiner Nutzer tatsächlich geholfen. Die Begründung einer Rechtsverletzung des ISP setzt ferner voraus, dass der ISP für sein Verhalten Schuld hat. Um die Schuld zu beurteilen, soll die Pflicht des ISP beim Anbieten seines Diensts untersucht werden.

560 Nieland, NJW 2010, 1494.

561 Ehmann in Simitis, BDSG $₫ 29$, Rn. 96; Spindler/Nink in Spindler/Schuster, $₫ 28$ BDSG, Rn. 3; BGH, BGHZ 181, 328, Rn. 23ff. = NJW 2009, 2888, $2891=\mathrm{MMR}$ 2009, 608, 610 . 


\section{a) Der ISP hat keine allgemeine Prüfungspflicht}

$\$ \$ 7$ ff TMG sind auch auf die Situation des ISP anwendbar. Gemäß $₫ 7$ Abs. 2 TMG ist ISP nicht verpflichtet, die von ihm übermittelten oder gespeicherten Informationen zu überwachen oder nach Umständen zu forschen, ob diese auf eine rechtswidrige Tätigkeit hinweisen. Durch diesen Paragraphen ist die allgemeine Prüfungspflicht des ISP auf die von ihm übermittelten Informationen deutlich verneint. Dies hat zur Folge, dass der ISP normalerweise nicht als Täter oder Mittäter haften soll.

\section{b) Der ISP haftet normalerweise nicht als Täter oder Mittäter}

Die Ausschließung der allgemeinen Prüfungspflicht befreit den ISP von der Identifizierung als Täter oder Mittäter. ${ }^{562}$ Der ISP bietet gemäß seinem BetriebsModus seinen Nutzern die Plattformen, auf denen die Nutzer selbstständig Informationen eintragen ${ }^{563}$. Die eingetragenen Informationen werden automatisch gespeichert und abrufbar gemacht. Weil der ISP nicht pflichtig ist, die eingetragenen Informationen zu überprüfen, wird er dann auf keinen Fall Kenntnis über den Inhalt der eingetragenen Informationen haben. Wäre aus diesen Informationen eine Rechtsverletzung gekommen, könnte dem ISP kein bewusstes oder gewolltes Zusammenwirken zugeordnet werden. ${ }^{564}$ Durch den automatisierten Hochladenprozess scheidet die vorsätzliche Teilnahme des ISP an einer möglichen Rechtsverletzung aus. ${ }^{565}$ Für die Begründung einer Täter- oder Mittäterhaftung des ISP fehlt das Bewusstsein der Rechtswidrigkeit. ${ }^{566}$

Es könnte die Ansicht vertreten werden, dass allein die Bereitstellung der Plattform schon den Vorsatz vom Überlassen der in der Zukunft möglich passierenden Rechtsverletzungen begründet, und der ISP deswegen als Gehilfe haften soll. Der BGH hat diese Ansicht in seinem „Internetversteigerung II - Urteil“ für unbegründet gehalten. ${ }^{567}$ Nach der Meinung des BGH muss ein Gehilfe einen bedingten Vorsatz auf eine konkrete Haupttat haben; allein das generelle Bewusstsein, dass überhaupt Rechtsverletzungen stattfinden können, genügt nicht. ${ }^{568}$ Der Hintergedanke liegt darin, dass jeder erlaubte Dienst des Providers

562 Ensthaler/Heinemann, GRUR 2012, 433.

563 Ensthaler/Heinemann, GRUR 2012, 433.

564 Ensthaler/Heinemann, GRUR 2012, 433.

565 BGH, GRUR 2004, 860, 864 - Internetversteigerung I.

566 BGH, GRUR 2004, 860, 863f.

567 BGH, GRUR 2011, 152, 154; GRUR 2007, 708, 710.

568 Spindler, MMR 2007, 507, 513. 
für rechtswidrigen Zweck verwendet werden kann. Eine allgemeine Vorsatzvermutung führt zum Schluss wiederum zu einer allgemeinen Prüfungspflicht des ISP, die vom Gesetz ausdrücklich verboten ist.

Ferner hat der BGH in seiner Rechtsprechung festgelegt, dass lediglich das Verbreiten der fremden Äußerung ohne inhaltliche Änderung auch von Art. 5 Abs. 1 Satz 1 GG geschützt ist ${ }^{569}$. Allein in der Übermittelung fremder Informationen liegt weder eine Täterschaft noch eine Teilnahme an einer Vervielfältigung oder einer öffentlichen Zugänglichmachung, ${ }^{570}$ solange der Provider gemäß $₫ 8$ Abs. 1 TMG die Übermittlung nicht veranlasst, den Adressaten der übermittelten Informationen nicht ausgewählt und die übermittelten Informationen nicht ausgewählt oder verändert hat.

Das bedeutet gleichzeitig, dass, wenn der ISP die Bedingungen von $\$ 8$ Abs. 1 TMG nicht einhält, er dessen Schutzbereich verlassen könnte, und wiederum als Anstifter haftet.

\section{c) Unter bestimmten Umständen haftet der ISP als Anstifter}

Die Internetwelt ist nicht ausschließlich von den Internetnutzern strukturiert. Die Betreiber der Webseiten möchten auch eine Rolle spielen, um ihre angestrebten wirtschaftlichen oder andere Interessen zu erkämpfen. Deswegen sucht der ISP manchmal kontroverse Themen aus, um darüber eine Diskussion auf seiner Webseite zu provozieren. Davon erwartet sich der Provider mehr Aufmerksamkeit und mehr Nutzer, die dem Provider mehr wirtschaftliches Einkommen bringen könnten.

Würde diese Diskussion in Form von Menschenfleischsuche durchgeführt, und wären die persönlichen Informationen der Zielperson von den Internetnutzern rechtswidrig übermittelt worden, hätte der Provider tatsächlich die Übermittelung veranlasst. Deswegen wird er den Schutzbereich von $\$ 8$ TMG verlassen, und muss für die rechtswidrigen fremden Informationen verantwortlich sein.

Unter diese Umstände entsteht für den provozierenden ISP eine haftungsvermeidende Obliegenheit ${ }^{571}$, den Inhalt der Diskussion proaktiv zu überwachen. Sofern und sobald wegen der provozierten Diskussion die Persönlichkeitsrechte des Betroffenen durch einen Dritten tatsächlich verletzt sind, wird der

569 BGH, NJW 2012, 2345, 2346, Rn. 18; vgl. BGH, NJW 2010, 760.

570 Wilmer, NJW 2008, 1845, 1846.

571 Nieland, NJW 2010, 1494, 1499. 
Forenbetreiber als Anstifter anerkannt ${ }^{572}$ und als Mittäter der deliktischen Handlung nach $₫ 830$ II BGB haften. ${ }^{573}$

Meiner Meinung nach kann und soll der ISP unter diesem Umstand als ICP angesehen werden und haften. Gemäß $\$ 8$ Abs. 1 TMG hat der Provider durch die Auswahl kontroverser Themen und die Provozierung einer Diskussion die von den Internetnutzern eingetragenen fremden Informationen zueigengemacht. ${ }^{574}$ Er soll für die Rechtswidrigkeit dieser Informationen verantwortlich sein.

\section{d) Unter bestimmten Umstände haftet der ISP als Gehilfe}

Gemäß $\$ 10$ TMG soll der ISP unverzüglich tätig sein, um die Information zu entfernen oder den Zugang zu ihr zu sperren, sobald er diese Kenntnis erlangt hat. Wenn der ISP die Handlungspflicht bewusst missachtet, kann eine Gehilfenhaftung durch Unterlassen begründet werden. ${ }^{575}$ Die Handlungspflicht aus der Gehilfenhaftung ist mit der aus der Störerhaftung kaum zu unterscheiden. ${ }^{576}$ Deswegen „kreuzen sich hier die Anspruchsvoraussetzungen aus den unechten Unterlassungsdelikten mit den Unterlassungs- und Beseitigungsansprüchen aus der Störerhaftung " ${ }^{577}$ Allerdings sollen Störerhaftung und Gehilfenhaftung streng getrennt werden; sonst besteht die Gefahr, dass jeder Störer automatisch zum Gehilfen würde. ${ }^{578}$

Um mit der Störerhaftung zu unterscheiden, ist zur Begründung einer Gehilfenhaftung ein doppelter Vorsatz erforderlich. ${ }^{579}$ Das heißt, der ISP muss zumindest eine objektive Beihilfenhandlung bedingt vorsätzlich erbringen, und zumindest einen bedingten Vorsatz in Bezug auf die Haupttat haben. ${ }^{580}$ Die Einzelheiten über die Tat muss der ISP nicht genau wissen. ${ }^{581} \mathrm{Im}$ Gegenteil braucht für die Begründung einer Störerhaftung nur ein Verstoß gegen die zumutbare

572 Nieland, NJW 2010, 1494, 1499.

573 Nieland, NJW 2010, 1494, 1499.

574 Siehe oben unter $\$ 9$ I 1.

575 Ensthaler/Heinemann, GRUR 2012, 433, 440.

576 Ensthaler/Heinemann, GRUR 2012, 433, 434.

577 Ensthaler/Heinemann, GRUR 2012, 433, 434.

578 Rempe, MMR 2013, 533, 535.

579 Ensthaler/Heinemann, GRUR 2012, 433, 436.

580 Siehe OLG, Hamburg, MMR 2013, 533, 534; vgl. auch Ensthaler/Heinemann, GRUR 2012, 433, 439; und Heine in Schrönke/Schröder, StGB \$27, Rn. 19.

581 Ensthaler/Heinemann, GRUR 2012, 433, 436. 
Handlungspflicht. ${ }^{582}$ Es ist jedoch häufig schwierig zu beurteilen, ob eine Nichterfüllung der Handlungspflicht vom ISP vorsätzlich gemacht ist.

Das OLG Hamburg hat in seiner Entscheidung am 13.5.2013 klargestellt, dass nicht bei jedem nicht unverzüglichen Tätigwerden gegen die rechtsverletzende Information sogleich eine Haftung als Gehilfe in Betracht kommen wird, vielmehr dürfte ein verzögertes Tätigwerden im Regelfall lediglich eine Störerhaftung begründen, weil es insoweit am Nachweis eines Gehilfenvorsatzes fehlen dürfte. ${ }^{53}$ Aber ein hartnäckiges Ignorieren der Rechte des Betroffenen, wie z.B. in diesem Fall, dass der ISP nach dem Erlangen der Beanstandung durch mehrere E-Mails und sogar anwaltlich Schreiben immer noch keine Maßnahmen vorgenommen hat, begründet schon einen Vorsatz. ${ }^{584}$

Jedoch wollte das OLG Hamburg in dieser Entscheidung keine starre Regel aufstellen, ab welchem Grad der Verzögerung des Tätigwerdens eine Gehilfenhaftung in Betracht kommt. ${ }^{585}$ Ein Vorsatz für einen solchen Fall kann auch nur in jedem einzelnen Fall unter Berücksichtigung der gesamten Konstellation beurteilt werden.

\section{Die Situation, der ISP haftet als Störer}

Obgleich der ISP keine Prüfungspflicht hat und deswegen nicht als Täter für die Rechtsverletzung haftet, liefert er als „Herr des Forums“ zumindest einen technischen Verursachungsbeitrag ${ }^{586}$, der tatsächlich zur Rechtsverletzung führt. ${ }^{587}$ Rechtsdogmatisch kann das Verhalten des ISP über das Instrument der Störerhaftung reguliert werden, das eine Verbreitung der Schuldnergruppe zur Ausweitung des Rechtsschutzes bezweckt. ${ }^{58}$ Dies ist für die vom Internetnutzer begangene Rechtsverletzung besonders sinnvoll, weil der Internetnutzer als unmittelbarer Verletzer wegen Anonymität und Pseudonymität im Internet häufig schlecht verfolgbar bzw. identifizierbar ist, ${ }^{589}$ während der Provider dagegen für die Ansprüche der Betroffenen erreichbar bleibt. ${ }^{590}$

Die Störerhaftung folgt aus $\$ 1004$ BGB, die original auf den Fall der Besitzentziehung richtet. Dieser Anspruch ergänzt den Vindikationsanspruch

582 Ensthaler/Heinemann, GRUR 2012, 433, 439.

583 OLG, Hamburg, MMR 2013, 533, 534.

584 OLG, Hamburg, MMR 2013, 533, 534

585 OLG, Hamburg, MMR 2013, 533, 534

586 Nieland, NJW 2010, 1494.

587 Gräbig, MMR 2011, 504.

588 Gräbig, MMR 2011, 504.

589 Nieland, NJW 2010, 1494.

590 Nieland, NJW 2010, 1494. 
aus $₫ 985$ BGB. ${ }^{591}$ Die Anwendung der Norm bleibt jedoch nicht nur im Bereich des Sachenrechts. Nach der Rechtsprechung und h.M. ist eine Analogie auf den Schutz aller absoluten Rechte sowie alle durch $\$ \$ 823$ Abs. 2, 824 bis 826 BGB deliktisch geschützten Rechte und Rechtsgüter möglich ${ }^{592}$. Dazu gehört offensichtlich auch der Schutz der allgemeinen Persönlichkeitsrechte im Internet. ${ }^{593}$

Die Störerhaftung soll nicht zu einer Gefährdungshaftung ausufern. ${ }^{594} \mathrm{Um}$ die Störerhaftung zu beurteilen, müssen einige verlässliche Grundkriterien verfolgt werden.

\section{a) Unmittelbare Rechtsverletzung durch eine Drittperson}

Als Störer hat der ISP nicht selbst die unmittelbare Rechtsverletzung vorgenommen. Eine von einer Drittperson begangene Rechtsverletzung muss vorliegen. Für die Störerhaftung des ISP während der Menschenfleischsuche muss festgestellt werden, dass das Verhalten des Informationsberichters, Informationssammlers, Veranlassers oder des Kommentators eine Rechtsverletzung des Persönlichkeitsrechts der Zielperson begründet.

\section{b) Die Beurteilung des ISP als Störer}

Störer für eine Rechtsverletzung kann derjenige sein, der - ohne Täter oder Teilnehmer zu sein - in irgendeiner Weise willentlich und adäquat kausal zur Verletzung eines geschützten Gutes beiträgt. ${ }^{595}$ Eine Störerhaftung ist nach der allgemeinen Definition verschuldensunabhängig und unabhängig von der Art und dem Umfang des Tatbeitrags des Störers. ${ }^{596}$ Für die Feststellung eines Störers genügt es, „die Handlung eines eigenverantwortlich handelnden Dritten zu unterstützen oder auszunutzen, und dabei bei der Herbeiführung der rechtswidrigen

591 Spindler/Anton in Spindler/Schuster, $\$ 1004$ BGB, Rn. 1.

592 Spindler/Anton in Spindler/Schuster, \$ 1004 BGB, Rn. 1; BGHZ 14, 163, 170; Gursky in Staudinger, $\$ 1004$ BGB Rn. 15f.; Baldus in Münchener Kommentar zum BGB, $\S 1004$ BGB Rn. 6; Fritzsche in Bamberger/Roth, $\$ 1004$ BGB Rn. 2 ff.; Bassenge in Palandt, $₫ 1004$ BGB Rn. 4.

593 Spindler/Anton in Spindler/Schuster, $\$ 1004$ BGB, Rn. 1.

594 Wilmer, NJW 2008, 1845, 1849.

595 BGH, GRUR 2004, 860, 864; vgl. BGHZ 148, 13, 17; BGH, GRUR 2002, 618, $619=$ WRP 2002, 532.

596 Spindler/Anton in Spindler/Schuster, $\$ 1004$ BGB, Rn. 8; BGHZ 148, 13, 17 = NJW 2001, 3265, 3266; BGH, GRUR 2002, 618, 619; BGHZ 158, 236, 251 = MMR 2004, 668,671 . 
Beeinträchtigung mitzuwirken, sofern der Inanspruchgenommene die technische und rechtliche Möglichkeit zur Verhinderung dieser Handlung hatte ${ }^{\text {(6597. }}$.

Der Provider hat willentlich eine Internetplattform eröffnet, die tatsächlich eine Unterstützung der Handlung des Internetnutzers ist, um die eigenverantwortlichen Informationen des Nutzers auf der Webseite einzutragen. Wenn die eingetragenen Informationen rechtswidrig sind und damit die Rechte des Betroffenen verletzen, hat der Provider bei der Herbeiführung mitgewirkt, weil der Provider die Kompetenz hat, rechtswidrige Beiträge von seiner Webseite zu löschen oder zu sperren, um eine Rechtsverletzung zu verhindern. Dies erfüllt wiederum die oben genannte Voraussetzung von technischer und rechtlicher Möglichkeit. $\mathrm{Ob}$ der Provider eine Prüfungspflicht hat, ob er seine Prüfungspflicht verletzt hat, oder ob er schuld ist, spielt hier keine Rolle. Der Provider ist der Störer der von seinem Nutzer auf seiner Webseite begangener Rechtsverletzung.

\section{c) Zumutbarkeit}

Die Beziehung von Störer und Störerhaftung ist zu verdeutlichen. Der Provider kann Störer sein, muss aber nicht unbedingt als Störer haften. Um eine Störerhaftung zu begründen, muss es dem ISP rechtlich und tatsächlich zumutbare sein, die unmittelbare Rechtsverletzung zu verhindern. ${ }^{598}$ Dazu werden vor allem die Quantität und Qualität der Rechtsverletzungen, ob der Provider bezüglich der rechtswidrigen Inhalte für sich Werbung macht, die Frage des Profitierens an Rechtsverletzungen und die Schaffung alternativer Löschmöglichkeiten für Betroffene berücksichtigt. ${ }^{599}$

Wären die oben genannten Voraussetzungen erfüllt, müsste der ISP als Störer haften. Daraus entstehen die Beseitigungs- und Unterlassungspflicht des ISP, wonach er die bezogenen Inhalte rechtzeitig entfernen oder sperren und unter Umständen Maßnahmen vornehmen muss, um die zukünftigen gleichartigen Rechtsverletzungen zu vermeiden. Wären diese Pflichten nicht erfüllt, müsste der ISP wegen Pflichtverletzung für den daraus entstehenden Schaden haften.

\section{d) Kenntnis}

Der Provider kann von der Störerhaftung i.S.v. $\$ 10$ TMG privilegieren, wenn er keine Kenntnis von der rechtswidrigen Handlung oder der Information hat.

597 Spindler/Anton in Spindler/Schuster, $\$ 1004$ BGB, Rn. 8; BGHZ 148, 13, $17=$ NJW 2001, 3265, 3266; BGH, GRUR 2002, 618, 619; BGH, GRUR 1999, 418, 420 = NJW 1999, 1960, 1960; BGHZ 158, 236, 251 = MMR 2004, 668, 671.

598 Gräbig, MMR 2011, 504, 505.

599 Wilmer, NJW 2008, 1845, 1849. 
Nach der herrschenden Meinung bedeutet Kenntnis hier menschliche Kenntnis in Form von positiver Kenntnis. ${ }^{600}$ Die bloße Möglichkeit oder Wahrscheinlichkeit der Existenz eines rechtswidrigen Inhalts ${ }^{601}$ oder ein „Kennenmüssen“ im Sinne einer groben Fahrlässigkeit ist hier nicht ausreichend. ${ }^{602}$

\section{Nach chinesischem Recht}

\section{Der ISP hat prinzipiell keine allgemeine Prüfungspflicht}

Wie oben gesagt wurde, werden ICP und ISP in China auch deutlich unterschieden. Dementsprechend hat der ICP eine allgemeine Überwachungspflicht. Im Gegensatz dazu werden die allgemeine Überwachungspflicht und die verschuldensunabhängige Haftung des ISP von der Justizpraxis verneint. ${ }^{603}$ Aufgrund $\$ 8$ Abs. 2 „Regelung einiger Fragen über die Gesetzesanwendung auf die Beurteilung der Fälle über den zivilrechtlichen Rechtsstreit wegen Verletzung des informationellen Verbreitungsrechts im Internet ${ }^{\text {“604 }}$ sollen die Gerichte den ISP nicht als schuldig verurteilen, nur weil er das rechtswidrige Verhalten der Internetnutzer nicht aktiv geprüft hat. Durch diese Vorschrift ist die allgemeine Prüfungspflicht des ISP offiziell verneint.

\section{Unter bestimmten Umstände haftet der ISP gesamtschuldnerisch mit seinen Nutzern}

\section{a) Gesetzliche Grundlage}

Die allgemeine Regelung über die Haftung des ISP befindet sich in $\$ 36$ des Delikthaftungsgesetzes: verletzt der Internetnutzer oder der ISP mittels Internet die zivilrechtlichen Rechte oder Interessen der anderen, sollen sie dafür

600 Leible/Sosnitza, WRP 2004, 592, 596; Müller-Broich, TMG \$10, Rn 4; Hoffmann in Spindler/Schuster, TMG $\$ 10, \mathrm{Rn} .18$.

601 Vgl. BGH, GRUR 2007, 890, Rn. 46; und Müller-Broich, TMG \$10, Rn 4.

602 Hoffmann in Spindler/Schuster, TMG $\$ 10$, Rn. 18; Müller-Broich, TMG $\$ 10$, Rn 4; Vgl. OLG München, MMR 2002, 611, 612.

603 Vgl. Peking Zweites Mittleres Volksgericht, Urt. v. 28.11.2000 - (2000) er zhong zhi chu zi di 18 hao; Huang Liangyou, Hebei Law Science 2012, No. 10, 75, 83; Zhou Hua, Journal of China Three Gorges University (Humanities \& Social Sciences) 2012, No. 5, 87; Wu Haijie, Youth Times 11.02.2012, S. A04; siehe auch Yang Qinghui, Chinacourt.org, 20.02.2010, http://old.chinacourt.org/html/article/201001/20/392004.shtml/2011-08-04 (besucht am 04.04.2015).

604 Vom chinesischen Obersten Volksgerichtshof. 
Delikthaftung tragen; wenn ein Internetnutzer mit Benutzung des Internets eine Rechtsverletzung begangen hat, hat der Rechtsverletzte das Recht, den ISP zu informieren und nötige Maßnahmen vom ISP zu verlangen, um den rechtswidrigen Inhalt zu löschen, zu sperren oder das Verlinken zu diesem Inhalt abzubrechen; wenn der ISP nach Erlangen der Informierung nicht rechtzeitig nötige Maßnahmen vornimmt, haftet er mit demjenigen Internetnutzer gesamtschuldnerisch für den erhöhten Schaden; wenn der ISP wusste, dass der Internetnutzer unter Benutzung seines Internetdienstes die zivilrechtlichen Rechte oder Interessen der anderen verletzt und nicht nötige Maßnahmen vorgenommen hat, haftet er mit demjenigen Internetnutzer gesamtschuldnerisch für den gesamten Schaden. Im Fall von Menschenfleischsuche soll dieser Paragraph wie folgt ausgelegt werden:

ISP ist nicht verpflichtet, den Inhalt der Menschenfleischsuche allgemein zu überwachen. Die Pflicht des ISP entsteht ab den Zeitpunkt, wenn er die Rechtsverletzung zur Kenntnis nimmt. Kenntnisnahme könnte vorliegen, wenn die Rechtsverletzung offensichtlich ist oder der Rechtsverletzte den ISP von der Verletzung in Kenntnis gesetzt hat. Ab den Zeitpunkt der Kenntnisnahme entsteht für den ISP die Pflicht, die rechtsverletzenden Inhalte zu entfernen oder zu sperren. Wenn der ISP diese Pflicht nicht rechtmäßig oder rechtzeitig durchführt, haftet er wegen Pflichtverletzung. Die Haftung beschränkt sich auf den durch seine Pflichtverletzung erhöhten Teil des Schadens. Aber wenn der ISP die Rechtsverletzung schon von Anfang an zur Kenntnis genommen hat, oder wenn der Schaden erst ab dem Zeitpunkt seiner Kenntnisnahme entsteht, soll er dann für den gesamten Schaden mit dem unmittelbaren Rechtsverletzer gesamtschuldnerisch haften.

\section{b) Der Grund der gesamtschuldnerischen Haftung des ISP}

Der ISP in China muss für durch seine Pflichtverletzung entstandenen Schaden gemeinsam mit dem unmittelbaren Verletzer gesamtschuldnerisch haften. Über die Grundlage der gesamtschuldnerischen Haftung gibt es in der Literatur unterschiedliche Meinungen.

\section{aa) Gesamtschuldnerische Haftung aufgrund Gehilfenhaftung}

Es herrscht die Meinung, dass der ISP bei der Rechtsverletzung seines Nutzers eine Rolle wie ein Gehilfe spielt. Die gesamtschuldnerische Haftung resultiert aus der Gehilfenhaftung. ${ }^{605}$ Diese Meinung hat ihren Nachteil, weil Gehilfenhaftung

605 Vgl. Wu Handong, Studies in Law and Business 2010, No. 6, 29. 
den Vorsatz zur Voraussetzung hat, was bei der Rechtsverletzung häufig nicht der Fall ist. Der ISP hat keine allgemeine Überwachungspflicht, und die rechtswidrige Information wurde vom Internetnutzer selbstständig eingetragen. Dies führt dazu, dass der ISP die während der Menschenfleischsuche entstandene Rechtsverletzung nicht voraussehen kann. Er kann dementsprechend auch keinen Vorsatz haben, dem Nutzer beim Rechtsverletzen zu helfen, außer der ISP betreibt seine Webseite nur oder hauptsächlich für rechtswidrige Zwecke, was dann den Anwendungskreis dieses Paragraphs im großen Maße einschränkt.

\section{bb) Gesamtschuldnerische Haftung aufgrund Mittäterhaftung}

Nach der herrschenden Meinung in der Literatur haftet der ISP gesamtschuldnerisch mit dem unmittelbaren Rechtsverletzer, weil er aufgrund des DienstAnbietens ein Mittäter ist. ${ }^{606}$ Diese Meinung ist auch in der Praxis und von den Gerichten allgemein anerkannt. ${ }^{607}$

Die Beurteilung eines Mittäters besteht nach $₫ 3$ „Erklärung einiger Fragen über die Gesetzesanwendung für die Beurteilung der Fälle über den aufgrund von personenbezogenen (körperliche und geistliche) Rechtsverletzungen entstehenden Schadenersatzanspruch" vom chinesischen Obersten Volksgerichtshof. Der Gesetztext aus $₫ 3$ dieser Erklärung lautet: wenn mehr als zwei Personen wegen gemeinsamer Absicht oder Fahrlässigkeit zum Schaden der Anderen führen, oder wenn sie keine gemeinsame Absicht oder Fahrlässigkeit haben, aber ihre rechtsverletzenden Verhalten miteinander unmittelbar kombinieren und zusammen zu einem Schaden der Anderen führen, sind sie Mittäter und sollen gemäß $₫ 130$ AGZR gesamtschuldnerisch haften.

Für die Mittäterhaftung ist es nicht nötig zu beweisen, dass es zwischen den Mittätern Absprachen oder eine gemeinsame Schuld besteht. ${ }^{608}$ Auch die gemeinsame Fahrlässigkeit führt zur Mittäterhaftung, solange die Verhalten von den Tätern gemeinsam zum Schaden führen. Entweder vorsätzlich oder fahrlässig, solange der ISP seine Handlungspflicht, die rechtswidrige Information zu entfernen oder sperren, nicht erfüllt, und dadurch eine Rechtsverletzung entsteht, ist er Mittäter.

606 Yang Lixin, Tort Liability Law, S. 112-114; Qian, Yuwen/Zhang, Jialin, Intellectual Property 2013, No. 2, 44, 48; Cheng Yan, Internet Law Review 2012, No. 1, 201, 208; Chen Jinchuan, Journal of Law Application 2011, No. 6, 52, 54.

607 Vgl. Peking Oberes Volksgericht, Urt. v. 2.12.2004 - (2004) gao min zhong zi di 1303 hao; Peking Oberes Volksgericht, Urt. v. 20.12.2007 - (2007) gao min zhong zi di 1188 hao; siehe auch Chen Jinchuan, Journal of Law Application 2011, No. 6, 52, 54-55.

608 Xue Hong, China Copyright 2011, No. 5, 36, 37. 


\section{cc) Gesamtschuldnerische Haftung wegen öffentlicher Politik}

Es herrscht auch die Meinung, dass die gesamtschuldnerische Haftung des ISP ein Abwägungsergebnis unter Berücksichtigung der öffentlichen Politik ist. ${ }^{609}$ Diese Meinung ist auch überzeugend.

Nach der herrschenden Meinung basiert die Identifizierung des ISP als Mittäter darauf, dass er die Pflicht verletzt hat, die rechtswidrigen Informationen rechtzeitig zu sperren oder zu entfernen. Der Urgrund dieser Pflicht liegt darin, dass der normale Internetnutzer als der unmittelbare Rechtsverletzer wegen der Anonymität und Pseudonymität für den Rechtsverletzten schwierig zu identifizieren ist, während der ISP als Meister des Internets tatsächlich die Kompetenz hat, die Erweiterung der Rechtsverletzung zu vermeiden. Um die Rechte der Rechtsverletzten so schnell wie möglich zu schützen, soll diese Handlungspflicht unter Berücksichtigung der öffentlichen Politik beim ISP liegen. ${ }^{610}$

Es ist vorstellbar, wenn die Entwicklung der Technik in der Zukunft neue Methoden hervorbringt, dass der Rechtsverletzte die Rechtsverletzung ohne Hilfe des ISP selbst stoppen könnte; die Handlungspflicht des ISP wäre dann nicht mehr erforderlich. Unter Berücksichtigung der öffentlichen Politik jener Zeit entfiele dann eine gesamtschuldnerische Haftung.

\section{dd) Gesamtschuldnerische Haftung wegen Gefährdungshaftung}

Es gibt noch die Meinung die gesamtschuldnerische Haftung durch die Theorie der Gefährdungshaftung auszulegen. Nach dieser Meinung ist der Kommunikationsraum im Internet genauso wie der öffentliche Raum im realen Leben, in dem nicht nur die Gefahr der Verletzung von Persönlichkeitsrechten und des geistigen Eigentums, sondern auch der potenzielle Ansatz besteht, der zu Körperverletzung und Vermögensverletzung im normalen Leben führt. ${ }^{611}$ Darum ist die Situation über die Pflicht der Sicherheitsgarantie von den Verwaltungen öffentlicher Plätze wie Hotel, Kaufhaus, Bank, Bahnhof, Vergnügungseinrichtung oder von den Veranstaltern öffentlicher Aktivitäten i.S.v. $\$ 37$ Delikthaftungsgesetz anwendbar. ${ }^{612}$ Der ISP hat durch das Anbieten des Internetdiensts die potenziell gefährliche Situation geschaffen, dass auf seiner Webseite eine Rechtsverletzung entstehen könnte. Deswegen wird es die Pflicht des ISP sein,

609 Yang Lixin, Journal of National Prosecutors College 2010, No. 2, 3, 9.

610 Cai Chang, Studies in Law and Business 2013, No. 2, 113, 115.

611 Liu Wenjie, Peking University Law Journal 2012, No. 2, 395, 396.

612 Liu Wenjie, Peking University Law Journal 2012, No. 2, 395, 396. 
die Sicherheit auf seiner Webseite zu garantieren. Eine Verletzung dieser Pflicht führt zur Gefährdungshaftung. ${ }^{613}$

Jedoch gibt es auch eine Gegenmeinung, die meint, dass die traditionelle Pflicht der Sicherheitsgarantie im öffentlichen Raum anders als die Pflicht des ISP ist. Der Hauptgrund liegt darin, dass die traditionelle Pflicht der Sicherheitsgarantie beschränkte Schutzobjekte hat, und diese Schutzobjekte entweder vertraglich oder quasi-vertraglich mit dem Pflichtträger verbunden sind, während die Schutzobjekte im Internet unbeschränkt sind und häufig mit dem ISP gar keine Verbindung haben. ${ }^{614}$ Durch eine Gefährdungshaftung würden dem ISP zu viele Pflichten auferlegt. ${ }^{615}$

Ein anderer Grundsatz der Gefährdungshaftung könnte auf die Situation der Haftung des ISP angewendet werden, um das ebengenannte Gegenargument zurückweisen. Der Grundgedanke der Gefährdungshaftung liegt darin, wem die Vorteile gebühren, der soll auch die Nachteile tragen. ${ }^{616}$ Der ISP bietet der Öffentlichkeit eine Plattform, um ihre Meinungsfreiheit zu entfalten. Weil diese Dienstleistung für die Gesellschaft nützlich ist, ist das Verhalten des ISP gesellschaftlich erwünscht, auch wenn die Dienstleistung Rechtsverletzungen herbeibringen könnte. Hat der ISP von dieser gefährlichen Dienstleistung wirtschaftliches Interesse bekommen, muss er dann für die entstehende Rechtsverletzung Gefährdungshaftung tragen. In diesem Fall müssen der ISP und der Verletzte vorab kein rechtliches Verhältnis haben.

Das einzige Problem besteht nur bei der Art der Gefährdungshaftung. Gemäß $\$ 37$ Delikthaftungsgesetz soll der Pflichtträger, hier also der ISP, ergänzend haften. Das heißt, der Rechtsverletzte muss erst zu dem unmittelbaren Rechtsverletzer gehen, um Schadensersatz zu verlangen. Erst wenn der unmittelbare Rechtsverletzer nicht gefunden werden kann, oder wenn der Schadensersatz von dem unmittelbaren Rechtsverletzer nicht geleistet werden kann, haftet der Pflichtträger der Sicherheitsgarantie als zusätzlicher Schuldner für die Rechtsverletzung oder für den Rest des Schadensersatzes. Die ergänzende Haftung vom $\$ 37$ Delikthaftungsgesetz ist offensichtlich mit der gesamtschuldnerischer Haftung des ISP im $\$ 36$ Delikthaftungsgesetz nicht zu vereinbaren.

$\mathrm{Zu}$ überlegen ist es aber, ob eine ergänzende Haftung für den ISP doch vernünftiger als die gesamtschuldnerische Haftung ist, weil in diesem Fall der Rechtsverletzte zuerst zum unmittelbaren Rechtsverletzer gehen muss, bevor er

613 Vgl. Liu Wenjie, Peking University Law Journal 2012, No. 2, 395.

614 Mei Xiaying/Liu Ming, Science of Law 2013, No. 2, 82, 89.

615 Mei Xiaying/Liu Ming, Science of Law 2013, No. 2, 82, 89.

616 Zhang Wei, Law Science Magazine 2006, No. 3, 91, 92. 
den ISP in Anspruch nimmt. Diese Vorgehensweise ermöglicht es, die Rechtsverletzungen von der Quelle an zu vermindern.

\section{c) Die Auslegung der Kenntnisnahme i.S.v. \$ 36 Delikthaftungsgesetz}

Für die Beurteilung der Haftung des ISP ist der Zeitpunkt entscheidend, wann der ISP Kenntnis von der Rechtsverletzung erlangt. ${ }^{617} \$ 36$ Delikthaftungsgesetz hat nicht deutlich gesagt, ob das Wort Kenntnisnahme „positiv kennen“ oder auch „kennen sollen“ bzw. „fahrlässige Unkenntnis“ bedeutet. Darüber gab es in der Literatur intensive Diskussionen. ${ }^{618}$

Jetzt ist es aber in der Rechtsprechung und der Literatur allgemein anerkannt, dass Kenntnisnahme „positiv kennen“ und „kennen sollen“ bedeutet. ${ }^{619}$ Das heißt, die Pflicht des ISP im Sinne von $\$ 36$ Delikthaftungsgesetz entsteht, nicht nur wenn der ISP über die Rechtsverletzung deutlich weiß, sondern auch wenn er darüber wissen sollte.

Diese Meinung hat die Beurteilung der Kenntnisnahme des ISP über die Rechtsverletzung aber nicht großermaßen erleichtert, weil die Beurteilung des „kennen sollen“ immer noch eine schwierige Aufgabe ist. Inwiefern der ISP über die Rechtsverletzung wissen soll, ist mit seiner Sorgfaltspflicht eng verbunden. Diese Sorgfaltspflicht spiegelt die öffentlichen Politik bzw. die legislative Orientierung der Regierung an der Entwicklung des Internetdienstes wider. ${ }^{620}$ Bei der Auslegung soll ein Abwägungspunkt zwischen der gesunden Entwicklung der Internetindustrie und dem Schutz der Persönlichkeitsrechte gefunden werden. ${ }^{62}$ Mit diesem Hauptgedanken werden im Folgenden einige Kriterien für die Beurteilung der Sorgfaltspflicht des ISP über die Rechtsverletzung diskutiert.

\section{aa) Die Sorgfaltspflicht des ISP als eine normale rationale Person für alle Informationen}

ISP soll bei allen auf seiner Webseite erschienenen Informationen eine allgemeine Sorgfaltspflicht wie eine normale rationale Person haben. ${ }^{622}$ Für die Beurteilung

617 Mei Xiaying/Liu Ming, Science of Law 2013, No. 2, 82, 86.

618 Zhou Hua, Journal of China Three Gorges University (Humanities \& Social Sciences) 2012, No. 5, 87, 88.

619 Chen Jinchuan, Journal of Law Application 2012, No. 9, 25, 30.

620 Xue Hong, China Copyright 2011, No. 5, 36, 38.

621 Chen Jinchuan, Intellectual Property 2011, No. 2, 56, 58.

622 Chen Jinchuan, Intellectual Property 2011, No. 2, 56, 58; siehe auch Cheng Yan, Internet Law Review 2012, No. 1, 201, 208. 
des „wissen sollen“ wird „Red flag standard“ aus den USA von der Literatur vorgeschlagen, und von der chinesischen Literatur allgemein anerkannt. „Red flag standard" ist aus dem legislativen Bericht über DMCA von dem Senat und Repräsentantenhaus der Vereinigten Staaten heraufgekommen, um zu beurteilen, ob der ISP die Rechtsverletzung im Internet wissen soll oder nach Kenntnisnahme bezüglicher Sachverhalte die Rechtsverletzungen erkennen soll. ${ }^{623}$

Der Inhalt des „Red flag standard“ lautet: wenn eine Rechtsverletzung so offensichtlich ist, dass es wie eine grelle rote Fahne vor dem Gesicht des ISP flattert, damit jede Person, die unter gleichen Umstände Sorgfaltspflichten hat, die Rechtsverletzung einsehen kann. In diesem Fall soll der ISP rechtzeitig Maßnahmen vornehmen, um die Rechtsverletzung zu stoppen, auch wenn der Rechtsverletzte über die rechtsverletzende Tatsache den ISP nicht informiert. Sonst muss der ISP wegen Schuld für die Rechtsverletzung gesamtschuldnerisch haften. ${ }^{624}$

\section{bb) Spezielle Sorgfaltspflicht des ISP für die in besonderem Bereich der Webseite erscheinenden Informationen}

Auf einige Bereiche der Webseite soll der ISP besondere Aufmerksamkeit lenken. Dazu gehören die Bereiche, in denen die vom ISP aufgestellten Rankings oder von ihm empfohlenen Themen stehen. ${ }^{625}$ Aufgrund von $\$ 12$ Abs. 2 Nr. 1 „Regelung einiger Fragen über die Gesetzesanwendung auf die Beurteilung der Fälle über den zivilrechtlichen Rechtsstreit wegen Verletzung des informationellen Verbreitungsrechts im Internet" gehören zu den Bereichen sogar die ganze Homepage oder die Hauptseite, die offensichtlich vom ISP kontrolliert werden.

Die Informationen in diesen Bereichen haben meistens eine hohe Klickrate, hohe Bekanntheit, hohe Beliebtheit über das Thema und gewinnen hohe Aufmerksamkeit in der Gesellschaft. ${ }^{626}$ Sie verlangen deswegen mehr Sorgfaltspflichten von dem ISP. Über die in diesen Bereichen aufgetauchten Rechtsverletzungen soll der ISP normalerweise Kenntnis haben.

623 Shi Xueqing/Wang Yong, Intellectual Property 2009, No. 2, 23, 27.

624 Wang Qian, Studies in Law and Business 2008, No. 4, 42; Peng Xinghong, Journal of Hebei North University (Social Science Edition) 2013, No. 2, 76, 77; Li Yifei, Journal of Northwest University (Philosophy and Social Sciences Edition) 2010, No. 5, 67, 71; Wu Handong, China Legal Science 2011, No. 2, 38, 43; Chen Jinchuan, Journal of Law Application 2012, No. 9, 25, 30.

625 Wu Handong, China Legal Science 2011, No. 2, 38, 43.

626 Dai Yingjie, Journal of Zhejiang Institute of Media and Communications 2011, 37, 40. 


\section{cc) Die Qualifikation des ISP entscheidet über seine Kompetenz, Rechtsverletzungen zu entdecken.}

Für die Beurteilung der Sorgfaltspflicht des ISP soll seine Kompetenz zur Entdeckung der Rechtsverletzung berücksichtigt werden. Dazu gehört nicht nur die allgemeine Kompetenz einer normalen vernünftigen Person, sondern auch die fachliche Kompetenz als Dienstanbieter. ${ }^{627}$ Hier spielt dann die Größe und die Qualifikation eine Rolle. Der Provider, der eine große kommerzielle Webseite betreibt hat, bei der Verwaltung der Informationen normalerweise eine größere Kompetenz als der Provider, der eine kleine unbekannte unkommerzielle Webseite betreibt. ${ }^{628}$ Er hat deswegen normalerweise auch eine größere Kompetenz, um Rechtsverletzungen auf den Webseiten zu entdecken.

Ob ein ISP die Kompetenz hat, Rechtsverletzungen zu entdecken, muss er selbst beweisen. Aufgrund von $₫ 8$ Abs. 3 „Regelung einiger Fragen über die Gesetzesanwendung auf die Beurteilung der Fälle über den zivilrechtlichen Rechtsstreit wegen Verletzung des informationellen Verbreitungsrechts im Internet" sollen die Gerichte den ISP nicht als schuldig verurteilen, wenn er beweisen kann, dass es ihm nach der Benutzung von vernünftigen und praktischen technischen Maßnahmen immer noch schwierig ist, die von dem Internetnutzer vorgenommene Rechtsverletzung zu entdecken.

Anders formuliert soll der ISP, der die Kompetenz zur Entdeckung der Rechtsverletzung hat, aktiv passende Maßnahmen vornehmen, um die Rechtsverletzung zu entdecken. ${ }^{629}$ Sonst besteht die Gefahr, seine Sorgfaltspflicht zu verletzen.

\section{dd) Die ISP in China haben wegen der Verwaltungsregelungen größere Möglichkeiten, um Rechtsverletzungen zu entdecken}

In China ist es zwar allgemein anerkannt, dass die ISP keine allgemeine Überwachungspflicht auf alle Inhalte hat. Aber das Verwaltungsrecht verlangt, dass es auf der Internetseite z.B. keine reaktionären oder pornographischen Inhalte

627 Chen Jinchuan, Intellectual Property 2011, No. 2, 56, 58.

$628 \$ 9$ Abs. 1, Nr. 1 „Regelung einiger Fragen über die Gesetzanwendung auf die Beurteilung der Fälle über den zivilrechtlichen Rechtsstreit wegen Verletzung des informationellen Verbreitungsrechts im Internet" vom chinesischen Obersten Volksgerichtshof.

$629 \$ 9$ Abs. 1, Nr. 4 „Regelung einiger Fragen über die Gesetzanwendung auf die Beurteilung der Fälle über den zivilrechtlichen Rechtsstreit wegen Verletzung des informationellen Verbreitungsrechts im Internet" vom chinesischen Obersten Volksgerichtshof. 
geben darf. Dieses Verlangen ermöglicht bzw. zwingt den ISP, alle von dem Internetnutzer hochgeladenen Inhalte zu kontrollieren. ${ }^{630}$

„Die Methode zur Verwaltung der Informationsdienste im Internet" hat in $\$ 15$ geregelt, dass der ISP die beleidigenden, verleumderischen und anderen rechtsverletzenden Informationen nicht herstellen, kopieren, veröffentlichen und verbreiten darf. Gemäß $\$ 16$ dieser Methode soll der ISP die Verbreitung der im $\$ 15$ geregelt Inhalte sofort beenden, das Protokoll über diese Inhalte aufbewahren und an die zuständige Behörde berichten, wenn er diese Inhalte entdeckt. ${ }^{631}$ Das heißt, das chinesische Verwaltungsrecht impliziert, dass der ISP eine allgemeine Prüfungspflicht der Inhalte hat, die auf seiner Webseite erscheinen. Der ISP in China hat deswegen größere Möglichkeiten, die offensichtliche Rechtsverletzung im Internet zur Kenntnis zu nehmen. ${ }^{632}$

\section{ee) Die Größe der Sorgfaltspflicht des ISP kommt auch auf das dem Inhalt bezüglichen wirtschaftlichen Interesse an}

Gemäß $₫ 11$ Abs. 1 „Regelung einiger Fragen über die Gesetzesanwendung auf die Beurteilung der Fälle über den zivilrechtlichen Rechtsstreit wegen Verletzung des informationellen Verbreitungsrechts im Internet" soll das Gericht den ISP auf höhere Sorgfaltspflicht verurteilen, wenn er unmittelbares wirtschaftliches Interesse von dem rechtsverletzenden Werk bekommt, das von seinem Nutzer hochgeladen ist. Das unmittelbare wirtschaftliche Interesse besteht gemäß $\$ 11$ Abs. 2 dieser Regelung, wenn der ISP Profit von der Werbung bekommt, die spezifisch auf dem rechtsverletzenden Werk liegt; oder wenn der ISP anderes wirtschaftliches Interesse hat, das mit dem Werk spezifisch verbindlich ist. Die allgemeinen Werbung- oder Dienstgebühren, die wegen des Anbietens des Internetdienstes allgemein von dem ISP gesammelt werden, gehören nicht zu dem unmittelbaren wirtschaftlichen Interesse. ${ }^{633}$

Obwohl diese Vorschriften besondere Regelungen vom Urheberrecht sind, können sie meiner Meinung nach auch für den Fall zur Beurteilung der Sorgfaltspflicht des ISP bei dem Rechtsstreit über Persönlichkeitsrecht gelten, weil der Hintergedanke hauptsächlich darin liegt, „wem die Vorteile gebühren, der soll auch die Nachteile tragen“. Für den Fall der Persönlichkeitsrechtsverletzung,

630 Peking Erstes Mittleres Volksgericht, Urt. v. 28.10.2010 - (2010) yi zhong min chu zi di 3028 hao.

631 Wu Bo, S. 10.

632 Liang Zhiwen, Electronics Intellectual Property 2011, No. 9, 33, 36.

$633 \S 25$ „Richtlinie zur Behandlung des Rechtsstreits über Urheberrechtsverletzung im Internet (Probe-Version)“ vom Peking Oberen Volksgericht. 
Menschenfleischsuche beispielsweise, heißt es dann, wenn der ISP von der Menschenfleischsuche unmittelbares wirtschaftliches Interesse bekäme, hätte er eine höhere Sorgfaltspflicht, um die Inhalte der Menschenfleischsuche zu kontrollieren, um weiterhin die Rechtsverletzungen während der Suche zu vermeiden. ${ }^{634}$

\section{ff) Volle Sorgfaltspflicht des ISP wegen ICP ähnlichem Verhalten}

Gemäß $₫ 9$ Abs. 1 Nr. 3 „Regelung einiger Fragen über die Gesetzesanwendung auf die Beurteilung der Fälle über den zivilrechtlichen Rechtsstreit wegen Verletzung des informationellen Verbreitungsrechts im Internet" wird eine Kenntnisnahme des ISP über die Rechtverletzung vermutet, wenn er die rechtsverletzenden Inhalte aktiv ausgesucht, editiert, geändert oder empfohlen hat. ${ }^{635}$

Hier wird eine Änderung der rechtswidrigen Inhalte vom ISP als eine ausreichende Voraussetzung seiner Kenntnisnahme über die Rechtsverletzung behandelt. Für die gleichen Fälle würde der ISP in Deutschland wegen der Änderung der rechtswidrigen Inhalte ein ICP werden, weil er durch die Änderung die fremden Inhalte zu Eigen gemacht hat.

Die Haftung des ICP als ein ISP ist in China praktischer, weil es erstens im chinesischen Recht kein „Zueigenmachen“ gibt; es zweitens kaum Regelungen über die Haftung des ICP gibt, während die Haftung des ISP vom Delikthaftungsgesetz und die oben genannte Regelung deutlich geregelt ist. Übrigens führt es aus der Perspektive des Rechtsverletzten zum gleichen Ergebnis, egal ob der Provider als ICP oder ISP haftet, weil der ISP in diesem Fall nach der herrschenden Meinung als Mittäter gesamtschuldnerisch haftet. Das bedeutet, der Verletzte kann ebenso wie gegen den ICP direkt gegen den ISP vor Gericht klagen, und ggf. von ihm vollen Schadensersatz verlangen.

\section{gg) Höhere Sorgfaltspflicht des ISP für die von dem gleichen Internetnutzer begangene wiederholte Rechtsverletzung}

Für die wiederholte Rechtsverletzung von dem gleichen Internetnutzer wird von dem ISP mehr Sorgfaltspflicht erwartet. Bei einer wiederholten Rechtsverletzung wird häufig die Kenntnisnahme des ISP vermutet. ${ }^{636}$ Um eine Pflichtverletzung

634 Qian, Yuwen/Zhang, Jialin, Intellectual Property 2013, No. 2, 44, 46.

635 Vgl. Yang Lixin, Journal of National Prosecutors College 2010, No. 2, 3, 9.

$636 \S 9$ Abs. 1, Nr. 6 „Regelung einiger Fragen über die Gesetzanwendung auf die Beurteilung der Fälle über den zivilrechtlichen Rechtsstreit wegen Verletzung des informationellen Verbreitungsrechts im Internet" vom chinesischen Obersten Volksgerichtshof. 
zu vermeiden, muss er geeignet Maßnahme vornehmen, die wiederholte Rechtsverletzung von dem gleichen Internetnutzer zu vermeiden oder sie rechtzeitig zu entdecken.

\section{Unter bestimmten Umstände haftet der ISP als Gehilfe oder Anstifter}

Wenn der ISP den Internetnutzer bei rechtswidriger Handlung hilft oder anstiftet, soll er mit dem unmittelbaren Rechtsverletzer gesamtschuldnerisch haften. Gemäß $₫ 7$ „Regelung einiger Fragen über die Gesetzesanwendung auf die Beurteilung der Fälle über den zivilrechtlichen Rechtsstreit wegen Verletzung des informationellen Verbreitungsrechts im Internet" gehören zur Anstiftung die Verhalten des ISP, den Internetnutzer sprachlich, durch aktive Technikunterstützung, durch Belohnung usw. zu verleiten oder zu ermuntern, rechtswidrige Handlung zu unternehmen. Der ISP ist gemäß diesem Paragraph Gehilfe, wenn er weiß oder wissen soll, dass der Internetnutzer mit der Benutzung seines Dienstes rechtswidrige Handlung vornimmt, aber keine nötige Maßnahmen wie Löschen, Sperren, Verlinken-Abbrechen vorgenommen sondern Technikunterstützung gegeben hat.

Die Haftung des ISP als Gehilfe oder Anstifter macht nach chinesischem Recht kaum Sinn, weil der ISP in beide Fällen offensichtlich von Anfang an über die Rechtsverletzung weiß, und aus diesem Grund mit dem unmittelbaren Verletzer nach $₫ 36$ Abs. 3 Delikthaftungsgesetz gesamtschuldnerisch haften soll, was zu dem gleichen Ergebnis führt, wie wenn er als Gehilfe oder Anstifter haftet, während für die zwei Haftungen noch extra der bösgläubige Vorsatz bewiesen werden muss. Der Beweis von „kennen“ oder „kennen sollen“ ist offensichtlich einfacher als der vom Vorsatz zu helfen oder anzustiften. 


\section{Kapitel. Schutz des Rechtsverletzten}

Die Frage, wann eine Menschenfleischsuche rechtswidrig ist und wann die Teilnehmer, einschließlich des Informationsberichters, des Informationssammlers, des Veranlassers, des Kommentators, des ICP und des ISP, haften müssen, wurde bereits diskutiert. Jetzt wird die Frage, wie der Rechtsverletzte vor den Rechtsverletzungen geschützt werden kann, bzw. was für Ansprüche er gegen die Verletzer hat, behandelt. Darüber hinaus wird auch die Frage beantwortet, wie man rechtswidrige Menschenfleischsuche und die dadurch entstehenden Rechtsverletzungen von der Quelle an vermeiden kann und wie die Zielperson vorbeugend vor den Rechtsverletzungen geschützt werden kann. 



\section{$\$ 11$ Maßnahmen zum Schutz des Rechtsverletzten gegen den rechtsverletzenden Internetnutzer}

\section{Rechtsansprüche gegen den rechtsverletzenden Internetnutzer}

\section{Nach deutschem Recht}

a) Beseitigung- und Unterlassungsanspruch

Ist eine Rechtsverletzung des Persönlichkeitsrechts begründet, hat der Rechtsverletzte gemäß $₫ 1004$ Abs. 1 Satz 1 BGB den Anspruch auf Entfernung der rechtsverletzenden Inhalte und gemäß $₫ 1004$ Abs. 1 Satz 2 BGB den Anspruch auf Unterlassung einer Rechtsverletzung ähnlicher Art in der Zukunft.

\section{b) Schmerzensgeldanspruch}

Die Verletzung des Persönlichkeitsrechts kann auch durch eine Geldentschädigung wiedergutgemacht werden. Nach der Rechtsprechung des BGH handelt es sich bei einer Geldentschädigung aufgrund einer Verletzung des Persönlichkeitsrechts ,im eigentlichen Sinne nicht um ein Schmerzensgeld nach $₫ 847$ BGB a.F. (jetzt: $\$ 253$ Abs. 2 BGB n.F.)“, „sondern um einen Rechtsbehelf, der auf den Schutzauftrag aus Art. 1 und Art. 2 Abs. 1 GG zurückgeht“ “.637 Darunter wird vor allem die Genugtuung des Opfers berücksichtigt. ${ }^{638}$

Ferner liegt hier auch ein Präventionsgedanken vor. ${ }^{639}$ Das heißt, der Schmerzensgeldanspruch wird erst zugelassen, wenn die Gefahr besteht, dass ohne einen solchen Anspruch die Verletzungen der Persönlichkeit „ohne Sanktionen mit der Folge blieben, dass der Rechtschutz der Persönlichkeit verkümmern würde“. ${ }^{640}$

Deswegen wird ein Schmerzensgeldanspruch nicht in jedem Fall zugebilligt. ${ }^{641}$ Dazu müssen zwei Voraussetzungen erfüllt werden: zum einen muss es

637 BGH, NJW 2005, 58, 59.

638 BGH, NJW 2005, 58, 59; BGH, NJW 1996, 984; BVerfG, NJW 2000, 2187f.; siehe auch Schiemann in Staudinger, BGB $\$ 253$, Rn. 28ff. und 53ff.

639 BGH, NJW 2005, 58, 59.

640 BGH, NJW 2005, 58, 59; BGH, NJW 1996, 984.

641 Vgl. BGH, NJW 2005, 58, 59. 
sich um einen schwerwiegenden Eingriff des Persönlichkeitsrechts handeln; und zum anderen muss die Beeinträchtigung nicht in anderer Weise befriedigend ausgeglichen werden können. ${ }^{642}$

Um einen schwerwiegenden Eingriff zu beurteilen, müssen nach der Rechtsprechung des BVerfG einige Elemente berücksichtigt werden. Dazu zählen das Ausmaß der Verbreitung der verletzenden Aussagen, die Nachhaltigkeit der Fortdauer der Entschädigung, der Anlass und Beweggrund des Verletzers, sowie der Grad seines Verschuldens. ${ }^{643}$

\section{c) Schadensersatzanspruch}

Außer Schmerzensgeld kann der Verletzte gemäß $\$ 823$ Abs. 1 BGB Schadensersatz des wegen Persönlichkeitsrechtsverletzung entstehenden materiellen Schadens einschließlich Rechtsverfolgungskosten verlangen. ${ }^{644}$

\section{d) Antrag auf einstweilige Verfügung}

Jedoch sind Schadensersatz-, Schmerzensgeld-, Beseitigungs- und Unterlassungsansprüche nachträgliche Maßnahmen, die erst nach der Rechtsverletzung durch ein Gerichtsverfahren verwirklicht werden können. Wegen der Besonderheit der Persönlichkeitsrechtsverletzung im Internet ${ }^{645}$ ist ein vorbeugender Rechtsschutz effizienter, weil eine rechtzeitige Beendigung des laufenden Delikts während der Menschenfleischsuche die schnelle weitere Verbreitung der rechtsverletzenden Inhalte bzw. die Vertiefung der Rechtsverletzung vermeiden kann, während ein verletztes Persönlichkeitsrecht schlecht wiedergutgemacht werden kann. Beispielsweise kann eine offengelegte Intimsphäre nicht mehr wieder verdeckt werden.

$\$ 935$ ZPO hat durch die Regelung der einstweiligen Verfügung die Möglichkeit eines vorbeugenden Rechtsschutzes geschaffen. ${ }^{646}$ Nach dieser Vorschrift kann der Rechtsverletzte einen Antrag auf Erlass einer einstweiligen Verfügung stellen, ${ }^{647}$ um die Verletzung seines Persönlichkeitsrechts rechtzeitig zu verhindern. ${ }^{648} \mathrm{Zu}$ den erlaubten Verfügungsansprüchen gehören die Ansprüche auf Handlung und Unterlassung, die für die Rechtsverletzung im Fall der

642 BVerfG, NJW 2004, 591, 592.

643 BVerfG, NJW 2004, 591, 592.

644 Nink in Spindler/Schuster, BGB $\$ 823$, Rn. 48.

645 Siehe oben unter $\$ 2$ II.

646 Mayer in Vorwerk/Wolf, ZPO \$ 935, Rn. 8.

647 Kemper in Saenger, ZPO \$935, Rn. 11.

648 Kemper in Saenger, ZPO $\$ 935$, Rn. 11, 13; Mayer in Vorwerk/Wolf, ZPO \$ 935, Rn. 9. 
Menschenfleischsuche zum Schutz des Rechtsverletzens ausreichend sind ${ }^{649} \mathrm{Zu}$ dem zulässigen Verfügungsgrund gehört der Schutz des Persönlichkeitsrechts. ${ }^{650}$ Ein Antrag wäre begründet, wenn der Verletzte die Verfügungsansprüche und den Verfügungsgrund schlüssig behauptet und glaubhaft macht. ${ }^{651}$

\section{Nach chinesischem Recht}

\section{a) Allgemeine Regelungen über die Ansprüche}

Ist eine Rechtsverletzung des Persönlichkeitsrechts während Menschenfleischsuche nach chinesischem Recht begründet, hat der Rechtsverletzte gemäß $₫ 3$ i.V.m. $\$ 15$ Abs. 1 Delikthaftungsgesetz Ansprüche auf Entfernung der rechtsverletzenden Inhalte, Unterlassung weiterer Rechtsverletzung gleicher Art, Schadensersatz, Beseitigung der Wirkung, offizielle Entschuldigung und im Fall von Ehrverletzung auf Wiedergutmachung der Ehre. Diese Maßnahmen können gemäß $\$ 15$ Abs. 2 einzeln oder kombiniert verwendet werden.

\section{b) Schmerzensgeldanspruch}

Gemäß $₫ 22$ Delikthaftungsgesetz und $₫ 1$ Abs. 1 i.V.m. $₫ 8$ „Erklärung einiger Fragen über die Haftung für den immateriellen Schaden wegen zivilrechtlicher Rechtsverletzung" hat der Verletzte Anspruch auf Schmerzensgeld, wenn die Persönlichkeitsrechtsverletzung zu einem schwerwiegenden immateriellen Schaden führt. Die Beurteilung des schwerwiegenden immateriellen Schadens kann nur in jedem einzelnen Fall vom Gericht entschieden werden. ${ }^{62}$

Über die Höhe des Schmerzensgeldes müssen gemäß $\$ 10$ der oben genannten Erklärung die Schuld der Verletzer, die Konstellation des Vorgangs der Verletzung, das Ergebnis der Rechtsverletzung, das Profitieren des Verletzers durch

649 Mayer in Vorwerk/Wolf, ZPO \$ 935, Rn. 9.

650 Mayer in Vorwerk/Wolf, ZPO \$ 935, Rn. 9.

651 Kemper in Saenger, ZPO \$935, Rn. 10.

652 Vgl. Zhongshan Mitteleres Volksgericht, Urt. v. 25.11.2005 - (2005) zhong zhong fa min yi zhong zi di 1003 hao; Kaifeng Mitteleres Volksgericht, Urt. v. 19.12.2011 - (2011) bian min zhong zi di 1165 hao; Xi'an Mitteleres Volksgericht, Urt. v. 30.11.2012 - (2012) xi min er zhong zi di 02249 hao; Pukou Unteres Volksgericht, Urt. v. 12.12.2012 - (2012) pu min chu zi di 2125 hao; Shapingba Unteres Volksgericht, Urt. v. 17.9.2009 - (2009) sha fa min chu zi di 1800 hao; Nanjing Mittleres Volksgericht, Urt. v. 18.4.2013 - (2013) ning min zhong zi di 779 hao; Shanghai Zweite Mittleres Volksgericht, Urt. v. 5.5.2011 - (2010) hu er zhong min yi (min) zhong zi di 1593 hao. 
die Verletzung, die wirtschaftliche Fähigkeit des Verletzers zur Haftung und das Lebensniveau der Region des zuständigen Gerichts berücksichtigt werden.

\section{c) Antrag auf einstweilige Verfügung}

Einstweiliger Rechtsschutz ist in China ursprünglich zum Schutz des geistigen Eigentums entwickelt worden, ${ }^{653}$ und nach $\$ \$ 100 \mathrm{f}$. des neuen Zivilprozessgesetzes nur für vermögensrechtliche Ansprüche anwendbar. Jedoch hat ein unteres Volksgericht in der Provinz Jiangxi im März 2013 das erste Mal die einstweilige Verfügung offiziell auf den Fall über Persönlichkeitsrechtsverletzung analogisiert angewendet. ${ }^{65}$

Über die analoge Anwendung der einstweiligen Verfügung auf den Fall des Persönlichkeitsrechtsschutzes gibt es in der Literatur eine andere Meinung. Nach dieser Meinung ist eine Analogie im Persönlichkeitsrecht nicht möglich, weil die Rechtsverletzung des Vermögensrechts einfach bejaht oder verneint werden kann, während die Beurteilung der Rechtsverletzung des Persönlichkeitsrechts erst nach komplizierter Interessenabwägung möglich ist. ${ }^{655}$

Meiner Meinung nach sollte die analoge Anwendung möglich sein. Obwohl die Beurteilung der Persönlichkeitsrechtsverletzung in jedem einzelnen Fall durch Interessenabwägung erfolgt, ist sie nicht unbedingt schwieriger als die der Verletzung des Vermögensrechts. Dies ist besonders der Fall, wenn eine Verletzung des Persönlichkeitsrechts offensichtlich ist.

Die Bejahung der einstweiligen Verfügung ist möglich, wenn der Verletzte den Verfügungsgrund so glaubhaft begründet, dass eine Persönlichkeitsrechtsverletzung für jede Person offensichtlich ist. Für einen solchen Fall ist eine Interessenabwägung zwar notwendig, aber die Beurteilung ist nicht schwieriger als die von der Verletzung des Vermögensrechts.

Übrigens hat das untere Volksgericht in der Provinz Jiangxi den Prozess der einstweiligen Verfügung über das Vermögensrecht komplett übernommen. ${ }^{656}$

653 Zhu Wei, Legal Evening News, 10.05.2013, http://www.zxxk.com/article/245604. html (besucht am 04.04.2015).

654 Duchang Unteres Volksgericht, Beschl. v. 22.3.2013 - (2013) du wang chu zi di 2 hao; vgl. Guo Hongpeng/Huang Hui, Legal Daily, 25.03.2013, http://www.legaldaily.com. cn/index_article/content/2013-03/25/content_4308890.htm?node=5954 (besucht am 04.04.2015).

655 Zhu Wei, Legal Evening News, 10.05.2013, http://www.zxxk.com/article/245604. html (besucht am 04.04.2015).

656 Guo Hongpeng/Huang Hui, Legal Daily, 25.03.2013, http://www.legaldaily.com.cn/ index_article/content/2013-03/25/content_4308890.htm?node=5954 (besucht am 04.04.2015). 
Das heißt, der Antragsteller muss gemäß $\$ 101$ Zivilprozessgesetz eine Sicherheit leisten, um die Wahrheit seines Antrags zu garantieren. Dadurch wird das Recht des Äußernden ausreichend geschützt.

Der Verletzte muss die Rechtsverletzung des Persönlichkeitsrecht selbst beweisen und das Risiko tragen, wenn er den Antrag falsch gestellt hat. Eine einstweilige Verfügung für den Fall der Persönlichkeitsrechtsverletzung schadet keinem und ist zum Schutz des Verletzten vor Vertiefung der Rechtsverletzung erforderlich. Sie ist zu bejahen.

\section{Identifizierung des rechtsverletzenden Internetnutzers im Gerichtsprozess}

Um die oben genannten Ansprüche zu verwirklichen, muss der Verletzte Klage oder Antrag bei dem Gericht stellen. Dafür muss der Internetnutzer ersten einmal identifiziert werden.

\section{In Deutschland}

Gemäß $₫ 50$ ff. i.V.m. $\$ \$ 253$ Abs. 2 Nr. 1, 130 Nr. 1 ZPO muss die Bezeichnung des Beklagten oder des Antragsgegners - im Fall vom Antrag auf Erlass einer einstweiligen Verfügung - eindeutig sein ${ }^{657}$ Eine eindeutige Bezeichnung des Beklagten oder Antragsgegners soll mindestens den Klarnamen, Beruf und Anschrift beinhalten. ${ }^{658}$ Wie der Kläger oder Antragsteller diese Informationen hinter einem pseudonymen Internetnamen herausfinden kann, muss nach unterschiedlichen Fallgruppen diskutiert werden.

\section{a) Für den Fall der Verletzung des Rechts am eigenen Bild}

Der Rechtsinhaber des Bildes hat gemäß $₫ 101$ UrhG im Fall der offensichtlichen Rechtsverletzung einen Auskunftsanspruch gegen den Verletzer. Nach der herrschenden Meinung müssen die rechtsverletzenden Tätigkeiten nicht unbedingt im gewerblichen Ausmaß begangen worden sein. ${ }^{659}$ Nach der aktuellsten Rechtsprechung muss das Urheberrecht oder ein anderes nach dem

657 Hüßtege in Thomas/Putzo, $\$ 50$ Vorbem. Rn. 4; Lindacher, Münchener Kommentar zur ZPO, Vorbemerkung zu $\$ \$ 50$ ff., Rn. 12f.

658 Hüßtege in Thomas/Putzo, $\$ 50$ Vorbem. Rn. 4; Lindacher, Münchener Kommentar zur ZPO, Vorbemerkung zu $\$ \$ 50$ ff., Rn. 12f.

659 Spindler in Spindler/Schuster, UrhG $\$ 101$, Rn. 1; Czychowski in Fromm/Nordemann, UrhG $\$ 101$, Rn. 2, 11. 
Urheberrechtsgesetz geschütztes Recht auch nicht unbedingt in gewerblichem Ausmaß verletzt worden sein. ${ }^{60}$ Das heißt, ein Auskunftsanspruch gegen den Verletzer kann auch während der Menschenfleischsuche gegen den rechtsverletzenden Internetnutzer geltend gemacht werden.

Um einen rechtsverletzenden Internetnutzer im Fall von Verletzung des Rechts am eigenen Bild zu identifizieren, gibt es in der Praxis zwei Möglichkeiten.

\section{aa) Identifizierung durch Anmeldedaten}

Es ist oft der Fall, dass man sich mit Namen, Anschriften oder mindestens Pseudonym und Email-adresse anmelden muss, bevor man auf der Webseite Beiträge eintragen darf. In diesem Fall ist das Pseudonym häufig direkt mit den rechtsverletzenden Beiträgen verbunden. Würde es auf dieser Webseite pflichtig sein, sich mit realen Namen und realer Anschrift anzumelden, könnte der Verletzte direkt einen Anspruch auf die Auskunft der Anmeldedaten dem Provider verlangen. Die Pflicht der Auskunft bezieht sich nach der aktuellsten Entscheidung nicht nur auf Hostprovider, sondern auch auf Webseitenbetreiber. ${ }^{661}$

Geht es um Anmeldedaten muss $₫ 12$ Abs. 2 TMG angewendet werden. Aufgrund dieses Paragraphen ist der Hostprovider oder Webseitenbetreiber nicht zur Herausgabe der zur Bereitstellung des Telemediums erhobenen Anmeldedaten befugt, mit der Ausnahme, dass soweit eine Rechtsvorschrift dies erlaubt oder der Nutzer - was hier nicht in Rede steht - eingewilligt hat. ${ }^{662}$ Eine Ausnahme findet man jedoch in $\$ 14$ Abs. 2 TMG, aufgrund dessen der zuständigen Stellen auf Anordnung der Dienstanbieter im Einzelfall Auskunft über Bestandsdaten erteilen darf, soweit dies für Zwecke zur Durchsetzung der Rechte am geistigen Eigentum erforderlich ist. ${ }^{663}$ Die Anmeldedaten wie der Name und die Anschrift des Verletzers sind gemäß $\$ 14$ Abs. 1 TMG und $\$ 3$ Nr. 30 TKG zweifellos Bestandsdaten. ${ }^{64}$

Wegen der Verletzung des Rechts am eigenen Bild darf der Rechtinhaber Auskunft auf die Anmeldedaten des Verletzers vom Provider verlangen.

660 BGH, NJOZ 2013, 773.

661 OLG Dresden, ZUM-RD 2012, 536, 538.

662 BGH, K\&R 2014, 589, Rz. 9ff.

663 BGH, K\&R 2014, 589, Rz. 9ff.

664 BGH, NJOZ 2013, 773, 777. 


\section{bb) Identifizierung durch IP-Adresse}

Es ist auch oft der Fall, dass man ohne Anmeldung Beiträge eintragen kann. Noch häufiger ist es, dass man nicht pflichtig ist, mit realen Namen oder realer Anschrift anzumelden. In diesen Fällen ist es unmöglich, den Verletzer durch Anmeldedaten unmittelbar zu identifizieren. Aber eine Identifizierung des Verletzers unter Verwendung der dynamischen IP-Adresse, die er bei der Eintragung der rechtsverletzenden Beiträge vergibt hatte, ist immer noch möglich.

Für diesen Fall ist die oben genannte Gesetzanwendung aber fraglich, weil gemäß $\$ 14$ Abs. 2 TMG nur Bestandsdaten des Nutzers an einem Dritten ermittelt werden darf, aber es fraglich ist, ob dynamische IP-Adresse zur Bestandsdaten oder eher zur Verkehrsdaten im Sinne von $₫ 3$ Nr. 30 TKG gehört. Verkehrsdaten sind nach der Begriffsbestimmung des $₫ 3$ Nr. 30 TKG Daten, die bei der Erbringung eines Telekommunikationsdienstes erhoben, verarbeitet oder genutzt werden. Im Gegensatz handelt es sich bei Bestandsdaten nach der Legaldefinition des $\$ 3$ Nr. 3 TKG um Daten eines Teilnehmers, die für die Begründung, inhaltliche Ausgestaltung, Änderung oder Beendigung eines Vertragsverhältnisses über Telekommunikationsdienste erhoben werden.

Die Frage, ob dynamische IP-Adresse zur Bestandsdaten oder Verkehrsdaten gehört, ist schon immer umstritten und unklar gewesen. Die Aufsichtsbehörden für den Datenschutz vertrat eine vermittelnde Ansicht und sah in der IP-Adresse sowohl ein Bestands- als auch Verkehrsdatum. ${ }^{665}$ Der BGH hatte in der „Sommer unseres Lebens"-Entscheidung ${ }^{666}$ die Auffassung vertreten, IP-Adressen seien Bestandsdaten, ${ }^{667}$ weil sie zum einen nur Auskunft über den Namen des Anschlussinhabers geben würden, und sie zum anderen keine Angaben beinhalten, worüber und wie lange kommuniziert wurde. ${ }^{668} \mathrm{In}$ der Literatur herrscht die Meinung, dass sich dynamische IP-Adresse offensichtlich um personenbezogenes Verkehrsdatum handelt, denn sie Anknüpfungspunkt für die Darstellung ist, welche Informationen mittels eines Rechners bzw. über den Anschluss abgerufen wurden. ${ }^{669}$

Der Beschluss vom BGH am 19. 4. 2012 hat jedoch die Diskussion zu Ende gebracht, und die Schlussfolgerung getroffen, dass sich die IP-Adresse um

665 Karg, MMR-Aktuell 2011, 315811.

666 BGH, MMR 2010, 565.

667 Karg, MMR-Aktuell 2011, 315811.

668 BGH, MMR 2010, 565.

669 Karg, MMR-Aktuell 2011, 315811. 
Verkehrsdatum handelt. ${ }^{670}$ Eine dynamische IP-Adresse ist nach der Meinung des BGH keinem bestimmten Nutzer dauerhaft zugeordnet, sondern wird unterschiedlichen Nutzern jeweils nur für eine Sitzung (dynamisch) zugeteilt. ${ }^{671}$ Eine Verknüpfung der dynamischen IP-Adresse mit dem Nutzer, dem sie zu einem bestimmten Zeitpunkt zugewiesen war, ist daher nur unter Verwendung der jeweils hierzu gespeicherten Verkehrsdaten wie des Datums und der Uhrzeit der Verbindung möglich. ${ }^{672}$

Als Ergebnis des Falles, wenn der Verletzer nur durch Verwendung dynamischer IP-Adresse identifiziert werden kann, muss der Verletzte gemäß $\$ 101$ Abs. 9 S. 1 UrhG eine vorherige richterliche Anordnung über die Zulässigkeit der Verwendung der Verkehrsdaten beantragen.

\section{b) Für den Fall der Verletzung des Persönlichkeitsrechts allgemein}

Weiter wird diskutiert, ob die Situation der Verletzung des Rechts am eigenen Bild auch auf die Situation der Verletzung des allgemeinen Persönlichkeitsrechts angewendet wird.

Wie oben bereits erwähnt, dürfen für die Bereitstellung von Telemedien erhobene personenbezogene Daten für andere Zwecke nur verwendet werden, soweit eine Rechtsvorschrift dies erlaubt oder der Nutzer - was hier nicht in Rede steht eingewilligt hat ( $\$ 12$ Abs. 2 TMG). Die Ermächtigung besteht im Fall der Verletzung des Rechts am eigenen Bild im Rahmen der Durchsetzung der Rechte am geistigen Eigentum in $\$ 14$ Abs. 2 TMG. Eine gleiche Regelung zum Schutz der allgemeinen Persönlichkeitsrechte findet man innerhalb TMG jedoch nicht. ${ }^{673}$

Ein Auskunftsanspruch könnte aber aus Treu und Glauben im Sinne von $₫ 242$ BGB ausgeleitet werden. Liegt auf der Webseite eine Persönlichkeitsrechtsverletzung vor, muss der Webseitenbetreiber unabhängig von seiner Schuld als Störer haften. ${ }^{674}$ Daraus entstehen die Ansprüche der Verletzten auf Unterlassung und Löschung persönlichkeitsverletzender Einträge. Wegen dieser Ansprüche entsteht zwischen dem Verletzten und dem Betreiber oder dem Provider ein gesetzliches Schuldverhältnis. ${ }^{675}$ Nach der ständigen Meinung des BGH besteht ein Auskunftsanspruch

670 BGH, NJOZ 2013, 773, 777.

671 BGH, NJOZ 2013, 773, 777.

672 BGH, NJOZ 2013, 773, 777; vgl. OLG Hamburg, NJOZ 2010, 1222 = ZUM 2010, 893, 898.

673 BGH, K\&R 2014, 589.

674 OLG Dresden, ZUM-RD 2012, 536, 538.

675 OLG Dresden, ZUM-RD 2012, 536, 538. 
des Berechtigten gegen den Verpflichteten in jedem Rechtsverhältnis, „,in dem der Berechtigte in entschuldbarer Weise über Bestehen und Umfang seines Rechtes im Ungewissen und der Verpflichtete unschwer zur Auskunftserteilung in der Lage

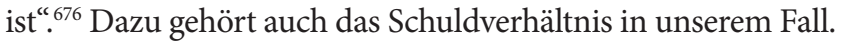

Diese Meinung wurde vom BGH in seiner aktuellsten Entscheidung am 1. 7. 2014 bejaht. ${ }^{677}$ Nach seiner Meinung hat der Provider im Fall der Persönlichkeitsrechtsverletzung eine "Auskunftspflicht“ ${ }^{678}$ Jedoch hat er die Anspruchsmöglichkeit des Verletzten abgelehnt, weil der Provider doch „nicht zur Herausgabe der zur Bereitstellung des Telemediums erhobenen Anmeldedaten befugt ist" ${ }^{679}$

Der BGH geht davon aus, dass eine Erlaubnis zur Auskunft der Nutzerdaten durch Rechtsvorschrift außerhalb des Telemediengesetzes lediglich dann in Betracht kommt, wenn sich eine solche Vorschrift ausdrücklich auf Telemedien bezieht. ${ }^{680}$ Dies scheidet dann den aus Treu und Glauben ( $\$ 242$ BGB) hergeleiteten allgemeinen Auskunftsanspruch aus, weil sich $\$ 242$ BGB nicht ausdrücklich auf Telemedien bezieht.

Der BGH hat weiterhin die Möglichkeit eine analoge Anwendung von $\$ 14$ Abs. 2 TMG, $\$ 15$ Abs. 5 Satz 4 TMG zur Durchsetzung der Rechte am geistigen Eigentum ebenfalls ausgeschieden, da es seiner Meinung nach an einer planwidrigen Regelungslücke fehlt. ${ }^{681}$ Die Vorschriften dienen ausschließlich dem Schutz des geistigen Eigentums, um Innovation und kreatives Schaffen zu fördern, den Arbeitsmarkt zu entwickeln und die Wettbewerbsfähigkeit zu verbessern; Persönlichkeitsrechte werden hier nicht bezogen. ${ }^{62}$

Die Absage der analogen Anwendung wurde in der Literatur kritisiert. ${ }^{633}$ Der BGH geht selbst auch davon aus, dass seine Meinung wenig überzeugend ist. ${ }^{684}$

Meiner Meinung nach soll eine analoge Anwendung von $\$ 14$ Abs. 2 TMG, $\$ 15$ Abs. 5 Satz 4 TMG zur Durchsetzung der Rechte am geistigen Eigentum zulässig sein. Wie oben schon diskutiert, hat der BGH einen Auskunftsanspruch wegen Verletzung des Rechts am eigenen Bild zugesagt. Bei der Begründung

676 BGH, BGHZ 10, 385; BGHZ 126, 109, 113; BGHZ 149, 165, 175; OLG Dresden, ZUM-RD 2012, 536, 538.

677 BGH, K\&R 2014, 589.

678 BGH, K\&R 2014, 589, Rn. 6.

679 BGH, K\&R 2014, 589, Rn. 9.

680 Nach dem Gesetzeswortlaut von $₫ 12$ Abs. 2 TMG.

681 BGH, K\&R 2014, 589, Rn. 13ff.

682 BGH, K\&R 2014, 589, Rn. 15.

683 Klein, GRUR-Prax 2014, 539.

684 BGH, K\&R 2014, 589, Rn. 17. 
wurde allgemein anerkannt, dass die rechtsverletzenden Tätigkeiten nicht unbedingt im gewerblichen Ausmaß begangen worden sein, ${ }^{685}$ und das geschütztes Recht auch nicht unbedingt in gewerblichem Ausmaß verletzt worden sein müssen. ${ }^{686}$ Das bedeutet, dass der Schutzgegenstand nicht nur der wirtschaftliche Teil des Rechts am eigenen Bild als Urheberrecht ist, sondern auch lediglich der persönlichkeitsrechtliche Teil des Rechts am eigenen Bild als Persönlichkeitsrecht sein kann. Dies hat offensichtlich die Tür zur analogen Anwendung auf den Fall der allgemeinen Persönlichkeitsrechtsverletzung geöffnet.

Der BGH hat am Ende seiner Entscheidung am 1. 7. 2014 zugegeben, dass die Beschränkung der Ermächtigung zur Auskunftserteilung auf Inhaber von Rechten am geistigen Eigentum wenig nachvollziehbar und eine Ausweitung auf Persönlichkeitsrechtsverletzungen wünschenswert sein mag. ${ }^{677}$ Aber eine solche Regelung müsste seiner Meinung nach der Gesetzgeber treffen. ${ }^{68}$

Durch die Entscheidung des BGH wird ein allgemeiner Auskunftsanspruch gegen den Provider wegen Persönlichkeitsrechtsverletzung in Deutschland abgesagt. Außer dem Fall der Verletzung des Rechts am eigenen Bild kann ein Auskunftsanspruch nur noch verlangt werden, wenn sich das Persönlichkeitsrechtsverletzen, zum Beispiel in Form von Beleidigung oder Verleumdung, zu Straftat wandelt, und der Auskunftsanspruch dann zum Zweck der Strafverfolgung dient. ${ }^{689}$

\section{c) Zwischenergebnis}

In Deutschland hat der Verletzte Auskunftsanspruch gegen den ISP auf die Nutzerdaten des unmittelbaren Verletzers im Fall von Verletzung des Rechts am eigenen Bild oder in den Fällen von schwerwiegenden Persönlichkeitseingriffen, Beleidigungen oder Verleumdungen wie zum Beispiel, zu Zwecken der Strafverfolgung. ${ }^{690}$ Ein allgemeiner Auskunftsanspruch wegen Verletzung allgemeiner Persönlichkeitsrechte wird vom BGH ausdrücklich abgelehnt.

Jedoch hat der BGH durch seine Entscheidung die Diskussion über den allgemeinen Auskunftsanspruch nicht beendet. Auf einer Seite sind die Begründungen

685 Spindler in Spindler/Schuster, UrhG \$ 101, Rn. 1; Czychowski in Fromm/Nordemann, UrhG $\$ 101$, Rn. 2, 11.

686 BGH, NJOZ 2013, 773.

687 BGH, K\&R 2014, 589, Rn. 17.

688 BGH, K\&R 2014, 589, Rn. 17.

$689 \S 14$ Abs. 2 TMG.

690 Klein, GRUR-Prax 2014, 539, 541. 
zur Ablehnung des Auskunftsanspruch nicht überzeugend genug. In der Literatur gibt es schon kräftige Gegenmeinungen. Auf der anderen Seite hat der BGH in der Entscheidung auch selbst hingewiesen, dass eine Anerkennung des Auskunftsanspruchs nachvollziehbar und wünschenswert ist, nur nicht er sondern der Gesetzgeber dafür zuständig ist.

Es ist verständlich, dass sich der BGH nicht traut, einen allgemeinen Auskunftsanspruch anzuerkennen, weil dahinten doch die Sorge auf Beschränkung der Meinungsäußerungsfreiheit im Sinne von anonymen Äußerungen steht. ${ }^{691}$ Die weitere Entwicklung über dieses Thema ist in Deutschland zu erwarten.

Zurzeit ist es in Deutschland für den Verletzten ggf. einfacher und praktischer, direkt auf den ISP einzuwirken, ein Beseitigungs- und Unterlassungsanspruch im Rahme der Störerhaftung geltend zu machen ${ }^{692}$ Dies hilft jedoch nicht, die Verletzungen der Persönlichkeitsrechte von der Quelle an zu vermeiden.

\section{In China}

Um einen Zivilprozess in China durchzuführen, muss der Kläger gemäß $\$ 119$ Abs. 1 Nr. 2 Zivilprozessgesetz einen klaren Beklagten haben, gegen den er klagt. Ein klarer Beklagter bedeutet gemäß $\$ 121$ Abs. 1 Nr. 2 Zivilprozessgesetz, dass der Name, das Geschlecht, die Anschrift und der Beruf des Beklagten vorhanden sein müssen. Dies gilt gemäß $\$ 100 \mathrm{f}$. Zivilprozessgesetz auch für den Prozess auf Erlass einer einstweiligen Verfügung.

Bei dem Fall der Persönlichkeitsrechtsverletzung während der Menschenfleischsuche ist es am häufigsten, dass die wahren persönlichen Daten des Verletzers unbekannt sind. Nach chinesischem Recht besteht zwischen dem Verletzten und dem Provider entweder eine vertragliche oder eine gesetzliche Verbindung, aus der der Verletzte einen Auskunftsanspruch auf die persönlichen Daten des Internetnutzers verlangen kann. Um einen rechtsverletzenden Internetnutzer zu identifizieren muss der Verletzte vor dem Gericht klagen, weil nur das Gericht von Amts wegen die Identifizierung des Beklagten erwirken kann. Dies schafft eine Zwickmühle für den Verletzten.

Um dieses Problem zu lösen, haben die Gerichte in der Provinz Jiangxi eine neue Methode geschaffen. Gemäß $\$ 9$ „Leitlinie einiger Fragen über die Gesetzesanwendung für die Beurteilung der Fälle über Rechtsverletzung im Internet von dem Oberen Volksgericht in der Provinz Jiangxi (Probe-Version)" kann die IP-Adresse oder das Pseudonym des Beklagten vorläufig verwendet werden, um ein Vorverfahren

691 LG München, BeckRS 2013, 12855, in Anlehnung an BGH MMR 2009, 608, 612.

692 Uffeln/Günther, http://www.kanzlei-uffeln.de/ku/ (besucht am 04.04.2015), S. 6. 
vor dem offiziellen Gerichtsprozess einzuleiten. Während dieses Vorverfahrens kann der Kläger gemäß $₫ 64$ Abs. 2 Zivilprozessgesetzes beim Gericht beantragen, von Amts wegen die Registrierungsinformation oder IP-Adresse des Beklagten von dem ISP zu fordern. Gleichzeitig kann der Kläger bei der Behörde für die Sicherheitskontrolle im Internet beantragen, den Beklagten durch die IP-Adresse oder Registrierungsinformation zu identifizieren. Erst nachdem der Beklagte als eine bestimmte Person festgestellt wurde, beginnt der offizielle Gerichtsprozess. Sonst wird die Klage abgewiesen. Diese Methode ist zwar noch nicht vom höheren Gericht offiziell anerkannt, aber ihr begegnet jedoch auch keine Gegenmeinung.

\section{Ein kurzer Vergleich zwischen Deutschland und China}

Bei der Identifizierung des Verletzers haben Deutschland und China zwei unterschiedliche Wege genommen.

Um eine Klage oder eine einstweilige Verfügung gegen den Internetnutzer durchzuführen, muss sich der Verletzte in Deutschland erst an den ISP wenden, um gegen ihn einen Auskunftsanspruch zu verlangen. Hier soll der ISP nach seinen Kenntnissen entscheiden, ob eine offensichtliche Persönlichkeitsrechtsverletzung vorliegt. Wenn der ISP eine andere Meinung als der Verletzte hat, und deswegen eine Auskunftserteilung verneint, muss der Verletzte erst gegen den ISP klagen, um seinen Auskunftsanspruch zu verwirklichen, bevor er endlich eine Klage gegen den unmittelbaren Verletzer erheben kann.

Dieses Verfahren ist für den Verletzten viel unpraktischer als direkt einen Beseitigungs- oder Unterlassungsanspruch gegen den ISP zu stellen. Übrigens muss der ISP ein hohes Risiko für seine Entscheidung tragen, ob eine offensichtliche Persönlichkeitsrechtsverletzung vorliegt. Wird eine Verletzung von ihm irrtümlich bejaht, und die Kundendaten dem „Verletzten“ mitgeteilt, muss er wegen Pflichtverletzung i.S.v. $\$ 13$ Abs. 6 TMG haften. Verneint er eine offensichtliche Persönlichkeitsrechtsverletzung muss er an einem Gerichtverfahren auf Auskunftsanspruch gegen ihn teilnehmen. Durch dieses Verfahren werden die hohen Belastungen dem ISP gegeben. Der unmittelbare Verletzer, der die Verletzung verursacht, ist dagegen vom Verletzten schlecht erreichbar. Dem Verfahren dient es nicht, die Verletzungen von der Quelle an zu vermeiden.

Durch das Vorverfahren, in dem der unmittelbare Verletzer von Amts wegen ermittelt wird, ist in China eine Klage direkt gegen den unbekannten rechtsverletzenden Internetnutzer möglich. Der ISP ist dadurch von der Beurteilung der Persönlichkeitsrechtsverletzung befreit, und braucht sich nicht um die falsche Erteilung der Kundendaten sorgen, weil die Erteilung immer 
auf Verlangen des Gerichts durchgeführt wird. Das Gericht ist in diesem Fall der einzige, der die Persönlichkeitsrechtsverletzung beurteilt und der wegen seiner Fachkenntnisse die Richtigkeit der Beurteilung garantieren kann. Das Verfahren in China bzw. in der Provinz Jiangxi ermöglicht dem Verletzten einen besseren Weg, um den Rechtsanspruch direkt gegen den unmittelbaren Verletzer zu verlangen.

\section{Real-Name-System}

Real-Name-System bedeutet, dass die Internetnutzer mit ihren realen Namen und ID-Nummer oder Passnummer registriert sein müssen, bevor sie Beiträge im Internet veröffentlichen. Die Wahrheit der beim Registrieren eingegebenen Information wird durch Zusammenarbeit zwischen dem ISP und der Behörde der öffentlichen Sicherheit überprüft. Bei der Meinungsäußerung auf der Webseite darf der Internetnutzer ein Pseudonym benutzen. Aber nach Real-NameSystem steht hinter jeden pseudonymen Namen immer eine richtige Person, die für ihr online Verhalten verantwortlich sein soll.

Durch Real-Name-System wird das Problem der unbekannten Verletzer im Internet gelöst. Der Verletzte kann gegen den Beklagten direkt mit dem pseudonymen Namen vor Gericht klagen, weil die richtige Person hinter dem pseudonymen Namen durch Real-Name-System sofort herausgefunden werden kann.

Jedoch besteht für Real-Name-System auch die Gefahr, dass die Internetnutzer aus Furcht vor Repressalien oder sonstigen negativen Auswirkungen ihre Meinungen nicht mehr äußern, was zur Selbstzensur führt.

Real-Name-System ist ein heißes Thema in China. Deswegen wird die folgende Diskussion über Real-Name-System mit China anfangen.

\section{Real-Name-System in China}

\section{a) Die Entwicklung des Real-Name-System in China}

Am 21. Nov. 2001 hat das chinesische Bildungsministerium die „Regelung zur Verwaltung des Diensts des elektronischen schwarzen Brettes (BBS-Service) des Hochschulnetzwerkes“ erlassen. Aufgrund $\$ 9$ dieser Regelung sollen Hochschulen beim Anbieten eines elektronischen schwarzen Brettes das Real-Name-System benutzen. Das ist die früheste Regelung über Real-Name-System in China.

Im Jahr 2002 hat ein Journalistischer Professor Xiguang Li eine Rede gehalten, dass niemand in China anonym im Internet Einträge veröffentlichen soll. Diese Rede hat intensive Diskussionen über das Real-Name-System herbeigeführt. 
Viele Regelungen über Real-Name-System wurden damals entworfen und sind in Kraft getreten. ${ }^{693}$

Im Jahr 2004 begann die Webseite „Chinavalue.net“, Real-Name-System auf der Webseite durchzusetzen. Das war die erste kommerzielle Webseite in China, die das Real-Name-System verwendet hat.

Im selben Jahr hatte das Bildungsministerium den „Vorschlag über die Verstärkung der Verwaltung des internen Hochschulnetzwerkes“ erlassen, um das Real-Name-System im internen Hochschulnetzwerk einen Schritt weiter einzusetzen. Die Meinungsäußerung im internen Hochschulnetzwerk stand unter strenger Untersuchung und starken Beschränkungen. ${ }^{694}$

Am 1. 5. 2009 hatte das ständige Komitee des Volkskongresses der Stadt Hangzhou die "Administrative Verordnung zum Schutz der Informationssicherheit im Internet von der Stadt Hangzhou" erlassen. Gemäß dieser Verordnung mussten sich die Internetnutzer bei Meinungsäußerungen im Internet mit gültigen ID-Nummern registrieren. Das war die erste offizielle regionale Vorschrift in China, die das Real-Name-System festgelegt hatte. Aber weil die Verordnung nach der Erlassung zu stark kritisiert wurde, wurde sie nie wirklich durchgesetzt. ${ }^{695}$

Die am 16.12.2011 erlassene „Regelungen zur Verwaltung der Entwicklung des Mikrobloggings" hat auch verlangt, dass sich die Nutzer von Mikroblogging mit realen Namen registrieren sollen.

Am 26.3.2013 hat das General Büro des Staatsrats in der „Mittelung über die Verteilung der Aufgaben, um den Plan für institutionelle Reform und Transformation der Funktion des Staatsrates durchzusetzen" festgestellt, dass es die Aufgabe der Regierung im Jahr 2014 ist, das Real-Name-Registrieren-System zu verbreiten und durchzusetzen. Diese Aufgabe wird von dem Ministerium für Industrie und Informationstechnologie, National Büro für Internetinformation, Ministerium für öffentliche Sicherheit aufgenommen, und muss bis Ende Juni 2014 erledigt werden. ${ }^{696}$

Bis zu dieser Mitteilung wird die Durchsetzung des Real-Name-System in China fast festgestellt. Jedoch ist es immer notwendig, die Verhältnismäßigkeit des Real-Name-System zu überprüfen.

693 Lu Wei, Lanzhou Academic Journal 2012, No. 9, 161.

694 Wikipedia, http://en.wikipedia.org/wiki/SMTH_BBS (besucht am 04.04.2015).

695 http://it.people.com.cn/GB/42891/42894/11777005.html (besucht am 04.04.2015).

696 http://www.gov.cn/zwgk/2013-03/28/content_2364821.htm_(besucht am 04.04.2015). 


\section{b) Die Verhältnismäßigkeit des Real-Name-System}

\section{aa) Der legitime Zweck des Real-Name-System}

Außer dem oben genannten Zweck, dass der unmittelbare Verletzer durch RealName-System leichter identifiziert werden kann, und der Verletzte dadurch besser geschützt werden kann, lag der ursprüngliche Zweck des Real-Name-System auch oder mehr in der Kontrolle der Internetgewalt. Dazu gehören die im Internet umfangreich verbreiteten Handlungen wie Internet-Mobbing, Beschmutzung, Betrug, Verbrechen und anderes rechtswidriges Verhalten. ${ }^{697}$

Dieses Chaos kann nicht durch Selbstregulierung der Internetnutzer gelöst werden. Das Einsetzen der öffentlichen Macht ist nötig, um die Internetwelt wieder in Ordnung zu bringen. ${ }^{698} \mathrm{Zu}$ diesen Zwecken ist Real-Name-System als die passende Maßnahme ausgewählt worden, weil sich die Internetnutzer durch die Abschreckungsfunktion des Real-Name-System im Internet vernünftiger und vorsichtiger verhalten würden.

Das Real-Name-System dient zum legitimen Zweck.

\section{bb) Die Geeignetheit des Real-Name-System}

Hier wird diskutiert, ob das Real-Name-System geeignet ist, das Chaos im Internet wieder in Ordnung zu bringen.

Die Förderung wird hauptsächlich durch die Abschreckungsfunktion des Real-Name-System verwirklicht. Nicht zu negieren ist es, dass die Anonymität und Pseudonymität im Internet die Internetnutzer ermutigen, sich so zu verhalten wie sie es sich im realen Leben nicht trauen würden. Durch RealName-System wird jeder Internetnutzer mit seinem Verhalten im Internet eng verbunden. Besonders bei der Ausübung rechtswidrigen Verhaltens zwingt das Real-Name-System die Internetnutzer zu überlegen, dass sie wegen des rechtswidrigen Verhaltens genau wie im normalen Leben bestraft würden. Diese Abschreckungsfunktion würde schon eine Verminderung der rechtswidrigen Handlungen im Internet fördern.

Das Real-Name-System könnte von einem anderen Aspekt auch dem legitimen Zweck dienen, Klagen gegen den unmittelbaren rechtsverletzenden Internetnutzer zu erleichtern. Der Verletzer würde leichter identifiziert, was zur Folge hätte, dass der Verletzer für seine rechtswidrige Handlung im Internet haften müsste. Dies hilft auch die Rechtsverletzung von der Quelle an zu vermindern.

697 Yang Fuzhong, Studies in Law and Business 2012, No. 5, 32.

698 Gao Wenmiao, Lanzhou Academic Journal 2012, No. 3, 167, 168. 
Jedoch ist es fraglich, ob die Abschreckungsfunktion wirklich funktioniert. Das Real-Name-System braucht technische Unterstützung. Ein Programm muss entwickelt werden, um das Real-Name-System zu verwirklichen. Ein Programm kann leider niemals so perfekt bzw. lückenlos sein, damit es von keinem eingehackt werden kann. Andererseits würden die meisten Internetnutzer es bevorzugen, anonym zu bleiben. Darum würde es großen Bedarf geben, das Real-Name-System zu umgehen. Für diesen Bedarf würde es auch genug Hacker geben, ein Programm für die Umgehung des Real-Name-System zu programmieren.

Übrigens würde ein verständiger Internetnutzer, der schon weiß, dass sein Verhalten rechtswidrig sein würde, auf keinen Fall dieses Verhalten unter seinem eigenen realen Namen begehen. ${ }^{699}$ Ein Umweg würde von ihm gefunden werden. Im Gegenteil wäre es der normale Internetnutzer, der Abschreckung für seine sehr wahrscheinlich rechtsmäße Meinungsäußerung bekommt.

Das Real-Name-System würde seine erwartete Funktion nicht erreichen. ${ }^{700}$

\section{cc) Die Erforderlichkeit des Real-Name-System}

Hier soll diskutiert werden, ob ein milderer Weg zur Verfügung steht, Internetgewalt zu kontrollieren.

Die Abschreckungsfunktion des Real-Name-System funktioniert eigentlich dadurch, dass die Internetnutzer Angst haben sollen, leicht identifiziert zu werden, wenn sie rechtswidrige Handlungen begehen. Aber ohne Real-NameSystem ist es schon möglich, durch pseudonymen Name und IP-Adresse den Verletzer zu identifizieren. ${ }^{701}$ Das ist auch die üblichste Methode weltweit, um Verletzer oder Verbrecher im Internet zu identifizieren.

Der Unterschied zwischen IP-Identifizierung und Real-Name-SystemIdentifizierung ist nur, dass Real-Name-System-Identifizierung schneller sein könnte. Jedoch ist es möglich, durch andere Wege den Prozess zur Identifizierung des Verletzers zu beschleunigen. Die Gerichte in der Provinz Jiangxi haben durch ihre Praxis die Möglichkeiten erwiesen. ${ }^{702}$

Übrigens kann der Beklagte im offline Leben auch nicht immer sofort identifiziert werden. Es ist unmöglich, dass jeder Verletzte jeden Verletzer kennt. Eine

699 Xu Zhenzeng, Hebei Law Science 2012, No. 9, 166, 170.

700 Vgl. Yang Fuzhong, Studies in Law and Business 2012, No. 5, 32, 37-38.

701 Xu Zhenzeng, Hebei Law Science 2012, No. 9, 166, 171; Han, Ning, Legal Science Monthly 2012, No. 4, 3, 8 .

702 Siehe oben unter $\$ 11$ II 2. 
Reihe von Untersuchungen ist im offline Leben auch nötig, um den beklagten Verletzer zu identifizieren. Darum wäre es eine Überforderung für die Internetwelt, den Verletzer sofort finden zu müssen.

Außerdem steht schon z.B. das Notice-and-Take-Down-Verfahren zur Verfügung, damit der Verletzte eine Rechtsverletzung rechtzeitig abbrechen kann.

Um die Nicht-Erforderlichkeit des Real-Name-System zu erklären, hat ein Jurist ein Metapher erfunden: weil es Internetgewalt gäbe, müsse Real-NameSystem durchgeführt werden; nach dieser Theorie, müsse jeder schon seinen Namen, ID-Nummer auf die Stirn schreiben, weil es im offline Leben auch Gewalt geben könnte. ${ }^{703}$

Nach alledem ist ein Real-Name-System nicht erforderlich.

\section{dd) Die Angemessenheit des Real-Name-System}

Hier wird geprüft, ob die Nachteile des Real-Name-Systems völlig außer Verhältnis gegenüber den Vorteilen stehen.

\section{(1) Bewertung über die Abschreckungsfunktion des}

Real-Name-Systems

Das Real-Name-System könnte im Internet eine Situation schaffen, in der die Meinungsäußerung viel stärker als im offline Leben beschränkt wird, weil alle im Internet eingetragenen Inhalte gespeichert und zur Abrufung bereitgestellt werden. Wenn das Real-Name-System durchgeführt würde, könnte jedes Wort, das ein Internetnutzer im Internet eingetragen hat, unter seinem Namen aufgenommen werden und in der Zunft als Beweis gegen ihn verwendet werden. Im offline Leben wird auf keinen Fall jedes Gespräch aufgenommen.

Es ist vorstellbar, dass der Internetnutzer bei der Meinungsäußerung sehr vorsichtig sein wird und viel überlegen muss, um einen Fehler zu vermeiden, der in der Zukunft zu einer Haftung für ihn führen könnte. Die Abschreckungsfunktion erstreckt sich nicht nur auf die Äußerung der rechtswidrigen Inhalte, sondern auch auf die der rechtmäßigen Inhalte.

Besonders zu erwähnen ist es, dass die Abschreckungsfunktion des RealName-Systems auch dazu führen könnte, dass die Denunziation der Korruptionen durch das Internet nicht mehr oder seltener funktioniert, was in der letzter Zeit eine wichtige bzw. hauptsächliche Methode zur Ausübung des Aufsichtsrechts chinesischer Bürger über die Beamten ist. Das Aufsichtsrecht von den Bürgern über die Beamten ist gemäß $₫ 41$ chinesisches Verfassungsgesetz

703 Zhou Yongkun, Jinan Journal (Philosophy and Social Sciences) 2013, No. 2, 1, 5. 
ein Grundrecht der chinesischen Bürger. Die Daten zeigen, dass 80\% der Korruption in China unmittelbar durch anonyme Denunziation der Bürger aufgedeckt wurden.$^{704}$ Es herrscht die Meinung von einem Beamtem aus der Höchsten Staatsanwaltschaft, dass tatsächlich $100 \%$ der Korruption in China durch unmittelbare und mittelbare anonyme Denunziation der Bürger aufgedeckt sind. ${ }^{705}$ In der letzten Zeit ist die Häufigkeit der anonymen Online-Denunziation um das 8 fache gestiegen. ${ }^{706}$

Wenn das Real-Name-System durchgesetzt werden würde, würden anonyme Denunziationen schlecht möglich sein, weil der Denunziant Angst haben würde, dass er nach der Denunziation von demjenigen Beamten gerächt wird, bevor er aufgrund der Korruption seine Macht verliert. Das wäre offensichtlich eine Beschränkung des Aufsichtsrechts des Volkes. ${ }^{707}$

\section{(2) Real-Name-System erhöht das Risiko vom Durchsickern der persönlichen Informationen}

Die Hauptgefahr vom „Leak“ der Informationen kommt nicht von normalen Internetnutzern sondern aus den Hackerkreisen. Am Ende des Jahres 2011 gab es ein Ereignis des Durchsickerns der registrierten Daten beim CSDN (Chinese Software Developer Network). CSDN wurde Opfer eines Hackerangriffs; eine Datenbank mit den Informationen von 6 Millionen bei CSDN registrierten Internetnutzern, einschließlich ihrer Nutzernamen, Passwörter, registrierten Email-adressen, wurden im Internet offengelegt. ${ }^{708}$ Jedoch ist das nur ein kleines Beispiel. Fast alle bekannten großen Webseiten in China wurden bereits Opfer von Hackerangriffen. Es ist deswegen nicht unvorstellbar, dass die Datenbank des Real-Name-System mit allen realen Informationen der Internetnutzer das Ziel von Hackern sein würde, weil diese Informationen ein hohes wirtschaftliches Interesse herbeiführen können. Die Durchsetzung vom Real-Name-System hat ohne Zweifel die persönlichen Informationen der Internetnutzer an das

704 Jiang Zengpei, Dong fang wang, http://www.jxnews.com.cn/jxcomment/system/2008/01/02/002647086.shtml (besucht am 04.04.2015).

705 Song Wei/Tan Jing/Guo Peng, Zheng yi wang, 02.07.2008, http://news.xinhuanet. com/legal/2008-07/02/content_8473380.htm (besucht am 04.04.2015).

706 Zhou Yongkun, Jinan Journal (Philosophy and Social Sciences) 2013, No. 2, 1, 4; siehe auch http://www.cctvxwpd.com/detail.asp?id=6516 (besucht am 04.04.2015).

707 Yang Fuzhong, Studies in Law and Business 2012, No. 5, 32, 33.

708 Qi Aimin, Chinese Social Sciences Today, 02.04.2012, S. A07; siehe auch http:// en.wikipedia.org/wiki/CSDN (besucht am 04.04.2015). 
Messer des Hackers geliefert. ${ }^{709}$ Es ist auch nicht zu vernachlässigen, dass manche Unternehmen aufgrund ihrer wirtschaftlichen Interessen auch am Sammeln und Verkaufen persönlicher Daten interessiert sind, was ebenfalls die Sicherheit der Datenbank gefährdet. ${ }^{710}$

Übrigens ist die Datenbank des Real-Name-System in Bezug auf Undichtigkeiten und Durchsickern der Informationen völlig anders zu betrachten als die Datenbank mit registrierten Informationen der Internetnutzer von einer Webseite. Wenn z.B. die Datenbank von einem Sozialnetzwerk undicht ist, kann man durch einfaches Ändern des Benutzernamens, des Passwortes und der Emailadresse den weiteren Schaden vermeiden. Aber wenn die Datenbank des Real-Name-Systems einmal offengelegt ist, können die persönlichen Daten nicht mehr zurückgezogen werden. Und es ist fast unmöglich, den realen Namen und die ID-Nummer aller Personen zu ändern. Durch die Änderung würden auch riesige Kosten für die Personen und den Staat entstehen. Also ein „Leak“ in der Datenbank des Real-Name-System wäre eine pure Katastrophe.

\section{(3) Real-Name-System ermöglicht den Missbrauch der persönlichen}

Daten durch den Staat

Wie oben bereits gesagt wurde, muss die Datenbank für Real-Name-System stabil genug sein, um vor einem Eingriff durch Hacker geschützt zu sein. Die Datenmenge unter Berücksichtigung der Gesamtbevölkerung Chinas muss übrigens auch dazugerechnet werden. Für die Entwicklung eines solchen Systems kann der normale ISP offensichtlich nicht leisten. ${ }^{711}$ Die Aufgabe muss vom Staat übernommen werden. ${ }^{712}$ Tatsächlich hat die chinesische Regierung auch aktiv diese Aufgabe übernommen. ${ }^{713}$ Das führt dazu, dass der Staat die Informationen, wann wer was im Internet eingetragen hat, sammeln kann. Es ist anzumerken, dass China bisher noch kein Datenschutzgesetz hat. Unter diesem Umstand besteht die hohe Gefahr, dass der Staat die Informationen missbraucht, weil es keine Regelungen gibt, wie der Staat die gesammelten Informationen verwenden soll. Der Missbrauch besteht besonders darin, dass der Staat mittels Real-Name-System das online Verhalten des Volkes überwacht. ${ }^{714}$

709 Vgl. Han, Ning, Legal Science Monthly 2012, No. 4, 3, 7, 9.

710 Zhou Yongkun, Jinan Journal (Philosophy and Social Sciences) 2013, No. 2, 1, 4.

711 Lu Wei, Lanzhou Academic Journal 2012, No. 9, 161, 164.

712 Wang Gang, The Time Weekly, 09.01.2012.

713 http://www.gov.cn/zwgk/2013-03/28/content_2364821.htm_(besucht am 04.04.2015).

714 Zhou Yongkun, Jinan Journal (Philosophy and Social Sciences) 2013, No. 2, 1, 3. 
(4) Real-Name-System ist schädlich für die Erziehung des demokratischen Gedankens und die soziale Sicherheit

Anonymität ist eine grundsätzliche Voraussetzung für die Entwicklung der Demokratie. ${ }^{715}$ Der Politiker hat naturgemäß die Angst, aufgrund seiner politischen Meinung gerächt zu werden. Anonymität kann diese Angst des Politikers am besten vermindern. ${ }^{716}$ Das ist auch der wesentliche Grund, warum eine Abstimmung immer anonym ist. ${ }^{717}$ Das gilt auch für die Demokratie im Internet. Wegen der mangelnden Möglichkeiten zur Teilnahme an der Politik oder anderer staatlichen Angelegenheiten hat das Internet ein Feld geschaffen, um dort den demokratischen Gedanken zu erziehen und um manchmal sogar unmittelbar an der Politik teilzunehmen. ${ }^{718}$ Der Hauptgrund liegt darin, dass die Internetnutzer wegen der Anonymität im Internet ihre Meinungen relativ frei äußern können. Die Durchsetzung des Real-Name-System hat den Weg der demokratischen Entwicklung im Internet gesperrt.

Die anonyme öffentliche Diskussion ermöglicht den Menschen auch, ihre Unzufriedenheit mit der Regierung auszudrücken. Das dadurch entstandene "Ventil" wird vom Volk genutzt, um schlechte Laune abzulassen, was wiederum mehr Sicherheit in die Gesellschaft bringt. Das Blockieren oder die Beschränkung der anonymen öffentlichen Diskussion könnte langsam zu einem Vulkanausbruch ähnlichen Bewegung führen, um eine freie Meinungsäußerung von der Regierung zu erlangen. ${ }^{719}$

(5) Das Vorbild von Südkorea ist als ein Fehler erwiesen

Anonymität im Internet ist das übliche Prinzip auf der Welt. Die meisten Länder haben das Prinzip akzeptiert. Südkorea ist eine Ausnahme, die China immer als Vorbild genommen hat. Aber auch die Praxis in Südkorea hat schon bewiesen, dass das Real-Name-System nicht funktioniert. ${ }^{720}$ Am 23.08.2012 hat das Verfassungsgericht in Südkorea entschieden, dass das Real-Name-System ver-

715 Xu Zhenzeng, Hebei Law Science 2012, No. 9, 166, 167.

716 Xu Zhenzeng, Hebei Law Science 2012, No. 9, 166, 167.

717 Xu Zhenzeng, Hebei Law Science 2012, No. 9, 166, 168; Wang Zhigang, Journal of Beijing University of Aeronautics and Astronautics (Social Sciences Edition) 2006, No. 6, 46, 47f.

718 Xu Zhenzeng, Hebei Law Science 2012, No. 9, 166, 171.

719 Vgl. Yang Fuzhong, Studies in Law and Business 2012, No. 5, 32; Xu Zhenzeng, Hebei Law Science 2012, No. 9, 166, 169; Han, Ning, Legal Science Monthly 2012, No. 4, 3, 9; Zhou Yongkun, Jinan Journal (Philosophy and Social Sciences) 2013, No. 2, 1, 4.

720 Zhou Yongkun, Jinan Journal (Philosophy and Social Sciences) 2013, No. 2, 1, 2. 
fassungswidrig ist. Aufgrund dieser Entscheidung wird das Real-Name-System in 5 Jahren in Südkorea abgeschafft. ${ }^{721}$ Der erfolglose Versuch Südkoreas sollte für China eine Warnung sein. ${ }^{722}$

\section{ee) Zwischenergebnis}

Internetgewalt ist nach allem nur ein kleiner Teil der Internetwelt, die auch durch mildere Wege als Real-Name-System vermindert werden kann. Nur um die Internetgewalt ein bisschen schneller und einfacher zu lösen, aber deswegen eine ganze Reihe von Nachteile hingenommen werden müssen, besonders dass die Meinungsfreiheit des ganzen Volks beschränkt werden muss, ist die Durchführung eines nicht unbedingt funktionierten Real-Name-System nicht verhältnismäßig. ${ }^{723}$

\section{Real-Name-System in Deutschland}

Anonymität und Pseudonymität im Internet ist in Deutschland gesetzlich anerkannt und garantiert. Gemäß $\$ 13$ Abs. 6 TMG hat der Dienstanbieter die Nutzung von Telemedien und ihre Bezahlung anonym oder unter Pseudonym zu ermöglichen, soweit dies technisch möglich und zumutbar ist. Aber im Gegensatz zur überwiegende intensiven Kritik gegen Real-Name-System in China, gibt es in der deutschen Literatur die Tendenz, die Anonymität im Internet einigermaßen zu beschränken.

\section{a) Kein Grundrecht auf Anonymität}

Über die Grundlage der Anonymität gibt es in der Literatur unterschiedliche Meinungen. Zum einen existiert die Meinung, dass es bei der Meinungsäußerung das Grundrecht auf Anonymität gibt, das seine Grundlage in Art. 1 Abs. 1 i.V.m. Art. 2 Abs. 1 sowie Art. 5 Abs. 1, 10 und 13 GG findet. ${ }^{724}$ Zum anderen existiert eine Meinung, die für ein eigenständiges Grundrecht auf Anonymität keinen Bedarf sieht, weil das Recht auf informationelle Selbstbestimmung zum Schutz der Internetnutzer vor Ausspähung ausreicht. ${ }^{725}$ Allerdings stimmt die Meinung für ein Grundrecht auf Anonymität zu, dass das Grundrecht auf Anonymität mit dem Recht auf informationelle Selbstbestimmung korrespondiert,

721 Wikipedia, http://en.wikipedia.org/wiki/Real-name_system (besucht am 04.04.2015).

722 Zhou Yongkun, Jinan Journal (Philosophy and Social Sciences) 2013, No. 2, 1, 7.

723 Han, Ning, Legal Science Monthly 2012, No. 4, 3, 9; Lu Wei, Lanzhou Academic Journal 2012, No. 9, 161, 163.

724 Heckmann, NJW 2012, 2631, 2632.

725 Spindler, GRUR 2013, 996, 1001. 
wonach jeder Internetnutzer selbst entscheiden können soll, wer erfährt, welche Handlungen von ihm stammen. ${ }^{726}$

Meiner Meinung nach ist ein Grundrecht auf Anonymität in Deutschland nicht begründet. $\$ 13$ Abs. 6 TMG hat dem ISP die Möglichkeit gegeben, die Anonymität oder die Pseudonymität für seinen Dienst auszuwählen. Wenn sich der ISP für die Pseudonymität entscheidet, hat der Nutzer keinen Anspruch auf anonymisierte Benutzung des Dienstes, wenn er eigenständig den Dienst des ISP in Anspruch nimmt. Gemäß $\$ 13$ Abs. 6 TMG ist es durchaus möglich und rechtmäßig, dass jeder ISP in Deutschland statt der Anonymität die Pseudonymität für seinen Dienst durchführt, obwohl in der Praxis die Gefahr bestehen könnte, dass er deswegen seine Kunden verliert. Wenn es bei dem Grundrecht auf Anonymität auf die freie Entscheidung des ISP ankommt, ist nicht nachvollziehbar, dass es noch ein Grundrecht ist.

\section{b) Die Einschränkung der Anonymität im Internet}

Nach der aktuellsten Entwicklung in der Literatur ist es schon allgemein anerkannt, dass die Anonymität im Internet eingeschränkt werden soll. ${ }^{727}$ Über den Grad der Einschränkung gibt es jedoch unterschiedliche Meinungen.

Es herrscht die Meinung, dass eine allumfassende anlasslose Identifizierung der Nutzer im Internet zu vermeiden ist. ${ }^{728}$ Dennoch soll der ISP für besonders gefahrgeneigte Dienste verpflichtet sein, die Identität ihrer Nutzer zu verifizieren, ausreichend auch in pseudonymer Form, ${ }^{729}$ um den Auskunftsanspruch im Fall der Rechtsverletzung zu garantieren. ${ }^{730}$

Er herrscht auch die Meinung, dass alle Internetnutzer, die aktiv Informationen im Netz veröffentlichen, dies in pseudonymer Form (oder unter Klarnamen) tun müssen. ${ }^{731}$ Anonymisiert sind dagegen nur die Nutzer, die das Internet passiv als Informationsquelle nutzen.

726 Heckmann, NJW 2012, 2631, 2632.

727 Spindler, GRUR 2013, 996; Herwig, ZD 2012, 558; Dix, Zitat aus: Begleitheft zum Deutschen Juristentag 2012, Thesen der Gutachter \& Regerenten, S. 70 ff, abrufbar unter: http://www.djt.de/fileadmin/downloads/69/120809_djt_69_thesen_web.pdf. (besucht am 04.04.2015).

728 Spindler, GRUR 2013, 996, 1001.

729 Spindler, GRUR 2013, 996, 1001.

730 Spindler, NJW-Beil. 2012, 98, 99.

731 Dix, Zitat aus: Begleitheft zum Deutschen Juristentag 2012, Thesen der Gutachter \& Regerenten, S. 70 ff, abrufbar unter: http://www.djt.de/fileadmin/downloads/69/120809_djt_69_thesen_web.pdf. (besucht am 04.04.2015). 
Es herrscht weiterhin die Meinung, dass der ISP selbst entscheiden soll, ob er die Anonymität oder Pseudonymität für seinen Dienst wählt. Wenn der ISP seinen Dienst für die Nutzer aktiv anonymisiert, kann er theoretisch den Auskunftsanspruch des Verletzten im Fall der Rechtsverletzung nicht mehr erfüllen. ${ }^{732}$ In diesem Fall muss er die anonymisierten Inhalte stärker kontrollieren und für die rechtswidrigen Inhalte als ICP haften. ${ }^{733}$ Wenn der ISP dagegen Pseudonymität von seinen Nutzern verlangt und ggf. den Verletzten über die Identität der rechtsverletzenden Nutzer informieren kann, haftet er im Fall der Rechtsverletzung nur als Störer. ${ }^{734}$

Am Fall der Menschenfleischsuche kann man deutlich sehen, dass nicht nur das Verhalten des Nutzers, der aktiv Informationen veröffentlicht, sondern auch das des Nutzers, der das Internet als Informationsquelle passiv verwendet, Rechtsverletzungen begründen kann. Durch diesen Standard die Anonymität und Pseudonymität zu unterscheiden ist meiner Meinung nach unpraktisch.

Die anderen zwei Meinungen können meiner Meinung nach kombiniert betrachtet werden. Für den besonders gefahrgeneigten Dienst, in dem Rechtsverletzungen häufig auftauchen, ist es für den ISP vernünftiger, von den Nutzern zu verlangen, sich unter einem Pseudonym zu registrieren, bevor sie im Internet aktiv werden. Das Problem liegt nur darin, ob dies vom ISP frei entschieden werden $\mathrm{kann}^{735}$ oder als eine Pflicht durchgeführt wird ${ }^{736}$. Um diese Frage zu beantworten, soll Perspektive des ISP und des möglichen Verletzten betrachtet werden.

Wenn sich der ISP statt für Pseudonymität für Anonymität entscheidet, muss er mehr Pflichten bezüglich der Überwachung der Inhalte auf seiner Webseite haben, um die möglichen Verletzungen zu vermeiden, für die er als unmittelbarer Verletzer (ICP) haften müsste, obwohl er dafür mehr Nutzer bekommen könnte, weil die vernünftigen Nutzer es bevorzugen, anonym zu bleiben. Die Kosten für die allgemeine Überwachung und das Risiko, für die Rechtsverletzung seiner Nutzer unmittelbar zu haften, können schwerwiegender sein. Jedoch darf sich der ISP nach seiner freiwilligen Abwägung auch für die Anonymität entscheiden, weil kein überwiegend zu schützendes Interesse anzunehmen ist. Der Schutz der Verletzten führt auch nicht zu einem Verbot des anonymisierten Dienstes, weil der Verletzte in diesem Fall direkt Ansprüche

732 Herwig, ZD 2012, 558, 560.

733 Herwig, ZD 2012, 558, 562.

734 Herwig, ZD 2012, 558, 560.

735 Herwig, ZD 2012, 558, 562.

736 Spindler, GRUR 2013, 996, 1001. 
gegen den ISP bzw. den ICP geltend machen kann, was für den Verletzten einfacher und praktischer ist.

Nach meiner Meinung soll der ISP nicht gezwungen werden, Pseudonymität für seinen Dienst durchzuführen. Aber die Möglichkeit, im Fall der Rechtsverletzung einen Rechtsanspruch direkt gegen den Provider zu verlangen, soll garantiert werden. Wenn anonymisierte Einträge auf seiner Webseite zur Rechtsverletzung führen, soll der ISP wegen Zueigenmachen verantwortlich sein. ${ }^{737}$ Müsste er ständig deswegen haften, würde er schon automatisch die Pseudonymität seines Diensts bevorzugen.

\section{Ein Vergleich zwischen Deutschland und China}

Obwohl in Deutschland auch versucht wird, die Anonymität zu beschränken und in manchen Fällen die Identität der Internetnutzer zu verifizieren, ist es immer noch anders als das Real-Name-System in China.

Der Unterschied liegt erstens darin, dass die Beschränkungen der Anonymität in Deutschland durch die ISP durchgeführt werden oder werden sollen, während die Durchführung des Real-Name-System in China die Aufgabe der Regierung ist.

Der Unterschied liegt weiterhin darin, dass die Beschränkung der Anonymität in Deutschland rechtsverletzungs-orientiert ist. Nach den Vorschlägen der Juristen ist die Beschränkung nur für besonders gefahrgeneigte Dienste oder aktive Veröffentlichung der Informationen höchst notwendig, wodurch Rechtsverletzungen leicht entstehen. In China wird das Real-Name-System allgemein und umfassend durchgeführt.

Der letzte Unterschied liegt darin, dass es in Deutschland entwickelte und ausführliche Datenschutzgesetze gibt, durch die man den Missbrauch der Daten der Internetnutzer durch den Staat vermeiden kann, während es in China nur eine Informationsschutz-Entscheidung gibt, die 12 schwache Paragraphen beinbehaltet. Bei der Durchführung des Real-Name-System unter diesem Umstand besteht eine hohe Gefahr des Datenmissbrauchs durch den Staat.

Jedoch ist es auch in China zu empfehlen, Pseudonymität für die aktive Teilnahme an der Menschenfleischsuche durchzuführen, um im Fall von Rechtsverletzung die Identifizierung des unmittelbaren Verletzers zu ermöglichen.

737 Siehe oben unter $\$ 9$ I 1. 


\section{$\$ 12$ Rechtsansprüche gegen den ISP als Störer}

\section{Nach deutschem Recht}

\section{Die Reihenfolge der Ansprüche gegen den Internetnutzer als unmittelbarer Verletzer und den ISP als Störer}

Im Jahr 2006 hat das OLG Düsseldorf in seiner Rechtsprechung die Anonymität der rechtswidrigen Einträge als Kriterium für das Bestehen eines Unterlassungsanspruchs gegen den ISP entschieden. ${ }^{738}$ Das bedeutet, der Kläger muss die Reihenfolge seines Anspruchs gegen den unmittelbaren Verletzer und den ISP vor Gericht einhalten. Eine direkte Klage gegen den ISP ist erst möglich, wenn der unmittelbare Verletzer wegen Anonymität unbekannt ist. Das OLG Düsseldorf hat einmal einen gegen den ISP gestellten Unterlassungsanspruch abgelehnt, weil der Autor der rechtswidrigen Inhalte dem Verletzten bekannt war, und der Verletzte somit gegen den Autor vorrangig vorgehen könne. ${ }^{739}$ Umgekehrt hat das Gericht einen Unterlassungsanspruch bejaht, weil der Verletzer anonym war, und der Provider dessen Identität nicht preisgab. ${ }^{740}$

Diese Entscheidungen haben von der Literatur viele Kritiken bekommen. Der BGH hat durch sein Urteil vom 27.03.2007 eine völlig andere Meinung dargelegt. Aufgrund dieser Rechtsprechung gibt es zwischen dem Provider und dem Äußernden in einem Meinungsforum keinen Anspruchsvorrang. ${ }^{741}$ Gegen wen der Betroffenen seinen Anspruch geltend machen soll, „liegt allein im Ermessen des Betroffenen, wie er effizient seine Rechte verfolgt “ ${ }^{742}$ Die zivilrechtliche Verantwortlichkeit des Providers für die auf seiner Webseite eingestellten Beiträge entfällt nicht deshalb, weil dem Verletzten die Identität des Äußernden bekannt ist. ${ }^{743}$

Auch diese Rechtsprechung wurde nicht problemlos akzeptiert. Es herrscht die Meinung, dass das Fehlen des Subsidiaritätsgrundsatzes zwischen den Ansprüchen gegen den Provider und den unmittelbaren Verletzter zur Folge hat, dass der Weg von einer direkten Auseinandersetzung zwischen den Konfliktparteien (der

738 OLG Düsseldorf, CR 2007, 588.

739 OLG Düsseldorf, CR 2007, 588.

740 OLG Düsseldorf, CR 2007, 588.

741 BGH, CR 2007, 586, 589.

742 BGH, CR 2007, 586, 589.

743 BGH, CR 2007, 586, 587. 
Verletzte und der Äußernde) gesperrt worden ist. ${ }^{744}$ Diese Sperrung dient erstens nicht dazu die Rechtsverletzung von der Quelle an zu vermindern; sie führt zweitens dazu, dass sich der ISP als ein „Ersatz“-Richter verhalten muss, wozu er nicht unbedingt in der Lage ist. ${ }^{745}$ Der zweite Aspekt wird im Folgenden diskutiert.

\section{Die Störerhaftung des ISP}

Oben unter $₫ 10$ I 2 sind die Voraussetzungen einer Störerhaftung ausgeführt worden. Sind die Voraussetzungen erfüllt, muss der ISP als Störer haften. Wegen einer Persönlichkeitsrechtsverletzung kommen die Beseitigungs- und Unterlassungsansprüche in Frage, die ihre Grundlage in $₫ 1004$ BGB finden. ${ }^{746}$

\section{a) Die Beseitigungs- und Unterlassungsansprüche fallen nicht unter die Haftungsprivilegierung des $\$ 10$ Satz 1 TMG}

Vor einer ausführlichen Diskussion über Beseitigungs- und Unterlassungsansprüche muss erst die Frage beantwortet werden, ob die Beseitigungs- und Unterlassungsansprüche unter die Haftungsprivilegierung des $\$ 10$ Satz 1 TMG fallen. Wäre die Frage zu bejahen, bräuchte der ISP für den Fall der Unschuld, also Unkenntnis der rechtswidrigen Handlung oder der rechtswidrigen Information, überhaupt nicht haften. Das heißt, auch die Beseitigungs- und Unterlassungsansprüche gegen ihn wären in diesem Fall erfolglos.

Aber der BGH hat durch seine Entscheidung „Internetversteigerung I“ diese Möglichkeit verneint. Seiner Meinung nach erfasst die Haftungsprivilegierung des Art. 14 Abs. 1 RL 2000/31/EG (korrespondiert mit \$ 10 Satz 1 TMG, damals umgesetzt durch $\$ 11$ Satz 1 TDG 2001), nicht die Beseitigungs- und Unterlassungsansprüche. ${ }^{747}$ Der Grundgedanke liegt im Satz 2 des $\$ 11$ TDG n. F., dass die „Verpflichtungen zur Entfernung oder Sperrung der Nutzung von Informationen nach den allgemeinen Gesetzen... auch im Falle der Nichtverantwortlichkeit des Dienstanbieters nach den $\$ \$ 9$ bis 11 unberührt bleiben“. ${ }^{748}$

Meiner Meinung nach kann man im $₫ 10$ TMG selbst die Antwort finden. $\mathrm{Ab}$ dem Zeitpunkt, wenn ein Beseitigungs- oder Unterlassungsanspruch gegen den ISP erhoben wird, erhält der ISP die Kenntnisnahme über die rechtswidrige Handlung oder Information. Es begründet tatsächlich die Kenntnisnahme i.S.v.

744 Spindler, CR 2012, 176, 178.

745 Spindler, CR 2012, 176, 178.

746 Spindler/Anton in Spindler/Schuster, $\$ 1004$ BGB, Rn. 2.

747 Rössel, CR 2011, 589, 590; vgl. auch BGH, CR 2008, 579; BGH, MMR 2007, 507.

748 BGH, GRUR 2004, 860, 863. 
$\$ 10$ Satz 1 Nr. 2 TMG, nach der der ISP die rechtswidrige Handlung oder Information unverzüglich beseitigen muss. Eine Unkenntnis bzw. Unschuld des ISP könnte höchstens bis zum Zeitpunkt existieren, bevor ein Beseitigungs- oder Unterlassungsanspruch gegen ihn gestellt wird. Danach hat er keinen Grund mehr, einen Beseitigungs- oder Unterlassungsanspruch abzuwehren. Eine Privilegierung beider Ansprüche ist demnach sinnlos.

$\$ 1004$ BGB gewährt dem Verletzten einen verschuldensunabhängigen Beseitigungs- und Unterlassungsansprüche gegen den ISP. ${ }^{749}$

\section{b) Beseitigungsanspruch}

Wie bereits ausgeführt worden ist, ist ein ISP nicht verpflichtet, die von seinen Nutzern auf die Webseite gestellten Beiträge vor der Veröffentlichung auf eventuelle Rechtsverletzungen hin zu überprüfen. ${ }^{750}$ Aber sobald er Kenntnis von der Rechtsverletzung erlangt, entsteht die Verantwortlichkeit. ${ }^{751}$ Die Verantwortlichkeit zeigt sich im Fall vom Beseitigungsanspruch als eine Pflicht, die in Frage gestellten Einträge zu löschen oder zu sperren, um die Rechtsverletzung zu beseitigen. Anders als ein Unterlassungsanspruch richtet sich der Beseitigungsanspruch auf die Beendigung der gegenwärtigen Störung. ${ }^{752}$

Der Beseitigungsanspruch bedeutet nach der Rechtsprechung des BGH aber nicht, dass der ISP auf jedes Verlangen des Betroffenen aktiv werden muss, die der Rechtsverletzung verdächtigten Einträge zu löschen. ${ }^{753}$ Sonst würde die Meinungsfreiheit der Äußernden überhaupt nicht geschützt. Durch ein Urteil im Jahr 2011 hat der BGH festgestellt, dass ein Tätigwerden des Providers erst veranlasst wird, wenn die Behauptung des Betroffenen so konkret gefasst ist, dass der Rechtsverstoß ,unschwer - das heiß ohne eigehende rechtliche und tatsächliche Überprüfung- bejaht werden kann" ${ }^{\text {" }}{ }^{75}$ Dieses vom BGH entwickelte Prinzip korrespondiert mit $\$ 10$ Satz 1 Nr. 2 TMG, in dem das auf EU-Ebene gerade heftig diskutierte „Notice-and-Take-Down“-Verfahren vorliegt. ${ }^{755}$

749 Spindler/Anton in Spindler/Schuster, $\$ 1004$ BGB, Rn. 1.

750 BGH, K\&R 2012, 110, 113.

751 BGH, K\&R 2012, 110, 113.

752 Spindler/Anton in Spindler/Schuster, $\$ 1004$ BGB, Rn. 13; BGHZ 28, 110, $113=$ NJW 1958, 1580, 1581; Bassenge in Palandt, $\$ 1004$ BGB Rn. 28; Fritzsche in Bamberger/ Roth, $\$ 1004$ BGB Rn. 56.

753 BGH, K\&R 2012, 110, 113.

754 BGH, K\&R 2012, 110, 113.

755 Hoeren, MMR 2012, 124, 127. 
Das „Notice-and-Take-Down“-Verfahren stammt aus den USA. Aufgrund Section 512 (c) des US-amerikanischen Digital Millenium Copyright Act 1998 (DMCA) muss der Provider den beanstandeten Inhalt nach Eingang einer Beschwerde des Betroffenen sofort sperren. ${ }^{756}$ Im Anschluss muss er dem verdächtigen Verletzer nach Section 512 (g) DMCA Gelegenheit zur Stellungnahme geben. ${ }^{757}$ Erhebt der Betroffene auf diese Stellungnahme hin innerhalb einer Frist von zehn Tagen Klage, bleibt der beanstandete Inhalt gesperrt. ${ }^{758}$ Sieht der Betroffene von einer Klage ab, wird die Sperrung wieder aufgehoben. ${ }^{759}$

Aber statt dem „Notice-and-Take-Down“-Verfahren hat der BGH in diesem Urteil ein sogenanntes „Notice-and-Action“-Verfahren (oder „QuasiNotice-and-Take-Down“-Verfahren ${ }^{760}$ ) entwickelt, ${ }^{761}$ das von der Europäischen Kommission in ihrer Mitteilung zum elektronischen Geschäftsverkehr und zu anderen Online-Diensten von Januar 2012 anerkannt wurde. ${ }^{762}$ Der Unterschied zwischen diesen zwei Verfahren liegt darin, dass das „Notice-and-Action“Verfahren nicht zu einer sofortigen Sperrung der beanstandeten Inhalte, sondern lediglich zu einer Untersuchung des beanstandeten Sachverhalts führt. ${ }^{763}$ Dieses „Notice-and-Action“-Verfahren wird in der Literatur auch als Kommunikationsprozess bezeichnet. ${ }^{764}$

\section{aa) Der Vorgang des Kommunikationsprozesses}

Falls dem Provider unklar ist, ob die Beanstandung des Betroffenen annahmebedürftig ist, ist eine Ermittlung und Bewertung des gesamten Sachverhalts unter Berücksichtigung einer etwaigen Stellungnahme des Äußernden erforderlich. ${ }^{765}$ Der Ermittlungs- bzw. Bewertungsprozess läuft wie folgt ${ }^{766}$ :

756 Rühl, LMK 2012, 338417.

757 Rühl, LMK 2012, 338417.

758 Rühl, LMK 2012, 338417.

759 Rühl, LMK 2012, 338417.

760 Spindler, CR 2012, 176, 178.

761 Rühl, LMK 2012, 338417.

762 Rühl, LMK 2012, 338417.

763 Rühl, LMK 2012, 338417.

764 Feldmann, K\&R 2012, 113, 115.

765 BGH, K\&R 2012, 110, 113.

766 BGH, K\&R 2012, 110, 113. 
(a) Zunächst ist die Beanstandung des Betroffenen an den verdächtigen Verletzer zur Stellungnahme weiterzuleiten.

(b) Bleibt eine Stellungnahme innerhalb einer nach den Umständen angemessenen Frist aus, ist von der Berechtigung der Beanstandung auszugehen und der beanstandete Eintrag zu löschen.

(c) Stellt der verdächtige Verletzer die Berechtigung der Beanstandung substantiiert in Abrede und ergeben sich deshalb berechtigte Zweifel, ist der Provider grundsätzlich gehalten, dem Betroffenen dies mitzuteilen und ggf. Nachweise zu verlangen, aus denen sich die behauptete Rechtverletzung ergibt.

(d) Bleibt eine Stellungnahme des Betroffenen aus oder legt er ggf. erforderliche Nachweise nicht vor, ist eine weitere Prüfung nicht veranlasst.

(e) Ergibt sich aus der Stellungnahme des Betroffenen oder den vorgelegten Belegen auch unter Berücksichtigung einer etwaigen Äußerung des verdächtigen Verletzer eine rechtswidrige Verletzung des Persönlichkeitsrechts, ist der beanstandete Eintrag zu löschen.

Wegen der Erfindungen dieses außergerichtlichen schlichtungsähnlichen Prozesses wurde das Urteil spontan als „legendär“ bezeichnet. ${ }^{767}$ Der Pressesenat des BGH hat mit diesem Prozess ein neues Haftungsmodell für die Internetwelt entwickelt. ${ }^{768}$ Aufgrund der Entscheidung sind nicht nur Verhaltenspflichten für den Provider, sondern auch die für den Betroffenen und den Äußernden beim Streitfall eingerichtet. ${ }^{769}$

Der Provider soll den Kommunikationsprozess zwischen den Betroffenen und den Äußernden durchführen. Darauf sollen der Betroffene und der Äußernde demgemäß reagieren. Der Betroffene muss nicht nur die Tatsache der Veröffentlichung eines rechtswidrige Drittinhaltes dem Provider mitteilen, sondern auch begründen, aus welchen Gründen die Information rechtswidrig ist. ${ }^{770} \mathrm{Dem}$ entsprechend muss sich der Äußernde für seine Äußerung verteidigen. Für den Streitfall muss er sein berechtigtes Interesse begründen. Zum Schluss soll der Provider entweder dem Äußernden oder dem Betroffenen den Vorzug geben. ${ }^{771}$ Der Provider ist „nicht lediglich Moderator und Vermittler dieses Kommunikationsprozesses“, „sondern eine Art Schiedsrichter in einer Auseinandersetzung“.772

767 Feldmann, K\&R 2012, 113, 114.

768 Hoeren, MMR 2012, 124, 127.

769 Feldmann, K\&R 2012, 113, 114.

770 Feldmann, K\&R 2012, 113, 114.

771 Feldmann, K\&R 2012, 113, 115.

772 Feldmann, K\&R 2012, 113, 115. 
Dieser Kommunikationsprozess ist die aktuellste Methode, um den Beseitigungsanspruch im Fall einer Persönlichkeitsrechtsverletzung während der Menschenfleischsuche durchzuführen. Seiner Anwendung stehen jedoch viele Kritiken gegenüber.

\section{bb) Die Kommentare über den Kommunikationsprozess}

(1) Die Gefahr der extra entstehenden Pflichten und Kosten des ISP

Um die Aufgabe des ISP im Kommunikationsprozess zu beschreiben, hat der BGH einige unbestimmbare Ausdrücke verwendet, wie z.B. „substantiierte“ Gegenäußerung, „berechtigte Zweifel ${ }^{\text {“773 }}$. Solche Ausdrücke sind relativ subjektiv und umfangreich, die von jedem unterschiedlich beurteilt werden könnten. ${ }^{774}$ Um den Kommunikationsprozess durchzuführen ist eine Prüfung der zu beanstandenden Inhalte vom ISP unvermeidlich. ${ }^{775}$ Wegen der Unbestimmbarkeit des Beurteilungsstandards ist es in der Praxis für den ISP häufig schwer einzuschätzen ${ }^{776}$, ob eine Rechtsverletzung vorliegt. Hierdurch würden den Provider komplexe Prüfungspflichten treffen. ${ }^{777}$

Die Prüfungspflicht während des Kommunikationsprozesses kann nicht durch Computerprogramme automatisiert werden. Um die komplexe Anforderung des BGH zu erfüllen, muss der ISP während des Betriebs der Webseite passende beschwerdebezügliche Verfahren entwickeln. ${ }^{778}$ „Dazu gehört möglicherweise die Entwicklung spezieller Formulare, die Einrichtung von Beschwerdestellen und die Einstellung neuer Mitarbeiter “" ${ }^{\text {779 }}$ Dies bedeutet für den Provider eine deutliche Mehrbelastung und eine enorme Kostensteigerung. ${ }^{780}$

Jedoch hat der BGH in der Entscheidung "Jugendgefährdende Medien bei eBay“ ausdrücklich klargestellt, dass „keine Anforderungen auferlegt werden (dürfen), die (ein) von der Rechtsordnung gebilligtes Geschäftsmodell gefährden oder (die) Tätigkeit unverhältnismäßig erschweren“. ${ }^{\text {(781 }}$

\footnotetext{
773 BGH, K\&R 2012, 110, 113.

774 Vgl. Hoeren, MMR 2012, 124, 127.

775 Rühl, LMK 2012, 338417.

776 Spindler, CR 2012, 176, 178.

777 Hoeren, MMR 2012, 124, 127.

778 Rühl, LMK 2012, 338417.

779 Rühl, LMK 2012, 338417.

780 Rühl, LMK 2012, 338417.

781 BGH, K\&R 2007, 517 ff.
} 
Ob die Pflicht des ISP im Kommunikationsprozess sein Geschäftsmodell gefährdet oder seine Tätigkeit unverhältnismäßig erschwert, hängt mindestens davon ab, wie oft die Rechtsverletzungen passieren und wie oft die Betroffenen diese beanstanden. Weil der Kommunikationsprozess in der Praxis noch nicht umfangreich durchgesetzt ist, kann man diese Frage nicht überzeugend beantworten. Klar ist aber, dass die Anzahl der von den Internetnutzern begangenen Verletzungen hier ein entscheidendes Element ist. Um den Kommunikationsprozess vernünftig durchzusetzen, und gleichzeitig das Geschäftsmodell des ISP nicht zu gefährden oder seine Tätigkeit nicht unverhältnismäßig zu erschweren, ist ein Mechanismus zu überlegen, der die Rechtsverletzungen von der Quelle an vermindert.

(2) Die Gefahr einer haftungsrechtlichen Zwickmühle für den ISP

Der BGH hat bei der Erfindung des Kommunikationsprozesses die Situation vernachlässigt, in der sich eine Persönlichkeitsrechtsverletzung weder zweifelsfrei feststellen noch zweifelsfrei ausschließen lässt ${ }^{782}$. Bei dem Tätigwerden soll es nach den Umständen klar sein, dass ein Rechtsverstoß unschwer bejaht werden kann. Aber nach den gegenseitigen Stellungnahmen des Äußernden und des Betroffenen könnten die Umstände so geändert werden, dass ein Rechtverstoß nicht mehr unschwer bejaht werden kann.

Für diesen Fall erfasst die herrschende Meinung, dass der BGH den Provider von seiner eigentlich neutralen Rolle zu einer haftungsrechtlichen Zwickmühle - Haftung wegen Persönlichkeitsrechtsverletzung einerseits, Haftung wegen Vertragsverletzung andererseits - geführt hat. ${ }^{783}$ Das heißt, für den Fall, in dem der ISP nicht leicht entscheiden kann, muss der ISP das Risiko der Fehleinschätzung selbst tragen ${ }^{784}$; entweder begründet er zugunsten des Betroffenen die Rechtsverletzung und verletzt die vertragliche Pflicht zu seinem Kunden oder er verletzt als Störer das Persönlichkeitsrecht des Betroffenen.

Meiner Meinung nach würde die Zwickmühlenwirkung aus folgenden Gründen überhaupt nicht eintreten:

1. In der Rechtsprechung über den Kommunikationsprozess hat der BGH nicht ausdrücklich festgelegt, dass eine eindeutige Entscheidung zugunsten einer Partei getroffen werden muss. Es wurde zwar geschrieben, dass die Durchführung des Kommunikationsprozesses eine Pflicht der Provider ist; aber diese

782 Rühl, LMK 2012, 338417.

783 Rühl, LMK 2012, 338417.

784 Feldmann, K\&R 2012, 113, 115. 
Pflicht bezieht sich nicht spezifisch oder nicht allein auf den Kommunikationsprozess, sondern auch auf das Tätigwerden des Providers überhaupt. Die Pflicht entsteht, wenn der Provider tätig sein muss; dies setzt voraus, dass der Hinweis des Betroffenen über eine Rechtsverletzung so konkret gefasst ist, dass der Rechtsverstoß auf der Grundlage der Behauptungen des Betroffenen unschwer das heiß ohne eingehende rechtliche und tatsächliche Überprüfung- bejaht werden kann. ${ }^{785}$ Wenn der Provider nach dem Tätigwerden Schwierigkeit mit einer Entscheidung des Rechtsverstoßes hat, bedeutet es dann, dass die Voraussetzung für das Tätigwerden des Providers nicht mehr besteht. Wäre dieses Ergebnis vorauszusehen, sollte der Kommunikationsprozess überhaupt nicht stattfinden. In diesem Fall braucht der Provider meiner Meinung nach nicht unbedingt eine Entscheidung treffen, sondern dem Betroffenen Bescheid geben, sich an ein Gericht zu wenden, um sein Recht zu verteidigen.

2. Auch wenn der Provider eindeutig entscheiden muss, soll er nicht für die falsche Entscheidung das Risiko tragen. Die Durchführung des Kommunikationsprozesses ist eine Pflicht, die der BGH dem Provider erteilt hat. Aber der Provider ist kein professioneller Richter oder Schiedsrichter; von ihm kann nur die zivilrechtliche verkehrsübliche Sorgfalt erwartet werden. ${ }^{786}$ Das bedeutet, dass seine Entscheidung von der Entscheidung des Gerichts abweichen darf. Selbst wenn die falsche Entscheidung von ihm getroffen wird, sollte er nicht deswegen haften, solange er die verkehrsübliche Sorgfalt beachtet und bei der Beurteilung keinen offensichtlichen Fehler gemacht hat.

(3) Die Gefahr der Beschränkung der Meinungsfreiheit

Die Rechtsprechung hat dem ISP eine Rolle eines Richters gegeben. Dem entsprechend erwartet der BGH von dem ISP, idealerweise nach verkehrsüblichem Standard seine Rolle durchzuführen. Aber der ISP ist nichts anders als ein „Homo oeconomicus“, der eigeninteressiert und rational handelt bzw. seinen eigenen Nutzen maximiert. ${ }^{787}$

Nach der Rechtsprechung verlangt das Anschalten des Kommunikationsprozesses die positive Kenntnis der Rechtverletzung. Wenn der Betroffene durch das Beanstanden die Rechtsverletzung gegen ihn nicht deutlich begründen kann, braucht der ISP den Kommunikationsprozess nicht anschalten.

785 BGH, K\&R 2012, 110, 113.

786 Spindler, CR 2012, 176, 178.

787 Franz, http://potsdamer-koepfe.de/u/makrooekonomie/docs/studoc/stud7.pdf. (besucht am 04.04.2015), S. 4. 
Unter dieser Konstellation verletzt der ISP auch keine Prüfungspflicht. ${ }^{788}$ Aber wie es zu beurteilen ist, ob der Betroffenen die Rechtsverletzung hinreichend begründet hat, ist ungewiss. ${ }^{789} \mathrm{Um}$ jeden möglichen Fehler zu beseitigen bzw. eine Haftung von der Seite der Betroffenen zu vermeiden, wird ein umsichtiger Provider aus Sicherheitserwägungen jedes Mal den Kommunikationsprozess anschalten, „selbst wenn die Beanstandung des Betroffenen jedweder fundierten Grundlage entbehrt“ ${ }^{790}$ Dadurch könnten übrigens die Kosten des ISP bei der Beurteilung des Tätigwerdens eingespart werden, weil er nur am Schluss des Kommunikationsprozesses einmalig beurteilen müsse. Dies erhöht entsprechend die Kosten, um Meinungsfreiheit auszuüben, weil der Äußernde auch für seine rechtmäßige Äußerung jeder Zeit bereit sein muss, sich gegen die unvernünftige Beanstandung des Betroffenen zu verteidigen, weil die Äußerung gelöscht werden könnte, wenn er nicht rechtzeitig seine Stellungnahme abgibt.

Das Verhalten des Providers als „Homo oeconomicus" könnte auch bei der Schlussphase passieren. Wie bereits erwähnt wurde, ist der Kommunikationsprozess für den ISP aufwendig. Um die Kosten der Prüfung zu sparen und eine Haftung als Störer zu vermeiden, könnte ein vorsichtiger und vernünftiger Provider ohne Prüfung gegenseitiger Stellungnahmen direkt die bezüglichen Einträge löschen, auch wenn die Gegendarstellung des Äußernden vielleicht haltbar ist und die Entfernung damit eine Verletzung des Vertrags mit dem Nutzer sein könnte. ${ }^{791}$ Ein derartiges Ergebnis wäre sicherlich eine Katastrophe für die Ausübung der Meinungsfreiheit. ${ }^{792}$

Die Beschränkung der Meinungsfreiheit könnte übrigens noch darin liegen, dass der BGH überfordert, dass die beanstandeten Einträge endgültig gelöscht werden sollen, wenn der Äußernde sie nicht erfolgreich als rechtmäßig nachweisen kann. Es herrscht die Meinung, dass eine Sperrung der Einträge, wie es in $\$ 10$ Satz 1 Nr. 2 TMG und auch im „Notice-ande-Take-Down“-Verfahren in den USA geregelt wurde ${ }^{793}$ schon genügt, um eine weitere Persönlichkeitsrechtsverletzung zu verhindern. ${ }^{794}$

788 Feldmann, K\&R 2012, 113, 115.

789 Feldmann, K\&R 2012, 113, 115.

790 Feldmann, K\&R 2012, 113, 115.

791 Feldmann, K\&R 2012, 113, 115.

792 Feldmann, K\&R 2012, 113, 115.

793 Rühl, LMK 2012, 338417.

794 Spindler, CR 2012, 176, 178. 
(4) Die Gefahr des ungenügenden Schutzes im Fall offensichtlicher Rechtsverletzung und der besonders schweren Persönlichkeitsverletzung

Durch diese Rechtsprechung hat der BGH zwei Begriffe aus $₫ 10$ Satz 1 Nr. 2 TMG einen Schritt weiter ausgelegt:

Der Erste Begriff ist die „Kenntnis“. Für den Provider reicht eine bloße Kenntnis durch die Beanstandung des Betroffenen nach der Rechtsprechung nicht mehr, um die Prüfungspflicht des Providers auszulösen. ${ }^{795}$ Dafür muss er eine positive Kenntnis an der Rechtsverletzung eines Dritten haben ${ }^{796}$. Positive Kenntnis bedeutet, dem Provider müssen nicht nur die Tatsachen sondern auch die Umstände bekannt sein, die die Rechtswidrigkeit der Informationen begründen. ${ }^{797}$

Der Zweite Begriff ist „unverzüglich“. Wegen der Änderung der Bedeutung der Kenntnis ist der Begriff „unverzüglich“ dementsprechend auch geändert, weil der Provider den Kommunikationsprozess durchführen muss, um eine positive Kenntnis zu erlangen. Deswegen ist es für den Provider nicht mehr notwendig, den beanstandeten Inhalt nach der Ermittlung des Betroffenen sofort zu entfernen, sondern nur unverzüglich seinen Nutzer zur Stellungnahme aufzufordern bzw. den Kommunikationsprozess anzuschalten. ${ }^{798}$

Diese Änderungen führen direkt dazu, dass der Verletzte auch im Fall der offensichtlichen Rechtsverletzung keinen Anspruch mehr auf sofortige Entfernung der bezüglichen Einträge hat, weil genau die Offensichtlichkeit einer Rechtsverletzung Voraussetzung für das Anschalten des Kommunikationsprozesses ist. ${ }^{799}$

Die Rechtsprechung hat auch keine Sonderregel für den Fall der besonders schweren Persönlichkeitsverletzung geschaffen, die dringend Rechtsschutz benötigt ${ }^{800}$ In diesem Fall könnte die hohe Vermehrungsgeschwindigkeit der Information im Internet dem Betroffenen eine enorme Prangerwirkung bringen, während eine vorläufige Sperrung der Einträge des Äußernden ihm nicht so viel Schaden bringen würde. Übrigens werden die Einträge schnell wieder veröffentlicht, solange der Äußernde seine Stellungnahme durch Belegen rechtzeitig nachweisen kann. Abzuwägen ist in diesem Fall auf einer Seite die Bedrohung

795 OLG Hamburg, K\&R 2006, 470 ff.

796 Feldmann, K\&R 2012, 113, 114.

797 Feldmann, K\&R 2012, 113, 114.

798 Feldmann, K\&R 2012, 113, 114.

799 Hoeren, MMR 2012, 124, 127.

800 Vgl. Hoeren, MMR 2012, 124, 127. 
einer schweren Persönlichkeitsverletzung mit keiner Möglichkeit der Wiedergutmachung und auf der anderen Seite eine vorläufige Beschränkung der Meinungsfreiheit mit leichtem Schaden. Meiner Meinung nach ist der Betroffene schutzwürdiger. Die bezüglichen Einträge sollen in diesem Fall nach der Beanstandung des Betroffenen sofort vorläufig gesperrt werden. ${ }^{801}$

Als eine Unterstützung meiner Meinung hatte das KG Köln in einer Entscheidung über ein Hotelbewertungsportal im Internet für die Seite der Bewertenden entschieden, dass der Portalbetreiber im Fall einer Beschwerde den bewerteten Touristikunternehmen die Möglichkeit geben soll, die beanstandete Äußerung solange nicht online zu stellen, bis deren Berechtigung geklärt ist. ${ }^{802}$ Zum Schutz der Ehre eines Touristikunternehmens ist eine vorläufige Beschränkung der Meinungsfreiheit sogar möglich, ganz zu schweigen vom Schutz des hochwertigeren Persönlichkeitsrechts.

Ein Anschalten des Kommunikationsprozesses ohne Differenzierung der Situation ist also bedenklich. ${ }^{803}$

(5) Der Kommunikationsprozess erfordert den Verzicht der Anonymität

Der Kommunikationsprozess korrespondiert mit der Tendenz, auf die Anonymität für die aktive Teilnahme an der Aktivität im Internet zu verzichten und stattdessen die Pseudonymität durchzuführen. ${ }^{804}$ Um die Durchsetzung des Prozesses zu ermöglichen, müssen erstens die Einträge dem zuständigen Internetnutzer zugeordnet und zweitens die Internetnutzer erreicht werden können. ${ }^{805}$ Dies setzt meiner Meinung nach voraus, dass die Internetnutzer mindestens mit dem Pseudonym registriert sein müssen, bevor sie Einträge auf der Webseite veröffentlichen. ${ }^{806}$

(6) Zusammenfassung über den Kommunikationsprozess

Obwohl der Kommunikationsprozess viele Kritiken bekommen hat, ist er zurzeit die beste Methode, um die Persönlichkeitsrechte des Betroffenen vor weiteren Rechtsverletzungen im Internet rechtzeitig zu schützen. Der ISP ist wegen

\footnotetext{
801 Vgl. Rühl, LMK 2012, 338417.

802 KG Berlin, K\&R 2011, 671, 672.

803 Vgl. Rühl, LMK 2012, 338417.

804 Siehe oben unter $\$ 11$ III 2.

805 Vgl. Rühl, LMK 2012, 338417; Feldmann, K\&R 2012, 113, 115.

806 Vgl. Rühl, LMK 2012, 338417; Hoeren, MMR 2012, 124, 127.
} 
seiner Fähigkeit unfreiwillig an die Stelle wie ein Richter gestellt worden. Von ihm kann man jedoch nur die vernünftige Entscheidung wie von einer durchschnittlichen verständigen Person erwarten, wenn er zwingend eine Entscheidung treffen muss.

Die Rechtsprechung des BGH soll trotzdem so ausgelegt werden, dass der ISP nur im Fall offensichtlicher Rechtsverletzung eine Entscheidung treffen muss. Dies wäre der Fall, wenn der ISP den Rechtsverstoß auf der Grundlage der Behauptungen des Betroffenen unschwer - das heißt ohne eingehende rechtliche und tatsächliche Überprüfung- bejaht werden kann. Wenn sich diese Situation nach der gegenseitigen Stellungnahme des Äußernden geändert hat, das heißt, wenn der Rechtsverstoß nicht mehr eindeutig ist, muss der ISP nicht mehr eine eindeutige Entscheidung zugunsten einer Partei machen, sondern nur den Parteien mitteilen, sich wegen der Schwierigkeit des Falles an das Gericht zu wenden. Wenn der ISP in diesem Fall trotzdem eine Entscheidung getroffen hat, muss er nicht für seine falsche Entscheidung haften, solange er die Sorgfaltspflicht wie eine durchschnittliche verständige Person nicht verletzt hat.

Dies würde nicht dazu führen, dass der ISP eine Entscheidung auch für den Fall der offensichtlichen Rechtsverletzung verweigert, weil er in diesem Fall wegen der Verletzung der Beseitigungspflicht haften muss, wenn das Gericht die Rechtsverletzung nachher als offensichtlich beurteilen würde.

Wenn es in einem komplexen Fall um schwerwiegende Persönlichkeitsrechtsverletzung geht, soll der ISP trotz der Komplexität des Falles eine Entscheidung zugunsten des Betroffenen treffen. Dies wäre z.B. der Fall, wenn durch die Einträge des Äußernden die Intimsphäre einer normalen Person offengelegt wird.

\section{c) Unterlassungsanspruch}

Wie bereits erwähnt wurde, ist ein Beseitigungsanspruch auf die Beendigung der gegenwärtigen Störung gerichtet ${ }^{807}$. Im Gegenteil ist der Unterlassungsanspruch auf die Abwehr erneuter Verletzungen in der Zukunft gerichtet. ${ }^{808}$ Ist eine gleichartige Verletzung in der Zukunft zu erwarten, reicht ein "Take-Down“ allein nicht aus, um den Provider von weiteren Verpflichtungen gegenüber dem Rechtsinhaber freizustellen. ${ }^{809}$ Der Provider hat vielmehr aktiv einer wiederholten bzw.

807 Spindler/Anton in Spindler/Schuster, $\$ 1004$ BGB, Rn. 13; BGHZ 28, 110, $113=$ NJW 1958, 1580, 1581; Bassenge in Palandt, $\$ 1004$ BGB Rn. 28; Fritzsche in Bamberger/ Roth, $\$ 1004$ BGB Rn. 56.

808 Spindler/Anton in Spindler/Schuster, $\$ 1004$ BGB, Rn. 14.

809 Rössel, CR 2011, 593. 
weiteren Rechtsverletzung entgegenzuwirken. ${ }^{810}$ Das ist das sogenannte „Noticeand-Stay-Down“- oder „ Notice-and-Keep-Off“-Verfahren. ${ }^{811}$ Dieses Prinzip hat der BGH in der Entscheidung „Internetversteigerung I“ im Jahr 2004 für Markenrechtverletzungen entwickelt. ${ }^{812}$ Durch die "Blogspot“-Entscheidung von 2011 hat der BGH weiterhin festgestellt, dass es auch auf Persönlichkeitsverletzungen im Internetforum anwendbar ist. ${ }^{813}$

Der Gedanke des Unterlassungsanspruchs findet seine Grundlage auch auf europäischer Ebene. Der EuGH leitet aus dem Zweck der RL 2004/48/EG, effektiven Rechtsschutz zu bieten, sowie deren Erwägungsgrund $25 \mathrm{ab}$, dass aus deren Art. 11 Satz 3 die Verpflichtung der Mitgliedstaaten folgt, gerichtliche Anordnungen gegenüber Mittelspersonen nicht nur zur Beseitigung einer Rechtsverletzung, sondern auch zu deren künftiger Verhinderung vorzusehen. ${ }^{814}$

Im Folgenden werden die Voraussetzungen eines Unterlassungsanspruchs diskutiert.

aa) Die Voraussetzungen eines Unterlassungsanspruchs gegen den ISP im Fall der Persönlichkeitsverletzung

(1) Wiederholungsgefahr und Erstbegehungsgefahr

Ein Störer kann nach der Rechtsprechung nicht nur aufgrund von Wiederholungsgefahr sondern auch wegen Erstbegehungsgefahr ${ }^{815}$ auf Unterlassung in Anspruch genommen werden.

Der Hintergedanke liegt darin, dass der Betroffene bei einer drohenden Gefährdung nicht erst abzuwarten braucht, bis der erste Eingriff in ein Rechtsgut erfolgt ist. ${ }^{816} \mathrm{Um}$ einen solchen Unterlassungsanspruch geltend $\mathrm{zu}$ machen, muss eine Erstbegehrungsgefahr begründet werden.${ }^{817}$ Dies wäre der Fall, wenn es noch nicht zu einer Verletzung des geschützten Rechts gekommen ist, eine Verletzung in der Zukunft aber auf Grund der Umstände zu befürchten ist. ${ }^{818}$

Die Begründung des Unterlassungsanspruchs wegen Erstbegehungsgefahr hat viele Kritiken erhalten, weil es zu einer allgemeinen Überwachungspflicht

810 BGH, GRUR 2004, 860, 862; vgl. auch BGH, K\&R 2012, 110, 113.

811 Rössel, CR 2011, 593.

812 BGH, GRUR 2004, 860, 862; vgl. auch BGH, K\&R 2012, 110, 113.

813 BGH, K\&R 2012, 110, 113.

814 Rössel, CR 2011, 589, 590; EuGH, CR 2011, 597.

815 BGH, MMR 2007, $507 \mathrm{ff}$.

816 Vgl. OLG Hamburg, MMR 2006, 744, 746; s. a. BGH, MMR 2007, 507, 510.

817 BGH, MMR 2007, 507.

818 BGH, MMR 2007, 507. 
des Providers führen könnte, die mit Art. 15 der E-Commerce-Richtlinie nicht zu vereinbaren wäre. ${ }^{819}$

Für den Unterlassungsanspruch nach einer bereits erfolgten Rechtsverletzung kann der Provider aufgrund der Kerntheorie ${ }^{820}$ auf „klare“ und „gleichgelagerte" Rechtsverletzungen aufpassen, die noch als spezifische Überwachungspflicht eingeordnet werden kann. ${ }^{821}$ Für den Fall, das eine Rechtsverletzung nur droht und nicht einmal wirklich passiert, existiert weder ein Inhalt auf dem Server des Providers, noch kann der Provider von dem Inhalt vor seiner Speicherung Kenntnis erhalten. ${ }^{822}$ Um die erste Begehung zu vermeiden, muss der Provider jeden einzelnen neu eingestellten Beitrag vor der Freischaltung prüfen. ${ }^{823}$ Dies könnte zu einer allgemeinen Überwachungspflicht des Providers führen.

$\mathrm{Zu}$ berücksichtigen ist es jedoch, dass die Anerkennung der Erstbegehungsgefahr als Voraussetzung des Unterlassungsanspruchs von dem BGH in einem Spezialfall festgestellt ist. Unter jenen Konstellationen war es möglich, eine bestimmte Rechtsverletzung zu identifizieren oder zu erwarten. Die Voraussetzung für das Vorliegen einer Erstbegehungsgefahr soll deswegen eng gefasst werden. ${ }^{824}$ Die Einwirkung zu einer allgemeinen Überwachungspflicht des Providers ist auf jeden Fall zu vermeiden.

\section{(2) Qualifiziertes Notice}

Ob es nötig ist, eine Rechtsverletzung dem ISP zu beweisen, oder ob es reicht, den ISP auf die vermutliche Rechtsverletzung einfach hinzuweisen, um „Notice-andStay-Down“- oder „ Notice-and-Keep-Off“-Verfahren anzuschalten, hatten das OLG Düsseldorf als Vorinstanz und der BGH in der „Stiftparfüm“-Entscheidung unterschiedliche Meinungen gehabt. ${ }^{825}$

Nach der Meinung vom OLG Düsseldorf reicht die bloße Mitteilung über die Rechtsverletzung nicht, um eine zukünftige Prüfungspflicht des Plattformbetreibers auszulösen. ${ }^{826}$ Vielmehr sei es hierfür erforderlich, Belege für diese Behauptung beizubringen. ${ }^{827}$

819 Vgl. Roggenkamp, jurisPR-ITR 11/2007 Anm. 2.

820 Siehe unten.

821 Roggenkamp, jurisPR-ITR 11/2007 Anm. 2.

822 Vgl. Spindler, MMR 2007, 511, 512.

823 Roggenkamp, jurisPR-ITR 11/2007 Anm. 2.

824 Vgl. Jürgens, K\&R 2007, 392, 393.

825 BGH, K\&R 2011, 727, 729; GRUR 2011, 1038.

826 Volkmann, K\&R 2012, 381, 382.

827 Volkmann, K\&R 2012, 381, 382. 
Der BGH hat die Meinung des OLG Düsseldorf korrigiert und meinte, dass ein Beleg der im Hinweis mitgeteilten Umstände nur dann erforderlich sei, wenn schutzwürdige Interessen des Plattformbetreibers dies rechtfertigten. ${ }^{828}$ Dies könne z. B. der Fall sein, wenn es berechtigte Zweifel am Bestehen des Schutzrechts gibt und die begründete Verletzung vom Provider aufwendige eigene Recherchen verlangen würde. ${ }^{829}$

\section{(3) Zumutbarkeit}

Um eine zukünftig gleichartige Rechtverletzung zu verhindern, ist vom Störer nur eine zumutbare Kontrolle erforderlich. ${ }^{830}$ Es wäre eine Überforderung und würde deswegen zu einer allgemeinen Überwachung führen, wenn der Provider „jeden in einem automatisierten Verfahren von Benutzer unmittelbar ins Internet gestellten Inhalt darauf überprüfen“ muss, um zu sichern, dass keine Schutzrechte Dritter verletzt werden. ${ }^{831}$

Die Standards zur Beurteilung der Zumutbarkeit haben der BGH und der EuGH aus ihren Markenrechtsverletzungs-Entscheidungen entwickelt. Nach der Rechtsprechung des BGH können sie aber auch auf den Fall der Persönlichkeitsrechtsverletzung angewendet werden. ${ }^{832}$

Die Zumutbarkeit soll in jedem einzelnen Fall unter einzelnen Umständen bestimmt werden. Das Hauptprinzip, dass die unternehmerische Freiheit des ISP nicht einschränkt werden soll, soll aber unberührt bleiben. ${ }^{833}$ Übrigens wurden in der Praxis noch andere Prinzipien für die Beurteilung der Zumutbarkeit entwickelt:

(a) Dass der Provider zusätzliches Personal für die Kontrolle einsetzen müsste, ist kein hinreichender Grund einer unzumutbaren Maßnahme. ${ }^{834}$ Das Einsetzen des zusätzlichen Personals ist erst unzumutbar, wenn durch den dadurch entstehenden Überprüfungsaufwand das Geschäftsmodell in Frage gestellt würde. ${ }^{835}$

828 Volkmann, K\&R 2012, 381 382; vgl. BGH, K\&R 2011, 727, 729 = GRUR 2011, 1038.

829 Volkmann, K\&R 2012, 381382.

830 BGH, GRUR 2004, 860, 863; Rössel, CR 2011, 589, 594.

831 BGH, GRUR 2004, 860, 863f.

832 BGH, K\&R 2012, 110, 113 = GRURInt 2012, 259.

833 EuGH, K\&R 2012, 35ff.

834 Spindler/Anton in Spindler/Schuster, § 1004 BGB, Rn. 9; OLG Köln, ZUM 2007, 927, 930.

835 Spindler/Anton in Spindler/Schuster, $\$ 1004$ BGB, Rn. 9; BGHZ 173, 188, 202, Rn. 39 = CR 2007, 728, 732 = MMR 2007, 634, 637; BGH, NJW 2010, 2061, Rn. 24. 
(b) Ist zum Überprüfen der behaupteten Rechtsverletzungen eine uneingeschränkte manuelle Kontrolle der Inhalte erforderlich, ist es dem Provider nicht mehr zumutbar. ${ }^{836}$ Dies wäre nach der Rechtsprechung des BGH der Fall, wenn die zukünftig zu vermeidende Rechtsverletzung keine Merkmale aufweist, die sich zur Eingabe in ein automatisiertes Suchsystem eignen. ${ }^{837}$

(c) Die Zumutbarkeit bzw. die Intensität der Prüfungspflicht kommt auch auf das Geschäftsmodell an. Dafür müssen einige Frage beantwortet werden: Erstens, ob das Geschäftsmodell auf der Nutzung der Rechtswidrigkeit eingestellter Inhalte beruht ${ }^{838}$; zweitens, ob und in welcher Weise der Provider direkt oder indirekt an den rechtswidrigen Inhalten mitverdient ${ }^{839}$; drittens, inwiefern eine rechtswidrige Nutzung der Webseite allgemein bekannt ist $t^{840}$. Je mehr es sich bei dem Provider um rechtswidrige Inhalte handelt, desto intensiver ist seine Prüfungspflicht der fremden Informationen auf seiner Webseite.

\section{bb) Kerntheorie}

Wenn ein Unterlassungsanspruch gegen den ISP begründet ist, ist die Kerntheorie anwendbar.

Auf Grund dieser Theorie verpflichtet sich der ISP nicht nur zur Verhinderung weiterer Verletzungen durch denselben Verletzer, sondern auch die durch andere Internetnutzer begangenen Verletzungen über dieselbe bzw. im Kern gleichen Inhalte. ${ }^{841}$

\section{cc) Selbstaufspürung der Rechtsverletzung durch den Betroffenen}

Die Verurteilung des ISP zur Unterlassungspflicht ist nicht selbstverständlich, weil unter der Situation, wenn weder der Provider noch der Betroffene für die Verletzung schuldig ist, immer die Frage steht, wer von den Beiden unter Effizienzgesichtspunkten derjenige ist, der die Rechtsverletzungen am besten überwachen und verfolgen kann. ${ }^{842}$ Wenn die Überwachung- und Verfolgungspflicht vom ISP an den Betroffenen übertragen werden kann, soll sich der Betroffene

\footnotetext{
836 Volkmann, K\&R 2012, 381 383; vgl. BGH, K\&R 2011, 117 ff.

837 BGH, K\&R 2007, 387 ff. = MMR 2007, 507, 511.

838 Wilmer, NJW 2008, 1845, 1847.

839 Vgl. OLG Düsseldorf, MMR 2006,618; siehe auch Wilmer, NJW 2008, 1845, 1847.

840 Wilmer, NJW 2008, 1845, 1847.

841 Vgl. Rössel, CR 2011, 596; BGH, CR 2007, 728.

842 Spindler, MMR 2007, 507, 514.
} 
mit dem gleichen Aufwand mehr um sich kümmern. ${ }^{843}$ Dieser Gedanke ist von der Rechtsprechung nachgewiesen.

Der BGH hat in der Kinderhochstühle-Entscheidung festgestellt, dass, wenn der ISP (eBay in diesem Fall) ein passendes Programm (VeRI-Programm von eBay) anbietet, mit dem der Betroffenen eine Rechtsverletzung selbst aufspüren kann, die Prüfungspflicht seitens des ISP entfällt. ${ }^{84}$

Der BGH hat mit diesem Urteil der Praxis einen wichtigen Hinweis gegeben, wie „ausufernde Unterlassungspflichten in Zukunft zwar nicht uneingeschränkt aber doch auf bestimmten Ebenen vermieden werden können “845. „Es ist damit für Provider empfehlenswert, Nutzern (der Betroffene bzw. der Verletzte) die Möglichkeit zu geben, auf einfachem Weg Verletzungen ihrer Schutzrechte auf der Plattform aufzufinden, die der Provider sodann nach entsprechender Monierung löscht. ${ }^{\text {"846 }}$

Aufgrund dieser Entscheidung ist es ein Abwägungselement der Zumutbarkeit geworden, ob der Provider dem Betroffenen die Möglichkeit einer Selbstkontrolle anbietet. ${ }^{847}$ Hätte der Betroffene diese Möglichkeit, wäre die Kontrolle derselben Inhalte für den Provider weniger zumutbar.

Das „Notice-and-Stay-Down“-Verfahren i.S.v. dieser Entscheidung ist nämlich eine Kombination mit einem System, mit dem der Betroffenen auf einfachem Weg die gleichartigen Rechtsverletzungen selbst aufspüren kann, und mehreren "Notice-and-Take-Down“-Verfahren, mit dem der Verletzte vom ISP verlangen kann, ohne Einsetzen des Kommunikationsprozesses die aufgespürten Rechtsverletzungen zu stoppen. Mit dem Anbieten eines Selbstkontroll-Systems hat der ISP die Möglichkeit, die Pflicht zur Entdeckung zukünftiger derartiger Rechtsverletzungen auf den Betroffenen zu übertragen und dadurch die wegen Unterlassungsansprüchen entstehenden Prüfungskosten im großen Umfang einzusparen.

\section{d) Schadensersatz- und Schmerzensgeldansprüche gegen den ISP wegen Pflichtverletzung}

Eine Störerhaftung eröffnet gegen den Provider einen Beseitigungs- oder Unterlassungsanspruch, niemals unmittelbar einen Schadensersatzanspruch. ${ }^{848}$

\footnotetext{
843 Vgl. Spindler, MMR 2007, 507, 514.

844 BGH, K\&R 2011, 117, $120 \mathrm{f}$.

845 Volkmann, K\&R 2012, 381383.

846 Volkmann, K\&R 2012, 381383.

847 Volkmann, K\&R 2012, 381383.

848 Vgl. BGH, GRUR 2004, 860, 864; BGH, GRUR 2002, 618, 619.
} 
Dennoch besteht ab der dem Provider bekannt gewordenen Rechtsverletzung eine Beseitigungspflicht, eine auf die zukünftigen gleichartigen Verletzungen gerichtete Untersuchungspflicht und ggf. eine damit verbundene Beseitigungspflicht des ISP ${ }^{849}$ Bei Nichterfüllung dieser Pflichten kann der Betroffene wegen Pflichtverletzung gemäß $\$ 280$ I BGB Schadensersatz von dem Provider verlangen ${ }^{850}$ wenn es zu einer Rechtsverletzung des Betroffenen kommt, die hätte vermieden bzw. hätte geringer gehalten werden können, wenn der Provider die Pflichten erfüllt hätte. ${ }^{851}$

Zu bemerken ist es, dass ein Schadensersatzanspruch gemäß $\$ 10$ Satz 1 Nr. 1 TMG auch begründet wäre, wenn der Provider die rechtswidrige Handlung oder die Information wegen der Offensichtlichkeit kennen sollte.

Dieser Schadensersatzanspruch gegen den Provider stammt nicht aus seiner Verantwortung für fremde Informationen als Störer, sondern aus der Verletzung seiner Handlungspflichten, die aus der Störerhaftung einen Schritt weiter entwickelt worden sind. ${ }^{852}$

Im Fall der Verletzung dieser Handlungspflicht kann auch ein Schmerzensgeldanspruch gegen den ISP bestehen, wenn dieser Anspruch gegen den unmittelbaren rechtsverletzenden Internetnutzer begründet ist. Der ISP muss für wegen seiner Pflichtverletzung entstehenden oder erweiterten Schaden haften.

\section{Nach chinesischem Recht}

\section{Die Reihenfolge der Ansprüche gegen den unmittelbaren rechtsverletzenden Internetnutzer und den ISP}

Gemäß $₫ 36$ Delikthaftungsgesetz haftet der ISP gesamtschuldnerisch mit dem rechtsverletzenden Internetnutzer. Deswegen gibt es für die Ansprüche gegen die beiden keine Reihenfolge. Ungünstig für den Betroffenen wäre es aber, wenn er zwingend den ISP und den Internetnutzer gleichzeitig anklagen muss.

Wie vorher bereits diskutiert wurde, liegt der Grund der gesamtschuldnerischer Haftung des ISP nach der herrschenden Meinung an seiner Mittäterschaft mit dem unmittelbaren rechtsverletzenden Internetnutzer. Gemäß $₫ 5$ „Erklärung einiger Fragen über die Gesetzesanwendung für die Beurteilung der Fälle

849 Ensthaler/Heinemann, GRUR 2012, 433, 440.

850 Ensthaler/Heinemann, GRUR 2012, 433, 440; Vgl. Westermann in Erman, BGB $\$ 280$, Rn. 6.

851 Ensthaler/Heinemann, GRUR 2012, 433, 439.

852 Ensthaler/Heinemann, GRUR 2012, 433, 439. 
über den aufgrund von personenbezogenen (körperliche und geistliche) Rechtsverletzungen entstehenden Schadenersatzanspruch ${ }^{\text {“853 }}$ soll das Gericht in diesem Fall von Amts wegen die anderen Täter als Mitbeklagte hinzufügen, wenn der Rechtsinhaber nur gegen einen Teil der Täter wegen Rechtsverletzung klagt. Nach $\$ 53$ chinesischen Zivilprozessgesetzes ist diese Klage eine notwendige gemeinsame Klage ${ }^{854}$ Für eine notwendige gemeinsame Klage müssen alle Beklagten an der Gerichtshandlung teilnehmen. Sonst wäre die Gerichtshandlung prozesswidrig.

Im Fall der Menschenfleischsuche müssen dann der ISP und der unmittelbarere rechtsverletzende Internetnutzer bei der Gerichtshandlung da sein. Hier entstünde wegen der Anonymität und der Pseudonymität wiederum die Frage der Identifizierung des beklagten Internetnutzers im Gerichtsprozess, die, wie oben gesagt wurde, immer noch eine ungelöste Frage ist. ${ }^{855}$ Falls der Kläger auf die Ansprüche gegen den schwierig identifizierbaren Internetnutzer verzichtete, verzichtete er gemäß $₫ 5$ „Erklärung einiger Fragen über die Gesetzesanwendung für die Beurteilung der Fälle über den aufgrund von personenbezogenen (körperliche und geistliche) Rechtsverletzungen entstehenden Schadenersatzanspruch ${ }^{\text {"856 }}$ gleichzeitig auf den Teil vom Schadenersatz, für den der Internetnutzer haften sollte. Für diesen Teil haftete der ISP auch nicht mehr.

Die Regelungen würden den Betroffenen beim Schutz seines Rechts vor dem Gericht offensichtlich in Schwierigkeit bringen, wenn sie so wie oben eng ausgelegt würden. Um einen besseren Schutz des Betroffenen zu schaffen, haben die Gerichte in der Praxis einen abweichenden Weg genommen. Wenn der Betroffene gegen den ISP und den unmittelbaren Verletzer als Beklagte klagt, wird der Prozess vom Gericht als eine normale gemeinsame Klage behandelt. Wenn der Betroffene nur gegen den ISP klagt, wird das Gericht den unmittelbaren Verletzer nicht als weiteren Beklagten hinzufügen. Die Ansprüche gegen den unmittelbaren Verletzer werden auch nicht als verzichtet behandelt. ${ }^{857}$

Eine abweichende Auslegung der gesetzlichen Regelungen in der Entscheidungspraxis ist in diesem Fall durchaus möglich, weil das Verhältnis zwischen dem ISP und dem unmittelbaren Verletzer mit dem typischen Verhältnis zwischen den Mittätern nicht völlig identisch ist. Wie bereits erwähnt, wird dem ISP die gesamtschuldnerische Haftung unter Berücksichtigung der öffentlichen

853 Vom chinesischen Obersten Volksgerichtshof.

854 Yao Hong, S. 74.

855 Siehe oben unter $₫ 11$ II 2.

856 Vom chinesischen Obersten Volksgerichtshof.

857 Chen Jinchuan, Journal of Law Application 2011, No. 6, 52, 56. 
Politik auferlegt, weil dadurch das Recht des Verletzten im Internet am besten geschützt werden kann. Die zivilprozessrechtlichen Regelungen in China sind aufgrund der schnellen Entwicklung des Internets nicht mehr aktuell, und müssen geändert werden. ${ }^{858}$

\section{Der Beseitigungsanspruch gegen den ISP}

Der Beseitigungsanspruch gegen den ISP in China findet seine Grundlage in $\$ 36$ Abs. 2 Delikthaftungsgesetz, gemäß dem der Verletzte das Recht hat, den ISP $\mathrm{zu}$ informieren und nötige Maßnahmen vom ISP zu verlangen, um den rechtswidrigen Inhalt zu löschen, zu sperren oder das Verlinken zu dem Inhalt abzubrechen, wenn ein Internetnutzer mit Benutzung des Internets eine Rechtsverletzung begangen hat. Der vom $₫ 36$ Abs. 2 Delikthaftungsgesetz geregelte Beseitigungsanspruch ist eigentlich eine Übernahme des „Notic-and-TakeDown“-Verfahrens von den USA. ${ }^{859}$

\section{a) Über Notice}

Ein qualifiziertes Notice ist eine Voraussetzung des Beseitigungsanspruchs. Um das „Notice-and-Take-Down“-Verfahren erfolgreich durchzuführen, soll der ISP gemäß $\$ 9$ Abs. 1 Nr. 5 „Regelung einiger Fragen über die Gesetzanwendung auf die Beurteilung der Fälle über den zivilrechtlichen Rechtsstreit wegen Verletzung des informationellen Verbreitungsrechts im Internet" ein funktioniertes Programm entwickeln, um damit Notice zu bekommen und auf die Notice schnell zu reagieren. Ob so ein Programm existiert, ist ein Standard zu beurteilen, ob der ISP während „Notice-and-Take-Down“-Verfahren Schuld hat.

Gemäß $₫ 13$ der oben genannten Regelung kann das Notice in Form von Brief, Fax oder E-Mail sein. Nach dem Erlangen des Notice soll der ISP rechtzeitig passende Maßnahmen vornehmen. Um zu beurteilen, ob die von dem ISP vorgenommenen Maßnahmen rechtzeitig sind, soll das Gericht mit Berücksichtigung der Form und der Ausführlichkeit des Notice, der Schwierigkeit der vorzunehmenden Maßnahmen, der Art des von dem ISP angebotenen Dienstes, der Art der vom Internetnutzer begangenen Rechtsverletzung, der Anzahl der rechtsverletzenden Inhalte usw. zusammen entscheiden. ${ }^{860}$

858 Chen Jinchuan, Journal of Law Application 2011, No. 6, 52, 56.

859 Xie Xuekai, Oriental Law 2013, No. 2, 149, $154 \mathrm{f}$.

$860 \$ 14$ „Regelung einiger Fragen über die Gesetzanwendung auf die Beurteilung der Fälle über den zivilrechtlichen Rechtsstreit wegen Verletzung des informationellen Verbreitungsrechts im Internet" vom chinesischen Obersten Volksgerichtshof. 


\section{b) Über „Anti-Notice“}

Das Delikthaftungsgesetz hat jedoch das „Notice-and-Take-Down“-Verfahren von den USA nicht komplett übernommen, weil die Regelung von $\$ 36$ Delikthaftungsgesetz nur zugunsten des Betroffenen ist. Ein „Anti-Notice“-Verfahren zugunsten des Äußernden, der die vermutlich rechtsverletzenden Inhalte veröffentlicht hat, ist vom Delikthaftungsgesetz vernachlässigt.

Ein „Anti-Notice“-Verfahren ist aber in der „Verordnung zum Schutz des informationellen Verbreitungsrechts im Internet“ - eine Verordnung über Urheberrecht - geregelt. Aufgrund $\$ 16$ dieser Verordnung kann der verdächtige Verletzer schriftlich beim ISP beantragen, die gelöschten oder gesperrten Werke oder Links widerherzustellen, wenn er nach dem Erhalt der Beanstandung des Rechtsinhabers daran glaubt, dass die von ihm angebotenen Werke keine Rechtsverletzung begründen. Der Antrag des verdächtigten Verletzers soll nach $\$ 16$ Abs. 2 der Verordnung durch grobes Beweismaterial unterstützt werden. Der ISP soll nach $\$ 17$ der Verordnung das bezügliche Werk sofort wiederherstellen, nachdem er den schriftlichen Antrag bekommen hat. Gleichzeitig soll der ISP den Antrag an den Rechtsinhaber weiterleiten. Eine weitere Beanstandung an dem gleichen Werk von dem gleichen Rechtsinhaber wird nicht mehr berücksichtigt.

Jedoch beschränkt sich diese Verordnung nur auf Urheberrechtsverletzungen, während $\$ 36$ i.V.m. $\$ 2$ Delikthaftungsgesetz für jede Art von Rechtsverletzungen gilt. Deswegen entsteht die Frage, ob es möglich ist, für das im Bereich des Urheberrechts geregelte „Anti-Notice“-Verfahren eine analoge Anwendung auf dem Schutz des Persönlichkeitsrechts zu finden. ${ }^{861}$ Auf diese Frage gibt es in der Literatur hauptsächlich Gegenmeinungen.

Der Grund für die Gegenmeinungen liegt hauptsächlich darin, dass es für die Beurteilung von Persönlichkeitsrechtsverletzungen und Urheberrechtsverletzungen unterschiedliche Kriterien gebe. ${ }^{862}$ Das Beweisen ${ }^{863}$ und das Identifizieren $^{864}$ der Urheberrechtsverletzung seien relativ einfach, weil die Dimension des Urheberrechts relativ klar sei. Im Gegensatz dazu sei die Dimension der Persönlichkeitsrechte relativ undeutlich. Auch die Gerichte müssen unter

861 Cai Chang, Studies in Law and Business 2013, No. 2, 113, 115.

862 Yuan Xueshi, Political Science and Law 2008, No. 4, 19, 22; Lu Chunya, Journal of Henan University of Economics and Law 2012, No. 5, 58, 60; Mei Xiaying/Liu Ming, Science of Law 2013, No. 2, 82, 90.

863 Cai Chang, Studies in Law and Business 2013, No. 2, 113, 116.

864 Mei Xiaying/Liu Ming, Science of Law 2013, No. 2, 82, 90. 
Berücksichtigung der Konstellation in jedem einzelnen Fall eine Interessenabwägung durchführen, um eine Entscheidung der Rechtsverletzung zu treffen. ${ }^{865}$ Dafür sei der ISP nicht unbedingt in der Lage. ${ }^{866}$

Diese Gegenmeinung ist nicht überzeugend, weil der ISP beim Notice im Fall der Persönlichkeitsrechtsverletzung bereits überprüfen muss, ob der Inhalt überzeugend ist. Wäre er für „Anti-Notice“ nicht in der Lage, sollte der Gesetzgeber ihm auch diese Überprüfungspflicht bei Notice nicht geben. Übrigens kommt es für die Beurteilung einer Verletzung auf die Ausführlichkeit des Notice an. Auch für die Persönlichkeitsrechtsverletzung könnte es einen einfach zu beurteilenden Fall geben. Übrigens wird vom ISP bei der Beurteilung auch nur eine allgemeine Sorgfaltspflicht erwartet, der Betroffene und der Äußernde sollen mehr für die Wahrheit ihrer Stellungnahmen verantwortlich sein. ${ }^{867}$

Es herrscht jedoch die Meinung, dass das Dilemma der Analogisierung völlig vernachlässig werden kann. Nach dieser Meinung ist eine oben genannte Analogisierung gar unnötig, weil ein „Anti-Notice“-Verfahren für jede Rechtsverletzung im Sinne von $\$ 36$ i.V.m. $\$ 2$ Delikthaftungsgesetz ein impliziertes "Muss" ist. ${ }^{868}$ Ohne das „Anti-Notice“-Verfahren würde das Recht des Rechtinhabers sehr leicht missbraucht, weil die der Rechtsverletzung verdächtigten Inhalte nur auf Verlangen des Rechtsinhabers ohne inhaltliche Prüfung gesperrt oder gelöscht würden. ${ }^{869}$ Die Stimme der Gegenseite würde in diesem Fall gar nicht angehört werden. Ihre Rechte und Freiheiten würden völlig vernachlässigt, zu denen insbesondere die Meinungsfreiheit gehört. Ohne "Anti-Notice“-Verfahren bestände die Gefahr der Verfassungswidrigkeit des Delikthaftungsgesetzes, weil die Meinungsfreiheit durch das Delikthaftungsgesetz unter alle anderen Rechte gesetzt würde, was offensichtlich gegen $\$ 51$ chinesisches Verfassungsgesetzes widrig wäre, aufgrund dessen das Ausüben der Rechte und Freiheiten von einem Bürger die Rechte und Freiheiten der anderen nicht verletzen darf.

Es ist in der Literatur allgemein anerkannt, dass das Fehlen des „Anti-Notice“Verfahrens im Delikthaftungsgesetz ein Fehler des Gesetzgebers ist. Diese Lücke soll entweder durch die Rechtsprechung oder durch die Novellierung des Gesetzes erfüllt werden.

865 Zhou Hua, Journal of China Three Gorges University (Humanities \& Social Sciences) 2012, No. 5, 87, 89.

866 Mei Xiaying/Liu Ming, Science of Law 2013, No. 2, 82, 84, 90.

867 Xie Xuekai, Oriental Law 2013, No. 2, 149, 154 f.

868 Yang Lixin/Li Jialun, Science of Law 2012, No. 2, 157, 157.

869 Yang Lixin/Li Jialun, Science of Law 2012, No. 2, 157, 158. 


\section{c) Die Kommentare über das chinesische}

„Notice-and-Take-Down"-Verfahren

Gleich wie die Kritiken gegen den Kommunikationsprozess gibt es in der chinesischen Literatur auch zahlreiche Kritiken gegen das „Notice-and-Take-Down“Verfahren. Viele Kritiken sind mit denselben Gründen wie in Deutschland argumentiert. Im Folgenden werden nur die Argumente mit chinesischen Besonderheiten diskutiert.

\section{aa) Die Gefahr einer haftungsrechtlichen Zwickmühle für den ISP}

Es ist nicht zu verneinen, dass es manchmal Fälle von Rechtsverletzungen gibt, die schwierig zu beurteilen sind. Wenn der ISP zwingend eine Entscheidung treffen muss, könnte er entweder die rechtsverletzenden Fälle als nicht rechtsverletzend fehlerhaft entscheiden und wegen Pflichtverletzung gesamtschuldnerisch haften, ${ }^{870}$ oder die nicht rechtsverletzenden Fälle als rechtsverletzend fehlerhaft entscheiden und wegen vertraglicher Pflichtverletzung haften. Für die schwierig zu entscheidenden Fälle könnte der ISP in eine haftungsrechtliche Zwickmühle geraten.

Die Zwickmühle ist sogar in der Entscheidungspraxis in China tatsächlich passiert. Im Jahr 2011 hat Frau Fang Jing beim Betreiber der MikrobloggingWebseite "Sina Weibo“ beanstandet, dass Frau Yu auf ihrem Blog rechtsverletzende Inhalte über sie geschrieben hat. „Sina Weibo" hat deswegen den Account von Frau Yu gesperrt. Nach der Beurteilung des Gerichts hat die Äußerung von Frau Yu auf ihrem Blog die Grenze der Meinungsfreiheit nicht überschritten. „Sina Weibo“ musste wegen vertraglicher Pflichtverletzung die wegen des Gerichtsprozesses entstehenden Kosten für Frau Yu übernehmen. „Sina Weibo“ hat während des Prozesses behauptet, dass sein Dienst kostenlos ist, und er deswegen ohne Grund den Dienst jeder Zeit beenden kann. Nach der Entscheidung kann „Sina Weibo“ nicht damit argumentieren, dass sein Dienst kostenlosen ist, weil er wegen Werbung auf der Webseite, orientierte Werbung per E-Mail zu den Nutzern usw. tatsächlich von den Nutzern profitiert. Ein Dienstvertrag kommt zwischen "Sina Weibo" und den Nutzern zustande.

Angeblich hat diese Entscheidung den ISP zu einer relativ strengen Pflicht verurteilt. Aber der Grund dieser Entscheidung liegt meiner Meinung nach eher darin, dass ein „Anti-Notice“-Verfahren für die Persönlichkeitsrechtsverletzung im chinesischen Gesetz fehlt. „Sina Weibo“ hat nach der Beanstandung von Frau

870 Zhou Hua, Journal of China Three Gorges University (Humanities \& Social Sciences) 2012, No. 5, 87, 90. 
Fang Jing schnell reagiert, hat aber das Verlangen von Frau Yu auf Wiederherstellung ihres Accounts vernachlässigt, weil eine Pflicht zur Wiederherstellung nicht im Gesetz vorgeschrieben ist.

Das Fehlen des „Anti-Notice“-Verfahrens bzw. der aus diesem Verfahren entstehenden Pflicht des ISP könnte leicht dazu führen, dass der ISP die Beanstandung des Betroffenen ohne Prüfung zu 100\% akzeptieren könnte, um eine gesamtschuldnerische Haftung zu vermeiden. ${ }^{871}$ Das wäre offensichtlich eine starke Beschränkung der Meinungsfreiheit. ${ }^{872}$

\section{bb) Die Unfähigkeit des ISP bei der Beurteilung komplexer Persönlichkeitsrechtsverletzung}

Nicht nur in China sondern auch in Deutschland ist es eine entscheidende Frage, was der ISP zum Schluss des „Notice-and-Take-Down“-Verfahrens oder des Kommunikationsprozesses machen soll, wenn die Konstellation so kompliziert ist, dass der ISP die Persönlichkeitsrechtsverletzung nicht beurteilen kann.

Bei dieser Diskussion im deutschen Teil habe ich vorgeschlagen, dass der ISP in diesem Fall nicht zwingend eine Entscheidung zugunsten einer Partei treffen muss, sondern den Parteien mitteilen soll, sich direkt an das Gericht zu wenden. Auch nachdem der ISP eine Entscheidung gegeben hat, soll er nicht für seinen Fehler haften, solange er die allgemeine Sorgfaltspflicht bei der Überprüfung nicht verletzt hat. ${ }^{873} \mathrm{Ob}$ es sich um einen schwierigen Fall handelt, soll nach der Konstellation betrachtet werden, ob ein Rechtsverstoß so offensichtlich ist, dass auch eine durchschnittliche verständige Person die Rechtsverletzung unter Berücksichtigung der Situation unschwer bejahen kann. Die Offenlegung der Intimsphäre einer normalen Person z.B. soll zur offensichtlichen Rechtsverletzung gehören. Dieser Vorschlag wird nicht dazu führen, dass der ISP jede Entscheidungsfindung ablehnt, denn ist eine Rechtsverletzung nach der Entscheidung des Gerichts offensichtlich, muss der ISP wegen Pflichtverletzung haften.

871 Civil Law Department of Legislative Affairs Commission of NPC Standing Committee, S. 588, 611, 620f.; vgl. Zhou Hua, Journal of China Three Gorges University (Humanities \& Social Sciences) 2012, No. 5, 87, 89; Mei Xiaying/Liu Ming, Science of Law 2013, No. 2, 82, 84.

872 Vgl. Xie Hongfei, Procuratorial View 2010, No. 3, 26; Zhou, Bo/Yang, Kangrui, Chinese Journal of Law 2012, No. 1, 108, 114; Cai Chang, Studies in Law and Business 2013, No. 2, 113, 114f.; Yang Lixin/Li Jialun, Science of Law 2012, No. 2, 157, 158.

873 Yang Lixin/Li Jialun, Science of Law 2012, No. 2, 157, 162; Mei Xiaying/Liu Ming, Science of Law 2013, No. 2, 82, 84. 


\section{cc) Die Möglichkeit, von der Quelle an die Rechtsverletzungen zu vermindern}

Der ISP ist prinzipiell Mittäter mit dem unmittelbaren rechtsverletzenden Internetnutzer. Jedoch ist der ISP als Verletzer im Vergleich mit dem rechtsverletzenden Internetnutzer einfacher zu finden. Gleichzeitig hat er auch die größere Fähigkeit, die Schäden des Verletzten zu ersetzen. Der vernünftige Verletzte würde sich lieber direkt an den ISP wenden, um Schadensersatz zu verlangen, als den Internetnutzer als unmittelbaren Verletzer zu suchen. Obwohl es gesetzlich geregelt ist, dass der ISP von dem unmittelbaren Verletzer den von ihm haftenden Teil verlangen kann, ist es in der Praxis aber schwierig zu verwirklichen. ${ }^{874}$ Das alles gibt dem Internetnutzer die Chance, eine Haftung wegen seiner rechtswidrigen Handlung zu vermeiden. Seine Kosten für rechtswidrige Handlungen sind im großen Maße vermindert. ${ }^{875}$

Das „Notice-and-Take-Down“-Verfahren hat tatsächlich die Kosten des Internetnutzers wegen seiner rechtswidrigen Handlung durch die gewerblichen Kosten des ISP zur Kontrolle rechtswidriger Inhalte auf seiner Webseite ersetzt. ${ }^{876}$ Nach der Theorie der ökonomischen Analyse des Rechts soll derjenige haften, der mit geringsten Kosten das rechtsverletzende Ergebnis vermeiden kann. ${ }^{877}$ Für die Internetnutzer liegen die Transaktionskosten auf einem direkten Zivilprozess gegen ihn, um eine von ihm begangene Rechtsverletzung zu beurteilen. Im Gegensatz liegen die Transaktionskosten beim ISP im Fall von „Notice-andTake-Down“-Verfahren auf der Bereitstellung Fachleute, um „Notice“ über Rechtsverletzungen zu bearbeiten, die möglichen Rechtsverletzungen zu beurteilen, und gegebenenfalls den rechtswidrigen Inhalt zu löschen. Dazu kommt noch der mögliche Zivilprozess gegen den ISP wegen Pflichtverletzung und gegebenenfalls eine Klage gegen den Internetnutzer als der tatsächliche Verletzer, um den vom ISP zuerst bezahlten Schadensersatz zu übernehmen. Offensichtlich werden durch das „Notice-and-Take-Down“-Verfahren zu viele Umwege gebaut. Ökonomisch betrachtet soll in diesem Fall der Internetnutzer direkt der Träger der Haftung sein. ${ }^{878}$

874 Zhou Hua, Journal of China Three Gorges University (Humanities \& Social Sciences) 2012, No. 5, 87, 89.

875 Mei Xiaying/Liu Ming, Science of Law 2013, No. 2, 82, 89.

876 Mei Xiaying/Liu Ming, Science of Law 2013, No. 2, 82, 85

877 Mei Xiaying/Liu Ming, Science of Law 2013, No. 2, 82, 85

878 Mei Xiaying/Liu Ming, Science of Law 2013, No. 2, 82, 85 
$\$ 36$ des Delikthaftungsgesetzes hat durch das „Notice-and-Take-Down“Verfahren die Last dem ISP gegeben. Als Ergebnis würde der Internetnutzer eine rechtswidrige Handlung wegen ihrer geringen Kosten leicht begehen, während der ISP eine schwere Pflicht zur Kontrolle der rechtswidrigen Inhalte und ein hohes Risiko für die falsche Beurteilung tragen muss.

Das „Notice-and-Take-Down“-Verfahren ist wegen der Besonderheit im Internet für den schnellen Schutz des Persönlichkeitsrechts des Betroffenen vor vertiefter Rechtsverletzung effizient, aber nicht geeignet, die Rechtsverletzungen von der Quelle an zu vermeiden oder eine gesunde Kultur im Internet aufzubauen. ${ }^{879}$

\section{Der Unterlassungsanspruch gegen den ISP}

Ein direkter Ausdruck über einen Unterlassungsanspruch besteht in $₫ 9$ Abs. 1 Nr. 6 „Regelung einiger Fragen über die Gesetzesanwendung auf die Beurteilung der Fälle über den zivilrechtlichen Rechtsstreit wegen Verletzung des informationellen Verbreitungsrechts im Internet", wonach der Verletzte nach der ersten Rechtsverletzung das Recht hat, vom ISP zu verlangen, passende Maßnahmen vorzunehmen, um die wiederholte Rechtsverletzung durch den gleichen Nutzer zu vermeiden oder rechtzeitig zu stoppen. Sonst wird die Kenntnisnahme des ISP über die Rechtverletzung gesetzlich vermutet. Die Schuld des ISP wird deswegen begründet; er muss dann gemäß $₫ 8$ Abs. 1 Satz 2 dieser Regelung als Gehilfe haften.

Aber als Grundlage des Unterlassungsanspruchs ist diese als Rechtsprechung erlassene Regelung nicht in der Lage. Meiner Meinung nach korrespondiert $\$ 9$ Abs. 1 Nr. 6 i.V.m. $\$ 8$ Abs. 1 Satz 2 der oben genannten Regelung mit $₫ 36$ Abs. 3 Delikthaftungsgesetz, das als Grundlage des Unterlassungsanspruchs gegen den ISP angesehen werden kann. Gemäß $₫ 36$ Abs. 3 Delikthaftungsgesetz soll der ISP mit demjenigen Internetnutzer gesamtschuldnerisch für den gesamten Schaden haften, wenn der ISP wusste, dass der Internetnutzer unter Benutzung seines Internetdienstes die zivilrechtlichen Rechte oder Interessen des anderen verletzt und keine nötigen Maßnahmen vorgenommen hat. Diese Paragraphen können kombiniert verwendet werden. Nach der ersten Rechtsverletzung soll die Kenntnisnahme des ISP über die wiederholte Rechtsverletzung durch den gleichen Nutzer vermutet werden. Der ISP soll nötige Maßnahmen vornehmen; sonst muss er als Gehilfe gesamtschuldnerisch haften, weil hier ein bewusstes Nicht-Verhalten besteht, damit eine Garantenstellung begründet werden kann.

879 Mei Xiaying/Liu Ming, Science of Law 2013, No. 2, 82, 85 
Ein Unterlassungsanspruch im Fall von Persönlichkeitsrechtsverletzung ist in China relativ neu. Die „Regelung einiger Fragen über die Gesetzesanwendung auf die Beurteilung der Fälle über den zivilrechtlichen Rechtsstreit wegen Verletzung des informationellen Verbreitungsrechts im Internet", die direkt den Unterlassungsanspruch regelt, ist auch erst seit 1.1.2013 in Kraft getreten. Über dieses Thema fehlen in der Literatur und in der Praxis Diskussionen.

Aber eine Anlehnung an das deutsche Recht könnte in diesem Fall durchaus möglich sein, weil das am 1.10.2007 in Kraft getretene chinesische Sachenrechtgesetz die Struktur des Sachenrechts im deutschen BGB übergenommen hat, und $₫ 35$ des chinesischen Sachenrechtgesetzes eine direkte Übersetzung von $\$ 1004$ Abs. 1 BGB ist, wo die Grundlage des Unterlassungsanspruchs liegt.

\section{Der Schadensersatzanspruch und der Schmerzensgeldanspruch gegen den ISP}

Hätte der ISP die Beseitigungs- oder Unterlassungspflicht vorsätzlich oder fahrlässig verletzt, könnte der Betroffene Schadensersatz oder Schmerzensgeld von ihm verlangen. ${ }^{880}$ Wegen der gesamtschuldnerischen Haftung gelten für die weiteren Voraussetzungen eines Schadensersatz- oder Schmerzensgeldanspruchs gegen den ISP die gleich Prinzipien wie gegen den unmittelbaren rechtsverletzenden Internetnutzer. ${ }^{881}$

Obwohl Deutschland und China bei der Beurteilung der Schadensersatz- und Schmerzensgeldansprüche ähnliche Regelungen haben, führt dies wegen der unterschiedliche Kriterien bei der Beurteilung der Verletzung der Beseitigungsund Unterlassungspflicht zu unterschiedlichen Ergebnisse. In Deutschland beschränkt sich die Beseitigungs- und Unterlassungspflicht auf positive Kenntnis der Rechtsverletzung. Im Gegensatz dazu besteht die Handlungspflicht des ISP in China auch auf der Rechtsverletzung, die er aufgrund des Umstandes kennen soll.

Die gesetzliche Gestaltung in China könnte dazu führen, dass der ISP ein hohes Risiko für die von seinen Nutzern begangene Rechtsverletzung tragen muss. ${ }^{82}$ Als unmittelbares Ergebnis dieser Wirkungen könnte der ISP in China die allgemeine Überwachungspflicht tatsächlich tragen müssen. Dies wäre offensichtlich mit der Tendenz der Entwicklung des Internets und auch der aktuellsten Rechtsprechung des Obersten Volksgerichtshofes nicht zu vereinbaren. ${ }^{883}$

880 Vgl. Liu Xiaochun, Internet Law Review 2011, No. 1, 3, 8, 15.

881 Siehe oben unter $\$ 11$ I 2 a) und b).

882 Mei Xiaying/Liu Ming, Science of Law 2013, No. 2, 82, 87.

883 Siehe oben unter $\$ 10$ II 1. 


\section{Eine Rechtsvergleichende Zusammenfassung}

Die Providerhaftung bietet dem Verletzten Schutz aus drei Aspekten, nämlich die laufende Rechtsverletzung rechtzeitig zu stoppen, den entstehenden Schaden $\mathrm{zu}$ ersetzen und die zukünftige gleichartige Rechtsverletzung zu vermeiden.

\section{1. Über Beseitigungsanspruch}

Um dielaufende Rechtsverletzung rechtzeitig zu stoppen ist durch den Beseitigungsanspruch verwirklicht. Dafür hat Deutschland „Notice-and-Action“-Verfahren bzw. Kommunikationsprozess und China „Notice-and-Take-Down“-Verfahren zur Verfügung gestellt. Die beiden Verfahren stammen aus dem „Notice-andTake-Down“-Verfahren in den USA, das den ISP auf dem Kernpunkt des Anspruchsprozesses stellt. Funktional betrachtet dienen die beiden Verfahren dazu, die Rechte des Verletzten ohne Gerichtsverfahren effizient zu schützen.

Im Vergleich mit dem „Notice-and-Action“-Verfahren in Deutschland ist das Chinesische "Notice-and-Take-Down“-Verfahren zum Schutz des Verletzten effizienter, weil ein „Anti-Notice“-Verfahren vernachlässigt wird. Dies könnte aber zur Beschränkung der Meinungsfreiheit des Äußernden führen. Als Ausgleich kann der Äußernde jedenfalls Delikthaftung wegen falsches „Notice“ gegen den „Notice-Geber (Melder)“ verlangen.

Der Kommunikationsprozess erfordert nicht, dass der ISP nach der Beanstandung des Betroffenen den bezüglichen Inhalt sofort sperrt. Das bietet zwar einen guten Schutz der Meinungsfreiheit, aber im Fall der schwerwiegenden Persönlichkeitsrechtsverletzung wird der Betroffene nicht rechtzeitig genug geschützt. Der Kommunikationsprozess soll für diesen Sonderfall eine vorläufige Beschränkung der Meinungsfreiheit bejahen.

Von den beiden Verfahren sind Verbesserungen zu erwarten. Idealerweise wäre eine Kombination beider Verfahren, dass für den Sonderfall wie zum Beispiel im Fall der schwerwiegenden Persönlichkeitsrechtsverletzung ein einstweiliges schnelles „Take-Down“ Vorrang hat, während für normale Situationen ein „Anti-Notice“ berücksichtigt werden muss.

\section{2. Über Schadensersatz- und Schmerzensgeldansprüche}

Die Beurteilung der Schadensersatz- und Schmerzensgeldansprüche gegen den ISP als Störer setzt in Deutschland und China voraus, dass der ISP die Beseitigungs- oder Unterlassungspflicht verletzt hat. In den beiden Ländern ist es anerkannt, dass eine Handlungspflicht des ISP besteht, wenn er die Rechtsverletzung den Umständen nach kennen soll. Von dieser Hinsicht gibt es in den beiden Ländern kaum ein Unterschied. 
Aber für die Beurteilung, wann der ISP eine Rechtsverletzung kennen soll, hat China strengere Regeln entwickelt. Dies könnte dazu führen, dass der ISP in China tatsächlich die allgemeine Überwachungspflicht trägt.

\section{3. Über Unterlassungsanspruch}

Um die zukünftige gleichartige Rechtsverletzung zu vermeiden wurden in Deutschland ausführliche Theorien über Unterlassungsanspruch entwickelt.

Anders als in Deutschland gibt es in China kein Konzept wie Unterlassungsanspruch gegen den Provider. Ein Unterlassungsanspruch gegen den Provider wird in China im Rahmen der Delikthaftung bzw. der Mittäterhaftung des Providers betrachtet. Wenn eine vom Internetnutzer begangene Rechtsverletzung eine wiederholte Rechtsverletzung ist, soll der Provider über die Rechtsverletzung wissen und sich ohne „Notice“ des Verletzten aktiv verhalten, die Inhalte zu sperren oder entfernen, um eine gesamtschuldnerische Haftung mit dem unmittelbaren Verletzer zu vermeiden.

Durch diesen Weg kann in China das gleiche Ergebnis im Sinne vom Schutz der Verletzten erreicht werden. Aber weil die allgemein anerkannten Grundtheorien über Unterlassungsanspruch fehlen, ist es nicht zu vermeiden, willkürliche Entscheidungen bei der Beurteilung der Pflichte des Providers zu treffen.

Jedoch ist eine Anlehnung des chinesischen Rechts am deutschen Recht in diesem Fall möglich, weil das chinesische Sachenrechtgesetz die Struktur vom Sachenrecht im deutschen BGB übergenommen hat und $\$ 35$ chinesisches Sachenrechtgesetzes eine direkte Übersetzung von $\$ 1004$ Abs. 1 BGB ist, wo die Grundlage des Unterlassungsanspruchs geregelt ist.

Bezüglich des Unterlassungsanspruchs ist übrigens der Gedanke des BGH besonders zu empfehlen, dass der ISP ein Selbstkontroll-System dem Betroffenen zur Verfügung stellt, um dem Betroffenen die Aufgabe zu übergeben, die zukünftige gleichartige Rechtsverletzung aufzuspüren. 



\section{$\$ 13$ Die anderen Maßnahmen zum Schutz des Rechtsverletzten während der Menschenfleischsuche}

\section{Ansprüche gegen den ICP und ISP als Täter oder Mittäter}

Im Fall von unmittelbaren Rechtsverletzungen durch den ICP und ISP haften sie als Täter oder Mittäter. ${ }^{884}$ Dementsprechend hat der Verletzte die Ansprüche gegen sie vergleichbar mit den gegen den unmittelbaren rechtsverletzenden Internetnutzer ${ }^{885}$ Wegen mangelnden Besonderheiten werden diese Ansprüche nicht mehr konkret diskutiert.

\section{Selbstregulierung durch Internetnutzer}

Die meisten Menschenfleischsuchen sind von den Internetnutzern unmittelbar eingeleitet und durchgeführt. Deswegen kann es eine wirksame Methode sein, durch die Selbstregulierung das Verhalten der Internetnutzer zu disziplinieren, um die Rechtsverletzungen von der Quelle an zu vermindern.

Am 1.1.2009 hat in China eine Internetgruppe, die "Nichtorganisatorische Alliance der Menschenfleischsuche“ heißt, eine "MenschenfleischsucheKonvention (Version 1.0 Beta)“ in einem bekannten Internetforum „Douban“ veröffentlicht, um an die Internetnutzer zu appellieren, ihr Verhalten während der Menschenfleischsuche selbst zu kontrollieren. ${ }^{886}$

Die Konvention gemäß ihrem Wortlaut zielt darauf, die moralischen Gedanken der Internetnutzer zu wecken, richtiges Verständnis über Menschenfleischsuche zu verbreiten, und die richtige Orientierung der Entwicklung der Menschenfleischsuche zu garantieren.

Die wichtigsten Inhalte bestehen in den folgenden Paragraphen der Konvention:

$\$ 2$. Es wird vorgeschlagen, die Menschenfleischsuche im originalen Sinnen zu benutzen, um Kenntnis zu erlangen.

$\$ 3$. Die Informationen im Bereich der Privatsphäre der Anderen sollen möglicherweise nicht durch Menschenfleischsuche gesucht werden.

884 Siehe oben unter $₫ 9, \S 10$ I 1 und II 3.

885 Vgl. oben unter $\$ 11 \mathrm{I}$.

886 http://www.douban.com/group/topic/5032183/ (besucht am 04.04.2015). 
$\$ 4$. Die Offenlegung der Privatsphäre der Anderen soll möglicherweise verhindert werden. Die Privatsphäre der Anderen soll nicht im öffentlichen Bereich einer Webseite offengelegt werden.

$\S 5$. Für den Fall der Korruption und den Fall von „Strafe den Bösen, Belohne den Guten“ gelten $\$ \$ 3,4$ nicht.

$\$ 6$. Während der Menschenfleischsuche sollen nur die richtigen und glaubwürdigen Informationen veröffentlicht werden. Der Anbieter der Informationen soll für ihre Wahrheit verantwortlich sein.

$\$ 7$. Nicht an der böswilligen Menschenfleischsuche teilnehmen, und Möglicherweise den Betroffenen oder den ISP über die böswillige Menschenfleischsuche informieren. ${ }^{887}$

Diese Konvention hat gezeigt, dass einige Internetnutzer in China die negative Wirkung der Menschenfleischsuche bemerkt haben, und versuchen, die teilnehmenden Internetnutzer der Menschenfleischsuche zu disziplinieren. Jedoch sind nicht alle Inhalte der Konvention mit dem Gesetz zu vereinbaren.

Vom Gesetz zu begrüßen sind die Vorschläge in $\$ 3$ und $\$ 4$, auf die Privatsphäre der Anderen aufzupassen und die Privatsphäre betroffene Information nicht im öffentlichen Bereich offenzulegen. Durch den Vorschlag in $\$ 6$ wird vermieden, aufgrund von falschen Tatsachenbehauptungen eine Ehrverletzung zu begründen. Der Vorschlag von $₫ 7$ garantiert weiterhin den rechtsmäßigen Zweck einer Menschenfleischsuche. Problematisch ist die Regelung in $\S 5$, die die Teilnehmer der Menschenfleischsuche berechtigt, im Fall der Entdeckung von Korruptionen oder im Fall von „Strafe den Bösen, Belohne den Guten“ die Privatsphäre betroffene Information der Zielperson offenzulegen. $\$ 5$ der Konvention hat wiederum das Lynchjustiz-Merkmal der Menschenfleischsuche dargestellt. Die Fähigkeit der Internetnutzer, um „Böse“ und „Gut“ zu beurteilen, wird in $₫ 5$ der Konvention leider nicht ausreichend berücksichtigt.

Immerhin ist die Methode von Selbstregulierung im Vergleich mit den Maßnahmen durch die Regierung milder und von den Internetnutzern leichter $\mathrm{zu}$ akzeptieren. Es ist zu überlegen, durch die Selbstregulierung der Internetnutzer flankiert die gesunde Entwicklung der Menschenfleischsuche anzuleiten. Vorausgesetzt ist jedoch eine besser durchdachte Konvention.

\section{Regulierung durch die ISP}

Mit der Popularisierung der Menschenfleischsuche sind viele ISP motiviert, spezifische Bereiche auf ihren Webseiten für Menschenfleischsuche zur Verfügung

887 http://www.douban.com/group/topic/5032183/ (besucht am 04.04.2015). 
zu stellen. Diese ISP sollen mehr Sorgfaltspflicht über den Inhalt der Menschenfleischsuche als die anderen ISP tragen, auf deren Webseiten die Menschenfleischsuche selten passiert. Für die ISP, die mit Absicht ihre Webseiten für Menschenfleischsuche bereitgestellt haben, ist es vorstellbar, dass sie die allgemeine Überwachungspflicht auf die Inhalte der Menschenfleischsuche haben.

In zwei Entscheidungen über Urheberrecht in China ${ }^{888}$ haben die Gerichte aufgrund der hohen Gefahr der Urheberrechtsverletzung auf der Movie-SharingWebseite festgestellt, dass der ISP von solchen Webseiten eine allgemeine Überwachungspflicht bezüglich der Inhalte hat. ${ }^{889}$ Die Urheberrechtsverletzungen auf der Movie-Sharing-Webseite haben normalerweise wirtschaftliches Interesse als Schutzobjekt, das durch Schadensersatz wiedergutgemacht werden kann, während der Menschenfleischsuche bezüglich hauptsächlich um Persönlichkeitsrechtsverletzungen geht, die starke Prangerwirkung dem Betroffenen bringen könnte, die durch Schadensersatz schlecht wiedergutgemacht werden kann. Übrigens hat Menschenfleischsuche eine hohe Gefahr von Persönlichkeitsrechtsverletzung. ${ }^{890}$ In dieser Hinsicht braucht es für die MenschenfleischsucheWebseite höhere oder mindestens ebenso hohe Überwachungspflichten wie für die Movie-Sharing-Webseite.

Diese Meinung wird in der Praxis tatsächlich von einigen ISP in China akzeptiert, die Menschenfleischsuche-Webseiten betreiben. Der Betreiber von „Mop“, die Webseite aus der die Menschenfleischsuche in China stammt, hat auf der Webseite deutlich geschrieben, dass alle Inhalte, die zur Privatsphäre gehören, während der Menschenfleischsuche gelöscht oder gesperrt werden. ${ }^{891}$ Der Betreiber kontrolliert tatsächlich alle Inhalte während der Menschenfleischsuche. ${ }^{892}$ Der Betreiber von „Douban“, der auch eine Menschenfleischsuche-Webseite zur Verfügung gestellt hat, hat auch Regelungen für Menschenfleischsuche auf seiner Webseite deutlich festgelegt: die Suche nach persönlicher Privatsphäre, einschließlich Name, Kontaktinformation, ID, privates Verhalten, sind verboten;

888 Haidian Unteres Volksgericht, Urt. v. 09.12.2008 - (2008) hai min chu zi di 14025 hao; Shanghai Erstes Mittleres Volksgericht, Urt. v. 23.06.2009 - (2009) hu yi zhong min wu (zhi) zhong zi di 20 hao.

889 Chen Jinchuan, Intellectual Property 2011, No. 2, 56, 60.

890 Vgl. Liu Wenjie, Peking University Law Journal 2012, No. 2, 395, 410.

891 Meng chi xue gao, http://dzh.mop.com/whbm/20080828/0/F53OSOI1b228c4z8. shtml (besucht am 04.04.2015).

892 Meng chi xue gao, http://dzh.mop.com/whbm/20080828/0/F53OSOI1b228c4z8.sht$\mathrm{ml}$ (besucht am 04.04.2015); vgl. Wang Chengwei, Journal of Public Management 2011, No. 4, 21, 29. 
wegen der Schwierigkeit, die Rechtmäßigkeit der Menschenfleischsuche zu beurteilen, darf die Suche nur Prominente als Zielpersonen haben. ${ }^{893}$

Es wird in der Literatur sogar vorgeschlagen, dass der Veranlasser vor der Einleitung einer Menschenfleischsuche erst einen Antrag an den ISP stellen muss, um die Notwendigkeit der Menschenfleischsuche zu beweisen und eine Erlaubnis für die Suche zu erhalten. ${ }^{894}$

Durch die Diskussion in dieser Arbeit soll erkennbar werden, dass die ISP in China eine hohe Pflicht und ein hohes Risiko für die auf ihren Webseiten passierten Rechtsverletzungen tragen müssen. Um die Haftung wegen einer Persönlichkeitsrechtsverletzung durch ihren Nutzer zu erleichtern, ist es für die ISP sinnvoller, Maßnahmen vorzunehmen, die Inhalte der Menschenfleischsuche zu kontrollieren.

Übrigens könnte eine qualifizierte Menschenfleischsuche zahlreiche Teilnehmer bekommen, von denen der ISP gut profitieren kann. Es wird deswegen auch nicht gefordert, dass er eine höhere Überwachungspflicht als ein anderer ISP tragen muss.

\section{Einleitung der Menschenfleischsuche von Journalisten oder zuständigen Behörden}

Wie am Anfang der Arbeit schon erwähnt wurde, gibt es in Deutschland nicht selten Menschenfleischsuche ähnliche Ereignisse. ${ }^{895}$ Der Unterschied zwischen diesen Ereignissen und Menschenfleischsuche liegt darin, dass sie häufig von den Journalisten oder zuständigen Behörden eingeleitet worden sind, und die Offenlegung der Informationen der Zielperson häufig offline durchgeführt wird. Durch diesen Weg können die rechtswidrigen Handlungen während der Suche im großen Maße vermieden werden.

Die Menschenfleischsuchen mit den Motivationen, um Korruptionen oder andere rechtswidrige Verhalten aufzudecken, zielen häufig darauf, die Zielperson zu bestrafen. Dieses Ziel kann ebenfalls erreicht werden, wenn die Internetnutzer die bezügliche Information der Zielperson den zuständigen Behörden offline mitteilen. Das wäre ein idealer Weg, um die Nachteile der Menschenfleischsuche zu vermeiden. Dies setzt jedoch voraus, dass genug Vertrauen vom Volk an die Regierung vorhanden ist, ${ }^{896}$ und die zuständigen Behörden die vorkommenden

893 http://www.douban.com/group/72544/?type=essence (besucht am 04.04.2015).

894 Tian Feilong, Internet Law Review 2009, 80, 91.

895 Siehe oben unter $\$ 1$ VII 2 c).

896 Siehe oben unter $₫ 1$ V 3 b). 
Fälle effizient lösen können. Die Erfüllung dieser Voraussetzungen ist in China zu erwarten.

Die Menschenfleischsuchen mit den Motivationen, um gegen unmoralisches Verhalten zu kämpfen oder um die Internetnutzer zu amüsieren, können idealerweise durch Berichterstattung von den Journalisten eingeleitet werden. Wegen ihrer professionellen Ethik werden die Persönlichkeitsrechte der Zielperson besser berücksichtigt.

Diese Wege sind jedoch nur zu empfehlen und können als Vorbilder der zukünftigen Menschenfleischsuchen berücksichtigt werden. Die durch Internetnutzer eingeleiteten rechtmäßigen Menschenfleischsuchen dürfen nicht zwingend durch diese Wege ersetzt werden. 

Ergebnis 



\section{$\$ 14$ Zusammenfassung und Schlussfolgerungen}

\section{Zusammenfassung}

Menschenfleischsuche ist ein Phänomen weltweit. Wegen der Kombination von komplexen technischen, kulturellen, sozialen, politischen und anderen Hintergründen ist Menschenfleischsuche in China besonders populär. Im Gegensatz dazu ist sie in Deutschland wegen des seit langem entwickelten Gedankens vom persönlichen Datenschutz eher selten praktiziert. Genau deswegen können im entwickelten deutschen Rechtssystem Erfahrungen zur Regulierung der Menschenfleischsuche gefunden werden, weil ein Datenschutzgesetz in China noch fehlt.

Eine Menschenfleischsuche ist nicht unbedingt rechtswidrig. Es kommt auf die Motivationen an. Offensichtlich rechtswidrig ist die Menschenfleischsuche mit der rechtswidrigen Motivation, wie z.B. um Rache auszuüben. Die Rechtswidrigkeit der Menschenfleischsuche soll mit Berücksichtigung der Motivation in jedem einzelnen Fall geprüft werden. Es besteht in China und in Deutschland die Gefahr der Beschränkung der Meinungsfreiheit, wenn man die Menschenfleischsuche allgemein verbietet.

Jedoch besteht für Menschenfleischsuche eine hohe Gefahr der Verletzung des Persönlichkeitsrechts, zu dem das Recht am eigenen Bild, das Recht auf Privatsphäre, das Recht auf informationelle Selbstbestimmung und das Recht auf persönliche Ehre gehören. Es ist notwendig, durch Regulierung der Menschenfleischsuche ihre gesunde Entwicklung zu garantieren, um möglicherweise die dadurch entstehenden Rechtsverletzungen zu vermeiden. Die Diskussion über Menschenfleischsuche ist auch deswegen wichtig, weil sie ein typisches Beispiel von durch den Internetnutzer begangener Rechtsverletzung zeigt.

Um die Menschenfleischsuche zu regulieren bzw. um die oben genannten Persönlichkeitsrechte zu schützen, ist das chinesische Gesetz von vielen Aspekten unvollständig. Das gleiche Problem existiert auch in Deutschland. Die Rechtsverletzung durch den Internetnutzer und ihre Regulierung stellt für jedes Land neue Probleme dar, die nicht unbedingt durch Gesetzgebung, sondern eher durch Auslegung der vorhandenen Gesetze gelöst werden können. Die Entscheidungspraxis in Deutschland und in China versucht durch Rechtsprechung die Probleme zu lösen.

Zur Regulierung der Menschenfleischsuche soll der Schwerpunkt bei der Regulierung des Verhaltens der Internetnutzer bleiben. Der Veranlasser ist der 
Hauptgrund, warum eine Menschenfleischsuche überhaupt stattfindet. Er soll vor der Einleitung einer Menschenfleischsuche abwägen, ob die Suche wegen öffentlichen Interesses oder anderer Interessen gerechtfertigt ist oder nur zu puren Rechtsverletzungen führen würde. Diese Sorgfaltspflicht des Veranlassers ist für eine gesunde Entwicklung der Menschenfleischsuche nützlich, weil dadurch eine Kontrolle an der Quelle aufgebaut ist. Verletzte er diese Sorgfaltspflicht, muss er für die während der Suche entstehenden Rechtsverletzungen als Anstifter haften.

Der Informationsberichter, der Informationssammler und der Kommentator spielen während der Suche unterschiedliche Rollen. Die Rechtswidrigkeit ihres Verhaltens muss häufig in jedem einzelnen Fall durch eine Interessenabwägung entschieden werden.

Abzuwägen sind auf einer Seite die Persönlichkeitsrechte des Betroffenen und auf der andere Seite die Meinungsfreiheit des Äußernden, das Informationsinteresse der Öffentlichkeit sowie andere gegenüberstehende grundrechtliche Interessen. Für die Interessenabwägung gibt es keine universellen Regeln. Sie muss in jedem einzelnen Fall unter Berücksichtigung der gesamten Konstellation durchgeführt werden. Jedoch ist eine Untersuchung nach ähnlichen Fallgruppen möglich, um einige grundlegende Prinzipien für ähnliche Sachverhalte zu bestimmen.

Die Interessenabwägung ist in Deutschland für die Beurteilung der Persönlichkeitsrechtsverletzung eine allgemein anerkannte Methode. Die Gerichte in China bevorzugen es aber, durch die Subsumtion nach jedem Bestandteil des Eingriffs, nämlich der Tat, der Schuld, des Ergebnisses und der Kausalität zwischen der Tat und dem Ergebnis, eine Persönlichkeitsrechtsverletzung zu beurteilen. Dementsprechend wird die Interessenabwägung durch die engere oder breitere Auslegung der Grenzen der Persönlichkeitsrechte bei der Beurteilung ersetzt.

Nicht alle Menschenfleischsuchen sind von den Internetnutzern eingeleitet. Wegen der zahlreichen Teilnehmer und der damit verbundenen potenziellen wirtschaftlichen Interessen ist die Veranlassung einer Menschenfleischsuche durch den Provider ebenfalls möglich. Im Fall der Rechtsverletzung muss der Provider auch als Anstifter haften.

Bei der Providerhaftung soll zwischen dem ICP und dem ISP unterschieden werden. Der Unterschied liegt darin, dass der ICP die Inhalte auf seiner Webseite entweder selbst editiert oder nach bestimmten Voraussetzungen zu Eigen gemacht. Er hat deswegen für diese Inhalte eine allgemeine Überwachungspflicht und soll für die daraus entstehenden Rechtsverletzungen verantwortlich sein.

In Deutschland haftet der ICP trotz der Anwendung von BDSG nicht anders als ein rechtsverletzender Internetnutzer, außer wenn der ICP als eine „elektronische Presse" angesehen werden könnte, in welchem Fall er aufgrund des 
Medienprivilegs die persönlichen Informationen zum bestimmten Zweck ohne Einwilligung erheben und verwenden kann. Der ICP in China haftet nicht anders als ein rechtsverletzender Internetnutzer.

Im Gegensatz zu dem ICP braucht der ISP prinzipiell nicht für die Inhalte der Menschenfleischsuche verantwortlich sein. Der ISP haftet in Deutschland für die von seinem Nutzer begangener Rechtsverletzung als Störer. Anders als in Deutschland haftet der ISP in China gesamtschuldnerisch mit dem unmittelbaren rechtsverletzenden Internetnutzer. Die gesamtschuldnerische Haftung in China ergibt sich nach der herrschenden Meinung wegen der Mittäterschaft zwischen dem ISP und dem rechtsverletzenden Nutzer. Ein gemeinsamer Vorsatz oder eine gemeinsame Fahrlässigkeit ist für die Mittäterschaft in China nicht notwendig.

Für die Providerhaftung haben Deutschland und China das „Notice-andTake-Down"-Verfahren von den USA übernommen. Deutschland hat darüber hinaus das „Notice-and-Action“-Verfahren für den Beseitigungsanspruch und „Notice-and-keep-off“-Verfahren für den Unterlassungsanspruch entwickelt. Das „Notice-and-Action“-Verfahren bzw. der Kommunikationsprozess ist vielen Kritiken begegnet. Es ist jedoch zurzeit die beste Methode, um die Persönlichkeitsrechte des Betroffenen vor weiteren Rechtsverletzungen im Internet rechtzeitig zu schützen.

Das chinesische Delikthaftungsgesetz hat bei der Übernahme des „Noticeand-Take-Down"-Verfahrens gemäß dem Gesetzestext das "Anti-Notice“Verfahren vernachlässigt. Dies könnte dazu führen, dass die Meinungsfreiheit des Äußernden nicht ausreichend geschützt wird. Ein „Anti-Notice“-Verfahren soll durch Rechtsprechung oder Novellierung des Gesetzes zugefügt werden.

Zur Regulierung der Menschenfleischsuche bzw. zum Schutz des Betroffenen sollen viele Methoden kombiniert verwendet werden. Durch Providerhaftung kann der Betroffene von dem ISP Beseitigung oder Unterlassung verlangen, um die laufende Rechtsverletzung rechtzeitig zu stoppen und die wiederholte Rechtsverletzung zu vermeiden. Im Fall, dass der Provider alle Handlungspflichten geachtet hat, aber das Recht des Betroffene trotzdem verletzt worden ist, soll der Verletzte noch die Möglichkeit haben, direkt Ansprüche gegen den rechtsverletzenden Internetnutzer geltend zu machen.

In Deutschland wird ein allgemeiner Auskunftsanspruch gegen den Provider über den Anmeldedaten der rechtsverletzenden Internetnutzer wegen Persönlichkeitsrechtsverletzung vom BGH abgesagt. Außer dem Fall der Verletzung des Rechts am eigenen Bild kann ein Auskunftsanspruch nur noch verlangt werden, wenn sich das Persönlichkeitsrechtsverletzen, zum Beispiel in Form von Beleidigung oder Verleumdung, zu Straftat wandelt, und der Auskunftsanspruch dann zum Zweck der Strafverfolgung dient. 
Im Vergleich zu Deutschland haben die Gerichte in der Provinz Jiangxi in China eine gute Methode erfunden, in der man nur die IP-Adresse oder ein Pseudonym braucht, um ein Vorverfahren einzuleiten, in dem das Gericht von Amts wegen den rechtsverletzenden Internetnutzer identifizieren kann. Durch diese Methode wird die Aufgabe der Interessenabwägung beim Auskunftsanspruch den Gerichten übertragen, die wegen der Fachkenntnissen besser in der Lage sind, eine richtige Entscheidung zu treffen. Die Methode von der Provinz Jiangxi ist zu empfehlen.

Um den Kommunikationsprozess durchzuführen soll der ISP mindestens die Möglichkeit haben, seine Nutzer zu kontaktieren. Deswegen wird es in Deutschland vorgeschlagen, auf die Anonymität im Internet einigermaßen zu verzichten und stattdessen die Pseudonymität durchzuführen. Der Verzicht der Anonymität soll kombiniert mit der Haftung des ISP für fremde Information betrachtet werden. Es soll eine freie Entscheidung des ISP sein, Anonymität oder Pseudonymität auf seiner Webseite durchzuführen. Wenn er sich für die Anonymität entschieden hat, muss er die fremden Inhalte intensiver prüfen, denn falls er nicht beweisen kann, dass die Inhalte von einem bestimmten Nutzer eingetragen wurden, sollen die Inhalte als von ihm zu Eigen gemacht angesehen werden. Der Provider soll dann als ICP haften. In diesem Fall soll der ISP zwischen der hohen Gefahr der Haftung als ICP und dem potenziellen wirtschaftlichen Interesse wegen der vermehrten Nutzung aufgrund von Anonymität abwägen und frei entscheiden.

Um die Rechtsverletzung und andere Internetgewalt zu vermindern, wurde in China ein Real-Name-System vorgeschlagen. Das Real-Name-System erfordert, dass sich jeder Internetnutzer mit realem Namen und ID-Nummer registrieren muss, bevor er sich im Internet aktiv verhalten darf. Das RNS ist jedoch unverhältnismäßig. Dadurch würden Unsicherheiten der wichtigen persönlichen Daten und eine Beschränkung der Meinungsfreiheit entstehen.

Der Unterschied zwischen dem Real-Name-System in China und der Tendenz zum Verzicht der Anonymität in deutscher Literatur liegt darin, dass das Real-Name-System von der Regierung durchgeführt wird, während der Verzicht der Anonymität eine freie Entscheidung des ISP sein soll. Der Unterschied liegt weiterhin darin, dass der Verzicht der Anonymität Rechtsverletzung-orientiert ist, während Real-Name-System allgemein und umfassend ist. Übrigens gibt es in China noch kein wirkliches Datenschutzgesetz. Im Vergleich mit Deutschland können die persönlichen Daten nicht vor dem Missbrauch des Staats wirksam geschützt werden. Jedoch ist es auch in China zu empfehlen, Pseudonymität für die aktive Teilnahme an der Menschenfleischsuche durchzuführen, um im Fall von Rechtsverletzung die Identifizierung des unmittelbaren Verletzers zu ermöglichen. 
Zur Regulierung der Menschenfleischsuche ist es wichtig, die Rechtsverletzungen von der Quelle an zu vermeiden. Dazu zählen die Erteilung einer hohen Sorgfaltspflicht des Veranlassers über den Verlauf der Suche, ein einfacher Weg zu den Ansprüchen gegen den rechtsverletzenden Internetnutzer und die Pseudonymität für die aktive Teilnahme an einer Menschenfleischsuche.

Außer durch rechtliche Maßnahmen kann die Regulierung der Menschenfleischsuche auch durch die Selbstregulierung durch die Internetnutzer und die Regulierung durch die Provider verwirklicht werden. Es ist auch zu erwarten, dass die Menschenfleischsuche mehr von Journalisten und zuständigen Behörden eingeleitet werden, um die rechtswidrigen Handlungen während der Suche $\mathrm{zu}$ vermeiden.

\section{Die Zukunft der Menschenfleischsuche}

Menschenfleischsuche ist ein Geschöpf der Entwicklung des Internets und ein Phänomen weltweit. Wegen der kulturellen, sozialen und politischen Besonderheiten passiert Menschenfleischsuche in China besonders häufig. Auch heute tauchen im chinesischen Internet ständig neue Fälle von Menschenfleischsuche auf. Es ist zu erwarten, dass Menschenfleischsuche in der Zukunft in China weiter existieren und sich entwickeln wird. Dieser Trend hat bereits die Aufmerksamkeit des chinesischen Gesetzgebers gewonnen. Außer dem Datenschutzgesetz, das schon für mehrere Jahre unter Entwurf ist, hat der Gesetzgeber versucht, Regelungen für den persönlichen Datenschutz durch Änderungen der bestehenden Gesetze im chinesischen Rechtssystem hinzuzufügen. Außerdem hat das Oberste Volksgericht vor kurz „Regelung über einigen Fragen hinsichtlich der anzuwendenden Gesetzen bei der Beurteilung von zivilrechtlichen Streitigkeiten wegen Verletzung der persönlichen Rechte und Interesse durch Internet" als Rechtsprechung erlassen, um genau die Situation der Menschenfleischsuche zu regulieren. Dank der Häufigkeit der Menschenfleischsuche ist China im Bereich von rechtlicher Regulierung der vom Internetnutzer begangenen Persönlichkeitsrechtsverletzung ein Schritt weiter gekommen.

Im Gegensatz zu China wird Menschenfleischsuche in Deutschland nur durch Rechtsprechungen bzw. durch Auslegung der bestehenden Gesetze behandelt, um die alten Gesetze auf die neue Situation anzupassen. Dies gelingt jedoch nur teilweise. Viele relevante Gesetze sind veraltet und können nicht mehr auf die neue Situation angepasst werden. Die Regelungen sind auch nicht konzentriert in einem Gesetz zusammengefasst, sondern in vielen Gesetzen verteilt. Dies führt leicht zu Widrigkeiten zwischen den einzelnen Regelungen. Zum Beispiel, im Fall des Auskunftsanspruchs des Verletzten gegen den Provider auf 
die Anmeldedaten des Verletzers wegen allgemeiner Persönlichkeitsrechtsverletzung hat der BGH zugegeben, dass die Auslegung der bestehenden Gesetze zu einem wenig überzeugenden Ergebnis geführt hat, aber er ohne den Einsatz des Gesetzgebers nichts weiteres machen kann. Es tauchten auch in Deutschland schon Fälle von Menschenfleischsuche auf. Es ist nicht auszuschließen, dass in der Zukunft noch mehr solcher Fälle entstehen werden, weil Menschenfleischsuche nicht nur ein Politik, Sozial oder Kultur relevantes Phänomen ist, sondern auch ein Phänomen mit den technischen und psychologischen Hintergründe in der Internetwelt ist, die für jede Nation gültig sind. Menschenfleischsuche bezogene Rechtsprobleme haben bereits die Aufmerksamkeit der deutschen Juristen gewonnen. Es ist zu erwarten, dass der deutsche Gesetzgeber die bezüglichen Regelungen aktualisiert, um sie der neuen Situation anzupassen.

Der Regulierungsbedarf der Menschenfleischsuche liegt auch darin, dass sie ein typisches Beispiel der durch Internetnutzer begangenen Rechtsverletzung ist. Wegen der Anonymität oder Pseudonymität im Internet hat solche Rechtverletzung eine schwierige Frage an alle Länder gestellt. Es ist weltweit eine Diskussion, durch Providerhaftung oder durch Delikthaftung der unmittelbar rechtsverletzenden Internetnutzer dieses Problem zu lösen.

Der Gesetzgeber in China tendiert zwar dazu, dem Verletzten einen schnellen Zugriff auf den unmittelbar rechtsverletzenden Internetnutzer zu ermöglichen. Gleichzeitig hat er aber fast eine allgemeine Überwachungspflicht dem ISP auferlegt. Statt Störerhaftung muss der ISP in China wegen Pflichtverletzung eine gesamtschuldnerische Haftung mit dem unmittelbaren rechtsverletzenden Internetnutzer tragen. Durch die Absage eines allgemeinen Auskunftsanspruchs auf Meldedaten des Nutzers wegen allgemeiner Persönlichkeitsrechtsverletzung hat Deutschland ebenso eine hohe Pflicht dem ISP gegeben. Jedoch ist die Diskussion in beide Länder nicht zu Ende, sondern steht eher noch am Anfang, weil es von vielen Aspekten aus doch sinnvoller ist, bei der Rechtsverletzung direkt auf den unmittelbaren Verletzer zuzugreifen. Das ist der einzige Weg, die Rechtsverletzungen von der Quelle an zu vermeiden.

Die Antwort, wie man eine Balance zwischen der Providerhaftung und Delikthaftung der Nutzer, zwischen der Anonymität im Internet und dem Persönlichkeitsrechtschutz finden kann, bleibt trotz der Diskussion in dieser Arbeit offen. 


\section{Literaturverzeichnis}

Ahlberg, Hartwig/Götting, Horst-Peter, Beck'scher Online-Kommentar zum Urheberrecht, Stand: 1.10.2014, Edition: 6, München 2014

Amelung, Knut: Die zweite Tagebuchentscheidung des BVerfG, NJW 1990, 1753

Arnsperger, Malte: Feuern und "Motherfucker" brüllen, Stern.de, 13.04.2007, http://www.stern.de/politik/deutschland/bundeswehr-feuern-undmotherfucker-bruellen-586855.html (besucht am 04.04.2015)

Augsburger Allgemeine: Überfall verhindert: Wer ist der unbekannte Helfer? Augsburger Allgemeine, 27.09.2013, http://www.augsburger-allgemeine.de/ bayern/Ueberfall-verhindert-Wer-ist-der-unbekannte-Helfer-id27168107. html (besucht am 04.04.2015)

Bai, Xue: "Ren rou sou suo" zhong de wang luo yin si quan fa lü bao hu yan jiu, Lan zhou xue kan 2010, No. 9, 111

Ballhausen, Miriam/Roggenkamp, Jan Dirk: Personenbezogene Bewertungsplattformen, K\&R 2008, 403

Bamberger, Heinz Georg/Roth, Herbert: Beck'scher Online-Kommentar BGB, Stand: 01.02.2014, Edition: 30, München 2014

Bild: Welpe in Schlucht geworfen: Hunde-Quälerei! US-Soldat aus dem Militär entlassen, http://www.bild.de/news/vermischtes/hund/harte-strafen-fuer-ussoldaten-4821120.bild.html (besucht am 04.04.2015)

Cai, Chang: Wang luo fu wu ti gong zhe qin quan ze ren gui ze de fan si yu chong gou, Studies in Law and Business 2013, No. 2, 113

Chen, Jinchuan: Guan yu wang luo fu wu zhong "bi feng gang” xing zhi de tan tao, Journal of Law Application 2012, No. 9, 25

Chen, Jinchuan: Research about Fault Recognition of ISP, Intellectual Property 2011, No. 2, 56

Chen, Jinchuan: Some Issues in Torts by ISPs, Journal of Law Application 2011, No. 6, 52

Chen, Shuyan: Ge ren xin xi de quan li shu xing ji li fa jia zhi qu xiang de min fa jie xi, Theory Monthly 2011, No. 5, 105

Chen, Xinglan: Ye zhu qian fei guan si bai su wu ye tie chu pan jue shu bei zhi qin fan yin si quan, Chutian Metropolis Daily 30.12.2008, S. 8, http://www.cnhubei. com/ctdsb/ctdsbsgk/ctdsb08/200812/t540763.shtml (besucht am 04.04.2015).

Chen, Zhong: 6 yue xiong ling, 600 ming ren ji ti shi sheng, Da yang wang, 06.06.2005, http://it.sohu.com/20050606/n240093336.shtml (besucht am 04.04.2015) 
Cheng, Yan: The identification of copyright infringement liability of internet service provider, Internet Law Review 2012, No. 1, 201

China Internet Network Information Center (CNNIC): 32th Statistical Report on Internet Development in China, Stand: Juli 2013, http://www.cnnic.cn/hlwfzyj/hlwxzbg/hlwtjbg/201307/t20130717_40664.htm (besucht am 04.04.2015)

China Youth Daily, Mei ti cheng duo bu men jiao cha guan li zhi ge ren xin xi bao hu fa nan chan, China Youth Daily 22.05.2012, http://dailynews.sina. com/gb/chn/chnpolitics/sinacn/20120522/13033411440.html (besucht am 04.04.2015)

Civil Law Department of Legislative Affairs Commission of NPC Standing Committee: Complete works of legislation background and perspective in tort liability law, Peking 2010

Dai, Yingjie: Dao de shen pan zhi yu gong min yin si quan zhi shi, Journal of Zhejiang Institute of Media and Communications 2011, No. 1, 37

Damm, Renate/Rehbock, Klaus: Widerruf, Unterlassung und Schadensersatz in den Medien, 3., neu bearb. Aufl., München 2008

Dammann, Ulrich/Simitis, Spiros: EG-Datenschutzrichtlinie, Kommentar, 1. Aufl., Baden-Baden, 1997.

Däubler, Wolfgang/Klebe, Thomas/Wedde, Peter/Weichert, Thilo: Bundesdatenschutzgesetz, Kompaktkommentar zum BDSG und anderen Gesetzen, 4., vollst. neu bearb. Aufl., Frankfurt am Main 2014

Degenhart, Christoph: Das allgemeine Persönlichkeitsrecht, Art. 2 I i. V. mit Art. 1 I GG, JuS 1992, 364

Deng, Xiaoxia, Wang, Shuhuai: Dui “wang luo bao li” shuo "bu”, Renmin Ribao 10.08.2007

Der Bundesbeauftragte für Datenschutz und Informationsfreiheit (BfDI): Kritik an Vorschlägen zur Öffentlichkeitsfahndung, ZD-Aktuell 2012, 03277

Dieterich, Thomas/Müller-Glöge, Rudi, Erfurter Kommentar zum Arbeitsrecht, 15., neu bearb. Aufl., München 2015

Dix, Alexander: Thesen zum Referat von BlnBDI, Zitat aus: Begleitheft zum Deutschen Juristentag 2012, Thesen der Gutachter \& Regerenten, S. 70 ff, abrufbar unter: http://www.djt.de/fileadmin/downloads/69/120809_djt_69_ thesen_web.pdf. (besucht am 04.04.2015)

Dreier, Horst: Grundgesetz-Kommentar, Bd. 1: Präambel, Artikel 1 - 19, 3. Aufl., Tübingen 2013

Dreier, Thomas/Schulze, Gernot: Urheberrechtsgesetz, Urheberrechtswahrnehmungsgesetz, Kunsturhebergesetz, Kommentar, 4. Aufl., München 2013 
Duchang Unteres Volksgericht, Beschl. v. 22.3.2013 - (2013) du wang chu zi di 2 hao

Ellbogen, Klaus: Die Fluchttagebücher Frank Schmökel und ihre Verwertbarkeit im Strafprozess, NStZ 2001, 460

Ensthaler, Jürgen/Heinemann, Mirko: Die Fortentwicklung der Providerhaftung durch die Rechtsprechung, GRUR 2012, 433

Epping, Volker/Hillgruber, Christian: Beck'scher Online-Kommentar GG, Stand: 1.3.2014, Edition: 20, München 2014

Erbs, Georg/Kohlhaas, Max: Strafrechtliche Nebengesetze, 195. Ergänzungslieferung, München 2013

Erman, Walter: Bürgerliches Gesetzbuch, Bd. 1, 13. neu bearb. Aufl., Köln 2011

Feldmann, Thorsten: Anm. zu BGH, Urt. v. 25.10.2011 - VI ZR 93/10, K\&R 2012, 113

Franz, Stephan: Grundlagen des ökonomischen Ansatzes: das Erklärungskonzept des Homo Oeconomicus, International economics working paper von Universität Potsdam, Potsdam 2004, abrufbar unter http://potsdamer-koepfe. $\mathrm{de} / \mathrm{u} / \mathrm{makrooekonomie/docs/studoc/stud7.pdf.} \mathrm{(besucht} \mathrm{am} \mathrm{04.04.2015)}$

Frenz, Walter: Berufsbezogene Vorwürfe und Medienveröffentlichungen, ZUM 2012, 282

Frenz, Walter: Konkretisierte Abwägung zwischen Pressefreiheit und Persönlichkeitsschutz, NJW 2012, 1039

Fromm, Friedrich Karl/Nordemann, Wilhelm: Urheberrecht: Kommentar zum Urheberrechtsgesetz, Verlagsgesetz, Urheberrechtswahrnehmungsgesetz, 10., überarb. und erg. Aufl., Stuttgart 2008

Gaier, Reinhard: Münchener Kommentar zum Bürgerlichen Gesetzbuch, Band 6, 6. Aufl, München 2013

Gao, Wenmiao: Fa zhi shi ye xia de wang luo shi ming zhi tan xi, Lanzhou Academic Journal 2012, No. 3, 167

Gola, Peter/Schomerus, Rudolf: Bundesdatenschutzgesetz, Kommentar, 11. Aufl., München 2012

Gounalakis, Georgios/Klein, Catherine: Zulässigkeit von personenbezogenen Bewertungsplattformen - Die „Spickmich”-Entscheidung des BGH vom 23. 6. 2009, NJW 2010, 566

Gounalakis, Georgios/Rhode, Lars: Persönlichkeitsschutz im Internet: Grundlagen und Online-Spezifika, München 2002

Gräbig, Johannes: Aktuelle Entwicklungen bei Haftung für mittelbare Rechtsverletzungen, Vom Störer zum Täter - ein neues einheitliches Haftungskonzept? MMR 2011, 504 
Greve, Holger/Schärdel, Florian: Der digitale Pranger - Bewertungsportale im Internet, MMR 2008, 644

Guo, Hongpeng/Huang, Hui: Quan guo shou ge zhen dui wang luo qin quan she song wang tie su qian jin ling fa chu, Legal Daily, 25.03.2013, http:// www.legaldaily.com.cn/index_article/content/2013-03/25/content_4308890. htm?node $=5954$ (besucht am 04.04.2015)

Habersack, Mathias: Münchener Kommentar zum Bürgerlichen Gesetzbuch, Band 5, 6. Aufl, München 2013

Hahn, Werner/Vesting, Thomas, Beck'scher Kommentar zum Rundfunkrecht, 3. Aufl., München 2012

Han, Gong: Die Auslegung und Anwendung der "Antworten zu einigen Fragen hinsichtlich der Beurteilung der Fälle über Ehrenverletzung" des Obersten Volksgerichthofs, http://www.npc.gov.cn/huiyi/lfzt/qqzrfca/2008-12/21/content_1462861.htm (besucht am 04.04.2015).

Han, Ning: A Probe into the Legality of Real-Name Registration on the Weibo - From a Perspective of Freedom of Speech, Legal Science Monthly 2012, No. 4,3

Härting, Niko: "Prangerwirkung” und “Zeitfaktor": 14 Thesen zu Meinungsfreiheit, Persönlichkeitsrechten und Datenschutz im Netz, CR 2009, 21

He, Yucong: Yun nan shi lin jing qu dao you bei bao yu you ke dui ma, guan fang zheng diao cha, Kun ming xin xi gang 07.01.2014, http://news.qq.com/a/ 20140107/013683.htm (besucht am 04.04.2015)

Heckmann, Dirk: Persönlichkeitsschutz im Internet: Anonymität der IT-Nutzung und permanente Datenverknüpfung als Herausforderungen für Ehrschutz und Profilschutz, NJW 2012, 2631

Herwig, Stefan: Austarierung von Anonymität und Verantwortung im Netz - Ein kommunikationswissenschaftlicher Zwischenruf, ZD 2012, 558

Hessenschau: Sextäter wird auf Facebook gejagt, Hessenschau 05.11.2013, http:// www.hr-online.de/website/rubriken/nachrichten/indexhessen 34938. jsp? rubrik=36082\&xtmc=Facebook-Jagd\%20auf\%20Sex-T\%FDter\&type $=$ d\&xtcr=1\&key=standard_document_50022359 (besucht am 04.04.2015)

Hoeren, Thomas: Anm. zu BGH, Urt. v. 25.10.2011 - VI ZR 93/10, MMR 2012, 124

Hou, Li/Wang, Xiaomi: Gong min xiao xiang quan ruo gan wen ti de zai si kao, Legal Science Monthly 1992, No. 2, 15

$\mathrm{Hu}, \mathrm{Dan}$ : The conflict and balance between public figures to privacy and freedom of the press, unveröffentlichte Magisterarbeit von Hunan Universtität, Changsha 2012 
Hu, Qingfang: Bao lu kuang shi dai, Du shu 2008, No. 11, 148

Huang, Liangyou: On the Confirmation of the Litigant in Internet Tort Action, Hebei Law Science 2012, No. 10, 75

Huang, Ping: Views on Protection of Personal Information from the First Case of Human Flesh Search Engine, Journal of Shanghai Institute of Political Science \& Law 2009, No. 2, 16

Jauernig, Othmar/Stürner, Rolf/Berger, Christian, Bürgerliches Gesetzbuch Kommentar, 15., neubearb. Aufl., München 2014

Jiang, Zengpei: Wang luo cheng wei fan fu bai de li jian, Dong fang wang, http:// www.jxnews.com.cn/jxcomment/system/2008/01/02/002647086.shtml (besucht am 04.04.2015)

Jing, Huibao/Chang, Xiujiao: Definition of Private Information, Journal of Law Application 2011, No. 3, 90

Jürgens, Uwe: Anm. zu BGH, Urt. v. 19. 4. 2007 - I ZR 35/04, K\&R 2007, 392

Karg, Moritz: IP-Adressen sind personenbezogene Verkehrsdaten, MMRAktuell 2011, 315811

Klein, Susanne: Auskunftsanspruch bei Persönlichkeitsrechtsverletzungen im Internet: Rechtlich unmöglich und/oder politisch nicht gewollt? GRUR-Prax 2014, 539

Kong, Xiangjun: Discussions on Internet Dissemination Acts, People's Judicature (application) 2012, No. 7, 59

Ladeur, Karl-Heinz/Gostomzyk, Tobias: Der Schutz von Persönlichkeitsrechten gegen Meinungsäußerungen in Blogs: Geht die große Zeit des privaten Presserechts im Internet zu Ende? NJW 2012, 710

Leible, Stefan/Sosnitza, Olaf: "3 - 2 - 1 - meins!" und das TDG: Zur Haftung von Internetauktionshäusern für rechtswidrige Inhalte, WRP 2004, 592

Leonard, Tom: Love at first site on the New York subway, 10.11.2007, http:// www.telegraph.co.uk/news/uknews/1568941/Love-at-first-site-on-the-NewYork-subway.html (besucht am 04.04.2015)

Ler/dpa/ddp: Bundeswehr: Erste Geständnisse im Skandal um TotenschädelFotos, Spiegel online, 31.10.2006, http://www.spiegel.de/politik/deutschland/ bundeswehr-erste-gestaendnisse-im-skandal-um-totenschaedel-fotosa-445561.html (besucht am 04.04.2015)

Li, Fang: "Ren rou sou suo", tian tang vs di yu? Shi chang bao, 17.06.2008, http:// news.xinhuanet.com/focus/2008-06/17/content_8384135.htm (besucht am 04.04.2015)

Li, Guoying: The Explaining Capabilities of Information Rights are the Legal Analysis Tool of "Human Flesh Search", Jinling Law Review 2010, No. 1, 70 
Li, Jun/Zhang, Jun: Legal Analysis on Infringement Cases Concerning Internet Mass Hunting, People's Judicature (case) 2008, No. 22, 24

Li, Xiufen: Privacy: The Scope of Protection, Contemporary Law Review 2004, 18 No. 4,98

Li, Yifei: Tort liability of privacy and legal regulation causing by "Human-flesh Searching", Journal of Northwest University (Philosophy and Social Sciences Edition) 2010, No. 5, 67

Li, Yougen: Publicizing the Judicial Judgment and Protection of Privacy, Legal Science Monthly 2010, No. 5, 126

Lian, Xiao: View from the Civil Law Perspective Research about the Institutional Arrangements of the Protection of Privacy and Personal Pnformation, Journal of Heilongjiang Administrative Cadre Institute of Politics and Law 2010, No. 8, 58

Liang, Chen: The Social Mechanism and Business Model of Manpower Search, Internet Law Watch 2008, No. 1, 7

Liang, Huixing/Liao, Xinzhong: Yin si de ben zhi yu yin si quan de gai nian (The essence of privacy and the concept of the right to privacy), People's Judicature 2003, No. 4, 42

Liang, Zhiwen: Wo guo fa shang de bi feng gang gui ze: li yi shi heng yu li fa wan shan, Electronics Intellectual Property 2011, No. 9, 33

Liu, Deliang: The Theory and Practice of Civil and Commercial Law in the Internet Age, Peking 2008

Liu, Haitao/Zheng, Jinxiong/Shen, Rong: Zhong guo xin wen guan si er shi nian: 1987-2007, Peking 2007

Liu, Han: Privacy, Free Speech, and the Chinese Internet Culture: the Predicament of Human-Flesh Search Engine Regulation, Peking University Law Journal 2011, 870

Liu, Peihe/Tian, Yining: The First Case of Human Flesh Search: Analysis in Legal Theory, Contemporary Law Review 2009, No. 3, 127

Liu, Wenjie: Security Duty of Internet Service Providers, Peking University Law Journal 2012, No. 2, 395

Liu, Xiaochun: Reflection and reconstruction of ISP's tort liability: take video share sites as example, Internet Law Review 2011, No. 1, 3

Liu, Yijun/Liu, Haidong: Law Application on Violation of Privacy and Right of Fame by Manpower Search, Journal of Law Application 2010, No. 7, 81

Loewenheim, Ulrich: Handbuch des Urheberrechts, 2. Aufl., Frankfurt am Main 2010 
Lu, Chunya: On the Tort Liability of Network Service Provider, Journal of Henan University of Economics and Law 2012, No. 5, 58

Lu, Donghong: Zhang tie pan jue shu bu gou cheng qin quan, Southern Metropolis Daily, 1.10.2002

Lu, Wei: Biao da zi you quan yu wang luo shi ming zhi de fa lü bo yi, Lanzhou Academic Journal 2012, No. 9, 161

Lu, Yixiao: On Right of Claim for Infringement of Personality Rights in Human Flesh Search, Journal of Ningbo Radio \& TV Universtity 2009, No. 4, 4

Luo, Kun: Dao de, fa lü yu "ren rou sou suo" zhong de yin si quan, People’s Court Daily, 12.08.2008

Ma, Xudong: Cong "ren rou sou suo" kan wang luo zhuang tai xia de yan lun biao da zi you, Journal of Tianzhong 2011, No. 1, 38

Magnie, Mark: Cyberspace gumshoes are afoot, Los Angeles Times, 23.11.2008, http://articles.latimes.com/2008/nov/23/world/fg-scandal23 (besucht am 04.04.2015)

Maunz, Theodor/Dürig, Günter: Grundgesetz-Kommentar, Band I, 69. Ergänzungslieferung, München 2013

Mayer-Schönberger, Viktor: Delete: The Virtue of Forgetting in the Digital Age, Princeton 2009

Mei, Shaozu: The basic problems of the protection of personal information, Academic Journal of Suzhou University (Philosophy and Social Science) 2005, No. 2, 25

Mei, Xiaying/Liu, Ming: The Present Confinement and Value Judgement of Internet Tort Liability: Focusing on Art. 36 of China’s Tort Liability Law, Science of Law 2013, No. 2, 82

Meng chi xue gao: Shan yong wang luo li jian - ren rou sou suo, http://dzh.mop. com/whbm/20080828/0/F53OSOI1b228c4z8.shtml (besucht am 04.04.2015)

Moos, Flemming: Unzulässiger Handel mit Persönlichkeitsprofilen? - Erstellung und Vermarktung kommerzieller Datenbanken mit Personenbezug, MMR 2006, 718

Müller-Broich, Jan D.: Telemediengesetz, 1. Aufl., Baden-Baden 2012

Neunhoeffer, Friederike, Das Presseprivileg im Datenschutzrecht: eine rechtsvergleichende Betrachtung des deutschen und des englischen Rechts, Tübingen 2005

Nieland, Holger: Störerhaftung bei Meinungsforen im Internet - Nachträgliche Löschungspflicht oder Pflicht zur Eingangskontrolle? NJW 2010, 1494

Oberwetter, Christian: Bewerberprofilerstellung durch das Internet - Verstoß gegen das Datenschutzrecht? BB 2008, 1562 
Ostendorf, Heribert/Frahm, Lorenz Nicolai/Doege, Felix: Internetaufrufe zur Lynchjustiz und organisiertes Mobbing, NStZ 2012, 529

Palandt, Otto: Bürgerliches Gesetzbuch, mit Nebengesetzen, 74., neu bearb. Aufl., München 2015

Peifer, Karl-Nikolaus/Kamp, Johannes: Datenschutz und Persönlichkeitsrecht - Anwendung der Grundsätze über Produktkritik auf das Bewertungsportal "spickmich.de"? ZUM 2009, 185

Peng, Juan: The Definition of Violating the Portrait Right According to Law Interpretation, Journal of Political Science and Law 2004, No. 3, 23

Peng, Xinghong: Affirmation of subjective faults of internet service providers, Journal of Hebei North University (Social Science Edition) 2013, No. 2, 76

Phsc/jaar: Schon 100 Hinweise auf Sexualstraftäter, hr-online.de, 30.10.2013, http://www.hr-online.de/website/rubriken/nachrichten/indexhessen34938. jsp?rubrik=36082\&key=standard_document_49977267 (besucht am 04.04.2015)

Qi, Aimin: Saving the personality from the information society: general introduction to personal information protection law, Peking 2009

Qi, Aimin: Yi wan bei de li fa tui jin ge ren xin xi bao hu, Chinese Social Sciences Today, 02.04.2012, S. A07

Qi, Le: At the Vision of the Rule of Law Under the Norms of "Human Flesh Search", Journal of Inner Mongolia University for Nationalities (Social Sciences) 2009, No. 3, 104

Qian, Yuwen/Zhang, Jialin: On the Liability of Internet Service Provider's Trademark Infringement, Intellectual Property 2013, No. 2, 44

Qin, Qianhong/Li, Gaoya: Gong min yi si quan bao hu de fan si yu chong gou - yi "ren rou sou suo" wei shi jiao, Study and Practice 2011, No. 11, 57

Rauscher, Thomas/Wax, Peter/Wenzel, Joachim: Münchener Kommentar zur Zivilprozessordnung mit Gerichtsverfassungsgesetz und Nebengesetzen, Band. 1, 4. Aufl., München 2013

Rempe, Christoph: Anm. zu OLG Hamburg, Beschl. v. 13.5.2013 - 5 W 41/13, MMR 2013, 533, 534

Roggenkamp, Jan Dirk: Unanwendbarkeit des Haftungsprivilegs nach TMG auf vorbeugenden Unterlassungsanspruch, Anm. zu BGH, Urt. v. 19.4.2007 - I ZR 35/04 - Internetversteigerung II, jurisPR-ITR 11/2007 Anm. 2.

Rössel, Markus: Filterpflichten des Providers im Lichte des EuGH, CR 2011, 589

Rühl, Giesela: Anm. zu BGH, Urt. v. 25.10.2011 - VI ZR 93/10, LMK 2012, 338417

Säcker, Franz Jürgen/Rixecker, Roland: Münchener Kommentar zum Bürgerlichen Gesetzbuch, Band 1, 1. Halbband, 6. Aufl, München 2012 
Saenger, Ingo: Zivilprozessordnung, FamFG, Europäisches Verfahrensrecht, Handkommentar, 5. Aufl., Baden-Baden 2013

Sajuntz, Sascha: Die Entwicklung des Presse- und Äußerungsrechts in den Jahren 2012/2013, NJW 2014, 25

Schnabel, Christoph: Das Recht am eigenen Bild und der Datenschutz: Die richterrechtliche Dogmatik zur Einwilligung vor dem Hintergrund europarechtlicher Einflüsse des Datenschutzes, ZUM 2008, 657

Schönke, Adolf/Schröder, Horst: Strafgesetzbuch, Kommentar, 28. neu bearb. Aufl., München 2010

Seidel, Janine/Nink, Judith: Personensuchmaschinen: Rechtliche Fragestellungen, CR 2009, 666

Shi, Bifan: Empirical Analyses of Human-powered Search,Faye Wong Case and Privacy Protection, Zhejiang Social Sciences 2011, No. 8, 34

Shi, Jiayou: The protection of personal information under the circumstance of internet, Journal of the University of Suzhou 2012, No.6, 85

Shi, Shangkuan, Zhai fa zong lun, Peking 2000

Shi, Xueqing/Wang, Yong: "Cape of Storms" or "Safe Harbor", Intellectual Property 2009, No. 2, 23

Simitis, Spiros: Bundesdatenschutzgesetz, 8., neu bearb. Aufl., Baden-Baden 2014

Song, Wei/Tan, Jing/Guo, Peng: Zui gao jian cheng fan fu bai li bu kai min zhong ju bao, ju bao fa ji dai chu tai, Zheng yi wang, 02.07.2008, http://news.xinhuanet.com/legal/2008-07/02/content_8473380.htm (besucht am 04.04.2015)

Sou hu she qu bian ji bu: Che zhan zui mei qing jie gong MM shen fen bao guang, http://hot.club.sohu.com/s2008/chezhanzuimeiqingjiegong/(besucht am 04.04.2015)

Spindler, Gerald/Schuster, Fabian: Recht der elektronischen Medien, Kommentar, 2. Aufl., München 2011

Spindler, Gerald: Anm. zu BGH, Urt. v. 19.4.2007 - I ZR 35/04 - Internetversteigerung II, MMR 2007, 511

Spindler, Gerald: Datenschutz- und Persönlichkeitsrechte im Internet - der Rahmen für Forschungsaufgaben und Reformbedarf, GRUR 2013, 996

Spindler, Gerald: Persönlichkeitsrecht und Datenschutz im Internet - Anforderungen und Grenzen einer Regulierung, NJW-Beil. 2012, 98

Spindler, Gerald: Störerhaftung des Host-Providers bei Persönlichkeitsrechtsverletzungen, CR 2012, 176 
Stern.de: Neue Skandalfotos aufgetaucht, Stern.de, 26.10.2006, http://www.stern. de/politik/deutschland/afghanistan-neue-skandalfotos-aufgetaucht-574968. html (besucht am 04.04.2015)

$\mathrm{Su}$, Zhe: The Legal Regulation on Human-flesh Searching, Hebei Law Science 2012, No. 2, 26

Sun, Xiaolin: Research on the General Network Infringement Case Method, Science-Technology and Law 2012, No. 3, 84

Thomas, Heinz/Putzo, Hans: Zivilprozessordnung-Kommentar, 34. Aufl., München 2013

Tian, Feilong: Manpower Search: Internet Public Space, Social Function and Law Regulation, Internet Law Review 2009, 80

Tismer, Silvana: Leinefelde: Unfallopfer durch Engagement und Internet identifiziert, TLZ, 26.11.2013, http://eichsfeld.tlz.de/web/lokal/leben/blaulicht/ detail/-/specific/Leinefelde-Unfallopfer-durch-Engagement-und-Internetidentifiziert-577877368 (besucht am 04.04.2015)

Topic Research Group of No.3 Criminal Division: Research on the Crime of Defamation through Internet, People's Judicature Appliction 2012, No. 21, 17

Uffeln, Malte Jörg/Günther, Lucas: Rechtliche Probleme bei der Nutzung sozialer Netzwerke - wer-kennt-wen, StudiVZ und Co., unveröffentlichter Aufsatz, online abrufbar unter "Arbeitshilfen für Vereine" von http://www.kanzleiuffeln.de/ku/(besucht am 04.04.2015)

Ulmer, Peter/Rebmann, Kurt, Münchener Kommentar zum Bürgerlichen Gesetzbuch, 3. Aufl., München 1997

Vorwerk, Volker/Wolf, Christian: Beck'scher Online-Kommentar ZPO, Stand: 15.03.2014, Edition: 12, München 2014

Volkmann, Christian: Aktuelle Entwicklungen in der Providerhaftung im Jahr 2011, K\&R 2012, 381

von Staudinger, Julius: Kommentar zum Bürgerlichen Gesetzbuch mit Einführungsgesetz und Nebengesetzen, Buch 2: $\$ \$ 823$ - 825, 13. bearb., Berlin 1999

von Staudinger, Julius: Kommentar zum Bürgerlichen Gesetzbuch mit Einführungsgesetz und Nebengesetzen, Eckpfeiler des Zivilrechts, neu bearb., Berlin 2012

von Staudinger, Julius: Kommentar zum Bürgerlichen Gesetzbuch mit Einführungsgesetz und Nebengesetzen, Eigentum 3, neu bearb., Berlin 2013

von Staudinger, Julius: Kommentar zum Bürgerlichen Gesetzbuch mit Einführungsgesetz und Nebengesetzen, Einleitung zum BGB und Allgemeiner Teil 1, neu bearb., Berlin 2013 
von Staudinger, Julius: Kommentar zum Bürgerlichen Gesetzbuch mit Einführungsgesetz und Nebengesetzen, Schadensersatzrecht, neu bearb., Berlin 2005

Wanckel, Endress: Personenbezogene Prominentenberichterstattung anlässlich zeitgeschichtlicher Ereignisse, NJW 2011, 726

Wandtke, Artur-Axel/Bullinger, Winfried: Praxiskommentar zum Urheberrecht, 3., neu bearb. Aufl., München 2009

Wang, Chengwei: Social Construction of Policy Veto: Several Cases on the Failures of Legislation to Prohibit "Human Flesh Search" in China, Journal of Public Management 2011, No. 4, 21

Wang, Gang: Wei bo shi ming zhi bei hou de guo zheng tong, The Time Weekly, 09.01.2012

Wang, Liming/Ge, Weibao: Law forefront dialogue - Personality rights and tort law studies, Peking 2006

Wang, Liming/Yang, Lixin: Ren ge quan yu xin wen qin quan, Peking 1995

Wang, Liming: The Development and Improvement of Personality Law: from the Perspective of the Protection of Human Dignity, Science of Law 2013, No. 4,166

Wang, Liming: The Position of the System of the Right of Personality in Chinese Civil Code, Chinese Journal of Law 2003, No. 2, 32

Wang, Liming, Qin quan xing wei fa yan jiu (Shang juan), Peking 2004.

Wang, Qian: Shi pin fen xiang wang zhan zhu zuo quan qin quan wen ti yan jiu, Studies in Law and Business 2008, No. 4, 42

Wang, Wei/Wang, Luyao: Nü zi yin wei gei yun fu rang zuo bei ren rou sou suo yin fa zheng yi, Chongqing shang bao, 17.08.2012, http://news.sina.com. $\mathrm{cn} / \mathrm{s} / 2012-08-17 / 152924987638 . s h t m l$ (besucht am 04.04.2015)

Wang, Xiuzhe: On the constitutional protection of the right of privacy, Peking 2007

Wang, Yijun: "Ren rou sou suo" tian shi yu mo gui de yong bao, China Youth Daily, 24.9.2008, http://www.cq.xinhuanet.com/2008-09/24/content_14479879. htm (besucht am 04.04.2015)

Wang, Zejian, Qin quan xing wei fa (Di 2 ce), Taibei 2006

Wang, Zhigang: On "Right of Anonymous Speech Online" in American Law, Journal of Beijing University of Aeronautics and Astronautics (Social Sciences Edition) 2006, No. 6, 46

Warren, Samuel/Brandeis, Louis: The Right to Privacy, Harvard Law Review 1890, Vol. IV No. 5, 193

Weichert, Thilo: BDSG-Novelle zum Schutz von Internet-Inhaltsdaten, DuD 2009, 7 
Weichert, Thilo: Datenschutz bei Internetveröffentlichungen, VuR 2009, 323

Weng, Guomin/Wang, Chenghong: The conflict of the right to privacy and the right to know, Journal of Zhejiang Universtity (Humanities and Social Sciences) 2002, No. 2, 33

Wiegand, Ralf: Protest gegen jugendlichen Sexualstraftäter: Menschenauflauf fordert Todesstrafe, sueddeutsche.de, 07.03.2012, http://www.sueddeutsche. de/leben/protest-gegen-jugendlichen-sexualstraftaeter-menschenauflauffordert-todesstrafe-1.1302098 (besucht am 04.04.2015)

Wilmer, Thomas: Überspannte Prüfpflichten für Host-Provider? Vorschlag für eine Haftungsmatrix, NJW 2008, 1845

Wölfl, Bernd: Sphärentheorie und Vorbehalt des Gesetzes, NVwZ 2002, 49

Wotu: Facebook-Jagd auf Sex-Täter, hr-online.de, 06.11.2013, http://www.hronline.de/website/rubriken/nachrichten/indexhessen34938.jsp? rubrik= 36082\&key=standard_document_50022359 (besucht am 04.04.2015)

$\mathrm{Wu}, \mathrm{Bo}$ : Research of the illegality of "cyber manhunt", unveröffentlichte Magisterarbeit von Chinesischer Universität für Politikwissenschaft und Recht, Peking 2011

Wu, Haijie: Shou li QQ xiang yue zi sha an er shen, fa yuan bo hui si zhe jia shu dui teng xun gong si de sun hai pei chang qing qiu, Youth Times 11.02.2012, S. A04

$\mathrm{Wu}$, Handong: Qin quan ze ren fa shi ye xia de wang luo qin quan ze ren jie xi, Studies in Law and Business 2010, No. 6, 29

$\mathrm{Wu}$, Handong: Tort Liability for Indirect Infringement of Copyright in the Internet according to Article 36 of the Tort Law PRC, China Legal Science 2011, No. 2, 38

Wu, Zheng: Nüe you quan shi pin bao guang, zhu yi mei jun ji min fen, Xin hua wang 06.03.2008, http://news.xinhuanet.com/newscenter/2008-03/06/content_7720461.htm (besucht am 04.04.2015)

Xiang, Pin: Rights conflicting and balaced on cyber manhunt, The Rule of Law Forum 2011, No. 4, 86

Xiao, Han: Gong gong ren wu: wu yin hu er? South Reviews 2003, No. 4, 32

Xiao, Qunzhong: On the ancient Chinese tradition of neighborhood relationships and moral regulation, Confucius Studies 2009, No. 4, 17

Xiao, Rong: The Position of Cyber Speech in an Even Realization of Citizen's Basic Rights, Legal Science Monthly 2012, No. 5, 75

Xie, Hongfei: Yan lun zi you yu quan li bao hu de jian nan tiao he - "qin quan ze ren fa" zhong wang luo qin quan gui ze zhi jie du, Procuratorial View 2010, No. 3,26 
Xie, Xuekai: Third-party Liability of the Internet Service Provider: Theories and Legislations Revisited, Oriental Law 2013, No. 2, 149

Xiong, Jingbo: A Solution to Conflict Between Right of Reputation and Freedom of Expression, Ecupl Journal 2011, No. 6, 28

$\mathrm{Xu}$, Wei: The Redefinition and Systematic Influence of Notice and Takedown Regime, Modern Law Science 2013, No. 1, 58

$\mathrm{Xu}$, Yuxiang/Pan, Ying: Wang luo fa bu pan jue shu bu qin fan yin si, Jiangsu Legal News, 21.1.2010, S. 1, http://jsfzb.xhby.net/html/2010-01/21/content_96900.htm (besucht am 04.04.2015)

$\mathrm{Xu}$, Zhenzeng: On the Network Real-name System in the View of Democratic Politics, Hebei Law Science 2012, No. 9, 166

Xue, Hong: Wang luo fu wu ti gong zhe zhong jie ze ren gou cheng de bi jiao yan jiu, China Copyright 2011, No. 5, 36

Xue, Xia: Sociological Reflection about "human-powered Search"Phenomenon, China Youth Study 2009, No. 1, 92

Yang, Fuzhong: Constitutional Protection on Citizen's Anonymous Expression Rights in Networks: Also on the Legitimacy of the Real Name System in Networks, Studies in Law and Business 2012, No. 5, 32

Yang, Jianlin: Zhang tie pan jue shu yin fa ming yu quan guan si, fa yuan ren ding bu gou cheng qin quan, Nan guo jin bao, 10.8.2005, http://www.zhengyanli. org/newshow.asp?id=1573 (besucht am 04.04.2015)

Yang, Lixin/Li, Jialun: The Anti-notification in Internet Tort Liability and Its Effect, Science of Law 2012, No. 2, 157

Yang, Lixin: A propositional version with explaination for tort liability law draft of China, 1. Aufl., Peking 2007

Yang, Lixin: Comprehension and Interpretation of Internet Infringement Liability Regulated in the Tort Law, Journal of National Prosecutors College 2010, No. 2, 3

Yang, Lixin: Jie jue "ren rou sou suo" zhong de wei fa xing wei guan jian zai yu yi fa gui fan wang luo xing wei, Netinfo Security 2008, No. 10, 7

Yang, Lixin: Monograph on personal right, 1. Aufl., Peking 2005

Yang, Lixin, Qin quan fa lun, 3. Aufl., Peking 2005

Yang, Lixin: Ren shen quan fa lun, 3. Aufl., Peking 2006

Yang, Lixin: Tort Liability Law, 2. Aufl., Peking 2012

Yang, Qinghui: Xin lang bo ke chu xian bu dang yan lun, shou hai fang su qin quan wei huo zhi chi, Chinacourt.org, 20.02.2010, http://old.chinacourt.org/ html/article/201001/20/392004.shtml/2011-08-04 (besucht am 04.04.2015) 
Yao, Hong: Zhong hua ren min gong he guo min shi su song fa shi yi, Peking 2007

Yao, Yuerong: On the information on the right to self-determination as a fundamental right in our justification, Political Science and Law 2012, No. 4, 72

Yao, Yuerong: The Protection of Personal Information in the View of Constitution, unveröffentlichte Doktorarbeit von East China University of Political Science and Law, Shanghai 2011

Yin, Yijun: Personality right protection of the involuntary public figure, unveröffentlichte Magisterarbeit von Yantai Universtität, Yantai 2012

Yu, Xiaohai: "Human Flesh Search": the Distinction and the Dual Perspective from Facts and Norms, Journal of Dezhou University 2009, No. 3, 90

Yuan, Xueshi: Improvement on Liability Rule for Infringement in Perspective of Alluring Photo Incident, Political Science and Law 2008, No. 4, 19

Zhai, Xiangjuan: Legal Regulation of Internet Violence - Some Thought Provoked by "Human Flesh Search" Engines, Journal of North China Institute of Water Conservancy and Hydroelectric Power (Social Science) 2010, No. 5,128

Zhang, Bing: Oppugning the Legitimacy of the Criminal Regulation of Human Flesh Search, Journal of Heilongiiang Administrative Cadre Institute of Politics And Law 2010, No. 1, 51

Zhang, Di: Legal protection for the right of privacy beneath freedom of the press, unveröffentlichte Magisterarbeit von Jilin Universtität, Changchun 2011

Zhang, Jianwen: The Expansion of Privacy Protection and the Development of the Protection Way in China from "Faye Wang Case", Journal of Henan University of Economics and Law 2012, No. 2, 95

Zhang, Shujun/Du, Jin: The transfer, difficulties and solutions of Human Search: deal with the investigation rights, reputation rights and privacy rights, Journal of Yunnan University Law Edition 2009, No. 4, 66

Zhang, Wei, Analysis and Application of Liabilities without Fault for Vehicle Accidents, Law Science Magazine 2006, No. 3, 91.

Zhang, Xinbao: Hu lian wang shang de qin quan wen ti yan jiu, 1. Aufl., Peking 2003

Zhang, Xinbao: Legal protection of the right to privacy, 2. Aufl., Peking 2004

Zhang, Xuejun/Wang, Fei/Guo, Zhixia: 600 ming ren shou ji hao bei wang zhan bao guang, zhong ming xing bu kan sao rao, Bei jing yu le xin bao, 05.06.2005, http://news.xinhuanet.com/newmedia/2005-06/05/content_3045908.htm (besucht am 04.04.2015) 
Zhang, Zhuochen: "Ren rou sou suo" zhi fa li tan jiu, Science \& Technology Information 2009, No. 11, 770

Zhang, Zicheng: Talking about "Human Flesh Search" in the Dimension of Constitution - Starting from related articles of Xuzhou for Safety Protection of Computer Information Systems, Academic Exchange 2009, No. 4, 62

Zhang, Zuoguo: Thinking of Reasonable Limitation of Freedom of Speech on the Internet from "Human Flesh Search", Western Law Review 2009, No. 6, 7

Zhao, Qi: The Protection of Personal Information in Judgments Online, Legal Forum 2012, No. 6, 115

Zheng, Gencheng: The Ethical Consideration on "Internet Man-hunting”, Morality and Civilization 2010, No. 5, 121

Zheng, Renrong: Analysis of British ISP Liability of another Publication and Revelation to China, Public Administration \& Law 2011, No. 12, 108

Zhou, Bo/Yang, Kangrui: Research on Joint Liability of Internet Service Provider: Based on View of Tort of Internet Copy Rights, Chinese Journal of Law 2012, No. 1, 108

Zhou, Hua: Lun Wang luo fu wu ti gong zhe de ze ren xian ding, Journal of China Three Gorges University (Humanities \& Social Sciences) 2012, No. 5, 87

Zhou, Xiaochen: Cong "Wang Fei an" tan "ren rou sou suo" suo she ji de qin quan fa wen ti, Netinfo Security 2008, No. 10, 13

Zhou, Yongkun: Wang luo shi ming zhi li fa ping xi, Jinan Journal (Philosophy and Social Sciences) 2013, No. 2, 1

Zhou, Youjun: Lun "ren rou sou suo" zhong de yin si quan bao hu (Privacy Protection in Human Flesh Search Engine), Netinfo Security 2009, No. 2, 49

Zhou, Yuanqing: Wei bo xian xiang de fa lü tou shi - yi yin si quan wei shi jiao, Law Science Magazine 2011, No. S1, 199

Zhu, Juan: On Human Flesh Search Engine as a Spontaneous Order - From the Perspective of Hayek's Doctrine of Dualistic Social Order, Science of Law 2009, No. 1, 47

Zhu, Wei: Su qian jin ling bu shi yong wang luo min yu qin quan, Legal Evening News, 10.05.2013, http://www.zxxk.com/article/245604.html (besucht am 04.04.2015) 
